\title{
An In Vivo Evaluation of Pulmonary Immune Response and Toxicity Following Combined Respiratory Exposure to Crystalline Silica and Diesel Particulate Matter
}

Breanne Yingling Farris

Follow this and additional works at: https://researchrepository.wvu.edu/etd

\section{Recommended Citation}

Farris, Breanne Yingling, "An In Vivo Evaluation of Pulmonary Immune Response and Toxicity Following Combined Respiratory Exposure to Crystalline Silica and Diesel Particulate Matter" (2017). Graduate Theses, Dissertations, and Problem Reports. 5580.

https://researchrepository.wvu.edu/etd/5580

This Dissertation is protected by copyright and/or related rights. It has been brought to you by the The Research Repository @ WVU with permission from the rights-holder(s). You are free to use this Dissertation in any way that is permitted by the copyright and related rights legislation that applies to your use. For other uses you must obtain permission from the rights-holder(s) directly, unless additional rights are indicated by a Creative Commons license in the record and/ or on the work itself. This Dissertation has been accepted for inclusion in WVU Graduate Theses, Dissertations, and Problem Reports collection by an authorized administrator of The Research Repository @ WVU.

For more information, please contact researchrepository@mail.wvu.edu. 
An in vivo evaluation of pulmonary immune response and toxicity following combined respiratory exposure to crystalline silica and diesel particulate matter

\title{
Breanne Yingling Farris
}

\author{
Dissertation submitted \\ to the School of Medicine \\ at West Virginia University \\ in partial fulfillment of the requirements for the degree of \\ Doctor of Philosophy in \\ Immunology and Microbial Pathogenesis
}

\author{
Rosana Schafer, PhD, Committee Chairperson \\ Christopher F. Cuff, PhD \\ Jeffrey S. Fedan, PhD \\ James M. Sheil, PhD \\ Paul D. Siegel, PhD \\ Jenny R. Roberts, PhD, Mentor \\ Department of Microbiology, Immunology, and Cell Biology \\ Morgantown, West Virginia \\ 2017
}

Keywords: crystalline silica, diesel particulate matter, occupational exposure, pulmonary, toxicity, inflammation, immunological response, fibrosis

Copyright 2017 Breanne Yingling Farris 


\title{
Abstract \\ An in vivo evaluation of pulmonary immune response and toxicity following combined respiratory exposure to crystalline silica and diesel particulate matter
}

\author{
Breanne Yingling Farris
}

Crystalline silica (SIL) and diesel particulate matter (DPM) are each well-recognized for their potential to cause pulmonary toxicity following inhalation. However, these particulates have also been identified as coexisting in mixtures of respirable aerosols at a variety of industrial settings: mining, oil and gas extraction, construction, road construction, tunneling, excavation, foundry, and sandblasting. In order to characterize the potential for increased pulmonary health effects when these particulates are inhaled in a mixture, a battery of acute and subacute in vivo exposure studies were conducted. The current studies examined co-exposure to SIL and DPM at doses that were derived from field measures collected during industrial activities. The project had three major aims: (1a) Characterize the pulmonary response to an acute co-exposure to DPM and SIL at varying doses; (1b) characterize the pulmonary response to a repeated coexposure to DPM and SIL in order to determine if subacute exposure vs. acute exposure altered the effects observed for 1a; (2) establish if susceptibility to an acute respiratory infection was altered following a co-exposure of DPM and SIL; and (3) determine whether clearance of particulate in a co-exposure to DPM and SIL was altered compared with the clearance rate of either particle individually to assess the effect of particle load in toxicity. Co-exposure of rats to SIL and DPM had the capability to enhance indicators of inflammation, injury, oxidant production by phagocytes, onset of the initiation of fibrosis, and differentially alter immunological responses when administered in combination, compared to either particle individually. In acute infection models examining respiratory susceptibility following exposure to particles, the highest dose of DPM was shown to suppress bacterial clearance in the innate phase of the immune response as compared to SIL. It was determined that although some macrophage functions were reduced by the presence of DPM early in the time course (1 to 3 days following infection), all groups cleared the infection at a similar rate in the adaptive phase of the immune response. Further, it was determined that some effects in the co-exposure group were associated with the presence of SIL and the overall particle load over time, particularly tissue remodeling and the development of fibrosis; whereas other effects were due to specific characteristics of DPM including inflammation, injury, and oxidant production. Overall, the studies indicate that silicaexposed workers may be at an increased risk for adverse health effects when concomitantly exposed to diesel particulate, even doses that cause low toxicity in individual exposures. 


\section{Dedication}

This dissertation is dedicated to my steadfast and beloved husband, Joshua, without whose unfailing support and encouragement throughout my baccalaureate and doctoral education I surely would not have succeeded. I would also like to dedicate this work to my loving and inspiring parents, Richard and Susan Yingling, to my devoted sisters, Collette Sites and Jerica Yingling, and to my adoring nieces, Aida and Savannah Sites. I would like to thank my grandfather, Wayne Diederich for his continuing encouragement and confidence in the pursuit of my education. I would like also to thank my father and mother-in-law, Mickey and Gená Farris, as well as my brother and sister-in-law, Michael Farris and Sun-Hee Park for their constant care and advice. I owe my continuing gratitude to the dedication of all of my family and friends without whom I surely would have failed. They have not only been faithful to celebrate my accomplishments, but also to endure my frustrations through the inevitable failures that accompany the pursuit of the advancement of science. I would also like to dedicate this work to the loving memory of my grandmother, Colleen Diederich, who believed that all people, especially women should pursue their education to the fullest of their ability, and whose valiant battle with cancer inspired me to pursue my dream of biomedical research and the understanding of the human immune system. Finally, this work is dedicated to God, the divinely inspired author of my story and of all things, without whom there is no purpose, knowledge, or greater understanding. 


\section{Acknowledgements}

Jenny R. Roberts for her dedicated mentorship, inspiration, and innovation and for demonstrating time and again her passion for research. Dr. Roberts has continually encouraged me and driven me to excel in the pursuit of my career.

I would like to thank James Antonini, Mark Barger, Terence Meighan, the Histopathology Staff, and the Animal Care Staff at NIOSH, for their integral assistance and training in the completion of hours of tedious animal work.

Fred Minnear for his acceptance of me into the program and continued support during my graduate work.

My thesis committee including Rosana Schafer, Christopher Cuff, Jeffrey Fedan, James Sheil, and Paul Siegel who were consistent in their provision of insight and improvements to my study design throughout my time in graduate school.

Special thanks to the instructors who paved the road of scientific curiosity and were essential to my pursuit of a scientific career including but not limited to Terry Martin, Dale Burnside, Stephen Scott, Marsha Fanning, and Karen McDougal, who also provided phenomenal undergraduate mentoring.

I would also like to thank Catherine Schein and Thomas Wood for their passionate guidance in the conduct of my undergraduate research that provided pivotal and meaningful experience leading to the pursuit of my graduate education.

A special thanks to my friends and lab mates; Carrie Long, Katie Roach, Kristen Russ, Danielle Shepherd, The McLaughlins, and Cindy McMillen who provided not only advice and support but also assistance in the planning and completion of experiments.

Finally, I would like to thank the Graduate Program for Biomedical Research at West Virginia University School of Medicine and the National Institute for Occupational Health and Safety for both financial support to attain my degree as well as consistent mentorship from faculty, staff, and peers during my training. 
Table of Contents

Title: An in vivo evaluation of pulmonary immune response and toxicity following combined respiratory exposure to crystalline silica and diesel particulate matter

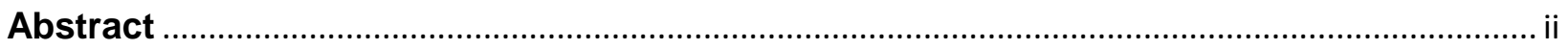

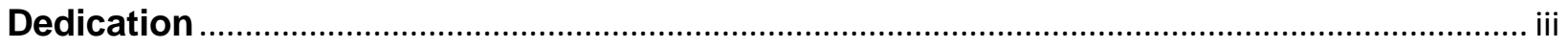

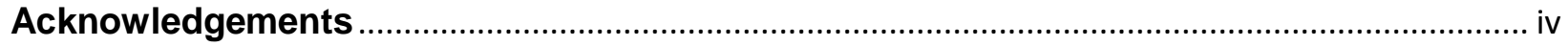

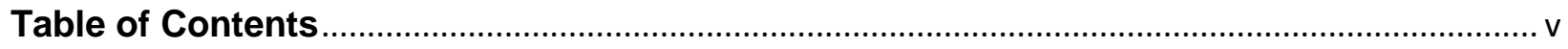

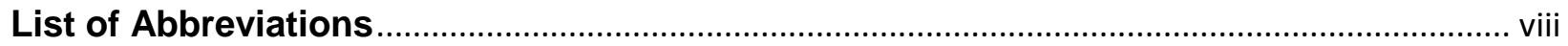

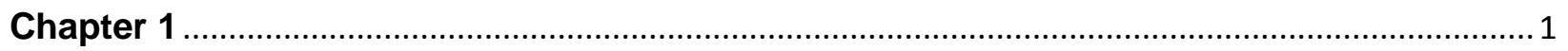

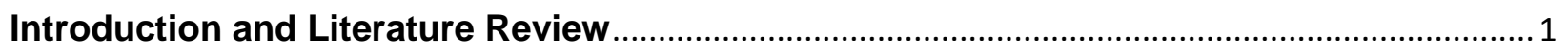

Human Health Effects of Respiratory Exposure to Crystalline Silica ................................. 3

Effects of Respiratory Exposure to Crystalline Silica in In Vivo Models .......................... 9

In Vitro Models of Silica-Induced Toxicity ......................................................................... 16

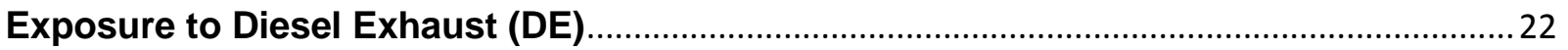

Respiratory Effects of Work-Place Exposure to DE …...................................................... 23

Respiratory Effects of Environmental Exposures to DE ...............................................26

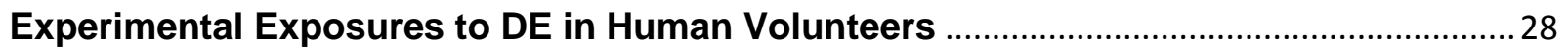

Effects of Respiratory Exposure to Diesel Exhaust in in vivo Animal Models.................32

In Vitro Models of Diesel Exhaust Toxicity ...................................................................... 44

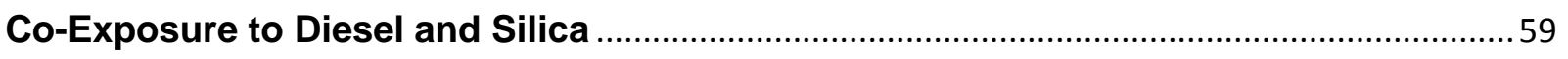

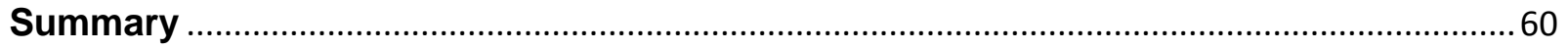

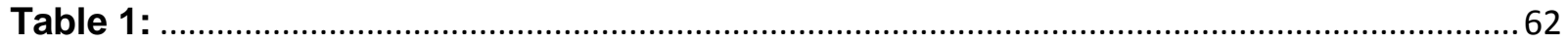

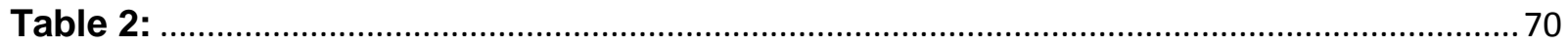

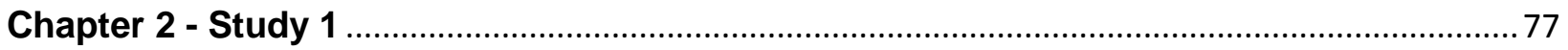

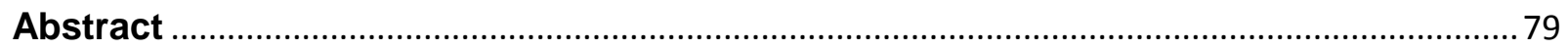

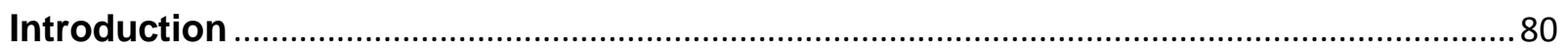

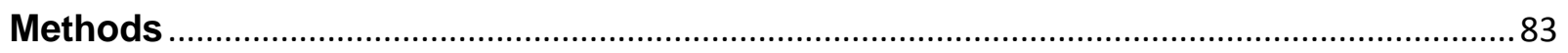

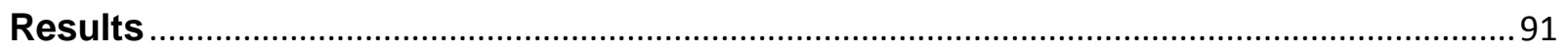

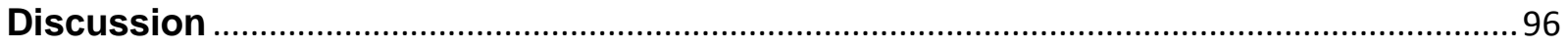

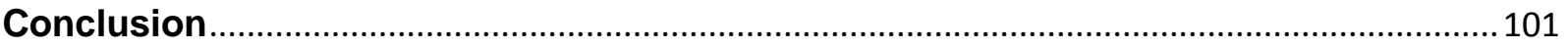

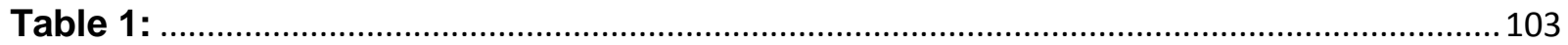




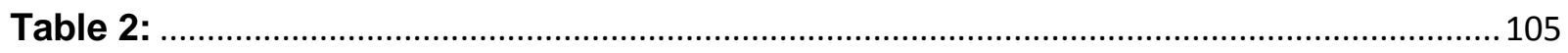

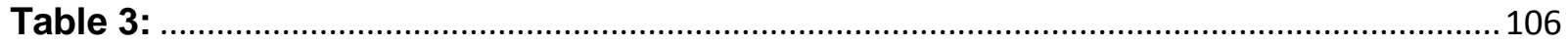

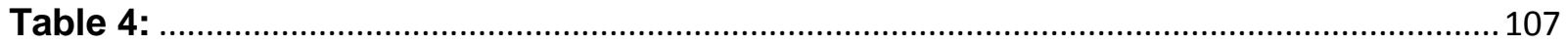

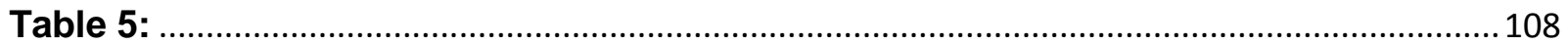

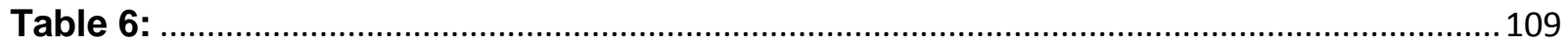

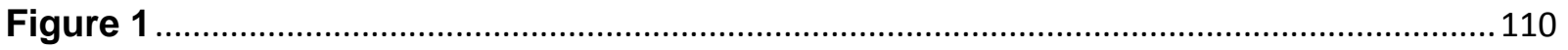

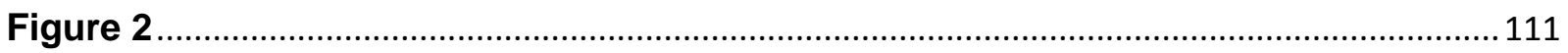

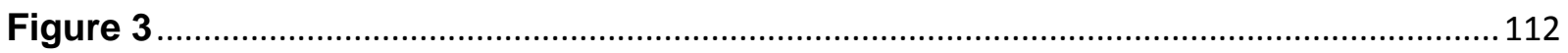

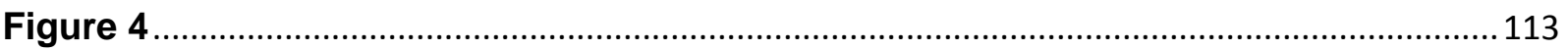

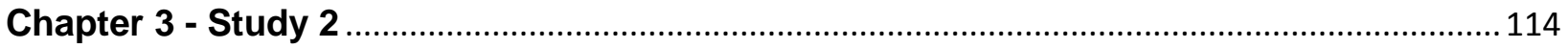

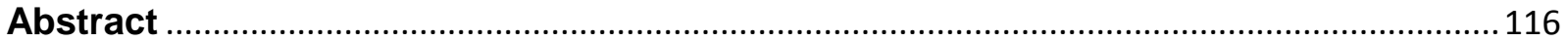

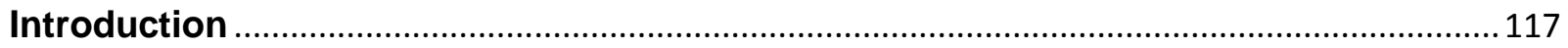

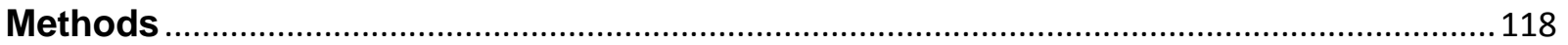

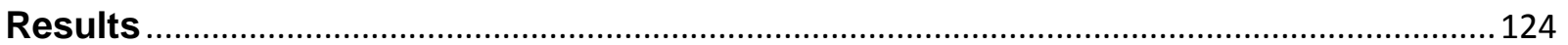

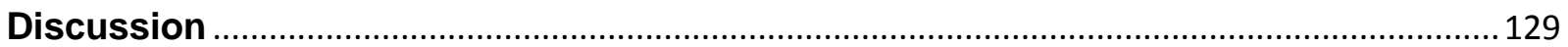

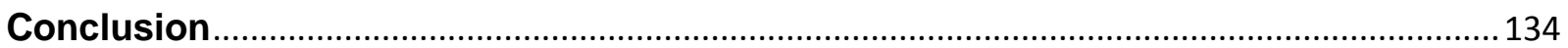

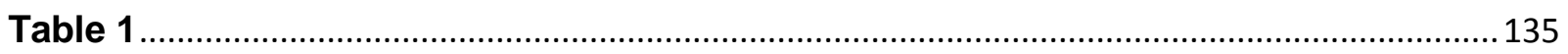

Table 2

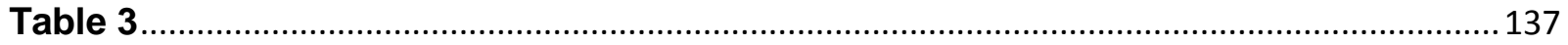

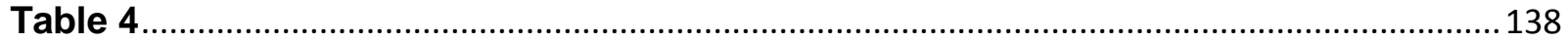

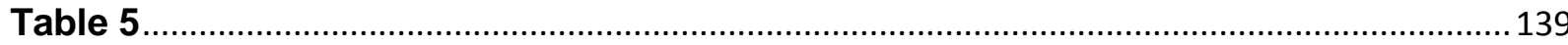

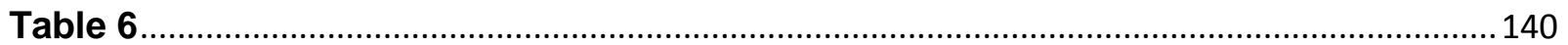

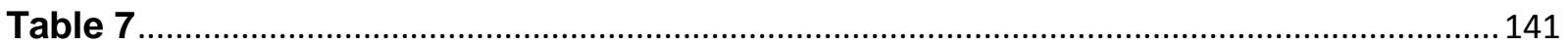

Table 8

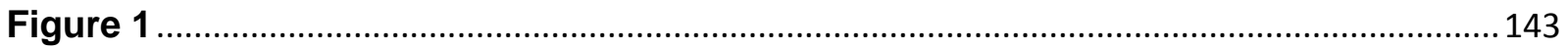

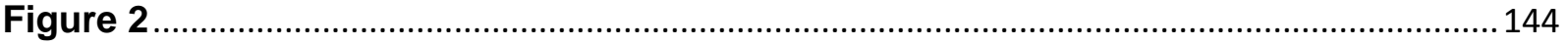

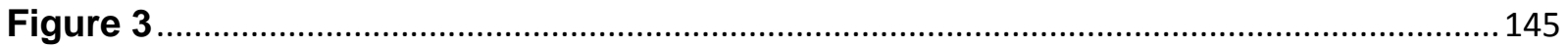

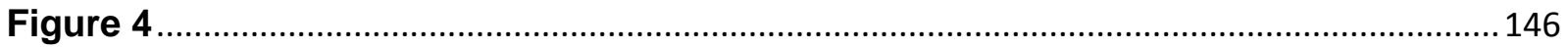

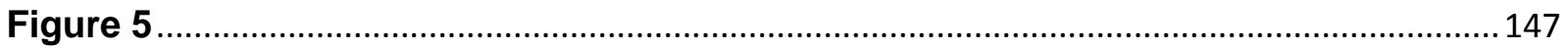

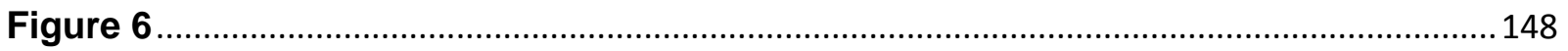

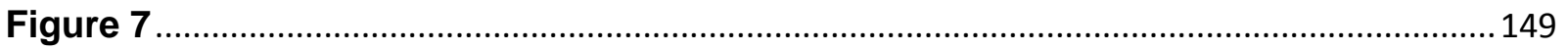

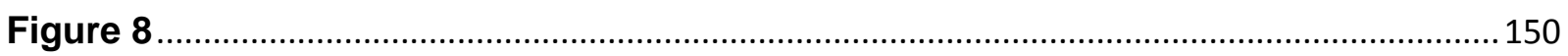

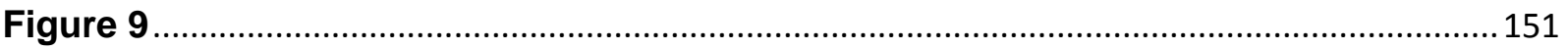




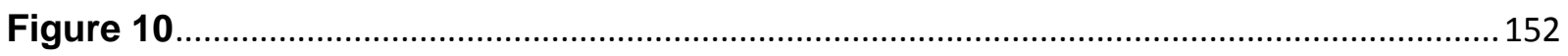

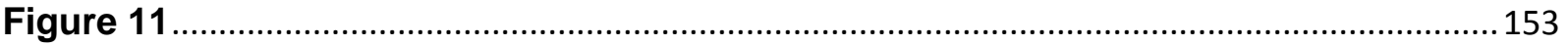

Figure 12

Figure 13

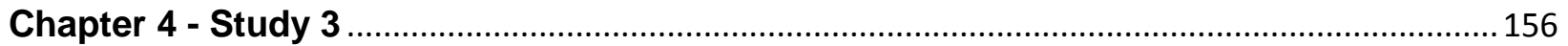

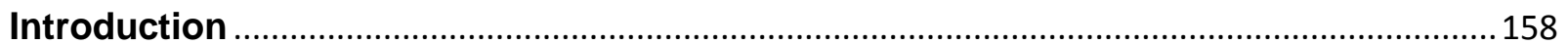

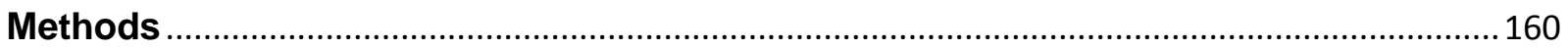

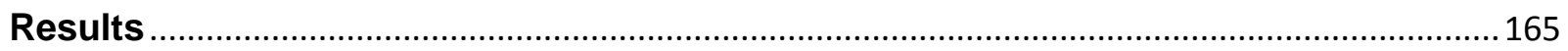

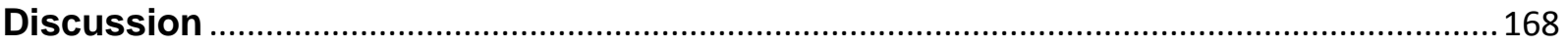

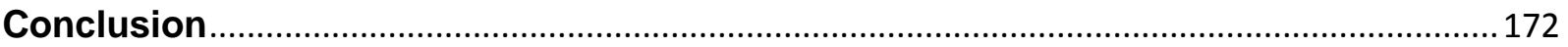

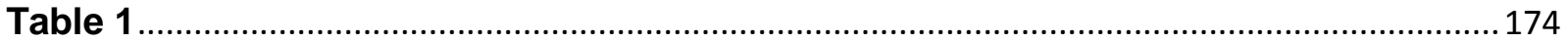

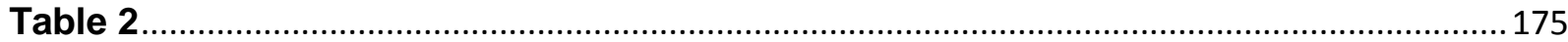

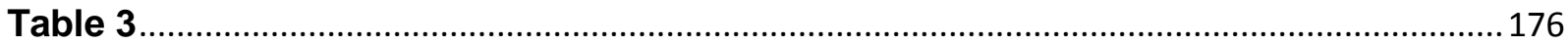

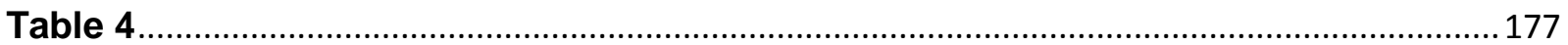

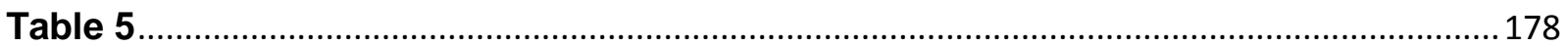

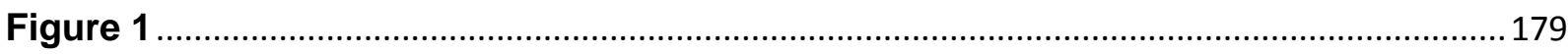

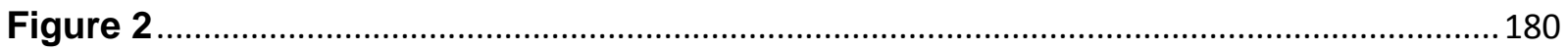

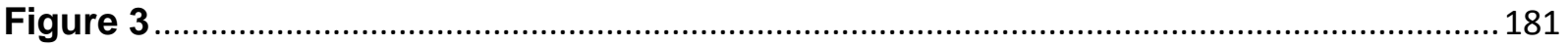

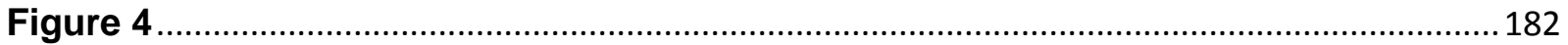

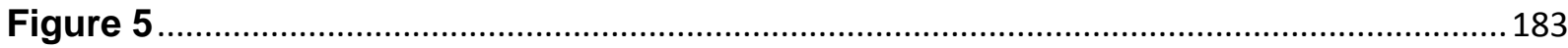

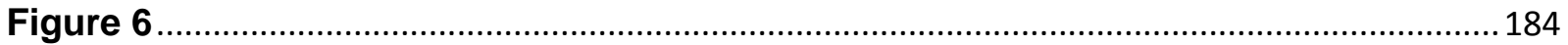

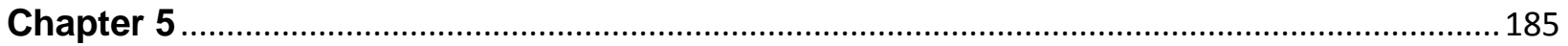

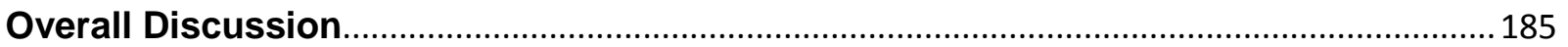

Model for Overall Progression of Lung Toxicity................................................................. 192

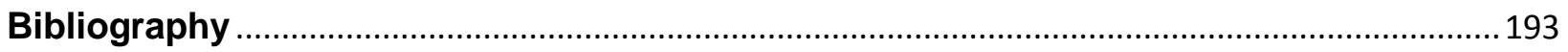

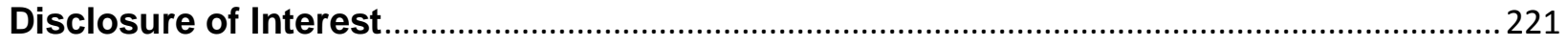

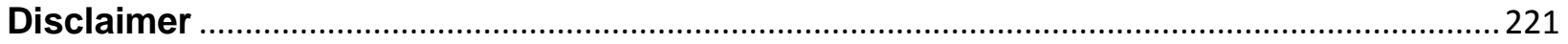

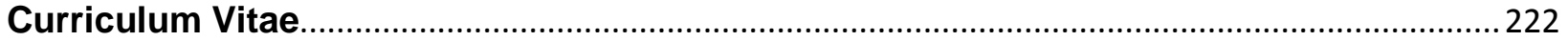




\section{List of Abbreviations}

8-OHdG 8-Hydroxy-2'-deoxyguanosine

9,10-PQ 9, 10-Phenanthrenequinone

ahR Aryl hydrocarbon Receptor

AHR Airway Hyperreactivity

AKR Aldo-keto Reductase

AM Alveolar Macrophages

ANCA Anti-neutrophil Cytoplasmic Autoantibody

AP-1 Activator Protien-1

APC Antigen Presenting Cell

CAD Coronary Artery Disease

CB Carbon Black

CCSP Clara Cell Secretory Protein

COPD Chronic Obstructive Pulmonary Disorder

cox Cyclooxygenase

CREB CAMP Response Element-Binding

CYP Cytochrome p450

DAMP Danger-Associated Molecular Pattern

DC Dendritic Cell

DE Diesel Exhaust

DEP Diesel Exhaust Particulate

DEPE Diesel Exhaust Particulate Extract

DHA Docosahexaenoic Acid

DMSO Dimethylsulfoxide

DPM Diesel Particulate Matter

EC Elemental Carbon

EMT Epithelial-Mesenchymal Transition

eNOS Epithelial Nitric Oxide Synthase

ERK Extracellular-Signal Related Kinase 


\begin{tabular}{ll} 
ESR & Electron Spin Resonance \\
FENO & Fraction of Exhaled Nitric Oxide \\
FEV & Forced Expiratory Volume \\
GM-CSF & Granulocyte Macrophage Colony Stimulating Factor \\
GSTM1 & Gluatathione S-transferase-Mu1 \\
H1R & Histamine H1 Receptor \\
HDL & High Density Lipoproteins \\
HDM & House Dust Mite \\
HEO & Heavy Equipment Operators \\
HH & Highway and Heavy (construction) \\
HO-1 & Heme Oxygenase-1 \\
HRV & Heart Rate Variability \\
IARC & International Agency for Research on Cancer \\
IFN & Interferon \\
Ig & Immunoglobulin \\
IHD & Ischemic Heart Disease \\
IL & Interleukin \\
ILC2 & Innate Lymphoid Type 2 Cell \\
iNOS & Inducible Nitric Oxide Synthase \\
IP & Interferon-y Induced Protein \\
JNK & C-Jun N-terminal Kinase \\
KO & Knock-out \\
LM & Listeria monocytogenes \\
LPS & Lipopolysaccharide \\
MAP & Mitogen Activated Protein \\
MCP-1 & Monocyte Chemotactic Protein -1 \\
MDC & Monocyte-derived Chemokine \\
MEK & MAP/ERK Kinase \\
MI & Myocardial Infarct \\
\hline HE & \\
HE &
\end{tabular}




\begin{tabular}{|c|c|}
\hline miRNA & Micro Ribonucleaic acid \\
\hline MMP & Matrix Metalloproteinase \\
\hline mtRos & Mitochondrial Reactive Oxygen Species \\
\hline MUC & Mucin \\
\hline MVE & Motor Vehicle Exhaust \\
\hline NBA & 3-Nitrobenzathrone \\
\hline $\mathrm{NCl}-\mathrm{H} 292$ & Nasal Epithelium and Human Lung Mucoepidermoid Cells \\
\hline NEC & Nasal Epithelial Cells \\
\hline NF-E2 & Nuclear Factor-erythroid 2 \\
\hline NF-k $\boldsymbol{\beta}$ & Nuclear Factor Kappa-Light-Chain-Enhancer of Activated B Cells \\
\hline NIOSH & National Institute for Occupational Safety and Health \\
\hline NK & Natural Killer Cell \\
\hline NLRP3 & Nod-like Receptor Pyrin domain-containing 3 \\
\hline NO & Nitric Oxide \\
\hline $\mathrm{NO}_{2}$ & Nitrogen Dioxide \\
\hline NOS & Nitric Oxide Sythase \\
\hline n-PAH & Nitrated Polyaromatic Hydrocarbons \\
\hline NTDE & New Technology Diesel Exhaust \\
\hline NTM & Non-tuberculous Mycobacterial \\
\hline $\mathrm{O}_{3}$ & Ozone \\
\hline OCM & Organic Carbon Matter \\
\hline ODN & Oligodeoxynucleotide \\
\hline $\mathrm{OH}$ & Hydroxyl \\
\hline OSHA & Occupational Safety and Health Administration \\
\hline OVA & Ovalbumin \\
\hline PA & Pseudomonas aeruginosa \\
\hline PAH & Polyaromatic Hydrocarbon \\
\hline PAMP & Pathogen-Associated Molecular Pattern \\
\hline PAR & Proteinase Activated Receptor \\
\hline
\end{tabular}




$\begin{array}{ll}\text { PDGF } & \text { Platelet-derived Growth Factor } \\ \text { PEG-SOD } & \text { Polyethyleneglycol-conjugated Superoxide Dismutase } \\ \text { PG } & \text { Prostaglandin } \\ \text { PM }_{2.5} & \text { Particulate Matter Diameter Less Than 2.5 Micrometers } \\ \text { PM }_{10} & \text { Particulate Matter Diameter Less Than 10 Micrometers } \\ \text { PMF } & \text { Progressive Massive Fibrosis } \\ \text { PMN } & \text { Polymorphonuclear Cells } \\ \text { PSS } & \text { Progressive Systemic Sclerosis } \\ \text { PTEN } & \text { Phosphatase and Tensin Homolog } \\ \text { PVNO } & \text { Polyvinylpyridine-N-oxide } \\ \text { RA } & \text { Rheumatoid Arthritis } \\ \text { REL } & \text { Recommended Exposure Limit } \\ \text { ROS } & \text { Reactive Oxygen Species } \\ \text { RSV } & \text { Respiratory Syncytial Virus } \\ \text { SC } & \text { Scleroderma } \\ \text { SCE } & \text { Sister Chromatid Exchanges } \\ \text { SLE } & \text { Systemic Lupus Erythematosus } \\ \text { SOD } & \text { Superoxide Dismutase } \\ \text { SP-A } & \text { Surfactant Protein A } \\ \text { SR } & \text { Scavenger Receptor } \\ \text { SSc } & \text { Systemic Sclerosis } \\ \text { TB } & \text { Tuberculosis } \\ \text { TC } & \text { Total Carbon } \\ \text { TF } & \text { Tissue Factor } \\ \text { TGF } & \text { Transforming Growth Factor } \\ \text { Th1 } & \text { Thelper cell Type 1 } \\ \text { Th2 } & \text { Thelper cell Type 2 } \\ \text { TLR } & \text { Titanium Dioxide } \\ \end{array}$




$\begin{array}{ll}\text { TLV } & \text { Threshold Limit Value } \\ \text { TNF } & \text { Tumor Necrosis Factor } \\ \text { Treg } & \text { T regulatory cell } \\ \text { TRPA-1 } & \text { Transient Receptor Potential Ankyrin 1 } \\ \text { TRPV-1 } & \text { Transient Receptor Potential Cation Channel } \\ & \text { Subfamily V Member 1 } \\ \text { TRPV-4 } & \text { Transient Receptor Potential Vanilloid 4 } \\ \text { TRX } & \text { Thioredoxin } \\ \text { TTF } & \text { Thyroid Transcription Factor } \\ \text { TW } & \text { Tunnel Workers } \\ \text { TWA } & \text { Time-Weighted Average } \\ \text { UBQLN } & \text { Ubiquilin } \\ \text { UPR } & \text { Unfolded Protein Response } \\ \text { VOC } & \text { Volatile Organic Compounds } \\ \text { wDEP } & \text { Washed Diesel Exhaust Particles } \\ \text { WT } & \text { Wild-type } \\ \text { ZO-1 } & \text { Zonular Occludin-1 }\end{array}$


Chapter 1

\section{Introduction and Literature Review}




\section{Literature Review}

\section{Introduction}

Exposure to airborne particulate occurs in a variety of occupations. Recorded observation of respiratory diseases resulting from such exposures date back to prehistoric eras, including mining with origins as far back as the Paleolithic and Neolithic eras [1]. It is speculated that silicosis, once referred to as "dust phthisis" and "knapper's rot," a pneumoconiosis caused by the inhalation of crystalline silica dust, may be the oldest known occupationally acquired disease, dating back to the flint mining and knapping origins of industry [2]. Silicosis is still prevalent to date, associated with a number of different occupations including mining, and data from the Coal Worker's Health Surveillance Program (CWHSP) suggests it is again on the rise in coal miners, after a period of decline in the 1980's [3]. Silica exposure has been shown to lead to several health complications, most notably progressive fibrosis and cancer [4]. In addition to silica, workers in the oil and gas industry are also at risk for exposure to diesel exhaust. The diesel engine, invented in the 1890s, was used mostly for industrial or publicsector transportation applications until its transformation for private use in the 1980s. Combustion exhaust was not recognized as a health hazard until the latter part of the $20^{\text {th }}$ century. Pulmonary exposure to diesel exhaust (DE) is now associated with several adverse health effects including but not limited to inflammation, decrements in lung function, chronic obstructive pulmonary disorder (COPD), bronchitis, asthma, cancer, respiratory infection, cardiovascular disease, and stroke [5, 6]. Components of diesel exhaust are complex and variable dependent upon many factors including the type of fuel combusted, the age and maintenance of the engine, and the temperature and pressure at which the engine is operated [5]. Due to the complex nature of diesel exhaust the approach to regulating occupational exposure has varied globally.

Co-exposure to respirable crystalline silica and diesel particulate matter (DPM) may occur in occupations in which diesel-powered equipment is primarily utilized for the purposes of disturbing the earth's crust, crushing, grinding, or polishing rock, as well as occupations in which the use of sand as a material is prevalent. These occupations include surface and underground mining, road paving and construction, tunneling, as well as masonry, ceramics, and sandblasting [7]. While the health hazards associated with each of these particles individually have been investigated, very few studies have systematically examined the possible risks posed by silica and DPM combined. Additionally, many of the in vivo studies examining these particles were conducted using doses that exceed real-time measures of particles in the personal 
breathing zones of workers collected during industrial activities. Because mixing particles may result in chemical interactions that alter their toxicological properties compared to the individual particles that constitute the mixture, and dose comparison to actual exposure measurements are of utmost importance in order to determine the combined risk of silica and DPM exposure in the workplace, it is necessary to investigate these two aspects for a better understanding of the possible adverse health outcomes that may result due to co-exposure to these materials in occupational settings.

\section{Human Health Effects of Respiratory Exposure to Crystalline Silica}

Currently, the Occupational Safety and Health Administration (OSHA) report that approximately 2 million workers in the United States are potentially exposed to crystalline silica and for many of those workers air levels may exceed recommended exposure limits (RELs) depending upon the industry [7,8]. Millions more are affected in middle-low income countries such as India, China, Brazil, and South Africa. In addition to silicosis, exposure to silica is related to other respiratory illnesses, which have been extensively reviewed, including COPD, emphysema, chronic bronchitis, development of respiratory infection, the overall declination of lung function and cancer [9-12].

Moreover, non-pulmonary effects of silica exposure have also been identified including the development of autoimmune diseases, malignancies outside of the respiratory tract [13-16], cardiovascular diseases [17, 18] such as cor pulmonale [19] and ischemia [20], and renal disease. For the purposes of the current studies, the review will focus on the development of respiratory conditions as they relate to silica exposure and particularly to prolonged inflammation and alterations in immune function.

\section{Pulmonary Disease and Lung Cancer}

Pulmonary effects following silica exposure in workers are well-established. Respiratory exposure to silica causes pneumoconiosis, more specifically, silicosis, which is a restrictive, fibrotic lung disease. Etiology depends upon dose/exposure and duration. The four types are: acute, accelerated, chronic, and complicated or conglomerate silicosis, where the latter may advance to progressive massive fibrosis (PMF) [4, 21, 22]. Silicotic fibrosis has been wellstudied in humans across several decades by medical survey, retrospective, and case study (Table 1), and though it has been recognized in the literature since it was described by Georgius 
Agricola in the mid-1500s as an occupationally relevant disease [23], it has only been clinically recognized in the past century.

Silicosis was a grossly neglected occupational outcome of dusty trades until what is considered one of the worst industrial disasters in the history of the United States, "The Hawk's Nest Incident." In an effort to redirect the New River for hydroelectric utilization, a 3-mile-long tunnel through a mountain face in West Virginia, and still one of the greatest engineering feats of modern civilization, was commissioned by the Union Carbide and Carbon Corporation in 1930. The rock face was comprised heavily of crystalline silica. Nearly 5,000 workers contributed to the project, 2500 of whom worked underground at some point during that year. Of the 2,500 men who worked underground, 1,500 would eventually die due to complications associated with silicosis. Within 5 years of the project completion, 764 men lost their lives to acute silicosis. Due to underreporting of this disease, it is suspected that these numbers are actually an underestimation of mortality. Prior to this tragedy, silicosis remained fairly undiagnosed as it was difficult to distinguish from other respiratory diseases with similar symptomology and presentation such as pneumonia and tuberculosis [24].

Following this and other occupational exposure incidents, efforts have been made to improve the detection and prevention of silicosis, particularly by the International Labour Organization that set the standard for Chest Radiograph determination of silicosis in 1930, which has undergone several revisions to its current form in 2011 [25]. Silicosis is characterized based on criteria including clinical presentation, exposure history, exclusion of other diagnoses, and finally of radiographic evidence of rounded opacities. Earlier and improved detection has also been enhanced by the use of Digital Thoracic Radiography and Thoracic Computed Tomography with High Resolution [26].

Pneumoconioses such as silicosis develop in the lung due to unconstrained inflammation that eventually results in the fibrosis. Specific mechanisms that relate to this process are still actively investigated; however, it is understood that phagocytosis of silica causes the production of reactive oxygen species (ROS). ROS initiates inflammatory processes, which lead to the death of silica-containing phagocytes resulting in reuptake of silica particles by new cells and the cycle is perpetuated [4, 21, 27, 28]. In an effort to control local inflammation, fibrotic mediators are secreted to sequester damage within the formation of a granuloma. These granulomas, also referred to as silicotic nodules, become areas that are no longer capable of air exchange and therefore cause restrictive fibrosis or areas where lung expansion by air is no 
longer possible. Furthermore, prolonged inflammation may also lead to the upregulation of pathways that result in carcinogenesis [21].

Silica is listed as a Group 1 carcinogen by the International Agency for Research on Cancer (IARC) [29]. In a retrospective study conducted by Dong et al. silica exposure in refractory workers was linked with a higher incidence in mortality from lung cancer [19]. These studies showed a correlation in the outcomes of silicosis and lung cancer that were dependent upon severity of silicosis and the latency since exposure. However, these findings were determined to be independent of smoking tenure. Attfield and Costello reported that increased incidence of lung cancer in granite workers was associated with duration and extent of silica exposure and suggested that the exposure regulations from OSHA of $1.0 \mathrm{mg} / \mathrm{m}^{3}$ over 8 hours as a time-weighted average (TWA) and the National Institute for Occupational Health (NIOSH), $0.05 \mathrm{mg} / \mathrm{m}^{3}$ as a TWA for respirable crystalline $\mathrm{SiO}_{2}[30,31]$ at that time, were not sufficient for protection of workers with a lifetime tenure for occupational exposure to silica [32]. Data published by Preller et al. indicated similarly that silica was a lung carcinogen and that confounding factors including smoking and exposure to asbestos, while associated with increased risk of cancer, did not singularly result in increased risks observed for silica-exposed workers, which were dependent on duration of exposure and overall cumulative exposure [33]. Liu et al. corroborated former findings in their extensive lung cancer risk assessment cohort examining over 34,000 workers in which they concluded that silica was a carcinogen, that exposure limits in many countries may be insufficient, and that smoking tenure increases risk for lung cancer when combined with silica exposure [34]. These findings were reaffirmed by Kachuri et al. in a population study of Canadian men where investigators found that exposure to silica regardless of silica source or smoking history was associated with an increase incidence of lung cancer. These findings were exposure duration dependent and additionally coincidence with smoking tenure increased risk beyond that of silica exposure alone [35]. While lung cancer dominates the carcinogenic findings associated with silica exposure, it does not stand alone as the only malignancy related effect.

Beyond the growing data implicating silica exposure in the incidence of lung cancer is the support for other malignancies such as upper airway neoplasms. In a case study conducted by Elci et al. data suggested that patients who had been exposed to silica dust had a higher incidence of laryngeal cancers specifically of the supraglottic region, and that this relationship was dose- dependent [36]. The same group reported consistently that silica exposure increased the incidence of laryngeal cancers in a group of non-smoking and non-drinking men, with the 
majority of cases occurring in the supraglottic region [37]. Laryngeal cancer was reported as occurring more frequently in those exposed to free respirable crystalline silica by Sartor et al. in a case study conducted in Brazil [38].

\section{Autoimmune Disease}

Silica exposure has been shown to have an increased association with disease resulting from autoimmune etiologies, as reviewed by Maeda et al. [39], although the American Thoracic Society recognizes that some of these associations may need further examination including: scleroderma (SC), systemic lupus erythematosus (SLE), and rheumatoid arthritis (RA) [40]. In 1995, during a prospective cross-sectional medical study of 764 patients admitted to a rheumatology clinic performed by Koeger et al., it was found that work-related exposure to silica coincided with autoimmune connective disorders in 24 patients who had been exposed to silica for at least three years including: progressive systemic sclerosis (PSS), RA, and SLE [41]. Similarly, Makol et al. reported that silicotic patients were at a significantly increased risk for connective tissue disorders such as SC and anti-neutrophil cytoplasmic autoantibody-related (ANCA) vasculitis but also an increased risk for RA and SLE in a retrospective medical survey covering patient data reported from 1985-2006 [42]. A retrospective study investigating data collected from past medical examinations in a large cohort conducted by Blanc et al. also showed increased risk for rheumatological diseases like RA, SLE, and SC in silica-exposed construction industry workers and that this risk was increased for ever-smokers. The authors used an exposure matrix to determine occupational exposure based on exposure estimations from the 1970s. The study included over 200,000 patients after exclusions were applied and over 35,000 non-exposed controls who performed office duties at similar job sites [43]. Beaudreuil et al. studied the hypothesis that silica exposure may result in increases in ANCA in the plasma resulting in diseases like vasculitis or nephritis. This group concluded that silica exposure was the only investigated hazard that resulted in a significant difference in the presence of ANCA compared to controls suggesting silica played a role in the development of ANCA-related autoimmunity [44]. Results from the studies of Shtraichman et al. suggest that those exposed to silica may even be at risk for mixed connective tissue disorder reporting that many patients have overlapping symptoms consistent with multiple autoimmunological diagnoses [45]. In a population-based case-control study, Parks et al. found an association between work-related silica exposure and incidence of SLE in a cohort of people from the Southeastern United States, $n=265$ SLE and $n=355$ control. Further, job histories were determined by blind assessments, and most dusty trades for this study pertained to agricultural 
work performed in sandy soil [46]. Bovenzi et al. conducted a case-referent population study of scleroderma patients from 1976-1991. The authors examined 21 patients with scleroderma compared to 42 matched controls, which suggested that among other occupational exposures, silica increased the risk for SC and systemic sclerosis (SSc). A minimum of six months of relevant exposure was required for inclusion [47].

Association of silica exposure with development of autoimmune disease has led investigators to examine how silica initiates this pathological process. One group has reported that soluble Fas, an alternatively spliced variant of the important autoimmune regulator Fas, is over-expressed in the serum of silicotics when compared with healthy donors. The increase in soluble Fas may lead to reduced apoptosis in aberrant immune cells resulting in a loss of selftolerance and production of autoantibodies [48, 49]. Furthermore, Wu et al. reported that T-cell activation occurring continuously by silica from consistent exposure, or buildup in the host, may cause activity of $T$ regulatory cells in peripheral blood to be reduced by replacement by activated T-cells that express CD4 and CD25. It was also noted that this data was collected prior to clinical autoimmune presentation suggesting that pools of regulatory $T$ cells may serve as a way to monitor autoimmune development and that silicosis may be a predetermining factor [50]. These findings led Otsuki et al. to hypothesize that there are two subpopulations of surviving silicosis lymphocytes: one, which is long surviving that recognizes self-antigen, and one, which undergoes apoptosis in response to silica and is sensitive to anti-fas autoantibody [51].

Studies conducted by Rocha-Parise et al. examined markers of immune activation in silica-exposed workers belonging to various industries: ceramics, mining, stonecutting, quarrying, and foundry among others. Peripheral blood was collected from 103 patients as well as 103 matched controls. Authors focused on the interleukin (IL)-2 axis where they report that patients exposed to silica had higher serum levels of IL-2 receptors and inflammatory cytokines such as IL-1 $\alpha$, IL-6, tumor necrosis factor (TNF)- $\alpha$, and interferon (IFN)- $\mathrm{y}$. The authors also examined immune cells and demonstrated that those from silica-exposed patients were much more proliferative than controls [52], suggesting hyperactivation of the immune system. In an earlier study Rocha et al. produced data that points to reduced self-tolerance due to polymorphisms that occur in the PD-1 and reduced expression of CTLA-4 involved in signaling that regulates apoptosis of autoreactive cells in silicosis patients [53]. Therefore, while the relationship of silica exposure and autoreactivity is well-characterized, the role of silica in altering immune response is not limited to autoimmune developments. 


\section{Infectious Disease}

The incidence of tuberculosis (TB) or infection with Mycobacterium tuberculosis is also reported to increase in individuals exposed to silica [54]. It has been recognized for centuries that exposure to dusty trade and phthisis or silicosis increases susceptibility to "consumption" or TB infection $[23,55,56]$. When both conditions occur simultaneously, it is known as silicotuberculosis, oftentimes presenting in non-classical ways, particularly fibroid without tendency toward cavitation, patients may present as sputum-negative despite radiological evidence of tuberculosis [57]. More recently, it was demonstrated that workers exposed to silica were more susceptible to TB infection even in the absence of silicosis, though susceptibility was increased with silicotic disease [58-62]. Though TB is the most common infection associated with silica exposure, there is evidence that chronic exposure to silica causes susceptibility to other infections as well.

Non-tuberculous mycobacterial (NTM) infections were reported in workers exposed to silica and most typically in those with pre-existing lung conditions such as pneumoconiosis, silicosis, or COPD. In a case study, Bailey et al. reported on various TB and NTM infections that occurred in silicotic sandblasters. These researchers concluded that these infections might be opportunistic due to reduced pulmonary defenses upon the development of occupational disease [55]. Schaefer et al. addressed NTM infections with an avian-Battey group that typically do not infect healthy adults. Their data suggested that damage to the lungs by industrial exposure was associated with opportunistic infection and typically poor prognosis [63]. In a review of NTM by Wolinsky, predisposing conditions most commonly associated with NTM infections include pneumoconiosis, previous TB infection, and COPD. Not surprisingly the investigator outlines silicosis as being related with NTM infection as documented in several studies [64]. Suzuki et al. more recently investigated atypical cases of $M$. scrofulaceum in adults, a pathogen more typically associated with childhood lymphadenitis. They concluded that chronic pulmonary disease was associated with this opportunistic infection and that literature covered most-noted history of dusty occupations and silica exposure [65]. Finally, it has been acknowledged that diagnosis with multiple and mixed pulmonary NTM is possible in patients with underlying pulmonary disease [66]. Furthermore, it should be noted that mycobacterial infections following silica exposure, while dominating the literature on silica-associated infections, are not exclusively reported.

Among other infections recorded for occupational exposure to silica include bacterial infections with nocardiosis [55] as well as infection with Acinetobacter. Cordes et al. examined 
three reported cases of non-nosocomial Acinetobacter calcoaceticus variety anitratus pneumonia that occurred among foundry workers, one of whom had a confirmed silicotic nodule upon post-mortem examination. It was stated that worker exposure to mixed dust, free silica, and metallic particles were in excessive levels. Specifically, free silica exceeded NIOSH REL. All three cases of pneumonia were of the same strain and were likely contracted by aerosol route [67]. Though bacterial infections have been more consistently associated with silica exposure, fungal infections are also recognized. Bailey et al. noted a few cases of sporotrichosis and crytococcosis known among sandblasters [55]. The importance of further investigations involving atypical tuberculosis was stressed by da Silva et al. in a report on a patient with concomitant silicosis and crytococcosis [68]. Though complete understanding of how silica affects the immune system remains elusive, and taking into consideration that these infections are known to cleared by cell-mediated mechanisms, it is likely that chronic exposure to silica impairs this response at least partially. Subra et al. showed that in individuals with pulmonary silicosis resulting from occupational exposure, circulating numbers of total lymphocytes were reduced when compared with controls [69]. Further, Schuyler et al. reported that lymphocytes recovered from silicotics were less responsive to low concentrations of concanavalin A [70]. Further studies are needed in order to determine the full effects of silica exposure with regard to cell-mediated immunity.

\section{Effects of Respiratory Exposure to Crystalline Silica in In Vivo Models}

\section{Pulmonary Effects}

Animal studies of silica exposure have been conducted over the past few decades. The study of silica exposure in vivo has not only led to support for epidemiological/human studies but has primarily elucidated possible mechanisms for disease progression. These studies include, but are not limited to, mechanism related to pulmonary clearance or lack there-of, oxidant-induced lung injury and inflammation, and the role of immune regulation in the development of fibrosis.

It is widely accepted that the initiation of toxicity in the host is directly related to uptake of silica particles by alveolar macrophages (AMs) and cytotoxicity on the path to progression towards fibrosis. Schapira et al. demonstrated that instillation of silica into rat lungs caused increased acute inflammation driven by the production of hydroxyl radicals produced in excess when compared to instillation of a less toxic material, titanium dioxide $\left(\mathrm{TiO}_{2}\right)$ [71]. In a later study, Shapira et al. showed that phagocytic cells ex vivo produced increased levels of nitric 
oxide (NO) following exposure to silica by increased uptake and metabolism of L-arginine, unveiling another potential mechanism for silica-induced ROS production in vivo [72]. In a series of studies examining silica inhalation exposure in rats, Porter et al. demonstrated that NO and ROS were increased in the lung following exposure to silica and that the production of these molecules was associated with pathological lesions in the lung caused by silica particle exposure [73]. Additionally, oxidative effects of silica continued after silica exposure ceased. Their results indicated that even after exposure ends and a period of recovery occurs, and though silica may be cleared from the lung below a specific threshold of exposure, although slowly, damage induced by silica particles continues to increase. [74]. It was also confirmed that these results were obtained for a level of exposure below the threshold for what would be considered to be "overload" [75]. Moreover, it was shown that this damage was mediated by oxidative stress resulting from increased presence of $\mathrm{NO}$ and ROS in the lung [76]. Sato et al. showed that progressive inflammation caused by ROS production upon exposure led to infiltration of cells into the lung and production of signaling molecules, but that these effects could be reduced by treating with oligodeoxynucleotides (ODN). The authors hypothesize that ODN suppress mitochondrial instability that contributes to the production of ROS as their data supported that ODN helped to maintain mitochondrial stability [77].

ROS is known to upregulate the activation of transcription factor, nuclear factor-kappa $B$ (NF-KB) [78, 79]. Consequently, Porter et al. [80] and Castranova et al. [81] investigated the effects of silica exposure on the activation of NF-KB. Data reported in this study concluded that the relationship between silica exposure in vivo and the degree of NF-kB activation was directly related to time beginning at 5 days of exposure and increasing steadily throughout the timecourse. Since fibrosis was not found to occur until a later phase of inflammation, it is reasonable to surmise that NF-KB may be involved in the development of fibrosis in the lung following silica exposure. Studies conducted by Zhu et al. showed imbalances of redox due to increased production of ROS and the down regulation of the thioredoxin (TRX) system resulted in oxidative stress, which may eventually lead to fibrosis [82]. Further, more recent investigations have delineated mechanisms that demonstrate that TRX may have a role in inflammasome regulation.

Peeters et al. investigated the role of TRX and inflammasome upregulation downstream of ROS production. They concluded that rats treated with crystalline silica particles had increased inflammasome activation indicated by increased activation of caspase- 1 and IL-1 $\beta$ [83]. Studies conducted by Ghio et al. focused on silica particle chemistry and the ability of silica 
to sequester iron leading to collagen deposition [84]. Supporting this mechanism, Shimizu et al. discovered that silica particles in mouse lungs were correlated with increased iron complexes. In addition to increased silica-iron complexes, accumulation of p62 was demonstrated in histiocytes, primarily shown to be macrophages, of granulomas. Increased levels of p62 are indicative of downregulation of autophagy. Decreased autophagy may allow for cell survival, and prolonged secretion of inflammatory and fibrogenic factors by these macrophages. Oxidative stress is initiated by iron-silica complexes, which may ultimately result in decreased cell death by autophagic mechanisms and hence silicotic progression. It should be noted, however, that a direct connection between increased iron-silica complexes and increased p62 have yet to be established [85]. Moreover, Jessop et al. elucidated that autophagy in vivo following silica exposure was at least in part responsible for the regulation of the inflammasome, and alterations in autophagy led to enhanced inflammation and cytotoxicity. Authors exposed autophagy deficient mice to silica, which led to increased production of inflammasome products including IL-18 in lavage fluid. These results highlight the importance of autophagy during homeostasis of cells following particle exposure/uptake [86].

Similar to autophagy, apoptosis has been implicated in the development of silicosis and lung cancer following exposure to silica. Upstream of the inflammasome in the proposed pathway, Moncao-Ribeiro et al. examined the role of $\mathrm{P} 2 \mathrm{X} 7$, a purinergic receptor that responds to extracellular ATP, in the development of silicosis. Their data supports the conclusion that P2X7 regulates silica-induced changes in the lung by responding to extracellular ATP produced in response to damage created by silica, and mediating the production of ROS, which in turn result in mitochondrial damage and eventually apoptosis and progressive inflammation [87]. Wang et al. investigated the role of the transcription factor p53 in apoptosis following silica exposure in animals and found that when p53 was knocked out, silica-induced apoptosis was diminished [88]. Adding to the evidence that increased apoptosis may play a role in the development of silicosis is a study performed by Borges et al. These investigators showed the importance of Fas ligand in the development of silicosis and the pro-inflammatory role of apoptosis following exposure to silica. The authors demonstrated that in mice lacking Fas ligand, macrophages failed to undergo apoptosis in response to silica exposure and neutrophil infiltration was reduced blunting the progression to prolonged inflammation and advancement to silicosis. These results were further supported by both the adoptive transfer of wild-type (WT) macrophages restoring these responses in Fas ligand knock-out (KO) mice and the blockade of silicosis induction using a Fas ligand antibody in WT mice [89]. 
Inflammation is an important initiating event modulating disease following silica exposure; therefore, research has emphasized the role of innate mechanisms following silica particle phagocytosis in disease. However, in light of the fact that silica has such a pronounced effect on cell-mediated immunity and disruption of proper antigen recognition, investigators have also focused on pathways that may be involved in toxicity including, at least in part, the development of fibrosis. Suzuki et al. report that, although T lymphocytes regulate a portion of fibrogenesis, athymic mice still developed fibrosis in response to silica administration, demonstrating that $T$ cell regulation is only partly involved [90]. Langley et al. report that there is a "biphasic" response to silica and while hyperresponsiveness of T cells occurs during the first of two phases, this is not required for the development of granulomas and progression to fibrosis in the second phase as adaptive immune responses were found to have returned to normal [91]. Migliaccio et al. determined the importance of antigen presenting cell (APC) phenotype shift and lymphocyte activation that may initiate prolonged inflammation. Treatment of mice with silica intranasally induced increased APC activities in AMs as wells as the expansion of a subset of interstitial macrophages with APC markers. It remains to be determined whether these cells are recruited to the lung or if activation of existent cells occurs due to exposures. However, it was hypothesized that these cells and the shift of their phenotype to increase APC activity were responsible for increased activation of T-cells and therefore initiation of inflammatory and pro-fibrotic mechanisms [92].

Due to previous findings that demonstrated the importance of T-helper Cell Type 1 (Th1) and Type 2 (Th2) cytokines in the development of silicosis and the known role for T regulatory lymphocytes (Tregs) for maintaining immune homeostasis, Liu et al. suggest that depleting Tregs might help to balance the Th1 and Th2 axis and shift response back toward Th1 from Th2 to evade fibrogenesis. They showed that Treg cells modulate inflammation in early stages by expression of CTLA-4, and IL-10 and transforming growth factor (TGF)- $\beta$ expression in later stages promote fibrosis [93]. Guo et al. examined the balance of the Th1/Th2 axis as well. Their data suggests that suppression of IL-1 $\beta$, a cytokine that is expressed following exposure to silica, and upregulation of the Nod-like Receptor, pyrin domain-containing 3 (NLRP3) inflammasome, may inhibit other fibrogenic mediators downstream of IL-1 $1 \beta$, such as collagen I and fibronectin, and offset prolonged inflammation leading to fibrosis. The authors blocked IL-1 $\beta$ using an antibody in silica-exposed mice and demonstrated that inhibition of this cytokine resulted in reductions in TNF- $\alpha$ and macrophage chemoattractants. Gene expression of important fibrogenic factors was also reduced by the blockade of IL-1 $\beta$ [94]. Chen et al. reported that neutralization of a different inflammatory cytokine, IL-17, known to be involved in the 
progression of autoimmune responses, resulted in reduced initial phase inflammation in response to silica. They found that blockade of IL-17A resulted in decreased IL- 6 and IL-1 $\beta$ following silica exposure in mice and, therefore, a reduction in acute phase inflammation. The authors hypothesize that IL-17A plays a role in the silica-initiated disruption of Th1/Th2 homeostasis leading to fibrosis [95]. Another group led by Li explored the possibility that 4-1BB, a co-stimulatory molecule in T cell activation might be involved in the development of silicosis. Li et al. reported that blockade of 4-1BB reduced fibrosis by controlling the initial Th1 driven inflammatory response resulting in a decreased Th2 response following initial inflammation [96]. Altogether these data suggest dual phase response of the immune system following silica exposure, which results first in hyperactivity of Th lymphocytes that may be induced by increased and highly active macrophages and, secondly, an uncontrolled progression of inflammation and fibrogenesis that is likely in part modulated by the inflammasome with a role for Treg cells.

Although most in vivo studies have emphasized the development of restrictive lung disease, fibrosis, studies also have aimed to elucidate the mechanisms that result in obstructive lung disease following exposure to crystalline silica. Wright et al. examined quartz exposure in rats and determined that in an animal model, quartz was capable of not only inducing silicosis but also obstructive lung disease characteristics including morphologic alterations to small airway tissue and functional changes consistent with emphysema [97]. Results from studies by Li et al. demonstrated that changes observed in the former study by Wright et al. may be due to destruction of elastic fibers that may be due to neutrophilic-derived enzymes. It was concluded that loss of this form of connective tissue played a role in the development of silica-induced emphysema [98, 99].

Perturbations of connective tissue by silica may also be implicated in the development of cardiopulmonary disease. Herget et al. showed that quartz caused pulmonary hypertension and increased heart ventricle weights [100]. Similarly, Zelko et al. showed that vascular remodeling, endothelial dysfunction, and inflammation were responsible for pulmonary hypertension in mice following intratracheal instillation (IT) exposure to silica [101]. Elevations in mRNA of inflammatory mediators and matrix proteins were observed in the heart and kidney of rats exposed to silica by Guo et al. Additionally, it was shown that neutralization of IL-1 $1 \beta$ decreased these fibrotic markers similar to their earlier studies conducted on the pulmonary tract [102]. 


\section{Systemic Immune Effects}

There have also been extensive studies in animals investigating correlations of silica exposure with systemic disease and autoimmune diseases, as in the case of ANCA. Autoimmune diseases, such as SLE and SSc, have been shown to occur in populations susceptible to the production of autoantibodies when exposed to silica. Brown et al. examined silica exposure in New Zealand mixed mice, a strain prone to lupus, to determine if a predisposed population exposed to silica might develop autoimmune disease. This group showed that silica significantly exacerbated the course of SLE development in these mice, indicated by increased mortality, proteinuria, circulating immune complexes and autoantibodies compared with saline and $\mathrm{TiO}_{2}$ control treated animals [103]. Additionally, mice had increased inflammation collagen deposition in the lung consistent with pulmonary fibrosis. The same investigators showed in a later study that alterations in populations of lymphocytes, primarily increased $\mathrm{CD} 4^{+} \mathrm{T}$ cells, resulted in reduced overall numbers of Tregs, which may allow for autoreactivity [104]. In experiments conducted by Pfau et al. it was determined that silicainduced apoptosis led to autoimmune response, by demonstrating that when apoptosis was blocked, increased binding of autoantibodies in mice treated with silica was inhibited [105]. Brown et al. went on to investigate the hypothesis that silica-induced apoptosis in AMs contributed to the generation of autoantibodies and showed that reduction of apoptosis by treatment with rottlerin decreased autoimmunity related to silica-exposure [106]. Further, Strickland et al. examined the effect of oxidants on $\mathrm{CD} 4^{+} \mathrm{T}$ cells to determine if this may be a cause for lupus-like autoimmune developments in animals exposed to agents that cause oxidative damage. Adoptive transfer of these oxidant-exposed T cells, which were shown to overexpress CD40L cause anti-DNA antibodies and result in lupus flares [107]. These findings demonstrate that silica exposure may lead to altered DNA methylation by production of oxidants resulting in autoimmune developments in susceptible populations.

Bates et al. examined repeated, short-term silica exposure on SLE-susceptible mice and found that silica induced pulmonary inflammation and elevated cellular infiltrates to the lung suggestive of ectopic lymphoid tissue development. Additionally, this group showed that silica exposure led to systemic inflammation and initiation of pathogenic mechanism in the kidney consistent with glomerulonephritis [108]. The same investigators also showed in a later study that the influence of diet, which alters the lipidome, may play a role in off-setting mechanisms that promote silica-induced autoimmune development indicating that certain populations may be at a greater risk for the initiation of autoimmune disease and recurring flares following silica 
exposure dependent upon balance of the lipidome. They found that consumption of a polyunsaturated fatty acid, docosahexaenoic acid (DHA) may prevent onset of autoimmunity. Treatment with DHA or consumption of DHA in susceptible populations may help to reduce or delay the onset of autoimmune disease [109]. Ultimately, it has been demonstrated that silica results in autoimmunity through apoptotic mechanisms, which increase production of autoreactive antibodies, and also shifts in normal circulating $T$ cell populations.

\section{Susceptibility to Infectious Disease}

Mechanisms elucidating susceptibility to mycobacterial infection following silica exposure are not well investigated due to the lack of adequate animal model for investigation until recently, because an increase in susceptibility to mycobacterial infection due to silica exposure could not be demonstrated in vivo [110]. Interestingly, past correlations have been drawn between human susceptibility to tuberculosis infection following exposure to silica and animal susceptibility to infection. Badgers have always been considered to be inherently susceptible to mycobacterial infection. Higgins et al. elucidated a possible connection with environmental exposures to silica and propensity for disease through the discovery of silicotic fibrosis developments in the lungs of wild badgers upon post-mortem examination [111]. While it has been recognized for several centuries that those employed in dusty trades had a higher incidence of infectious diseases like tuberculosis, models examining infectious disease as it relates to occupational silica exposure have only been developed in vivo within the past few decades. Hatch et al. investigated variable occupational exposures in mice, and their susceptibility to infection following exposure. The authors reported that for silica, there was only a slight increase in infectivity, though this study was conducted following acute exposure to a low dose of silica $(100 \mu \mathrm{g})$ and only mortality was used as an end-point for infectivity [112]. Pasula et al. describe one of the first studies conducted in mice in which they uncovered a mechanism for silica to alter macrophage populations in a way that favors mycobacterial persistence in the host [110] to model silicotuberculosis. Conversely, Antonini et al. showed that short-term silica exposure resulted in enhanced microbial clearance owed to the increase in cellular recruitment to the lung and production of oxidants likely aiding in bacterial clearance [113]. Antonini et al. state that in a chronic study of silica exposure host defenses were impaired requiring further examination of silica-exposure and the importance of time in relationship to exposure and infection.

In vivo work has been critical in helping to elucidate mechanisms of disease associated with silica exposure, but also of value are the vast number of studies that have been performed 
in vitro. Many of the limitations involving in vivo work relate to inadequate modeling of physiological exposure of animals to silica, time-constraints, dose constraints for modeling exposure, or insufficient techniques for examining intricate mechanisms within a host; therefore, cellular studies have also been useful and have provided insights into the mechanisms of disease.

\section{In Vitro Models of Silica-Induced Toxicity}

It has been shown that depending upon model of animal used, responses to silica varied significantly. Huaux and Barbarin et al. investigated the release of cytokines by AMs following exposure to silica and reported species-dependent expression of TNF- $\alpha[114,115]$. Dorger et al. examined the release of $\mathrm{NO}$ from macrophages in response to stimulation/activation by pulmonary irritants and found species dependent results [116]. Silica elicited fibrotic responses varied between models also, with rats being the most sensitive to pro-inflammatory changes induced by silica [117, 118]. These studies suggest that responses in biological systems may vary dependent upon species. Additional studies in cellular systems including human cell lines have been beneficial in advancing the field, particularly with regard to mechanisms of direct particle-cell interaction.

\section{Pulmonary Toxicology}

Although most work contributing to the expansive body of silica-exposure related studies has focused on addressing silicotic developments in the pulmonary tract, there are studies examining other detrimental effects of silica previously mentioned including cardiovascular disease [119]. Although, for the purposes of this review, emphasis in the in vitro section will be focused on pulmonary related effects and mechanisms involved in immunity to respiratory infection.

The mechanisms of action in silica toxicology and pathology have yet to be fully elucidated, although investigators have outlined several critical events in silica toxicity, which are widely accepted including interaction with resident alveolar macrophages [120,121] followed by sustained inflammatory cascades resulting from macrophage mediation [122, 123], recruitment of additional cells [124-126], further activation of the immune system [127-129], and cell death $[130,131]$. Research on particle-cell interactions has led to 4 basic overarching mechanisms for toxicity [27]. While an overwhelming body of literature exists addressing 
specific aspects of crystalline silica toxicity, most of this work is aimed at examining one of these broad categories.

1) Cytotoxicity of Lung Cells Caused by Silica Particles Directly

The principle that crystalline silica causes direct cellular toxicity resulting in the creation of cytotoxic mediators [4] is well-established. There are possible mechanisms that address the toxicity of silica particles relating to the surface chemistry and the reactivity of $\mathrm{SiO}_{2}$ when in direct contact with cells. 1) Free radical groups are formed when the surface of silica contacts aqueous solutions causing direct damage to biological membranes by lipid peroxidation. Nash et al. concluded that the toxic effects of silica are related the ability of silica to form complexes with structures such as phospholipids within membranes and act as a hydrogen donor [132]. Shi et al. showed that these effects were more significant in freshly fractured silica particles compared to those aged in aqueous solution [133]. Dalal et al. further examined this reaction and showed that radicals were likely the initiating step in the peroxidation of lipids caused by silica particles [134]. 2) Negative charges on the surface of silica interact with scavenger receptors on macrophages resulting in the production of ROS. Kobzik et al. found that anionic particles like silica were taken up by scavenger receptors (SR) on AMs. When SR were inhibited a reduced binding occurred [120]. Research by lyer et al. examined the potential of silica particles to initiate apoptosis in AMs leading to the release of fibrogenic factors. Blockade of either SR or downstream mediators of apoptosis resulted in reduced apoptosis and release of IL-1 $\beta$ from silica treated AMs [131]. 3) Fracturing silica creates Si radicals on the particle surface resulting in oxidant damage. Vallyathan et al. compared free-radical concentrations in freshly ground silica with aged silica by using electron spin resonance (ESR). Freshly ground silica produced silicon radicals that decreased over time in air and more rapidly in aqueous solution. Introduction of silica particles to aqueous solution increases the biological reactivity by methods described above. Further, freshly crushed silica induced greater lipid peroxidation as demonstrated by Castranova [135]. Experiments that suggested surface chemistry of silica as the initiating step in biological toxicity were further confirmed by research that showed that coating silica particles with substances such as organosilane [136], aluminum [137], polyvinylpyridine-N-oxide (PVNO) [132], or surfactant phospholipid [138, 139] resulted in reduced toxicity. Though direct cytotoxicity to AMs likely initiates mechanisms of chronic inflammation and fibrogenesis, there is a body of work that described additional mechanisms that are crucial for the development of silicosis and silica-related pathogenesis. The reaction of AMs and other phagocytes is also critical for the development of chronic inflammation. 


\section{2) Activation of Phagocytes and their Release of Oxidants}

Upon activation, AMs and polymorphonuclear cells (PMNs) or neutrophils release oxidants in a 'respiratory burst' prior to phagocytosing particles, as reviewed by Badwey and Karnovsky, Babior, and Fubini and Hubbard [130, 140, 141] and as demonstrated by Zhang et al. [142]. Vallyathan et al. showed that occupational particles including silica, particularly freshly fractured crystalline silica, enhanced the respiratory burst of phagocytes [28]. Also, upon phagocytosing silica materials, production of oxidants continued through effects on mitochondrial function resulting in generation of mitrochondrial ROS (mtROS). Mintz determined that phagocytosis of silica increased the mitochondrial redox state compared with the uptake of inert beads [143]. Fazzi et al. also have shown that reduction of mtROS may lead to reduced apoptosis of macrophages due to silica exposure and overall reduction in pathogenesis [144]. Additionally, it was suggested by Driscoll et al. that although cells have mechanisms that typically balance these redox reactions, particles that enhance production of ROS disturb this balance and may lead to increased cellular toxicity and mutagenesis of surrounding epithelial tissue, thereby recruiting additional phagocytes, which further perpetuates this cycle [145]. This may in part be due to a mechanism for chronic inflammation at the site of initial injury termed alveolitis. Lugano et al. showed in vitro that macrophages exposed to silica released more chemotactic factors for neutrophils and additional macrophages compared to control $[126,146]$. Furthermore, it is proposed that the release of reactive radicals by particles and ROS production by phagocytes may interact to form additional toxic oxidant compounds contributing to cell cytotoxicity [147]. Pricop et al. have demonstrated that RO intermediates caused increased phagocytosis acting as a mechanism for further inflammation and enhanced phagocyte effector functions [148]. Further studies have indicated a role for additional ions such as iron and calcium in the modulation of oxidant production by phagocytes. Ghio et al. revealed that iron complexes on the surface of silica particles may influence their potential for stimulating respiratory burst and subsequent recruitment of cells by exposed macrophages [149]. Kim et al. investigated the suppression of the respiratory burst of phagocytes and found that protein kinases and intracellular calcium transport were both intimately involved in the activation and subsequent response of AMs to silica particles [150]. 
Additionally, ROS have been implicated in the initiation of several carcinogenic pathways as well. Vallyathan et al. highlighted the importance of the increased activation of a transcription factor for inflammatory pathways and cell adhesion, activator protein (AP)-1. AP-1 was significantly increased in cells exposed to freshly fractured silica. This effect was attenuated by treatment with an ROS scavenger, leading to the conclusion that ROS may be involved in the activation of AP-1 [151]. Gwinn et al. examined the role of a transcription factor, p53, in the regulation of gene expression as it relates to DNA repair in the context of oxidant related damage caused by silica, both fresh and aged. Following this study, it was concluded that silica, particularly freshly fractured and highly reactive, caused cellular damage, which if persistent may lead to the potential to elicit carcinogenesis [152]. Based on the evidence described above, oxidative stress response by phagocytes is a key step in the initiation of carcinogenesis and fibrosis; however, the cascade of subsequent cell signaling events has been shown to be equally pivotal.

3) Inflammation Caused by Immune Cell Signaling

Recent studies have shown that the respiratory burst triggered by silica particles results in an intricate, yet not fully elucidated, series of signaling events [153,154]. These signaling events are part of a normal innate immune response to pathogen-associated molecular patterns (PAMPs) and danger-associated molecular patterns (DAMPs) that trigger upregulations of inflammation mediated by a large multi-protein complex sensor, the inflammasome $[155,156]$. The studies described below focused on investigations aimed at delineating the role of inflammasome formation as a critical part of the signaling cascade that may lead to more chronic lung injury.

Alveolitis or chronic inflammation caused by the influx of immune cells into the lung following exposure to particles is initiated by resident macrophages in an attempt to clear directly cytotoxic particles from the lung [157]; nevertheless, other immune and pulmonary cells also contribute to progression toward chronic inflammation. Beamer and Holian reported that dendritic cells (DCs) may be important for the production of some inflammatory cytokines in this progression [158]. Lo Re et al. have shown that $T$ lymphocytes, including CD4+ and $ү \delta T$ cells, may play an important role in early neutrophilic responses to silica exposure by IL-17A mediation [159]. Lastly, Van Berlo et al. demonstrated that alveolar epithelial cells also may contribute to inflammation following quartz exposure and these results were independent of NFKB driven mechanisms [160]. As previously mentioned, signaling by macrophages to different cell types is partially mediated by the upregulation of the inflammasome [155]. However, the 
secretion of mature IL-1 $1 \beta$ by activated macrophages in response to silica, which requires the transcription factor NF-kB [161], has been shown to be a two-step process based on reports from Beamer and Holian, as well as Cassel et al., suggesting that induction of the inflammasome by silica necessitates that macrophages first be primed by a stimulant, such as bacterial lipopolysaccharide (LPS) [123, 158]. Chen et al., have shown that silica itself can induce the activation of NF-KB, differentially from LPS [162]. Therefore, pro-IL-1 $\beta$ may be produced in response to this priming signal and later be cleaved when the inflammasome is upregulated by lysosomal damage, a DAMP resulting from the phagocytosis of silica as investigated by Hornung et al. and Hughes et al. [129, 163]. Moncao-Ribeiro et al. as well as Riteau et al. suggest that uptake of silica causes release of extracellular ATP succeeded by the activation of purinergic signaling that ultimately activates the NLRP3 inflammasome [87, 164]. Additionally, Peeters et al. demonstrated that the activation of the NLRP3 inflammasome by silica was related to particle surface reactivity, and moreover, that upregulation of this inflammatory pathway occurred in epithelial cells of the pulmonary tract in addition to myeloidderived cells [83]. Tsugita et al. have suggested that inflammasome activation may also be receptor signal dependent and that certain subsets of macrophages may contribute differently to acute versus chronic inflammatory processes [165]. Cassel et al. performed studies to address whether progression to silicosis is dependent upon inflammasome mediated IL-1 $\beta$ secretion, but not all inflammatory cytokines/chemokines secreted in response to silica related to this pathway [123], indicating the involvement of multiple pathways.

Other studies indicate that additional signaling mechanisms within the alveoli and associated tissue, particularly in the interstitium and lymphoid tissue, may play key roles in progression to chronic inflammation and fibrosis. Migliaccio et al. stated that alterations in APC phenotype and presentation may contribute to the initiation of chronic inflammation [92], though this is not necessarily independent from inflammasome activation. Other investigations led by Migliaccio et al point to a role for Th2 mediated immunity in development of silicosis as well [166]. While it is well-established that chronic inflammatory immune cell signaling is integral to the development of fibrosis, this is due in part to the release of fibrogenic factors by similar cells such as macrophages. Rom et al. demonstrated that macrophages exposed to silica signal for the proliferation of structural cells and their release of extracellular matrix proteins [167].

\section{4) Release of Fibrogenic Factors from Cells}

While uncontrolled inflammation has been stressed as a major factor in the development of fibrosis, it is likely not the only mechanism involved in the development of silicosis. Studies 
examining the role of immunosuppressive pathways in the development of fibrotic disease are being investigated. In work involving eicosanoids, Koren et al. highlighted the importance of silica modulation on the expression of eicosanoids and the opposing effects of lipoxygenase and cyclooxygenase (COX) enzyme pathways in macrophages following silica exposure. These experiments indicated that while the release of leukotrienes was increased, the expression of prostaglandin $(P G) E_{2}$ was reduced, suggesting the potential for increased leukocyte chemotaxis as well as fibroblast proliferation [168]. Studies conducted by Barbarin et al. recapitulated these findings and further elucidated that IL-10 influenced macrophages and lung fibroblasts to increase production of fibrogenic cytokines such as TGF- $\beta$, while down regulating COX-2, an important enzyme in the expression of an antifibrotic eicosanoid prostaglandin, $\mathrm{PGE}_{2}$ [169]. Other cell types have also been shown to be involved in the TGF- $\beta$-elicited effects during silicotic fibrosis. For example, Th17 cell activity has been implicated in many inflammatory diseases. Recently, Th17 cells were associated with epithelial-mesenchymal transition (EMT), an important process in embryonic development in normal tissue and also for cancer metastasis [170] and tissue fibrosis in pathogenesis [171]. Mi et al. demonstrated that IL-17A promoted the expression of EMT- related biomarkers in alveolar epithelial cells and that this was dependent upon TGF- $\beta$. Furthermore, this group established that IL-17A elicits the secretion of collagen by lung epithelial cells and that this too is dependent upon TGF- $\beta$ [172]. Therefore, the antiinflammatory cytokine TGF- $\beta$ is instrumental in these processes. Lo Re et al. evaluated the role of Tregs in expression of platelet-derived growth factor (PDGF) and found that this occurred through a TGF- $\beta$ mediated pathway and when these cells were transferred in vivo collagen deposition occurred. This group concluded that the inflammatory effects of T effector cells may lead to fibrosis in the absence of Tregs; however, Tregs play their own role in the fibroproliferative wound healing of the lung following silica exposure and both arms of the immune response need to be addressed to achieve effective therapy [173].

\section{Immune Effects}

There is a clear body of evidence suggesting the involvement of silica exposure and the development of autoimmune disorders, both from epidemiological data and in vivo study. The mechanisms by which this occurs have also yet to be fully elucidated. Most studies investigating this to date have been conducted ex vivo in samples from silicotic patients. Therefore, in vitro data is limited. It is likely that several pathways are involved as a vast diversity of autoimmune disorders are causally linked to silica exposure. Lee et al. hypothesize in their review that longterm stimulation of $\mathrm{T}$ effector cells and aberrant apoptotic response in contrast with increased 
apoptosis of Tregs is likely to be involved in this process [174]. In vitro evidence for this exists; it has been shown by Rao and Frey that silica exposure may cause altered APC presentation to lymphocytes that may result in their aberrant activation [175]. Additionally, Wu et al. examined the early activation marker CD69 on T cells exposed to silica and found that chronic activation of these T cells may lead to disruption of normal apoptotic mechanisms resulting in a chronically activated population of cells [176]. In their study examining the importance of apoptosis in selfantigen recognition, Pfau et al. concluded that silica exposure results in repeated apoptosis of cells that can increase the presentation of self-antigen and, therefore, autoantibody development [105]. Furthermore, Holian et al. showed that silica exposure resulted in a shift of macrophage phenotype to elicit more inflammatory conditions supporting the findings of Pfau et al. [177].

Other studies by Beamer and Holian suggest that the inhalation of silica might predispose individuals to infections due to alterations in APCs, disrupting their ability to adequately respond to pathogenic stimuli by downregulation of surface molecules and production of NO in response to bacteria [158]. Allison and Hart performed one of the earliest studies examining susceptibility to $M$. tuberculosis in macrophages. They determined that bacteria could more readily replicate and were released earlier from macrophages exposed to silica, and that this was dependent on very low dose exposure. These findings led them to hypothesize this as an explanation, in part, for susceptibility of silicotics to tuberculosis infections following chronic low dose exposure to silica [178].

The body of work mentioned above is only a summary of available literature comprising the current knowledge of silica exposure and the effects thereof. Though the field of silica exposure is widely examined, the mechanisms by which silica elicits toxicity, fibrosis, and susceptibility to infection are still not fully elucidated and require further investigation, especially when considering the potential for complications and alterations to these mechanisms in models of exposure to particle mixtures, which are prevalent in many current occupational platforms. Therefore, the current study is necessary to explore these effects further.

\section{Exposure to Diesel Exhaust (DE)}

Following the increased dependence on the combustion engine in the industrial era and the introduction of the diesel engine into the public-sector of transportation in the 1950s, hazardous health effects as a result of exposure to combustion exhausts have been extensively investigated. The association between pulmonary exposure to diesel and the development of 
diseases such as COPD, bronchitis, asthma, respiratory infection and cancer is well recognized $[5,6]$. The complex nature of diesel exhaust has resulted in variable methods of measuring and preventing exposure. Regulations exist for several common constituents of diesel exhaust including total particulate, total carbon (TC), elemental carbon (EC), carbon monoxide, and nitrogen oxides [179-183]. While the United States does have RELs in place for several constituents of diesel, the only regulations targeting diesel exhaust specifically use EC and TC, which encompasses DPM concentrations. The State of California Department of Health Services recommends an EC exposure limit of $20 \mu \mathrm{g} / \mathrm{m}^{3}$ [184], and the Mine Safety and Health Administration sets an exposure limit for $\mathrm{TC}$ at $160 \mu \mathrm{g} / \mathrm{m}^{3}$ in below-ground settings [182]. Furthermore, in addition to the known respiratory complications associated with diesel exposure, other systemic diseases related to these exposures have also been investigated, including cardiovascular and ischemic disease [185-192] and malignant diseases outside the lung including bladder cancer [193], prostate cancer [194], and ovarian cancer [195]. However, for the purposes of the current study, the review will focus on research related to the development of respiratory conditions as they relate to DE/DPM exposure, particularly as they relate to prolonged inflammation and alterations in immune function.

\section{Respiratory Effects of Work-Place Exposure to DE}

The results of epidemiological studies related to lung effects caused by occupational exposure to DE are variable and conflicting. The data available for these exposures are confounded by other respiratory exposures including cigarette smoke and inorganic dusts, particularly in the context of mining/tunneling. Additionally, the studies depend heavily on proxy interviews, and predominately examined risk for lung cancer only [196, 197]. Nevertheless, studies in the last few decades indicate DE exposure is involved in the development of not only malignancies, but also pulmonary diseases, and is particularly highly correlated with decreased lung function. Ulfvarson and Alexandersson conducted a study in stevedores utilizing diesel fueled trucks to load ships. The authors determined that the DPM component contributed to a reduction of lung function in workers exposed to total DE compared with groups exposed to particle-filtered DE. Further, it was concluded that declination in function reflected a restrictive pattern [198]. Chattopadhyay et al. showed DE exhaust exposure may lead to both restrictive and/or obstructive lung symptoms in their study investigating workers in a transit diesel bus garage. Data suggested that restrictive lung disease developments were dependent upon age, job function, and working tenure to some extent; additionally, it was suggested that current and previous smoking status played a role in these results [199]. Due to confounding exposures, 
there is very little epidemiological evidence to suggest that exposure to DE independently results in fibrosis or pneumoconiosis; although, some parameters such as radiographic findings have been examined. However, at very high levels of exposure, or in combination with other toxic respiratory exposure, outcomes of fibrosis may be plausible. In studies performed by Gamble et al. examining underground salt miners where diesel equipment was used, some evidence of pneumoconiosis was evident in a small number of cases; however, due to the small quantity of radiographic findings in these workers and the likelihood of confounding factors, these outcomes were not further investigated [200, 201]. Similarly, Gamble et al. examined respiratory symptoms in diesel-bus garage workers, and deemed radiographic evidence of pneumoconiosis to be insufficient for analysis and likely a result of confounding factors. However, it was found that a decrease in overall lung function occurred compared to other nondiesel exposed "blue-collar" workers; this result was associated with working tenure. Overall, smoking was the most significant risk factor indicated in this study [202].

Decrements in lung function due to DE exposure are more commonly associated with obstructive lung changes related to COPD, chronic bronchitis, and asthma. In an examination of workers who tested diesel engines at a manufacturing plant in China, Zhang et al. showed that exposure to DE led to lung function decrements, which were associated with long-term exposure. It was noted that these changes were most consistent with obstructive lung disease. Further, it was established that decreased lung function was not dependent upon smoking status [203]. Historically, COPD has been strongly linked with tobacco smoke and cigarette smoking in these studies. However, in addition to the Zhang et al. study, accumulating evidence suggests that exposure to DE specifically is linked with COPD in never-smokers. One medical surveillance study by Ulvestad et al. examined symptoms of COPD in tunnel workers (TW) compared with other heavy construction workers and determined that DE likely enhanced the risk for respiratory symptoms and COPD in TW [204]. An investigation of mortality in a cohort of construction workers by Bergdahl et al. led to the conclusion that fumes including DE may increase the risk of death by COPD particularly in never-smoking, construction workers [205]. In two retrospective cohorts examining railroad workers following the initiation of diesel locomotive use, Hart et al. determined that workers exposed to DE were at a higher risk for mortality caused by COPD than those who were not exposed to DE, and that this was dependent on amount of time worked, as well as job function. Further, while smoking status was positively associated with increased risk for COPD and tended to correlate with higher DE exposure, after controlling for this confounder, higher risk for mortality following DE exposure persisted, though it was slightly attenuated [206, 207]. In a population-based study examining 
cumulative lifetime occupational exposure and risk for COPD, Weinmann et al. found that those exposed to DE were at an increased risk for diagnosis with prevalent COPD regardless of smoking status [208].

Several studies have examined other obstructive respiratory symptoms associated with chronic upper airway inflammation or bronchitis. Jörgensen and Svensson found that among miners who worked below ground with diesel-powered vehicles, the frequency of bronchitis was increased and that this finding was dependent upon smoking status and age; however, no difference in lung function was noted [209]. Oliver et al. examined a cohort of Highway and Heavy $(\mathrm{HH})$ construction workers for lung function and respiratory symptoms. Authors concluded that only TW in the cohort were at an increased risk for chronic bronchitis. Additionally, it was found that risk for bronchitis increased with age and smoking status; conversely, working tenure was inversely associated with incidence of bronchitis. Workers who experience respiratory symptoms and/or are properly diagnosed by a physician may leave the trade contributing to a phenomenon known as the "healthy worker" effect. The authors suggest that this effect likely contributed to the finding that incidence of chronic bronchitis decreased as working tenure increased [210]. Gluck et al. investigated the role of chronic DE exposure on the nasal mucosa of customs officers who clear large diesel trucks. Their findings suggest that workers exposed to DE have increased hyperplasia of the nasal mucosa resulting from prolonged inflammation [211]. Finally, a study of toll-workers exposed to DE conducted by Safak et al. revealed that increased work duration resulted in increased bronchus wall thickening and decreased diameter of the large airways [212].

The incidence of asthma as a result of occupational DE exposure is now well-recognized and dates back to an initial case study by Wade and Newman in which they concluded that three railway workers developed asthma due to overexposure to $D E$, having no previous history of airway disease or exposure resulting in asthmatic symptoms [213]. Adewole et al. reported on three workers in diesel bus garages who developed asthma. It was concluded that the most probable cause of the asthma was chronic low-level exposure to DE with long periods of latency [214]. Furthermore, Oliver et al. reported an increased incidence of asthma in $\mathrm{HH}$ construction workers and TW exposed to DE, specifically in women, though this may partly be due to better reporting of symptoms to physicians by women. Additionally, it was established that incidence of asthma resulted in reduced lung function [210].

Research investigating the effects of DE exposure in the work-place has most historically been in an effort to determine the risk for developing lung cancer. Investigations on 
this subject vary widely in conclusion due to several confounding factors including the complex and variable properties of DE, potential co-exposure to other chemicals, particles and irritants, cigarette smoking, duration and magnitude of exposure, as well as age and other health complications. The findings relating to the development of lung cancer as a result of exposure to DE are summarized briefly (Table 2). Furthermore, based on this research and in vivo investigations, diesel exhaust was recently identified as a Group 1 carcinogen in the 2014 Monograph of the IARC [215]. The working group states that among the most convincing studies related to DE causality of lung cancer are the occupational cohort studies on railroad workers, miners, and transportation industry as a result of the clearly defined exposure levels and adjustment for confounding factors including cigarette smoking [216-219].

\section{Respiratory Effects of Environmental Exposures to DE}

In order to investigate the effects of inadvertent environmental exposure to DE, investigators have conducted studies examining traffic-related pollutants. Air pollution, and more specifically DE-related air pollutants, have been linked to reduced lung function, development of respiratory diseases, and malignancies. Brunekreef et al. examined school children who lived nearby to major roadways and measured pollutants in their schools. The authors of this study determined that decrements in the lung function of children were more closely associated with diesel-powered truck traffic, as compared to other motor vehicle exhaust (MVE) for which there was less correlation. Furthermore, the data suggested that this association was strongest in children who lived nearest to the motorways [220]. In a similar study, Northridge et al. examined students who attended a school in Harlem to assess whether bus depots placed in underprivileged areas of New York might contribute to the development of chronic lung disease. The authors suggest that adolescents in this region were subjected to above normal exposure to DE. Data gathered from this study also indicated that small airway obstruction, in the absence of symptoms, suggestive of asthma occurred in as many as $30 \%$ of participants. Furthermore, it was stated, since less than a third of those participants had ever been identified as having a history of asthma, that underdiagnosing and/or underreporting of asthma was likely occurring among Harlem adolescents [221]. Moreover, a cross-sectional study of asthmatic children living in close proximity to heavy traffic performed by Margolis et al. explained that distance of residence from highway was predictive of reductions in small distal airway size and risk for greater susceptibility to lung function decrements supporting the hypothesis that DE exposure may exacerbate existing asthma [222]. Further strengthening evidence for allergic disease outcomes is an epidemiological study that examined proximity of children to traffic-related air 
pollutants and the development of syndromes such as asthmatic bronchitis, hayfever, eczema, and allergic sensitization. Conclusions from this study demonstrated that children exposed to ambient particulate matter and living less than 50 meters from busy streets were at the greatest risk for atopic disease. Significant increased risk was found for bronchitis, hayfever, and pollen sensitization with relation to particulate matter with a diameter smaller than $2.5 \mu \mathrm{m}\left(\mathrm{PM}_{2.5}\right)$, and eczema was more closely associated with nitrogen dioxide $\left(\mathrm{NO}_{2}\right)$ exposure [223], both of which are components of DE.

In a population-based research study of traffic-related pollution conducted by Lindgren et al. it was found that residential traffic was associated with increased incidence of asthma and COPD diagnosis, as well as, symptoms associated with asthma and chronic bronchitis, suggesting that traffic-related pollution may have both short and long-term effects on human health [224]. A study by Beatty and Shimshack analyzed the ability of filters to reduce school bus exhaust emissions within residential communities and their impact on the health of adults and children living nearby. Overall, it was found that incidence of asthma, bronchitis, and pneumonia were reduced in both children and adults with chronic respiratory symptoms and numbers of hospital cases for these complaints were reduced after retrofitting the school buses to reduce DE [225]. Salameh et al. concluded that outdoor air pollution caused by both heavy traffic or residing in proximity to a power plant may increase incidence of chronic bronchitis, though home heating methods and passive smoking were also found to be factors [226]. In a study by Shultz et al. the associations between the development of asthma, allergies, and wheezing were investigated in relation to either annual $\mathrm{PM}_{2.5}$ exposure, industry related exposure, or traffic exhaust exposure. The data demonstrated results similar to studies described above; incidence of allergic asthma was associated with increasing exposure to particulate matter, and proximity to roadways had the largest increase in asthma that suggests traffic-related $\mathrm{PM}_{2.5}$ may be a significant risk factor for developing asthma [227].

\section{Environmental Exposure to $D E$ and Susceptibility to Infection}

Evidence from epidemiological observations also suggest that susceptibility to infections in the respiratory tract may be increased following environmental exposures to DE. Increases in some components of $\mathrm{DE}$, including $\mathrm{NO}_{2}$ and $\mathrm{PM}_{10}$, and ozone $\left(\mathrm{O}_{3}\right)$, an allotrope of oxygen that is created as a result of chemical interactions between combustion products $\left(\mathrm{NO}_{2}\right.$ and volatile organic compounds (VOCs)) and sunlight, were shown to be associated with increased hospital admissions for pneumonia and influenza [228]. A population-based study by McGowan et al suggested that increases in $\mathrm{PM}_{10}$ in the environment resulted in increased hospital admissions 
for pulmonary infections including pneumonia, influenza and other acute respiratory infections. Furthermore, they explained that while admissions across age groups were similar, children were at a slightly higher risk for hospitalization [229]. Neupane et al. revealed that older adults exposed to higher levels of air-pollution including components of DE, including $\mathrm{PM}_{2.5}$ and $\mathrm{NO}_{2}$ were at an increased risk for hospitalization for pneumonia [230].

\section{Experimental Exposures to DE in Human Volunteers}

\section{Pulmonary Effects}

In an effort to determine the risk posed to humans by DE exposure by eliminating confounders that are present in epidemiological and occupational assessments, several researchers have conducted experiments in human volunteers in controlled environments. Many of these studies examine a particular component of DE in order to determine which properties have a causal relationship with the health effects that have been observed in epidemiological studies. A study by Rudell et al. used particle trap filters to reduce DPM when exposing healthy human subjects to DE. Additionally, they sought to determine what effects DE had on cells recovered from bronchoalveolar lavage. Findings from this investigation resulted in the conclusion that DE exposure caused inflammatory cell influx into the airways and that trapping particulate did not significantly reduce these effects, suggesting that gaseous components might be partially responsible for these effects [231]. However, in a separate study, Rudell et al. examined additional methods of filtration in the cabins of diesel automobiles and evaluated their effects on vehicle occupants following exposure. Particle-only filtration did not reduce symptoms experienced, though particle filters in combination with active charcoal filtration did significantly reduce reported symptoms. This is attributed to the reduction in the hydrocarbon components of the gaseous fraction of DE. It should be noted that reduction of symptoms with filtration was based on self-reporting by subjects. No measured parameters of lung function or nasal lavage were altered between exposure filtration variations or controls [232]. It is also important to note, with regard to self-reporting, that a separate study conducted by Carlsten et al. demonstrated that perceived exposure to DE by study subjects in a blinded exposure study was more important in prediction of symptom reporting than DE exposure itself, and therefore, symptom-independent studies may be more useful in determining effects of DE [233]. Conversely to findings relating to DPM by Rudell et al., are the conclusions of a study by Nightingale et al. that investigated whether or not DPM caused inflammatory response in the airways of healthy volunteers. While they did not observe any changes in lung function or 
cardiovascular parameters measured in this study, authors did observe airway inflammation at high concentrations of DPM [234], a conclusion not based on self-reported symptoms.

In order to more fully elucidate the impact of DE on the airways, Salvi et al. exposed healthy volunteers to dilute DE during intermittent exercise. The authors of this study reported that acute short-term exposure to DE caused systemic and pulmonary inflammation demonstrated by elevated neutrophils recovered in peripheral blood and lavage fluid, respectively [235]. Behndig et al. exposed healthy volunteers to DE to determine if pulmonary cells would elicit an antioxidant response to low doses of DE, similarly to results observed in in vitro studies. Though they did observe inflammation and antioxidant responses in response to DE exposure, it was in the conducting bronchial airway and not in the alveolar region suggesting that pulmonary response to DE was compartmentalized [236]. In an investigation of DE exposure on airway inflammation, Sehlstedt et al. aimed to clarify whether engine load or transient speed altered effects observed with DE generated by idling engines. Authors explained that DE produced by urban engine cycling increased the expression of adhesion molecules in bronchial vascular endothelial cells and resulted in bronchoalveolar eosinophilia, a finding not previously reported in relation to responses to DE generated from idling engines. These results support the idea of variable health outcomes in response to DE produced under differing conditions [237].

Furthermore, the composition of DE is complicated by the interaction of its components with properties of the environment such as water vapor and sunlight. As previously mentioned, when some combustion components chemically react with the environment, additional hazardous inhalants such as $\mathrm{O}_{3}$ may be formed. Therefore, some experiments have been performed to determine whether these reactions may contribute to health effects observed for environmental exposures to DE. A non-invasive technique for the detection of airway inflammation following controlled exposures that measures fraction of exhaled nitric oxide (FENO) as the injury parameter has been used as a marker of adverse effects in the lung. In a study conducted by Barath et al. DE was shown to enhance FENO and that this effect was localized to the central airways; however, this technique was not effective for detecting the inflammation caused by $\mathrm{O}_{3}$, possibly due to the varying mechanisms by which $\mathrm{DE}$ or $\mathrm{O}_{3}$ induce inflammation [238]. An investigation carried out by Bosson et al. showed that $\mathrm{O}_{3}$ exposure subsequent to DE had the ability to enhance DE-related airway inflammation, highlighting the complexity of additional influences when considering health effects caused by exposure to environmental pollutants [239]. A study by Madden et al. investigated the combinatorial effects 
of $\mathrm{DE}$ and $\mathrm{O}_{3}$ on lung function, both on healthy volunteers, as well as subjects with the gluatathione S-transferase-Mu1 (GSTM1) genotype. GST enzymes have been shown to mediate damage caused by air pollution health effects by detoxifying products of oxidative stress and individuals with this phenotype have been shown to be more susceptible to inflammatory diseases [240]. Madden et al. confirmed the effect of environmental mixtures demonstrated by Bosson et al. In their study, $\mathrm{O}_{3}$ caused a decrement in lung function, and although $\mathrm{DE}$ did not mirror this effect on its own, in combination with $\mathrm{O}_{3}$ it led to greater decreased lung function. However, this effect was not observed for GSTM1 individuals suggesting that oxidative stress may not be involved in decreased lung function [241].

Based on epidemiological findings suggesting that DE may exacerbate pre-existing conditions of allergies and asthma [242], several studies have conducted controlled human DE exposure in asthmatics and allergic rhinitics. Larsson et al. exposed allergic rhinitics to $D E$, at doses known to cause inflammation in healthy individuals, in an effort to determine whether $D E$ exposure enhanced inflammation in the lower airways of individuals with pre-existing allergies. No neutrophilic inflammation in the lower airways of participants was detected. However, evidence of possible mast cell degranulation was found [243]. In a study carried out by Stenfors et al., asthmatics were compared to healthy subjects in an exposure study investigating whether $\mathrm{DE} \mathrm{PM}_{10}$ may exacerbate existing allergic responses in asthmatics. Similar to the study by Larsson et al., DE exposure did not enhance neutrophilic inflammation in asthmatics. This is in contrast to the study that previously demonstrated healthy subjects did experience airway inflammation [244]. Another study by Behndig et al. also examined DE exposure on asthmatics comparing results to healthy controls. While they found that $D E$ induced neutrophilic inflammation in healthy subjects, this was not the case for asthmatics leading to the conclusion that neutrophilic inflammation and classical cellular indicators of allergic airway response are not necessarily accurate measure of allergic airway response to traffic-related pollutants [245]. Findings from these experiments demonstrate the complicated nature of exposure-related health effects, particularly in the context of complex particle mixtures and the need for further studies directly examining mechanisms for pathological development.

In order to more fully understand the response of asthmatics to environmental pollution in an environmental setting, McCreanor et al. exposed asthmatics to real-time traffic exhaust. The authors reported that those participants exposed to higher amounts of ultrafine particles and EC from diesel-powered traffic developed increased sputum myeloperoxidase, an indicator of neutrophilic inflammation, and greater reductions in lung function. These results were 
exacerbated for individuals with more severe pre-existing asthma. The data from this real-time exposure study are more consistent with epidemiological data suggesting that laboratorycontrolled measures may not accurately represent physiological exposures in the environment [246]. Additionally, this study highlights the importance of designing studies aimed at examining complex exposures modelling realistic exposure scenarios to the greatest degree possible. This was corroborated in a study by Hosseini et al. demonstrating that effects of DE exposure may be overlooked by administering exposures in a sterile, uncomplicated environment, such as a laboratory. Hosseini et al. sought to elucidate whether DE may be acting as an adjuvant in combination with allergen-induced asthma and airway inflammation. These authors found minor or nonexistent changes in eosinophilia and mast cells of the airways; however, other sensitive measures of allergic response including CD4, IL-4, neutrophil elastase, and CD138, were increased. Moreover, these parameters of allergic response were not elevated for either DE or allergen exposure individually, but were synergistic in combination. This study further highlights the important interactions of the environment with pollutants, which may affect health effects [247].

\section{Susceptibility to Infection}

In addition to the studies of pulmonary effects following controlled exposures in human subjects discussed above, studies investigating epidemiological findings that support the hypothesis that DE may lead to enhanced susceptibility to infection have also been conducted. Due to ethical ramifications of directly infecting individuals with pathogens, there are a limited number of these controlled exposure investigations. Additionally, many of these studies use a two-step approach with the toxicological exposure performed in vivo, while the investigations on susceptibility are conducted ex vivo using cellular samples collected following the exposure, which should be taken into consideration when interpreting the data. In a study conducted by Frampton et al., it was shown that macrophages obtained from humans exposed to the DEcomponent $\mathrm{NO}_{2}$ were less effective in inactivating influenza virus ex vivo [248]. Another recent study indicated that DPM reduced natural killer (NK) cell recruitment and activation during influenza infection in allergic rhinitics following exposure to particles. Further, it was demonstrated that during the context of viral infection in allergic rhinitics, allergic inflammation may be worsened due to an inability or decreased ability for NK cells to target and clear eosinophils [249]. Helleday et al. examined the mucociliary function of bronchial cells in human volunteers following short-term exposures to $\mathrm{NO}_{2}$ as a measure of effects on mechanical barriers to infection. The authors concluded that pollutant exposure caused significant reduction 
in the mucociliary activity of these cells, but that the effect was reversible [250]. Though $\mathrm{NO}_{2}$ is a known component of DE, studies of this nature have not examined DE itself as a whole, and therefore, results relating to whole DE may vary from these depending on the composition of DE with relation to $\mathrm{NO}_{2}$.

\section{Effects of Respiratory Exposure to Diesel Exhaust in in vivo Animal Models}

\section{Pulmonary Effects}

While controlled studies in humans are valuable in determining physiologically relevant health effects that may be caused by exposure to DE, ethical restraints and limited participation significantly restricts mechanistic determinations of disease etiology. Therefore, equally important are studies using laboratory animal models of exposure. As in epidemiological studies, studies evaluating systemic effects of DE exposure, particularly related to cardiovascular disease have been conducted [251-257]; however, the review below will focus on the animal studies related to respiratory effects specifically.

Chen et al. examined species-specific responses in rats and guinea pigs to DE and demonstrated that DE, while not necessarily cytotoxic to pulmonary macrophages studied ex vivo following exposure, did demonstrate reductions in phagocytosis despite sufficient adherence and motility. [258]. This study provided some of the first evidence that DE may reduce function in AMs. Another study by Chen et al. examined the species-specific effects of DE in a one-year, chronic, time-course study. Data indicated that biological responses were dose and duration dependent. [259]. A study by Pepelko et al. conducted in cats suggested that minor changes in some lung function measurements may occur after 28 days of exposure to $\mathrm{DE}$, but data was not suggestive of significant lung damage for the dose and duration examined [260]. Abraham et al. examined lung function in sheep following exposure to DPM but did not find significant alterations in pulmonary resistance or airway reactivity, though exposures were acute [261]. Gross assessed the effect of chronic exposure to DE on the pulmonary function of rats. Similarly, no significant changes were observed following approximately 9 months of DE exposure [262]. A continued study by Gross examining function in rats after 2 years of continuous exposure to DE suggested some significant alterations in lung function including changes in vital capacity and forced expiratory volume (FEV) [263]. Patterns of lung function reductions consistent with restrictive changes were found by Moorman et al. in their study, though amounts of DE were high compared to other studies, and they used a cat model [264]. In a study of chronic DE toxicity conducted in three species, Heinrich et al. determined that 
chronic exposure to unfiltered DE caused significant changes in lung function and alveolar clearance primarily in rats as compared with mice and hamsters. Additionally, tumors developed in mice in response to both unfiltered and filtered DE; whereas, only unfiltered DE resulted in lung tumor incidence in rats. This study further outlines the importance of animal model choice in demonstrating relevant health effects of exposure to DE [265]. Moreover, in a study comparing exposure of rats and mice to dilute DE, Henderson et al. established that despite mice having a higher soot burden in the lung, rats exhibited more fibrogenesis. The authors determined that this was related to the propensity of rat alveolar cells to release greater amounts of inflammatory mediators including leukotriene B4 and PGF2 alpha than mouse cells [266]. Finally, in a study by Ulfvarson et al. examining effect of DPM trapping on changes in respiratory effects on rabbits it was established that the gaseous components of DE may result in reduced compliance of the lung suggestive of fibrotic changes [267]. It is important to note that, in addition to species-specific effects, the studies discussed above employ different doses and durations of exposure, which may also account for differences observed in the study outcomes. Therefore, model should be chosen based upon which health outcome is being examined. While rats demonstrate similar inflammatory response and histological changes observed in humans, cats may reflect more consistent functional changes, and guinea pigs allergic developments, which are more similar to epidemiological findings.

Beyond characterizing overall pulmonary toxicity caused by DE in animal models, investigations have aimed to determine more specific mechanisms for outcomes observed in humans. Inflammation, specifically, has been widely investigated as the initiating event leading to different pulmonary diseases caused by inhalation of DE through a variety of pathways. Inoue et al. examined the role of toll-like receptor (TLR-4) in inflammation caused by DPM. By comparing responses to IT administration of DPM, at a dose of $12 \mathrm{mg} / \mathrm{kg}$, in TLR-4 mutant mice to $\mathrm{WT}$, the authors discovered alterations in the expression of inflammatory mediators of chemotaxis, suggesting that DPM signals in part through the TLR-4 pathway in order to induce inflammation [268]. Fujimaki et al. showed that IL-6 was essential for the induction of airway inflammation mediated by cellular influx and the expression of chemotactic factors. The authors determined this by comparing DE inhalation exposure; either 1 or $3 \mathrm{mg} / \mathrm{m}^{3}$ for 12 hours per day for 4 weeks, in WT and IL-6 deficient mouse strains [269]. Ahn et al. investigated the role of COX-2 in inflammation induced by DPM. In this study, the authors exposed mice oropharyngeally to 1,10 , or $25 \mathrm{mg} / \mathrm{kg}$ of DPM per day for five consecutive days and showed that DPM exposure caused inflammation that is mediated by COX-2 by ameliorating these effects with an anti-inflammatory treatment with glucocorticoid, dexamethasone [270]. 
Oxidative stress, as discussed at length with regard to inflammation caused by silica exposure, has been indicated as a potential initiator of lung injury caused by DE as well. Sagai et al. examined the possibility that DPM may produce biologically active ROS in the lung following IT exposure of mice to varying doses of DPM between $0.4 \mathrm{mg}$ and $1.0 \mathrm{mg}$. Data from their experiments showed that DPM-exposed mice had increased mortality due to oxidant radical-mediated endothelial damage resulting in pulmonary edema [271]. Lim et al. demonstrated that either 100 or $200 \mu \mathrm{g}$ of DPM, administered in repeated IT, 1 time per week for 10 weeks, elicits airway hyperresponsiveness mediated by inflammation caused by superoxide (SO) and NO. They showed that DPM increased the activity of NO synthases (NOS) and decreased superoxide dismutase (SOD) activity. Further, treatment with inhibitors of NOS reduced inflammatory effects of DPM treatment [272]. Ito et al. found that in rats exposed to $2 \mathrm{mg} / 0.5 \mathrm{ml} / \mathrm{kg}$ of DPM intratracheally, alveolar cells infiltrating the lung were nearly doubled compared to controls, that peroxynitrite formation was increased, and that levels of inducible NOS (iNOS) mRNA were enhanced, suggesting that DPM caused oxidative-induced inflammation in the lung [273]. Han et al. used noninvasive spectroscopy in conjunction with a membrane-impermeable nitroxyl probe to detect the generation of hydroxyl $(\mathrm{OH})$ radicals following exposure to DPM in mice. When scavengers of radicals were applied, the rate of decay of the probe was decreased. The study provides further evidence that DPM causes increased production of ROS that may contribute to lung injury following exposure [274]. Finally, Nemmar et al. aimed to determine if Emodin, an antioxidant, may reduce DPM-induced inflammation and oxidative stress, which contribute to decrements in lung function following DE exposure. In their findings, it was reported that treatment with Emodin reduced airway resistance, neutrophilic inflammation, and production of inflammatory cytokines. The authors concluded that anti-oxidant treatments like Emodin may be useful for protection against pollutant-derived lung toxicity [275].

An important factor in toxicity of DE is the composition of the material. DE composition can vary based on a number of factors including fuel source and engine type as discussed previously. Similar to studies with human volunteers, investigators have sought to determine which component(s) of DE may be contributing to oxidative stress. Madden et al. explored the possibility that, similar to effects observed in humans, $\mathrm{O}_{3}$ may cause exacerbation of DPMrelated toxicity. Treatment of DPM particles with $\mathrm{O}_{3}$ prior to administration in rats resulted in enhanced inflammatory and injurious responses in the lung. These effects were not observed with the control particle, CB (a core component of DPM), suggesting that this effect was related to interactions of $\mathrm{O}_{3}$ with components adsorbed to DPM particles [276]. Investigations by Zhao 
et al. examined the effect of DPM or the organic extract of DPM (DPME), which lack the carbon particulate core, on parameters of toxicity. In one study, they examined blockade of iNOS and cytochrome p450 (CYP)-1A1 in mediating oxidative stress caused by AMs. It was highlighted that NO played a role in inflammation caused by the organic fraction of DE and expression of CYP1A1 by both the particulate and organic fractions of DE, but that NO regulation does not affect inflammation induced by DPM [277]. In their subsequent study, Zhao et al. examined oxidative stress in both WT and iNOS knockout mice. Their results demonstrated that inflammation initiated by DPM was mediated by AM production of ROS and NO. Lack of NO production had little effect on DPM induced oxidative stress. NO was also important for initial induction of neutrophilic inflammation, pulmonary capillary damage, and IL-12 production. Further, since DPM and carbon black (CB) induced inflammatory responses and injury, but the DPME did not, these results were attributed to the particulate core and not the substances adsorbed to it [278].

As epidemiology studies and in vivo studies indicate, obstructive lung diseases including COPD and bronchitis are commonly associated with DE exposure. In vivo studies have been conducted to determine the potential etiological mechanisms. A study by Fedan et al. indicated that chronic inhalation exposure of rats to $2 \mathrm{mg} / \mathrm{m}^{3}$ of DE 7 hours per day, 5 days per week, for two consecutive years, resulted in alterations of airway smooth muscle functions that may contribute to airway obstruction [279]. A chronic exposure study, conducted by Ishihara et al., over a 24-month time-course evaluating rats exposed to DE, determined that moderate to high levels of DE exposure (corresponding to between 1-3 $\mathrm{mg} / \mathrm{m}^{3}$ particles at 18 months) resulted in chronic inflammation, increased mucus and phospholipid production consistent with the development of COPD. Moreover, these responses occurred in a time and concentration dependent manner; though, some parameters plateau just after a year of exposure [280]. To further understand which components of DE may be associated with obstructive lung disease, Laks et al. treated DPM by different extraction processes and assessed whether exposure to the extractions altered airway and lung mechanics following intransal exposure to $15 \mu \mathrm{g}$ of DPM in mice. Treatment with hexane was the most effective in removing measured polycyclic aromatic hydrocarbons (PAHs), common components adsorbed to the carbon core of diesel particles. Data suggested that PAHs may be important for pulmonary health effects observed based on the findings that hexane treatment reduced alterations in lung parenchyma and lung mechanics observed with untreated DPM [281]. Experiments examining airway inflammation in response to repeated exposure DPM, the particulate fraction of DE, intranasally instilled in mice were conducted by Yoshizaki et al. who demonstrated that exposure to $30 \mu \mathrm{g}$ DPM 5 days per 
week for 60 days resulted in increased mucus production and nasal epithelium thickness, indicating that DPM treatment resulted in respiratory tract inflammation [282]. A study by Shaheen et al. aimed to understand the role of ceramide in airway inflammation following exposures to DE at concentrations below those required to initiate biomarkers of oxidative stress and inflammation. Cell stressors can induce ceramide regulated NF-KB initiation and apoptosis. Inhibiting ceramide following exposure to DE, reduced not only ceramide but also increased expression of enzymes involved in sequestration of ceramide-related precursors. Overall, data suggested that even in the absence of measurable inflammation as a result of DE exposure, biomarkers related to inflammatory initiation are up-regulated, demonstrating more sensitive measures for low-level exposure health effects [283]. Overall, in vivo studies have demonstrated that subacute and chronic exposures to DE resulted in enhanced airway inflammation, increases in mucus production, and airway changes consistent with obstructive pathologies such as COPD; moreover, this may be related to the presence of PAHs in DE since some of these effects were attenuated when PAHs were reduced.

The development of AHR, asthma, and allergic asthma are the most well-investigated respiratory diseases relating to $D E$ exposure. DE has not only been shown to result in the presence of asthma de novo, acting as an allergic adjuvant, but also to exacerbate the clinical presentation of pre-existing disease following exposure in humans [213, 214, 227, 242, 246]. Therefore, extensive investigations have been made into discovering the biological mechanisms that promote this pathology. Several studies have examined the role of oxidants in development of asthma following DPM exposure. Sagai et al. examined the role of oxidant inflammation on airway reactivity. In one study, it was shown that DPM reduced scavenging of oxygen species in the airways resulting in increased $\mathrm{NO}_{2}$ and enhanced AHR [284]. In a subsequent study, Sagai et al. determined that DPM caused increased inflammation, proliferation of mucus producing cells, secretion of mucus, respiratory resistance and constriction of airways. Additionally, eosinophilic infiltration was exacerbated. These parameters were abrogated by pre-treating with polyethyleneglycol-conjugated SOD (PEG-SOD) to quench oxidants, suggesting that oxidants derived from DPM exposure resulted in symptoms consistent with asthma [285]. A group led by Aguilera-Aguirre showed that mitochondrial dysfunction resulting from oxidative stress was responsible for exacerbating allergic inflammatory response. This finding suggests that DE exposure leads to production of oxidants that stimulate further cellular ROS and induce dysfunction of mitochondria in airway epithelium and in turn may be responsible for induction of airway inflammation [286]. 
Though increased oxidant burden may result in increased parameters of inflammation resulting in asthmatic disease, it may also stimulate the release of proteins from immune cells, which may contribute to mechanisms of pathology. Ohta et al. conducted a study to further elucidate the role of cytokines in response to DPM exposure in the development of bronchoconstriction and airway hyperresponsiveness. It was demonstrated that granulocyte macrophage colony stimulating factor (GM-CSF) was intrinsic to the development of airway hyperresponsiveness to DPM. Blockade of GM-CSF resulted in reduction of bronchoconstriction, and hyperplasia of clara cells, both hallmarks of asthma. While IL-4 also contributed to these effects, it was to a lesser extent [287]. A related study led by Yamashita established that PDGF contributes to the remodeling of the airway following DPM exposure. Although PDGF antibody treatment did not alter influx of inflammatory cells into the airways, it reduced airway wall thickening elicited by DPM. These results suggest that PDGF, a regulator of fibroblast growth and proliferation, played a distinct role in collagen modulation and thickening of the asthmatic airways following exposure to particle [288]. More recently, IL-17 has been implicated in the pathogenesis of inflammatory diseases. Brandt et al. sought to establish whether DPM exposure exacerbates allergic asthma via an IL-17 mediated response. Results from this study suggest that Th17 cells contribute to DPM-mediated allergic asthma. Antibody neutralization of IL-17 resulted in abrogation of the enhanced AHR caused by DPM in this model. Furthermore, the authors examined children with pre-existing allergic asthma in order to determine their serum levels of circulating IL-17 based on estimated DPM exposure. They report that children with high estimated exposures have levels nearly 6 times higher than children exposed to low levels of DPM and that they reported more frequent and severe symptoms [289].

Others have examined the role of DPM exposure in conjunction with allergen to elicit airway responsiveness. A study by Suzuki et al. indicated that DPM and pyrene, a PAH associated with DPM, act as adjuvants and stimulate greater production of immunoglobulins when given in co-exposure with house dust mite (HDM) allergens [290]. Increased antibodies to allergens may ultimately result in incidence of asthmatic symptoms. Investigation by Takano et al. corroborated these results with a different allergen. The authors of this study reported that DPM in combination with ovalbumin (OVA) resulted in increases of parameters of allergic asthma including increased eosinophilic inflammation, goblet cells, and expression of Th2 cytokines IL-4 and IL-5. Further, they demonstrated enhanced levels of immunoglobulin (Ig) G and IgE. Results indicated exacerbation of allergic asthma when compared with DPM or allergen alone [291]. Miyabara et al. established that DE caused enhanced eosinophilic and 
neutrophilic infiltration, expansion of goblet cells increases in IL-5, IgG, and AHR in combination with OVA sensitization. An increase in IgE was not observed, but authors speculate this may have been due to the difference in route of administration. Inhalation was used in this study, whereas, in previous studies instillation by nasal route was used. It is possible that sensitization by direct contact with the nasal epithelium might result in increases observed in $\lg E$ observed previously [292]. Results have been reviewed for two types of allergen models, HDM and OVA, but another group sought to determine whether DPM would enhance allergic response to pollen as well. Steerenberg et al. determined that, as demonstrated in previous allergen studies, DPM enhance allergic response to pollen. This study further demonstrated the adjuvant effect of DPM on allergy and asthma [293]. An investigation by Hao et al. sought to determine what acute effects DPM may have on airways in the absence of their adjuvant allergic influences. The authors established that even in the absence of allergen, DPM exposure increased airway reactivity suggesting that not only does DPM elicit/exacerbate allergic asthma but that it may directly induce acute asthmatic symptoms associated with AHR [294].

Many studies have been conducted to try to determine what component of DE and/or DPM are correlated to pathogenesis. The composition of DE/DPM is widely variable as previously discussed and this tenet appears to govern the course of pathologies associated with exposure. Variable responses to DE/DPM reported epidemiologically and based on research investigations appear to be directly related to the characteristics of the particles used to conduct studies, and those conducted in vivo are briefly summarized below. An investigation by AlHumadi et al. demonstrated that the adjuvant effect of DPM on the development of allergic airway disease may not be dependent on particle composition. By comparing DPM combined with OVA sensitization with CB combined with OVA sensitization as a particle comparison control, the authors showed that both co-exposures elicited similar increases in OVA specific $\lg G$ and $\lg E$, suggesting that both augmented allergic sensitization [295]. Inoue et al. aimed to determine which components of DPM might be responsible for observed toxicities. This group examined extracts of DPM organic components and washed DPM carbon nuclei. It was explained by the authors that in response to co-exposure to OVA antigen organic components did not elicit responses that were significantly different than the OVA control group; whereas, washed DPM nuclei had the greatest impact on induction of airway hyperreactivity (AHR). These results point to the carbon nuclei as being important in airway inflammation in the context of allergic asthma [296]. As previously mentioned in a study by Laks et al. it was found that the PAHs associated with DPM contributed to pulmonary toxicity [281]. A third study by Park et al. investigating the components of DE and their importance in the context of inflammation found 
that how DPM are recognized by phagocytic cells, determines the inflammatory effect that they will induce. They explain that DPM, which are engulfed by cells result in a Th2-type response; whereas, DPM, which are not taken up by cells induce a Th1-type response. Furthermore, they suggest that the physicochemical properties like size and charge determine how well they are able to be taken up and how well they elicit stress responses like oxidation that has been associated with both Th1 and Th2-types of inflammation caused by DPM [297]. A study by Tanaka et al. determined that the gaseous components of DE may be most important for the Th2 polarizing skew of DE, as exposure to DPM combined with OVA in the absence of gaseous components failed to enhance eosinophilic inflammation or Th2 cytokines to the level of DE without particles [298].

Though $\lg E$ is well-established in the importance of allergic development, IL-4 upstream of $\mathrm{IgE}$ is also critical as is the polarization of the immune system to a Th2-mediated response to stimulus that acts as a signal to initiate immunoglobulin class switching [299]. Experiments conducted by Jang et al. aimed to elucidate whether $\mathrm{O}_{3}$ in combination with DPM might lead to increased presence of asthmatic symptoms. These studies established that, similar to studies conducted in human volunteers, $\mathrm{O}_{3}$ may exacerbate the induction of asthma observed following exposure to DPM and allergen. Increases in pathological response were additive and related to enhanced levels of IL-4 and AHR following co-exposure to DPM and $\mathrm{O}_{3}$ in an allergen sensitized mouse [300]. More recent experiments relating to $T$ cell differentiation following exposure to DPM were performed by Liu et al. in a study of DPM effects on DNA methylation. These authors demonstrated that combined exposure to DPM and fungal allergen resulted in increased methylation of IFN-y and decreased methylation of IL-4. Additionally, IgE production was enhanced following exposure to both DPM and fungal allergen. These results suggest that DPM exposure altered differentiation/function of immune cells in the presence of allergen by altering DNA state, potentially leading to a Th2 helper polarization and allergic asthma [301]. Finally, in a recent study of DPM, De Grove et al. investigated the role of newly discovered innate lymphoid type 2 cells (ILC2) in allergic asthma elicited by DPM. They compared responses of WT mice with either ILC2 deficient, lymphocyte deficient, or Gata 3 insufficient mice following exposure to DPM in combination with HDM allergen. The co-exposure resulted in AHR and inflammation in WT mice and this was reduced in either Gata 3 insufficient and marginally so in ILC2 deficient animals. In lymphocyte deficient mice, it was abolished entirely suggesting that intact adaptive responses are required for an adjuvant effect by DPM to be provoked [302]. 
Extensive research has also been conducted in an effort to understand whether and by what mechanism DE initiates tumorigenesis in the lung. These studies date back to the 1950s to a study by Kotin et al. that determined that PAH components of exhaust resulted in tumors when painted onto the skin of mice [303, 304]. The validity of many of the respiratory toxicity studies in animals have created controversy due to evidence that species such as rats may reach a burden of particle considered to be "overload" in which the mechanisms of clearance are overwhelmed resulting in neoplasms [75], but that this bears little on the toxic properties of the particles themselves due to composition. Further these studies were often conducted at doses unrealistically higher than correlated to physiologically relevant levels of exposure in humans, and due to more superior clearance mechanisms it was suggested to be unlikely for humans to reach overload, and therefore carcinogenesis was not considered to be a valid detriment posed by DE exposure [305, 306].

In their very thorough review of $D E$ cancer effects, Hesterberg et al. weighed the advantages and disadvantages of traditional (old technology emission prior to 2000) DE exposure research to address that point. Study design, dose, route of exposure, models, methods, and composition of the particles are all among the examined variables relating to research findings on DE tumorigenesis. Ultimately, the authors do conclude that the only consistent findings for carcinogenicity in laboratory animals are in rats and do not provide sufficient or convincing evidence that these results translate to humans, as it appears to be a species-specific phenomenon. However, they do allow that new technology (since 2000) DE (NTDE) exposure risks be assessed separately as the chemical composition of new emissions renders it completely different chemically from traditional DE [306], whereby the particulate component is significantly lower, but other gaseous components may not be.

Additional investigations into both traditional and NTDE exposure and the potential for carcinogenesis have been conducted. Moreover, research has also been conducted in animals evaluating levels of $D E$ exposure or exposure to specific components of $D E$, which are far lower than what would be considered "overload" but that still show evidence for carcinogenesis. Further, as of 2012, DE was reclassified by IARC as a Group 1 carcinogen, carcinogenic to humans, 'having sufficient evidence' in humans for the carcinogenicity of diesel engine exhaust. Additionally, in the 2014 Monograph it is also stated that there is 'sufficient evidence in experimental animals' for the carcinogenicity of whole diesel engine exhaust and diesel engine particulate matter, but 'inadequate evidence' in the gas-phase of diesel engine exhaust [215]. 
Therefore, only a brief summary of studies employing whole DE or DPM will be summarized here.

Heinrich et al. found that exposure of rats to diesel soot increased incidence of lung tumors in rats, but no significance for lung tumors was indicated in mice [307]. In a study conducted in rats by Mauderly et al. it was determined that DE and CB particles produced similar carcinogenic effects. Their findings suggest that small, carbonaceous particles, in the respirable range are responsible for lung cancer development at high concentrations, and that constituents of DE otherwise determined to be mutagenic play a lesser role in tumor developments in rats. Though they report the biological mechanism to be unknown as of yet, it does correlate to impaired particle clearance and progressive particle accumulation resulting in increased particle load [308]. An investigation by Nikula et al. examining rats also showed increased neoplasms, both malignant and benign, following exposure to DE or CB. Additionally, they observed increased incidence in females when compared with males, and in males the incidences were slightly higher for $D E$ than for $C B$, though this may have been due to an enhanced mortality that occurred in male rats exposed to CB. Overall, the particulate component of DE was determined to be the more critical portion for carcinogenic effects [309]. A study by Iwai et al. showed more mechanistic results explaining that tumor formation in the lungs of rats following a long latent period subsequent to exposure was due to oxidative stress on DNA induced in early phases of exposure to DE [310]. Stinn et al. also reported that DE particle deposition and accumulation led to tumorigenesis in rats exposed to DE; however, they report that this happened in the absence of any significantly noted DNA adduct formation or cell proliferation, but rather correlated to a dose related increase in sustained inflammation [311]. Ultimately, in animals, evidence of mutagenicity is limited and mostly observed in rats. However, evidence suggests that DE results in impaired clearance mechanisms, which requires further investigation, particularly at low levels of exposure.

\section{Immunological Effects and Susceptibility to Infection}

Human-controlled experiments in addition to epidemiological evidence suggest that exposure to DE results in increased susceptibility to infection, particularly in the respiratory tract. Therefore, in vivo animal studies have been conducted to examine mechanisms associated with DE effects on the immune system and host defense. In one study, Harrod et al. observed susceptibility to respiratory syncytial virus (RSV), a common respiratory pathogen in children. RSV gene expression in the lungs was increased following prior DE exposure as well as inflammatory and epithelial cell modulations consistent with reduced resistance to RSV, 
specifically a shift from normal cuboidal clara cell appearance to the presence of thickened columnar appearing mucus cells. These findings suggest a thickening of the airway. Additionally, reductions in clara cell-secreted, immunoregulatory proteins including clara cell secretory protein (CCSP) and surfactant protein A (SP-A) were shown to be modulated following co-exposure to DE and RSV. These findings indicated increased susceptibility to RSV following DE in a typicallyresistant mouse model [312]. Interestingly, in another study by Harrod et al. it is determined that $\mathrm{DE}$ inhalation resulted in reduced clearance of a bacterial infection, Pseudomonas aeruginosa (PA), in a concentration-dependent manner. Reductions in clearance were again determined to be related to inflammation and alterations in airway epithelial cell morphology, ciliated cells in the airway were decreased. Further, CCSP was reduced in clara cells of the distal airway following infection. Therefore, since the protein thyroid transcription factor (TTF)-1 has been shown to be an important transcription factor for lung host defense proteins including SPs and CCSP, the effect on TTF-1 was investigated. TTF-1 expression during infection was reduced following DE exposure. Altogether, these results point to DE exposure resulting in reduced respiratory resistance to PA infection [313]. Ciencewicki et al. examined the effect of DE on subsequent influenza infections, and showed that DE increased susceptibility to infection as determined by increased presence of hemagglutinin mRNA, and increased staining of viral proteins in the lung. Increased expression of IL-6 and decreased SPs occurred consistent with aforementioned studies. The findings indicate that DE increases susceptibility to viral infection by modulating host defense proteins [314]. Gowdy et al. demonstrated that exposure to occupational levels of DE caused increased lung injury, inflammation, and decreases in innate immune molecules including CCSP, and SPs consistent with Harrod et al. The authors determine that these finding point to possible decreases in respiratory resistance to infections [315].

Other investigators have expanded their studies to examine both branches of the immune system, examining both innate and adaptive alterations. Yin et al. studied the effect of DPM on the functions of AMs and lymphocytes from the lymph nodes of rats. In their first study, the authors exposed rats to DPM acutely and examined the clearance of bacteria from the lung following Listeria monocytogenes (LM) infection. It was determined that DPM inhibited phagocytosis of bacteria and reduced expression of cytokines from AMs. The authors also conclude that cellmediated immunity may be inhibited due to suppressed AMs effector functions [316]. In a followup study by Yin et al., the authors studied whether DPM had effects on T-cell development based on findings that AM function was altered by DPM in the presence of infection. It was found that DPM exposure resulted in decreased expression of IFN- $\gamma$, and that co-exposure to DPM and bacteria resulted in increased bacterial counts in the lung on day 3 post-infection. However, 
lymphocytes from these animals increased in number and were able to clear infection, despite alterations in innate immunity in an acute exposure to DPM [317]. Furthermore, Yin et al examined whether DPM exposure exerts a sustained effect on lymphocytes resulting in susceptibility to LM infection. Lymphocytes from infected animals previously exposed to DPM displayed decreased production of cytokines compared with animals exposed to bacteria only suggesting that DPM altered cytokine expression by lymphocytes. Further, it was found that DPM modified expression of cytokines by AMs that initiate lymphocyte development, including IL-1 $\beta$ and TNF- $\alpha$, as well as cytokines that suppress macrophage function including IL-10. Overall, it was concluded that DPM altered susceptibility to lung infection by inhibiting AM function and suppressing development of Th1 immune-mediated responses in the lung [318]. Similarly, Hiramatsu et al. demonstrated that long-term exposure to DE before infection with Mycobacterium tuberculosis resulted in increased lung burden due to decreases in IL-1 $\beta, I L-12$, IFN- $\gamma$ expression, and iNOS mRNA [319]. An investigation by Saito et al. showed that DE exposure resulted in modulation of cytokine mRNA expression and their corresponding secretion. It was found that levels of important inflammatory cytokines such as TNF- $\alpha$, IL- 6 , and IL-1 $\beta$ were down-regulated by exposure to DE; whereas, antiinflammatory cytokines like IL-10 were increased by DE exposure. Further IL-4 was increased by exposure to $D E$. These results indicate that $D E$ may alter immunological defenses while promoting allergic responses contributing to asthma [320]. Hahon et al. examined the role of DE exposure on susceptibility to infection with influenza. Higher levels of viral growth compared to control occurred animals exposed to DE. Additionally, depressed levels of interferon were uncovered in DE exposed groups. Further, antibodies to hemagglutinin were reduced in DE exposed animals compared with control. The authors concluded that results demonstrate reduced host defense to influenza following exposure to DE [321]. Overall, DE/DPM was shown to decrease host defenses. Both innate and adaptive immunity were hindered as decreases in phagocytosis, the expression of inflammatory cytokines by both AMs and lymphocytes, and decreases in antiviral antibodies were observed following DE/DPM exposure, although acute bacterial infections are cleared effectively despite these findings.

Studies have not only examined infection concurrently or subsequent to particle exposure but also while infection was ongoing in an effort to determine if DE exposure may result in exacerbated or prolonged infections. Gowdy et al. conducted experiments aimed to determine if DE would result in increased severity of and ongoing influenza infection. The authors state that DE exposure during viral infection resulted in increased viral titer, but that this effect was not due to reduced IFN levels, and that IL-4 expression was increased at early time points after exposure. Blockade of oxidative stress with an antioxidant did not reduce enhanced viral titers but blocked 
alterations in cytokine profiles and inflammation. It was concluded that exposure to DE during an active influenza infection polarizes the immune system to a Th2 response and that some of these effects may be reversed in the absence of oxidants [322]. A study by Larcombe et al. aimed at establishing whether DPM exposure could exacerbate existing infection reported that exposure to DPM following influenza infection resulted in increased viral titers and inflammation but not impairment of lung function. They concluded that although there was a presence of increased viral titers, one dose of DPM was not able to exacerbate respiratory disease [323].

Lastly, researchers have aimed to determine which components of DE may be responsible for the observed susceptibility to infection and alteration of immune functions described above. Yang et al investigated the potential of DPM to impair host defense to LM compared to CB, the core component of DE. Exposure to DPM decreased clearance of bacteria from the lungs and oxidants produced by phagocytes. These effects were not observed following exposure to CB and LM. Therefore, results suggest that suppression of host defenses by DPM are at least in part a result of components adsorbed to the core of DPM [324]. Castranova et al. compared different components of $\mathrm{DE}$ and $\mathrm{CB}$ in an effort to determine which properties of $\mathrm{DE}$ related to immunological alterations. Following their investigation, authors reported that exposure to DPM decreased bacterial clearance from the lung and that this was not observed with CB exposure. Further DPM reduced production of interferon and suppressed lung phagocyte production of oxidants, which was not observed with the control particle. The authors concluded that effects of DPM on host defense are likely related to organic components adsorbed to the carbon core of DPM [325]. Subsequently, Yin et al. explored whether DPM, specifically the organic extract or washed core particle resulted in ROS contributing to effects observed on AMs leading to increased intracellular infection. The authors reported that the organic extract of DPM but not the washed DPM particle elicited heme oxygenase (HO-1) expression and production of IL-10 and decreased expression of TNF- $\alpha$ and IL-12. Moreover, these effects were reversed by antioxidants [326].

\section{In Vitro Models of Diesel Exhaust Toxicity}

\section{Pulmonary Effects}

It is understood that DE causes an array of pathologies including; pulmonary-related disease, exacerbation of allergy, reduced immunity toward infectious insult, vascular-related diseases, and finally, under chronic exposure conditions, cancer [327]. What is less clearly understood, is which constituents of DE are responsible for provoking each specific response 
and the mechanisms of action of DE; however, oxidative stress, as a result of ROS production, has been demonstrated to be one of the integral mechanisms involved in these pathological signaling events [328]. In vitro models of toxicity have been employed to further delineated this pathway. Furthermore, in order to maintain focus on pulmonary disease, only those studies that pertain to respiratory pathology are summarized below.

Both carbonaceous and organic components of DPM have been implicated in the induction of oxidative stress. Dong demonstrated in the author's thesis work that DPM, washed DPM, and CB induced AM production of ROS; whereas, the organic extract from washed DPM failed to initiate a respiratory burst in AMs. Data from this study suggests that the carbonaceous component of DPM is at least partly responsible for activating AMs to initiate oxidative stress mechanisms leading to inflammation [327, 329]. Additionally, studies by Shukla et al. examined increased intracellular oxidative stress following DPM exposure in murine C10 alveolar cells. When comparing $\mathrm{PM}_{2.5}$ to both $\mathrm{CB}$ and glass beads, it was shown that both $\mathrm{PM}_{2.5}$ and $\mathrm{CB}$ stimulated intracellular oxidant production, while glass beads did not. These results also suggest that $\mathrm{PM}_{2.5}$ carbonaceous core components are capable of stimulating cells to produce ROS [330]. However, there is overwhelming evidence that the organic fraction of adsorbed chemicals, including PAHs on the surface of the carbon core, contributes to oxidant production, particularly through the intracellular pathway. Sagai et al. examined whether DPM could induce ROS production in an acellular system. The investigators were able to show that DPM released SO and hydroxyl radicals until washed with organic solvents. Also, this process could be inhibited by the addition of SOD or dimethylsulfoxide (DMSO). These results suggest that DPM toxicity is at least in part due to the presence of radicals and ROS, and that this process is driven by organic constituents adsorbed to particles [271]. Greenwell et al. compared the oxidative potential of coarse and fine fractions of DPM to CB using the Plasmid Assay. The goal was to examine the protective effects of surfactant and antioxidants against injurious inhalants. The authors established that the coarse fraction of DPM displayed a higher oxidative potential in this study and that soluble constituents were most directly responsible for toxicity. Moreover, it was found that antioxidants from lung lavage with low molecular weights offered some protection against DNA damage caused by oxidation, even when mimicked in a synthetic surrogate lung fluid [331]. Xiao et al. examined the effects of organic extracts of DPM on the induction of oxidative stress in mouse macrophages, examining newly formed proteins associated with stress pathways. The authors demonstrate a dose-dependent relationship with DPM-extract and induction of oxidative response as decrements of antioxidant ratios occured, suggesting that organic components of DPM are responsible for the upregulation of oxidative 
responses associated with exposure to pollutants [332]. Similar studies, by Jung et al., went on to highlight the importance of the unfolded protein response (UPR) in bronchial cells exposed to organic extracts from DPM using proteomics approaches. The results of this study suggested that oxidant damage caused by organic constituents of DE result in mis/unfolded proteins leading to inflammatory damage [333]. Contrasting results obtained between studies may be attributed to wide variations in dose, type of diesel fuel, or composition of extracts making it difficult to clearly attribute effects to any one component of DE. Taken together, these studies demonstrate that both the carbon core of DPM as well as the absorbed constituents are capable of initiating oxidative stress.

Reactive oxidants have further been associated with modulating the process of programmed cell death or apoptosis [332]. The pathways that drive this process following exposure have been the focus of several in vitro investigations. Hiura et al. demonstrated that DPM that had organic constituents extracted no longer caused macrophages to produce ROS that were shown in this study to be important for initiating the process of apoptosis in these cells following exposure [334]. Further, a subsequent study showed that ROS production by AMs induced by DPM extracts resulted in mitochondrial toxicity and the initiation of apoptosis mediated by mitochondrial damage [335]. The transcription factor NF-kB pathway is also known to be involved in regulation of apoptosis following response to ROS, as previously discussed in the context of silica exposure [79]. Therefore, Shukla et al. aimed to understand whether pollutants such as $\mathrm{PM}_{2.5}$ could initiate NF-KB translocation. The authors demonstrated that $\mathrm{PM}_{2.5}$ does cause translocation of NF-KB subunits into the nucleus and subsequent DNA binding in murine type-II alveolar epithelial cells. Further, they showed that this was dependent upon oxidative stress and resulted in the expression of increased inflammatory cytokines [330]. Bonvallot et al. showed the importance of NF- kB transcription of cytokines following exposure to DPM in human bronchial epithelial 16HBE cells. These authors added that activation of this pathway was dependent on oxidants produced by organic components of DPM, such as PAHs. Further, the mitogen-activated protein kinase/extracellular-signal related kinase (MAP/ERK or MEK) pathway was implicated in this process as DPM was shown to cause phosphorylation of ERK. However, inhibition of MEK did not always result in decreased NF-KB transcription in response to oxidant stress. Therefore, these pathways may not be directly linked to each other following DPM-induced oxidant stress [336]. Activation of NF-kB by DPM was increased by the combined exposure with $\mathrm{O}_{3}$, further demonstrating a role for ambient interactions in the environment worsening effects of air pollutants. The investigators indicated that increased NFKB activity in A549 (human airway epithelial-type II like) cells exposed to DPM and $\mathrm{O}_{3}$ resulted 
in increased expression of downstream cytokines including IL-8. IL-8 is a robust chemotactic factor for neutrophils, and is integral to the signaling mechanism for increased inflammation [337]. Effects of $\mathrm{O}_{3}$ in combination with DPM altered DPM pathology, as has been suggested in several studies [228, 239, 241, 276, 300]. Kaimul Ahsan et al. established that thioredoxin (TRX), a redox regulation protein and antioxidant was important for mitigating the effects of DPM-induced apoptosis. Results from this study suggest that DPM reduces phosphorylation of an anti-apoptotic protein, Akt1. TRX prevents this effect on Akt1 allowing for A549 lung cells to resist damage and resultant cellular death [338]. Conversely, Kafoury and Madden reported that DPM failed to initiate apoptosis despite activated NF-KB and triggered release of TNF- $\alpha$. Further, when activation of NF-KB was blocked, apoptosis was initiated by DPM. Conversely to others, the investigators conclude that NF-KB activation in response to DPM may actually have a protective role against apoptosis [339]. Differences may be dose-dependent, and are also likely specific to the type of DPM used in the studies and the relative amount of adsorbed organic constituents that are present. From the studies above, it may be concluded that the organic fraction may be strongly influencing the apoptotic pathway.

Trace metals associated with DPM have also been implicated in the production of ROS. Park et al. measured ROS production comparing two types of DPM to determine importance of transition metals in oxidative stress. The authors determined that iron was more important than other transition metals in the production of ROS in response to hydrogen peroxide [340]. Distefano et al. examined the role of transition metals in generating ROS in response to DPM exposure. They found that transition metals did play a role in redox activity of DPM using a newly described assay, where ascorbic acid is used to reduce metal ions and oxygen in order to generate $\mathrm{SO}$, which is then reduced to $\mathrm{OH}$ radicals in the presence of transition metals [341]. Ghio et al. further showed that ROS production by BEAS-2B cells in response to the soluble fraction of DPM does not account for total ROS production indicating that the insoluble fraction of particulates caused the generation of quantifiable ROS as well [342].

The study by Ghio et al. also highlights a major discrepancy between studies of this nature as described above. Studies examining the oxidative nature of components of DE resulted in findings that were inconsistent between similarly designed studies. This is due to several factors, the first of which being the complex nature of DE itself and the variable qualities of DPM from different sources and collection methods. Further, these samples were produced at different times of the year under variable conditions, resulting in environmental variability in constituents. Methodologies used to wash particles or extract particular fractions were not 
constant nor were methods used to quantify the generated oxidants. Culture conditions, cell types, and doses examined were also variable. Finally, the studies examined different species of ROS, i.e. SO, hydroxyl, peroxynitrite. A review by Ghio et al. summarizes these discrepancies [343]. All of these factors should be taken into consideration when drawing conclusions from this body of work and when making further determinations relating to DPM-generated oxidants and their role in the initiation of inflammation and pulmonary toxicity.

In vitro models have been employed in an effort to understand how exposure to DE alters respiratory responses observed in obstructive pulmonary disorders such as asthma and bronchitis, particularly in the context of pre-existing disease. In an ex vivo human study, Bayram et al. studied the differences in airway cell responses in cells recovered from asthmatics compared to non-atopic individuals, followed by exposure of the cells to DPM. Constitutively, cells differed in their expression of inflammatory mediators between the experimental groups, with asthmatic cells secreting significantly more mediators at baseline, though ciliary beat frequencies were determined to be similar regardless of atopic status. Further, it was determined that in response to DPM, bronchial epithelial cells of atopic individuals were more sensitive than those of nonasthmatic individuals, with regard to cytokine secretion. Moreover, this response was dose-dependent. The authors conclude that the altered expression of inflammatory mediators in response to DPM in asthmatics may contribute to exacerbation of symptoms [344].

From a mechanistic perspective, Li et al. examined the importance of matrix metalloproteinase (MMP)-1 in the development of obstructive diseases following exposure to DPM. MMP-1, like other MMPs, is a modulator of inflammation, tissue remodeling, wound healing, and pathogenesis in some pulmonary diseases[345]. The authors established that MMP-1 transcription was upregulated in primary human bronchial cells exposed to DPM, and that this was dependent upon the MEK signaling pathway. Further, a polymorphism identified in the MMP-1 promoter may increase susceptibility of many individuals exposed to DPM to developing respiratory diseases associated with increased MMP-1 secretion [346]. Another study by the group examined the involvement of calcium signaling in airway epithelial cells in response to DPM exposure in relation to COPD pathogenesis. This set of experiments further elucidated the link between DPM exposure and MMP-1 secretion resulting in obstructive pulmonary disease implicating the signaling pathway upstream from the MEK pathway involved in the influx of calcium following direct interaction between DPM and the receptor it initially activates upon contact with cilia on epithelial cells, proteinase-activated receptor (PAR)-2. Upon 
activating PAR-2, components of DPM upregulated PI3K signaling, which in turn upregulated influx of extracellular calcium by way of the transient receptor potential vanilloid (TRPV)-4. This was described as being dependent upon the organic fraction of DPM. Further, a polymorphism in TRPV4 already identified with enhanced susceptibility for COPD [347], increased the upregulation of MMP-1 activation described in the former study further [348].

Additionally studies have also focused on the effects of DPM on the COPD characteristics of airway mucus hypersecretion and stimulation of C-fibers in airway reactivity. In an effort to better understand increased secretion of mucus in the airway following exposure to pollutants, Park et al. designed studies for evaluating expression of the gene for mucin 4 (MUC4) in nasal epithelium and human lung mucoepidermoid cells ( $\mathrm{NCl}-\mathrm{H} 292)$. The authors determined that exposure to DPM increased the mRNA expression and protein secretion of MUC4. Further, these results were reversed when proteins in the MAPK pathway or the cAMP response element-binding protein pathway (CREB) were blocked. This occurred in both cell types. These findings reveal a mechanistic understanding for hypersecretion of mucus in airway epithelial cells following exposure to DPM [349]. More recently, the respiratory reflexes and more specifically the innervation of the airway have been examined in response to DPM. Robinson et al. exposed airway C-fiber afferents to DPM and observed direct activation. The results of this study suggest that activation of the ahR causes production of ROS by mitochondria and the downstream activation of the transient receptor potential ankyrin-1 (TRPA1) followed by depolarization of the vagal nerve $c$-fibers causing an action potential that is experienced in the form of a cough or bronchospasm. Further, washing the particles of the organic components abrogated this activation of the vagus nerve, suggesting that the organic PAHs are primarily responsible for observed effects [350].

Due to the rise in asthma and allergic prevalence over the past few decades worldwide, efforts have been made to advance the understanding of DE exposure in the development of allergies and allergic asthma as discussed above for the reviewed in vivo studies. In vitro studies have also been performed to further investigate mechanisms of DE exposure in cells involved in allergic response. Terada et al. established that DPM organic extracts were able to enhance the adherence of eosinophils to nasal epithelium. Further, their results demonstrated that DPM extracts induced eosinophilic degranulation though not altering eosinophilic viability. These findings lead the authors to conclude that DPM, more specifically PAH components of DPM, play a role in nasal hypersensitivity and the development of allergic response [351]. The same group further demonstrated the role of DPM in allergic responses by elucidating the 
effects of DPM on the expression of the histamine $\mathrm{H} 1$ receptor $(\mathrm{H} 1 \mathrm{R})$. Their experiments demonstrated that extracts of DPM increased H1R mRNA expression in nasal epithelium and mucosal endothelial cells. Further, the secretion of IL-8 and GM-CSF stimulated by histamine was higher in cells treated with DPM than controls. The results of these investigations suggest that organic components of DPM upregulate allergic inflammation by increasing the release of histamine [352].

Studies have also focused on the involvement of lymphocytes in the development of allergic disease and the skew toward a Th2-mediated response. Though these cells are important for upregulation and induction of this response, other immune cells have been shown to be important for polarizing lymphocytes toward allergy. Saneyoshi et al. conducted a study to determine the role of environmental pollutants in development of allergic disease by examining the effect of DPM on mast cells. Results from this study suggested that low levels of DPM were capable of enhancing the expression of IL-4, which is important for the differentiation of T cells during allergic response. However, interestingly, it was determined that this was dependent upon time of exposure relative to activation state of the mast cells. Further, it was shown that the enhanced expression of IL-4 may not be entirely dependent on oxidant production, suggesting other mechanisms for allergic induction following exposure to DPM [353]. Devouassoux et al. examined the effects of DPM organic constituents on basophils, since basophils are a major source of IL-4, and IL-4 is important for the polarization of Th2 mediated responses. Basophils exposed to extracts of DPM displayed enhanced expression of IL-4 and increased release of histamine compared with basophils exposed to allergen. However, no synergy was observed when cells were co-exposed to DPM extracts and allergen. Additionally, secretion of IL-4 by basophils exposed to DPM extract persisted for longer periods of time than those stimulated with allergen only. Furthermore, authors demonstrated that IL-4 expression was not altered by alternative cell types suggesting that basophils were the major contributors to the increase observed. Finally, the study showed that antioxidant pre-treatment of basophils reversed the expression of IL-4 observed in response to DPM extract treatment establishing that ROS are involved in the initiation of allergic responses of basophils to pollutants [354].

Nilsen et al. investigated the importance of Th1 polarizing cytokine, IL-12, in the involvement of allergic response. The authors determined that levels of IL-12 expressed by activated monocytes were inversely associated with concentration of DPM exposure. Suppression of IL-12 expression by monocytes could skew the Th1/Th2 balance in favor of a Th2 dominated response providing further mechanisms for DPM-induced atopy [355]. 
Mamessier et al. further explored the role of $\mathrm{T}$ cell activation involvement in allergic response to DPM. It was determined that $T$ cell activation and proliferation, specifically IL-4 producing $T$ cells, was increased in higher proportions in cells obtained from asthmatics compared with healthy controls. These results were exacerbated for cells obtained from severely uncontrolled asthmatics suggesting an increased susceptibility for respiratory symptoms following exposure to DPM in these individuals [356]. A study conducted by Takenaka et al. determined that while the organic components of DPM were able to enhance IgE production by B lymphocytes. PAHs from DPM were not inherently able to induce lgE production or class switching of immunoglobulin. Therefore, it was concluded that DPM exposure plays a role in upregulating ongoing allergic responses [357]. Plé et al. more recently determined that the Th17/Th22 axis may play a role in allergic response following exposure to air pollutants. The authors exposed monocytes from either allergic asthmatics or non-asthmatics to DPM PAHs in order to determine whether the organic constituents of DPM play a role in the up-regulation of allergic response by way IL-17 and IL-22 expression. IL-17 and IL-22 were both expressed more highly in the monocytes of asthmatics at baseline. Exposure to DPM PAHs increased expression of IL22 while decreasing IL-17 expression regardless of predisposition to disease. The increase of IL-22 was found to be partly dependent on up-regulation of the ahR transcription factor [358]. These studies indicate a role for DPM, and specifically the organic components of DPM for upregulating the expression of cytokines important for Th2 response; further, these results were worse in individuals predisposed to allergy/asthma suggesting that DPM may exacerbate ongoing allergic responses.

Additionally, several ex vivo studies in addition to those of Mamessier et al. and Bayram et al. described above, have also aimed to elucidate mechanisms leading to enhanced allergic response caused by DPM. Similar to work previously described by Bayram et al., a study by Devalia et al. examined the mechanisms associated with enhanced sensitivity of airways in asthmatics. By exposing bronchial cells of asthmatics and non-atopics to DPM and comparing the secretion of inflammatory mediators to constitutive secretion of the same mediators, it was determined that bronchial cells of asthmatics were more sensitive to DPM at lower doses and overall had higher constitutive secretion of inflammatory mediators than those of non-atopic individuals [359]. Fahy et al. aimed to understand whether organic components of DPM in concert with HDM allergen could result in hyperresponsiveness in atopics. DPM PAHs and HDM allergen both elicited increased allergic mediators in monocytes from allergic patients. Moreover, when co-exposed to DPM PAHs and HDM allergen, synergistic responses were observed. Further, MEK pathway activation was implicated in the role of increased expression 
of mediators detected in response to co-exposure. The authors conclude that DPM PAHs and allergens act jointly on the MEK signaling pathway to increase expression of inflammatory and chemotactic proteins important in the development of hyperresponsiveness [360]. To further investigate this, Fahy et al. exposed monocytes from HDM allergic patients to combined DPM and allergen stimulants and observed expression of monocyte-derived chemokine (MDC), a recruiter of Th2 cells, and IFN-y-induced protein 10 (IP-10), a Th1 cell recruiter, to determine if DPM might skew recruitment of T lymphocytes to allergic response. It was found that DPM exposure alone resulted in down-regulation of IP-10 expression constitutively while exposure to DPM in combination with HDM allergen further increased expression of MDC observed with exposure to HDM alone. Additionally, blockade of the CD28 and CD86 pathways abrogated increased MDC expression suggesting that this adjuvant effect is in part caused by Th2 polarizing chemokine milieu dependent upon CD28 signaling, though this effect was also reduced by blockade of CD80, it was to a lesser extent. Further, these results were found to be independent of IL-4, IL-13 and IFN-y expression [361]. Chang et al. sought to examine whether allergic responses were not only increased by contributions from DPM or whether DPM may enhance predisposition toward allergic response even in previously non-atopic individuals. The authors designed experiments examining the stimulation of either Th1 or Th2 chemokines in response to DPM in peripheral blood monocytes of non-atopic individuals. Results from this study suggest that DPM exposure induces expression of chemokines that are involved in the recruitment of Th2 lymphocytes and down-regulation of chemokines that favor Th1 recruitment, suggesting that even in non-atopic individuals DE exposure may result in the induction of allergic responses [362]. These studies demonstrate that DPM, by up-regulating inflammatory signaling may result in increased hypersensitivity and allergic response; further, this may be related to organic components of DPM skewing response of the immune system from Th1 mediated to Th2 mediated especially in the presence of allergen.

Don Porto Carero et al. conducted studies to examine the role of pollutants in altering expression of antigen presentation receptors as a mechanism in DPM-induced allergic response. Human THP-1 monocytes were stimulated with environmental pollutants including DPM simultaneously with IFN- $y$ in order to determine if pollutant-altered antigen presentation might be responsible for adjuvant effects during allergic response. Although pollutants did not stimulate expression of antigen presentation receptors on their own, they potentiated effects of IFN- $y$ induced expression of these receptors. These investigators state the results suggested a mechanism for adjuvant effect observed for environmental pollutants during allergic disease. Additionally, the authors speculated that particle size and surface area relative to dose is critical 
for these effects to occur based on varied responses between DPM and urban particulate matter [363]. Similar to the previous study, Takizawa et al. examined the role of antigen presentation following exposure to DPM in the development of allergic response. The authors specifically aimed to determine whether nasal epithelium cells (NEC) were able to act as APC during allergic response and if DPM altered this phenotype. Experiments demonstrated that NEC expressed APC-related receptors and that compared with pre-seasonal allergy, these receptors were upregulated in the NEC of patients during allergy season. Further, exposure of these NEC to DPM resulted in further increases in the expression of these receptors. These results were dependent upon the CD86 pathway, and not the CD80 pathways, similar to the study by Fahy et al. Co-exposure to allergen with DPM further increased the expression of APC molecules in NEC, and initiated the proliferation of lymphocytes in response to NECs. Blockade of APC receptors and CD86 pathway abrogated proliferation of T-cells. Overall, the results demonstrate the role of NEC as APCs in allergic response to antigen and DPM [364].

Dendritic cells are also important for the differentiation of T cells. A study by Chan et al. examined the effect of DPM on DCs and how this may influence the adjuvant effects of DE exposure in the development of allergic asthma. This study demonstrated that DPM elicited oxidant-related stress on DCs. Oxidants were shown to interfere with the TLR-mediated expression of receptors and the production of cytokines including IL-12. These changes resulted in altered interaction between DCs and lymphocytes, and therefore aberrant T cell differentiation shifted toward a Th2 response. Further, the authors were able to determine that activation of nuclear factor-erythroid 2 (NF-E2)-related factor 2 was responsible for the altered DC function. NF-E2-related factor 2 expression suppresses IL-12. Blockade of this axis resulted in restored DC function in the presence of DPM exposure. Therefore, altered DC function is essential in the adjuvant effects of DPM [365]. Another study by Braun et al. examining the role of DCs in allergic response investigated whether exposure of DCs to DPM in the presence or absence of LPS stimulations would affect cell function or viability. Authors examined multiple types of DPM at varying doses and concluded that only DPM with high levels of adsorbed pollutants (as from traditional DE), were capable of altering DC phenotypes. DPM with high pollutants resulted in altered DC expression of cytokines, as well as, inability to suppress immunoglobulin class switching in B cells. Further they alter DC maturation. The authors conclude that aberrant DC maturation and function contributes to the Th2 favoring environment observed following exposure to highly polluted DPM, but that organic components may not be responsible for these effects [366]. Taken together, these studies suggest that DE exposure up-regulated allergic response in atopic individuals but also may play a role in induction of allergic response in 
previously non-atopic individuals. Furthermore, though many mechanisms were investigated experimentally, the conclusions were in general agreement that allergic responses are related to DPM constituents that alter antigen presenting cell responses and shift the inflammatory response to one that is Th2 driven. Additionally, the components of DPM most likely responsible are those adsorbed to the core and not carbonaceous particles themselves.

Though evidence of DE elicited carcinogenesis is conflicting in in vivo studies, in vitro studies allow for investigations in more varied cellular systems and cultures including human cell lines. These studies provide more mechanistic implications for the pathogenesis of DE exposure and for the development of cancer, beginning with mutagenicity testing upon the development of the Ames S. typhimurium assay. [367]. Therefore, after early evidence that DPM causes mutagenicity [368-371], further analysis has been conducted to determine carcinogenicity including more modern assays including but not limited to micronuclei assay, cell cycle assay, and DNA damage analysis.

Bieler et al. examined a known mutagen from diesel exhaust, 3-nitrobenzathrone (NBA) and determined that it was able to covalently bind DNA and create DNA adducts in an acellular system. Ultimately, these adducts may result in carcinogenesis, supporting that organic fractions of DPM may be tumorigenic [372]. Further, Shi et al. examined DPM mutagenicity using the Ames assay following alterations to engine operating conditions and using filters for particulate. Mutagenicity was found to be strongly correlated with engine operating condition and although filtration reduced the concentration-normalized mutagenicity of DPM, it is likely that mutagenicity is dependent upon the organic fraction of DPM that was allowed to pass through the filters [373].

Arimoto et al. explained how production of oxidative stress by DPM components could lead to carcinogenesis. DPM generated oxidative radicals in biological systems due to enzyme activation by $\mathrm{P} 450$ reductase. These radicals hydroxylated guanine residues causing 8-hydroxy2'-deoxyguanosine (8-OHdG) lesions in DNA, lesions that are thought to lead to tumorigenesis [374]. Don Porto Carero et al. also demonstrated that DPM as well as CB caused DNA damage following exposure to non-cytotoxic doses in two human cell lines. Therefore, it is plausible that the organic fraction of DPM is not the only genotoxic fraction of DE, though the organic extract was shown to be genotoxic as well as whole particulate. These results suggest that carbonaceous constituents of DPM may be partly responsible for the carcinogenesis of these substances observed in vivo and in humans [375]. 
Jardim et al. exposed human bronchial cells to DPM and examined expression of micro RNAs (miRNAs) to determine if miRNAs might have a role in tumorigenesis of DPM. miRNAs altered to the greatest degree were related to inflammatory pathways and tumorigenesis. Further, DPM disruption of miRNA expression patterns was significant, leading the authors to conclude that cellular homeostasis is generally affected by alterations in gene expression regulated by miRNAs [376]. Zhou et al. also reported on the importance of miRNA in the development of malignancy following exposure to DPM. The authors describe a novel pathway for carcinogenesis caused by DPM through dysregulation of miRNA, miR-21. It was established that upregulation of miR-21 by DPM leads to downregulation of phosphatase and tensin homolog (PTEN) that suppresses PI3K/AKT. The PI3K/AKT pathway has anti-apoptotic activity; therefore, upregulation of PI3K/AKT activity by miR-21 acting on target protein PTEN results in an anti-apoptotic mechanism for cell persistence by evading programmed death [377]. A specific amino acid transporter (LAT1/CD98hc) has been identified as upregulated in lung cancers and is associated with poor prognosis [378, 379]. Le Vee et al. demonstrated that exposure of bronchial epithelial cells to DPM extracts resulted in dose-dependent expression of LAT1/CD98hc. This was found also to be dependent upon activation through the ahR pathway, as inhibition of ahR prevented LAT1/CD98hc induction caused by DPM extracts. These results suggest that DPM extracts initiate tumor promoting pathways through ahR pathways [380]. A study by Yadav et al. demonstrated how a mechanism that protects cells from toxicity caused by DPM may ultimately lead to tumorigenesis. Ubiquilin (UBQLN) proteins aid in the degradation of ubiquitinated proteins and their gene expression has been found to be down-regulated in lung cancers. In fact, loss of UBQLN1 may result in EMT [381]. The current study established that exposure to DPM resulted in the upregulation of UBQLN proteins overall in two cancer cell lines and that this was dependent on the suppression of miR-155. Conversely, over-expression of miR-155 resulted in EMT in lung cancer cells by downregulation of UBQLN1/2. The authors speculate that initial increases in UBQLN1 and reductions in miR-155 are part of an acute stress response to DPM exposure leading to an eventual feedback mechanism causing the selective deletion of UBQLN1 by increased miR-155 expression during chronic exposure [382].

Bao et al. determined that some mutagenicity by DPM is reliant on cell particle interaction requiring the internalization of particles by the cells. The investigators exposed human-hamster hybrid cells to DPM and examined increases in mutagenicity that were dosedependent. Further, when phagocytosis was blocked, mutations induced in the nuclei were reduced. These results suggest that ingestion of particles and radicals produced in response to this process may be responsible for initiation of carcinogenic processes [383]. An investigation 
led by Landvik found that nitrated PAHs ( $\mathrm{n}-\mathrm{PAHs}$ ) commonly found in DPM have varying effects on mutagenicity and induction of apoptosis. While all n-PAHs induced some mutagenicity, most were pro-apoptotic due to increased phosphorylation of p53 or decreases in anti-apoptotic bcl$\mathrm{XL}$. However, the authors concluded that the most mutagenic n-PAH examined, 1,8dinitropyrene (1,8-DNP), resulted in the least apoptosis induction. 1,8-DNP inactivated p53 by a pathway that was determined to be non-mutagenic. This study highlights the importance of individual components of DPM in health outcomes as this n-PAH is only present in some DPM based on the conditions in which the engine was run. Further, these results suggest that increased cell survival occurs with highly damaged DNA providing a mechanism for carcinogenesis following exposure to DPM-related substances [384]. Matsunaga et al. identified an aldo-keto reductase (AKR) involved in the progression of tumor growth associated with exposure to a quinone component of DE. They found that exposure of human lung cells to high concentrations of 9,10-phenanthrenequinone $(9,10-\mathrm{PQ})$ resulted in apoptosis; whereas, exposure to lower concentrations led to proliferation and invasion. This was determined to be related to upregulation of AKR1B10; inhibition of AKR1B10 abrogated tumorigenic processes in 9,10-PQ treated cells. Further the carcinogenic activity promoted by AKR1B10 was determined to be dependent on the production of ROS due to disruptions in the redox balance by 9,10-PQ. Altogether, these results from this study demonstrated a newly defined pathway for cancer promotion by components of DE [385].

Overall, mechanisms investigated for pulmonary carcinogenesis suggest that imbalances of redox systems result in DNA damage and initiation of pathway signaling associated with anti-apoptotic mechanisms. Together these effects result in dysregulation of cellular homeostasis and the persistence of aberrant cells. Further studies are needed to elucidate the multi-phase toxicological responses to air pollutants.

\section{Immunological Effects and Susceptibility to Infection}

Due to epidemiological observations that point to air pollution and DE exposure resulting in increased hospitalizations for pulmonary infections, many investigations have aimed to determine more causal mechanistic relationships between $D E$ exposure and degradation of host defense. Thomas et al. examined peripheral monocytes and AMs exposed to either 2 or 200 $\mu \mathrm{g} / \mathrm{ml}$ of DPM in suspended in culture medium to determine whether DPM might alter phagocytosis mechanisms and/or secretion of TNF- $\alpha$ as parameters of effector functions compared with control cells stimulated with $5 \mathrm{ng} / \mathrm{ml}$ LPS. Though no change in cell viability or attachment was observed, reductions in phagocytosis by cells exposed to DPM occurred in a 
dose-dependent manner [386]. Castranova et al. compared the effects of either DPM or organic extracts from DPM on the function of AMs in the context of their production of cytokines. Results showed that macrophages exposed to DPM were less able to respond to LPS, namely in the production of TNF- $\alpha$. Further, methanol extracts of DPM were able to suppress cytokine expression in response to LPS, whereas washed DPM was not, suggesting that these effects were driven by the organic components adsorbed to DPM [325]. Saito et al. determined if DE exposure altered the ability of AMs to produce cytokines in the absence and presence of intracellular infection. The authors determined that the production of cytokines by AMs in response to DE was altered. IL-12 and TNF- $\alpha$ expression was decreased, while the secretion of IL-1 $\beta$ was increased. They also determined that expression of apoptotic factors was increased in AMs following DE exposure. The effects observed in the absence of infection were more pronounced following exposure to bacterial infection. These results suggest that DE exposure may reduce the AMs' effectiveness in controlling infection, thereby, increasing susceptibility to infection [387]. Lundborg et al. established that AMs exposed to aggregates of ultrafine carbon particles and DPM diminish phagocytosis mechanisms. Further, phagocytosis was altered for receptors typically stimulated by four distinct ligands suggesting that phagocytosis was universally altered, and that this effect was not specific for one receptor-type relating to this mechanism. Since phagocytosis is crucial in the control and clearance of many pathogenic substances in the lung these findings provide important evidence for pulmonary susceptibility following exposure to particulate matter [388].

Yin et al exposed AMs to DPM, its organic extracts, washed DPM (wDPM), or CB particles. Their results demonstrated that DPM and organic extracts decreased phagocytosis and bactericidal responses to LM by AM. wDPM and CB were also able to decrease phagocytosis. However, while CBs did not decrease bacterial killing by AMs, wDPM did affect bactericidal activity, but also not as significantly as DPM or its extracts. DPM and its extracts also decreased the expression of inflammatory proteins by AMs. Other particles were not effective in altering the expression of inflammatory proteins. These collective results provide evidence that organic components of DPM are instrumental in diminishing important antimicrobial activities of AMs and thus enhance respiratory susceptibility to infection [389]. Sarkar et al. examined the effect of DPM on inflammatory signaling pathways during mycobacterial infection in human monocytes. The authors demonstrate that DPM suppressed the expression of inflammatory cytokines, IFN- $\gamma$, TNF- $\alpha$, IL-1 $\beta$ and IL- 6 , in response to mycobacterium in a dose-dependent manner. DPM treatment of cells altered the expression of many NF-KB, TLR, and IFN constituent mRNAs as well. These results jointly suggest that 
cellular response to mycobacterial infection following DPM exposure is altered in favor of decreased resistance [390]. Since NK cells are known to be located in nasal mucosa, Müller et al. investigated their role in defense against viral infection and whether DPM altered these activities. The authors conclude that DPM may reduce the ability of NK cells to kill host cells that are infected with virus since they lose important expression of cytotoxic factors granzyme $B$ and perforin when exposed to DPM, results that suggest diminished host defenses [391]. Altogether, these findings suggest that DPM reduce resistance to infection and that both the core and adsorbed components of DPM may play a role in these effects.

Effects of DE on susceptibility to infection have also been examined in epithelial cells. Jaspers et al. infected respiratory epithelial cells with influenza following exposure to DE and established that exposure to DE enhanced the susceptibility to influenza infection in all cell types tested. Moreover, this was determined to be due to enhanced attachment and entry into cells. The effects observed were also dependent upon oxidative stress generated by DE as antioxidant treatment reversed the infectious effects observed by DE exposure. Lastly, the suppression of host resistance to influenza infection was shown to be independent of effects on interferon [392]. Ito et al. also examined the effect of DPM exposure on rat lung alveolar type-II epithelial cells. The authors observed up-regulations in mRNA expression of sites identified with bacterial and viral invasion. They established that expression of these receptors were increased in a concentration-and oxidative stress-dependent manner. Overall, the results of this study demonstrate a mechanism by which pathogens may invade the site of the lung more efficiently leading to increased host susceptibility [393]. Similarly, Ciencewicki et al. revealed that DE exposure of lung epithelial cells resulted in enhanced TLR3 expression and function that may provide opportunity for the increased pathogen invasion and susceptibility observed by Jaspers et al. [394].

Taken in conjunction, the results of these studies consistently show that DPM, specifically the organic constituents associated with DPM, causes decreases in global phagocytosis, and reduce the ability of immune cells to secrete inflammatory mediators in response to pathogens. Further, evidence is provided that DPM upregulates certain receptors associated with bacterial and viral invasion of host cells. Therefore, in vitro systems provide mechanistic support for the susceptibility to infections observed in vivo as well as in human epidemiology associated with DE associated exposures. 


\section{Co-Exposure to Diesel and Silica}

The literature summarized here is an overview of the extensive body of investigations that examine exposure to silica and DE as individual exposures. Co-exposure studies investigating the health effects of $D E$ in conjunction with other exposures, or silica in combination with other exposures have been studied and primarily focus on ozone or asbestos [395-398]. However to date, no studies have specifically examined a co-exposure of crystalline silica and diesel. There are, however, a small battery of studies that examine DE in a coexposure with coal dust, which contains crystalline silica. In terms of epidemiological studies, a few investigations have examined the role of diesel exhaust and the incidence of lung cancer in coal miners; however, due to the lack of exposure related data, or the classification of exposure based on job category, it is difficult to determine a causal relationship. Furthermore, coincidence of confounding exposure including cigarette smoking needs to be considered [399, 400] . A chronic combined inhalation exposure evaluating $D E$ and coal dust (CD) in rats found exposure related lesions consistent with simple coal worker's pneumoconiosis, but this finding was not significantly different between diesel exhaust, high concentration coal dust, or the combination the two dusts. This finding was found to be time-dependent as longer exposures resulted in worsened severity of lesions [401]. However, this study combined doses of particles much higher than are permissible or consistent with underground survey measurements of particles.

Another set of studies examined DE in combined inhalation exposures with $C D$ in rats at exposure levels consistent with permissible exposure levels for particles $\left(2 \mathrm{mg} / \mathrm{m}^{3}\right)$ underground at that time. Many aspects of toxicity including AM function, airway function, cardiac and arterial pathologies, and liver and lung enzyme function were evaluated during a two-year chronic exposure to either DE $\left(2 \mathrm{mg} / \mathrm{m}^{3}\right)$, coal dust $\left(2 \mathrm{mg} / \mathrm{m}^{3}\right)$, or a combination of $D E\left(1 \mathrm{mg} / \mathrm{m}^{3}\right)$ and $C D$ $\left(1 \mathrm{mg} / \mathrm{m}^{3}\right)$. Castranova et al. determined that $\mathrm{DE}$, when in combination with coal dust was not cytotoxic to AMs at the relatively low doses administered over the course of 2 years. However, DE did appear to suppress the phagocytic activity of AMs while coal dust increased it. Combining the exposures resulted in no significant enhancement in overall macrophages recovered but may be due to the reduction in $C D$ administered in the combined exposure compared with the $C D$ exposure alone [402]. From this same study, Vallyathan et al. reported that there was no evidence that combined or separate exposure to $D E$ or $C D$ resulted in detrimental effects to the heart or pulmonary arteries [251]. Lewis et al. published findings from this same study reporting similarly to Castranova et al. that DE suppressed macrophage function while $\mathrm{CD}$ enhanced these functions. Additonally DE contributed to increased 
susceptibility to influenza infection and generally caused more health effects than did CD. While some alveolar epithelial hyperplasia and lipidosis was noted for individual exposures, these findings were not exacerbated by the combination of DE and CD. Furthermore, no evidence of obstructive or restrictive alterations were noted. Overall, this battery of studies suggested that combined exposure to DE and CD does not result in additive or enhanced risk beyond that of each component alone [403]. However, it should be noted that in this study, the co-exposure dose was normalized to total particle equivalent to that of DE or CD alone, resulting in lower concentrations of each in the co-exposure when compared to the individual dust exposures, which potentially attenuate particle-specific effects in the co-exposure group.

Finally, in a separate study, Hahon et al. demonstrated that mice exposed to DE (2 $\mathrm{mg} / \mathrm{m}^{3}$ ) alone caused increased severity of a subsequent influenza infection, but that exposure to combined DE $\left(1 \mathrm{mg} / \mathrm{m}^{3}\right)$ with CD $\left(1 \mathrm{mg} / \mathrm{m}^{3}\right)$ did not significantly alter this effect. Further, these responses were most significant following 3-6 months of previous DE exposure [321]. Overall, these findings suggest findings similar to those previously mentioned that demonstrate that DE may diminish immune responses. While these results do not significantly implicate co-exposure to $D E$ and $C D$ as causing altered pulmonary toxicity in response beyond that observed for each particle individually, this study examined relatively low doses that were given over a long period of time. Furthermore, while coal dust does contain quartz, amounts are drastically variable based upon type and location of extraction, and effects in the CD groups cannot be directly correlated to the silica component in the studies described above. Therefore, further studies examining acute responses, as well as studies that evaluate DE in combination with higher concentrations of silica are necessary in order to more completely evaluate the potential combined toxicity of co-exposure to these particles.

\section{Summary}

To this day exposures to silica in the workplace are documented to occur in a numerous occupations in addition to mining including masonry, painting, sand blasting, construction, demolition, and ceramics [404, 405]. Inhalation of respirable silica has been shown to cause lung inflammation and injury, COPD, bronchitis, emphysema, fibrosis, silicotic pneumoconiosis, cancer, heart disease, autoimmune disorders, and increased risk for infection in humans [18, $35,404,406-409]$, depending upon duration and level of exposure.

DPM exposure in occupational settings is as long-standing as the use of the combustion engine for industrial purposes, though exposure-associated health effects have only been 
recognized in the last few decades including airway inflammation, bronchitis, altered lung and vascular function, and the development of cancer [234, 410-415]. The complex nature of the chemical mixture that comprises DE inherently makes it difficult to study in controlled settings.

Although the health effects of both particles individually have been reviewed at length; there is very little information available on effects of co-exposure to silica and diesel. The current studies were designed to address the risk for enhanced pulmonary fibrosis and alterations in innate immunity related to susceptibility to infection following a co-exposure to crystalline silica and DPM administered at doses related to industrial settings with three major aims: (1a) characterize the pulmonary response to an acute co-exposure to DPM and SIL at varying doses; (1b) characterize the pulmonary response to a repeated co-exposure to DPM and SIL in order to determine if subacute exposure vs. acute exposure altered the effects observed for $1 \mathrm{a}$; (2) establish if susceptibility to an acute respiratory infection was altered following a co-exposure of DPM and SIL; and (3) determine whether clearance of particulate in a co-exposure to DPM and SIL was altered compared with the clearance rate of either particle individually to assess the effect of particle load in toxicity. 
Table 1: Retrospective Studies of Occupational Exposure to Silica Dust with Regard to Pulmonary Fibrosis

\begin{tabular}{|c|c|c|c|c|c|}
\hline $\begin{array}{l}\text { Study } \\
\text { Design }\end{array}$ & $\begin{array}{l}\text { Occupation } \\
\text { Examined }\end{array}$ & $\begin{array}{l}\text { Participant } \\
\text { Number (n) }\end{array}$ & $\begin{array}{l}\text { Exposure Level } \\
\text { (Average Time) }\end{array}$ & Findings & Citation \\
\hline $\begin{array}{l}\text { Case } \\
\text { Study }\end{array}$ & Bentonite Milling & 8 & $\begin{array}{l}2-10 \text { times the } \\
\text { TLV (10.5 years) }\end{array}$ & $\begin{array}{l}\text { Examination of } 8 \text { workers from a } \\
\text { bentonite milling mine revealed } \\
\text { incidence of findings consistent } \\
\text { with silicosis; one case was } \\
\text { confirmed by histological analysis; } \\
3 \text { surveyed workers had PMF, and } \\
4 \text { coincidence of tuberculosis } \\
\text { (TB). }\end{array}$ & {$[416]$} \\
\hline $\begin{array}{l}\text { Case } \\
\text { Study }\end{array}$ & Sandblasters & 4 & $\begin{array}{l}5 \text { times the TLV } \\
\text { (35 months) }\end{array}$ & $\begin{array}{l}\text { Acute silicosis, fibrotic lesions in } \\
\text { all, co-morbidity with cor } \\
\text { pulmonale and autoimmune } \\
\text { developments }\end{array}$ & {$[417]$} \\
\hline \multirow[t]{2}{*}{$\begin{array}{l}\text { Case } \\
\text { Study }\end{array}$} & \multirow[t]{2}{*}{ Stone Masonry } & \multirow[t]{2}{*}{2} & $\begin{array}{l}\text { Patient } 1: 0.1 \\
\mathrm{mg} / \mathrm{m}^{3} \text { ( } 30 \text { years) } \\
\text { followed by } 2 \\
\left.\mathrm{mg} / \mathrm{m}^{3} \text { ( } 5 \text { years }\right)\end{array}$ & \multirow[t]{2}{*}{$\begin{array}{l}\text { Low quartz exposure may } \\
\text { contribute to hilar node fibrosis, } \\
\text { which may lead to PMF following } \\
\text { subsequent high exposure to } \\
\text { silica }\end{array}$} & \multirow[t]{2}{*}[418]{} \\
\hline & & & $\begin{array}{l}\text { Patient } 2: 1.5 \\
\mathrm{mg} / \mathrm{m}^{3} \text { (6 years) }\end{array}$ & & \\
\hline $\begin{array}{l}\text { Case } \\
\text { Study }\end{array}$ & $\begin{array}{l}\text { Counter Top } \\
\text { Fabrication }\end{array}$ & 1 & n/a (10 years) & $\begin{array}{l}37 \text { year-old worker diagnosed with } \\
\text { silicosis advanced to PMF } \\
\text { following } 10 \text { year history of } \\
\text { polishing and laminating quartz } \\
\text { countertops. }\end{array}$ & [419] \\
\hline
\end{tabular}




\begin{tabular}{|c|c|c|c|c|}
\hline $\begin{array}{l}\text { Case } \\
\text { Study }\end{array}$ & $\begin{array}{l}\text { Surface Coal } \\
\text { Mining }\end{array}$ & 8 & n/a (32.9 years) & $\begin{array}{l}\text { High exposure to silica in surface } \\
\text { coal miners resulted in } \\
\text { pneumoconiosis with progression } \\
\text { to PMF }\end{array}$ \\
\hline $\begin{array}{l}\text { Case } \\
\text { Study }\end{array}$ & Stone Masonry & 6 & n/a (13 years) & $\begin{array}{l}\text { Though cases all had low } \\
\text { predicted probability of } \\
\text { pneumoconiosis based on health } \\
\text { survey, all had radiological } \\
\text { evidence of silicosis with half } \\
\text { presenting with asymptomatic } \\
\text { PMF upon screening exhibiting } \\
\text { the importance of early } \\
\text { radiological screening }\end{array}$ \\
\hline $\begin{array}{l}\text { Medical } \\
\text { Survey }\end{array}$ & $\begin{array}{c}\text { Silica Mining and } \\
\text { Milling }\end{array}$ & 86 & $\begin{array}{l}\text { Above TLV }(8 \\
\text { years for those } \\
\text { with silicosis) }\end{array}$ & $\begin{array}{l}39 \% \text { of workers with a mean of } \\
7.7 \text { years of work had simple } \\
\text { silicosis and } 16 \% \text { of workers with } \\
\text { a mean of } 7.1 \text { years of work had } \\
\text { PMF, determined to be due to } \\
\text { high exposure levels of high } \\
\text { percentage silica dust and lack of } \\
\text { effective controls }\end{array}$ \\
\hline $\begin{array}{l}\text { Medical } \\
\text { Survey }\end{array}$ & $\begin{array}{c}\text { Slate Pencil } \\
\text { Manufacturing }\end{array}$ & 593 & $\begin{array}{c}46.47 \mathrm{mg} / \mathrm{m}^{3} \text { for } \\
\text { slate cutters, } \\
24.70 \mathrm{mg} / \mathrm{m}^{3} \\
\text { general } \\
\text { environment ( } 7.29 \\
\text { years for cutters, } \\
7.62 \text { years for } \\
\text { non-cutters male } \\
\text { and } 14.69 \text { for non- } \\
\text { cutters female) }\end{array}$ & $\begin{array}{l}54.6 \% \text { surveyed showed } \\
\text { radiologic evidence of silicosis, } \\
\text { incidence and severity of fibrosis } \\
\text { correlated with degree and } \\
\text { duration of exposure and was } \\
\text { worse in females than males of } \\
\text { equal exposure levels }\end{array}$ \\
\hline
\end{tabular}




\begin{tabular}{|c|c|c|c|c|}
\hline $\begin{array}{l}\text { Medical } \\
\text { Survey }\end{array}$ & Iron Foundry & 188 & $\begin{array}{l}\text { Up to } 30 \text { times the } \\
\text { TLV for } \\
\text { coremakers } \\
\text { Up to } 9 \text { times the } \\
\text { TLV for fettlers } \\
(8.6)\end{array}$ & $\begin{array}{l}\text { Incidence of silicosis in } 9.6 \% \text { of } \\
\text { workers surveyed with } 1 \% \text { PMF, } \\
\text { prevalence correlated to } \\
\text { level/duration of exposure but not } \\
\text { with cigarette smoking. Smoking } \\
\text { did increase respiratory } \\
\text { symptomology, however. }\end{array}$ \\
\hline $\begin{array}{l}\text { Medical } \\
\text { Survey }\end{array}$ & Granite Workers & 320 & $\begin{array}{c}2.1 \mathrm{mg} / \mathrm{m}^{3} \text { for } \\
\text { silicotics } \\
0.95 \mathrm{mg} / \mathrm{m}^{3} \text { for } \\
\text { non silicotics } \\
\text { (at least one year) }\end{array}$ & $\begin{array}{l}\text { Chronic simple silicosis is } \\
\text { correlated to loss of lung function } \\
\text { attributable to fibrosis, lung } \\
\text { function losses correlated to } \\
\text { exposure to respirable silica } \\
\text { though declination in function may } \\
\text { also arise from larger sized mixed } \\
\text { particles }\end{array}$ \\
\hline $\begin{array}{l}\text { Medical } \\
\text { Survey }\end{array}$ & $\begin{array}{c}\text { Open-cast Coal } \\
\text { Mining }\end{array}$ & 1,249 & $\begin{array}{l}\leq 1.0 \mathrm{mg} / \mathrm{m}^{3} \\
\text { geometric mean } \\
\text { for groups, some } \\
\text { individual } \\
\text { measures were } \\
\text { higher }(\mathrm{n} / \mathrm{a})\end{array}$ & $\begin{array}{l}\text { Workers performing dustiest jobs } \\
\text { such as rock drillers etc. } \\
\text { increases risk for radiological } \\
\text { findings consistent with } \\
\text { pneumoconiosis, most likely } \\
\text { silicosis }\end{array}$ \\
\hline $\begin{array}{l}\text { Medical } \\
\text { Survey }\end{array}$ & Stone Carving & 42 & $\begin{array}{c}1.5 \mathrm{mg} / \mathrm{m}^{3}(15.5 \\
\text { years })\end{array}$ & $\begin{array}{l}\text { High prevalence of silica dust } \\
\text { correlated with increased } \\
\text { incidence of silicosis and reduced } \\
\text { lung function, duration of } \\
\text { exposure also contributed to } \\
\text { severity of silicosis }\end{array}$ \\
\hline
\end{tabular}




\begin{tabular}{|c|c|c|c|c|c|}
\hline $\begin{array}{l}\text { Medical } \\
\text { Survey }\end{array}$ & Sand Blasting & 75 & $\mathrm{n} / \mathrm{a}$ (14.2 years) & $\begin{array}{l}\text { Prevalence of progressive } \\
\text { massive fibrosis among silicotics } \\
\text { was } 39.7 \% \text {, high coincidence with } \\
\text { tuberculosis and calcified lymph } \\
\text { nodes also indicated as possible } \\
\text { contributors to transition from } \\
\text { silicosis to PMF }\end{array}$ & [428] \\
\hline $\begin{array}{l}\text { Medical } \\
\text { Survey }\end{array}$ & $\begin{array}{c}\text { Denim } \\
\text { Sandblasting }\end{array}$ & 60 & $\begin{array}{c}\mathrm{n} / \mathrm{a}(18.5 \text { months }) \\
\text { but, }(21.4 \text { months } \\
\text { for those with } \\
\text { silica) }\end{array}$ & $\begin{array}{l}73.3 \% \text { of patients had silicosis, all } \\
\text { cases were accelerated with } \\
11.4 \% \text { having PMF, clinical score } \\
\text { was positively correlated with } \\
\text { duration of exposure, latency } \\
\text { since exposure, and occurrence } \\
\text { of PMF }\end{array}$ & [429] \\
\hline $\begin{array}{l}\text { Medical } \\
\text { Survey }\end{array}$ & $\begin{array}{c}\text { Denim } \\
\text { Sandblasting }\end{array}$ & 32 & n/a (45 months) & $\begin{array}{l}\text { All silicosis cases began after } \\
\text { acute and relatively short-term } \\
\text { exposure ( } \leq 2 \text { years) to silica, } \\
19 \% \text { of patients died of PMF, } \\
\text { mean age of mortality was } 35 \\
\text { years suggesting over-exposure } \\
\text { to large amounts of silica }\end{array}$ & [430] \\
\hline
\end{tabular}




\begin{tabular}{|c|c|c|c|c|}
\hline $\begin{array}{l}\text { Medical } \\
\text { Survey }\end{array}$ & $\begin{array}{l}\text { Surface Coal } \\
\text { Mining }\end{array}$ & 2,328 & 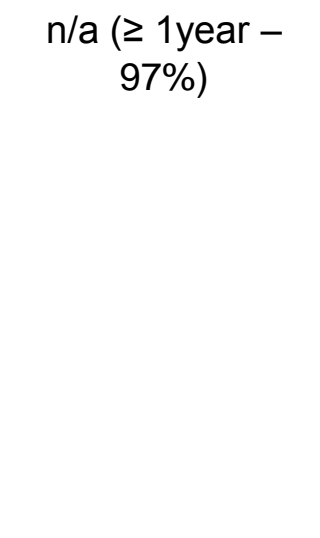 & $\begin{array}{l}2 \% \text { of patients exhibited } \\
\text { pneumoconiosis, with } 0.5 \% \\
\text { exhibiting signs of PMF, many of } \\
\text { which had no underground mining } \\
\text { history, } 37 \% \text { of those with } \\
\text { pneumoconiosis were shown to } \\
\text { have radiological findings } \\
\text { consistent with silicosis, incidence } \\
\text { of these findings was increased } \\
\text { among miners from Central } \\
\text { Appalachian regions }\end{array}$ \\
\hline $\begin{array}{l}\text { Medical } \\
\text { Survey }\end{array}$ & $\begin{array}{c}\text { Silica } \\
\text { Processing }\end{array}$ & 250 & $\begin{array}{c}\mathrm{n} / \mathrm{a}(13 / 14 \\
\text { silicotics had less } \\
\text { than } 10 \text { years of } \\
\text { tenure })\end{array}$ & $\begin{array}{l}\text { Of } 250 \text { employees surveyed } 14 \text { or } \\
5.6 \% \text { had radiologic findings } \\
\text { consistent with silicosis, all but } 1 \\
\text { had tenure of less than } 10 \text { years } \\
\text { suggesting accelerated silicosis. } 1 \\
\text { worker had PMF, and } 5 \text { silicotic } \\
\text { workers had findings consistent } \\
\text { with latent TB. }\end{array}$ \\
\hline Cohort & Coal Mining & 74 & $\mathrm{n} / \mathrm{a}(\mathrm{n} / \mathrm{a})$ & $\begin{array}{l}\text { Higher quartz content in the dust } \\
\text { of worker lungs resulted in more } \\
\text { severe lung lesions }\end{array}$ \\
\hline Cohort & Granite Workers & $\begin{array}{l}777 \text { granite } \\
\text { workers, } 122 \\
\text { controls }\end{array}$ & $\mathrm{n} / \mathrm{a}(\mathrm{n} / \mathrm{a})$ & $\begin{array}{l}\text { Highest prevalence of fibrosis in } \\
\text { dressing workers, drillers, and } \\
\text { sand blasters }\end{array}$ \\
\hline Cohort & Tungsten Mining & 1,151 & $\begin{array}{l}0.25 \mathrm{mg} / \mathrm{m}^{3} \text { and } \\
2.25 \mathrm{mg} / \mathrm{m}^{3}(\mathrm{n} / \mathrm{a})\end{array}$ & $\begin{array}{l}\text { Cumulative exposure to silica dust } \\
\text { in tungsten miners was positively } \\
\text { correlated with probability of } \\
\text { silicosis development }\end{array}$ \\
\hline
\end{tabular}




\begin{tabular}{|c|c|c|c|c|}
\hline Cohort & Construction & 1,056 & $\mathrm{n} / \mathrm{a}(20-25$ years $)$ & $\begin{array}{l}\text { Chronic simple silicosis correlated } \\
\text { to } 20+\text { years of exposure to silica } \\
\text { in the workplace, stone-splitters } \\
\text { and caisson workers at highest } \\
\text { risk, other coinciding } \\
\text { complications included TB }\end{array}$ \\
\hline Cohort & Coal Mining & 90,973 & $\mathrm{n} / \mathrm{a}(\mathrm{n} / \mathrm{a})$ & $\begin{array}{l}\text { Increases in radiological findings } \\
\text { associated with silica exposure, } \\
\text { pneumoconiosis, and PMF } \\
\text { occurred during } 2000-2008 \\
\text { compared with } 80 \text { s and } 90 \text { s } \\
\text { suggesting coal miner exposure to } \\
\text { respirable silica is too high }\end{array}$ \\
\hline Cohort & $\begin{array}{c}\text { Denim } \\
\text { Sandblasting }\end{array}$ & 145 & n/a (40+ months) & $\begin{array}{l}\text { Despite short exposure and } \\
\text { latency, incidence of silicosis and } \\
\text { progression of silicosis increased } \\
\text { in sandblaster previously } \\
\text { evaluated in } 2007 \text {. Of those } \\
\text { surveyed only } 1 \text { patient of those } \\
\text { surviving did not have progression } \\
\text { of disease. }\end{array}$ \\
\hline
\end{tabular}


Cohort Coal Mining

Post-

Granite

Mortem Quarrying

Post- Coal Mining

Mortem

Post-

Gold Mining

Mortem
$16,082 \mu \mathrm{g} / \mathrm{m}^{3}-\mathrm{y}$ lifetime mean (19.4 years)

n/a (21 years)

n/a (25-30 years)
All but 1 miner in 13 surveyed for rapid progressive pneumoconiosis had PMF. Only 4 of 13

demonstrated classic coal worker's pneumoconiosis, while 11 of 13 demonstrated mixed pneumoconiosis and lesions consistent with silicosis. Two of 13 displayed alveolar proteinosis and interstitial inflammation along with silicotic nodules indicating accelerated silicosis. Findings suggest exposure to coal dust containing high amounts of silica and silicates.

Fibrosis, evidence of pneumoconiosis in the absence of radiologic findings, consistent with 1955 exposure standard of 50 $\mu \mathrm{g} / \mathrm{m}^{3}$

Degree of lung fibrosis was highly correlated with silica lung content while emphysema was positively correlated with lung coal content

Lung quartz dust concentration was associated with severity of silicosis, though duration of exposure did not necessarily correlate with lung particle burden 


\begin{tabular}{|c|c|c|c|c|}
\hline $\begin{array}{l}\text { Post- } \\
\text { Mortem }\end{array}$ & $\begin{array}{l}\text { Agricultural } \\
\text { Workers }\end{array}$ & 112 & $\begin{array}{c}\text { Total dust for the } \\
\text { region } \mathrm{PM}_{10} \text { range } \\
43.5-122 \mu \mathrm{g} / \mathrm{m}^{3} \\
(\mathrm{n} / \mathrm{a})\end{array}$ & $\begin{array}{l}\text { Higher prevalence of } \\
\text { pneumoconiosis and small airway } \\
\text { disease was observed in } \\
\text { farmworkers compared with non- } \\
\text { farmworkers of the same region, } \\
\text { airway lesions were strongly } \\
\text { associated with mineral dust, } \\
\text { crystalline silica was significantly } \\
\text { associated with small airway } \\
\text { fibrosis }\end{array}$ \\
\hline $\begin{array}{l}\text { Post- } \\
\text { Mortem }\end{array}$ & Platinum Mining & 30 & n/a ( $\geq 1$ year) & $\begin{array}{l}\text { Levels of silica that platinum } \\
\text { miners are exposed to are high } \\
\text { enough to cause silicosis, } 25 \\
\text { workers surveyed had fibrotic } \\
\text { nodules in the lymph nodes, and } 5 \\
\text { had pulmonary silicosis. }\end{array}$ \\
\hline
\end{tabular}


Table 2: Retrospective Studies of Occupational Exposure to Diesel Exhaust with regard to Lung Cancer

\begin{tabular}{|c|c|c|c|c|c|c|}
\hline $\begin{array}{l}\text { Study } \\
\text { Design }\end{array}$ & $\begin{array}{l}\text { Occupation } \\
\text { Examined }\end{array}$ & $\begin{array}{l}\text { Participant } \\
\text { Number (n) }\end{array}$ & $\begin{array}{l}\text { Exposure Level } \\
\text { (Average Time) }\end{array}$ & $\begin{array}{l}\text { Latency } \\
\text { Period }\end{array}$ & Findings & Citation \\
\hline $\begin{array}{l}\text { Case } \\
\text { Study }\end{array}$ & Varied & $\begin{array}{c}2,291 \text { lung } \\
\text { cancer, } 2,570 \\
\text { controls }\end{array}$ & n/a (10+ years) & $\mathrm{n} / \mathrm{a}$ & $\begin{array}{l}\text { An increased risk for lung } \\
\text { cancer mortality was } \\
\text { found for truck drivers a } \\
\text { finding that could not be } \\
\text { accounted for by cigarette } \\
\text { smoking or additional } \\
\text { occupational exposure. }\end{array}$ & {$[444]$} \\
\hline $\begin{array}{l}\text { Case } \\
\text { Study }\end{array}$ & Varied & $\begin{array}{c}996 \text { cases, } \\
1085 \text { controls }\end{array}$ & $\begin{array}{c}\mathrm{n} / \mathrm{a}(\sim 25-30 \\
\text { years })\end{array}$ & $\mathrm{n} / \mathrm{a}$ & $\begin{array}{c}\text { Data suggest an } \\
\text { increased risk for lung } \\
\text { cancer mortality in long- } \\
\text { haul truckers and diesel } \\
\text { truck drivers however } \\
\text { quantifiable data on DE } \\
\text { exposure was not } \\
\text { available. }\end{array}$ & [445] \\
\hline $\begin{array}{l}\text { Case } \\
\text { Study }\end{array}$ & $\begin{array}{c}\text { Professional } \\
\text { Driving }\end{array}$ & $\mathrm{n} / \mathrm{a}$ & N/a $(n / a)$ & $\mathrm{n} / \mathrm{a}$ & $\begin{array}{l}\text { Excess risk for lung } \\
\text { cancer in chauffeurs } \\
\text { exposed to long-term DE } \\
\text { fumes was found even } \\
\text { after adjusting for } \\
\text { smoking history. }\end{array}$ & [446] \\
\hline $\begin{array}{l}\text { Case } \\
\text { Study }\end{array}$ & Varied & $\mathrm{n} / \mathrm{a}$ & $\begin{array}{l}\text { Estimated into } \\
\text { low-high } \\
\text { quartiles (n/a) }\end{array}$ & $\mathrm{n} / \mathrm{a}$ & $\begin{array}{l}\text { Men were at a higher risk } \\
\text { for incidence of lung } \\
\text { cancer, especially } \\
\text { squamous cell } \\
\text { carcinomas. This risk } \\
\text { increased with increasing }\end{array}$ & [447] \\
\hline
\end{tabular}




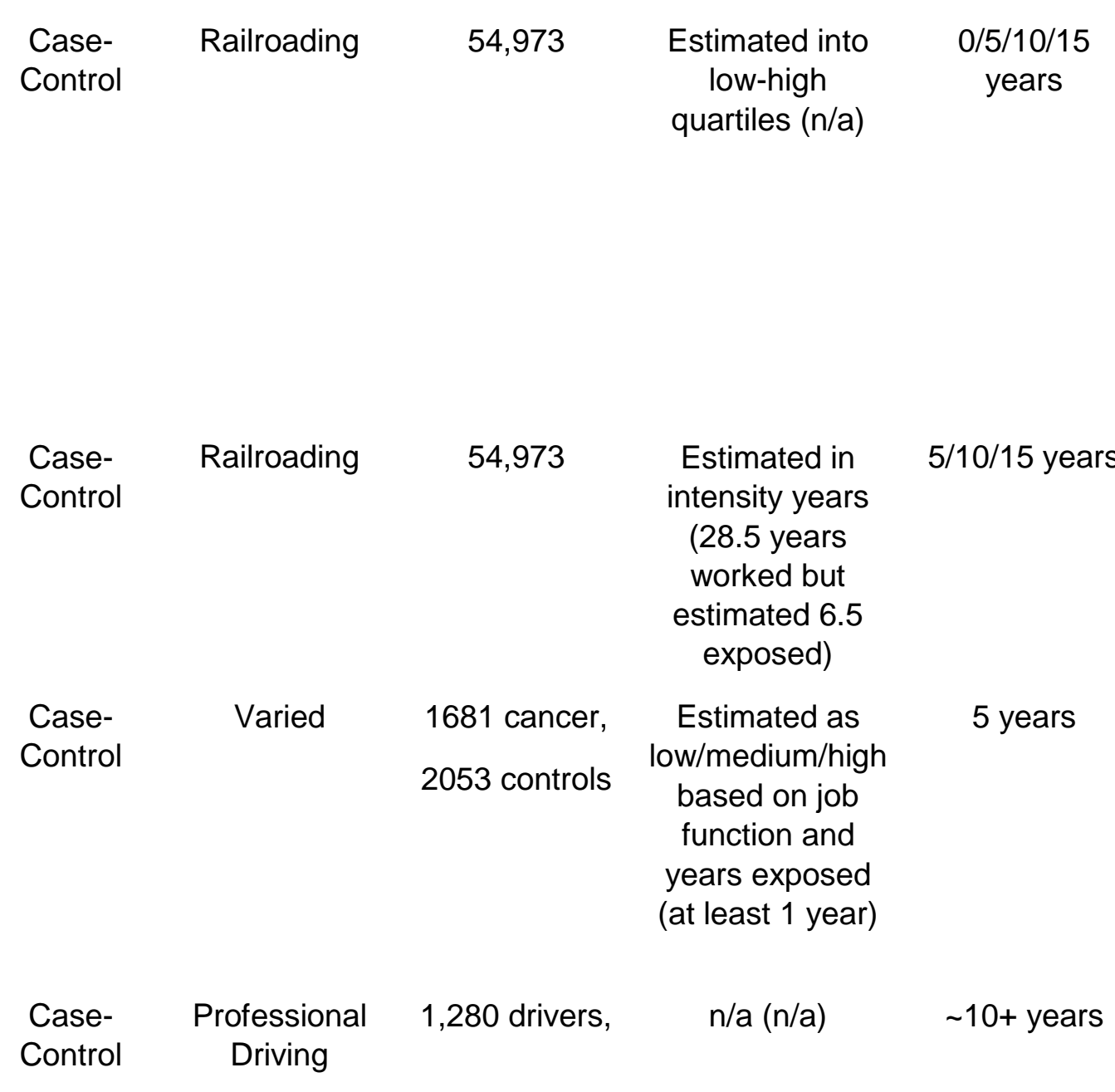

estimated intensity of exposure. Risk for other malignancies were found but to a lesser and in some case non-significant extent.

Operating a locomotive was associated with increased risk of lung cancer mortality but this risk did not increase with working tenure. Lung cancer mortality was increased on trains with diesel-powered locomotives.

Increased risk of dying from lung cancer compared with non-DE exposed jobs but not with increasing estimated exposure.

Excess risk of lung cancer was determined for truck drivers, taxi drivers, and railway conductors especially for squamous and large cell carcinomas

Professional drivers in Korea are exposed to 
33,924

general

population

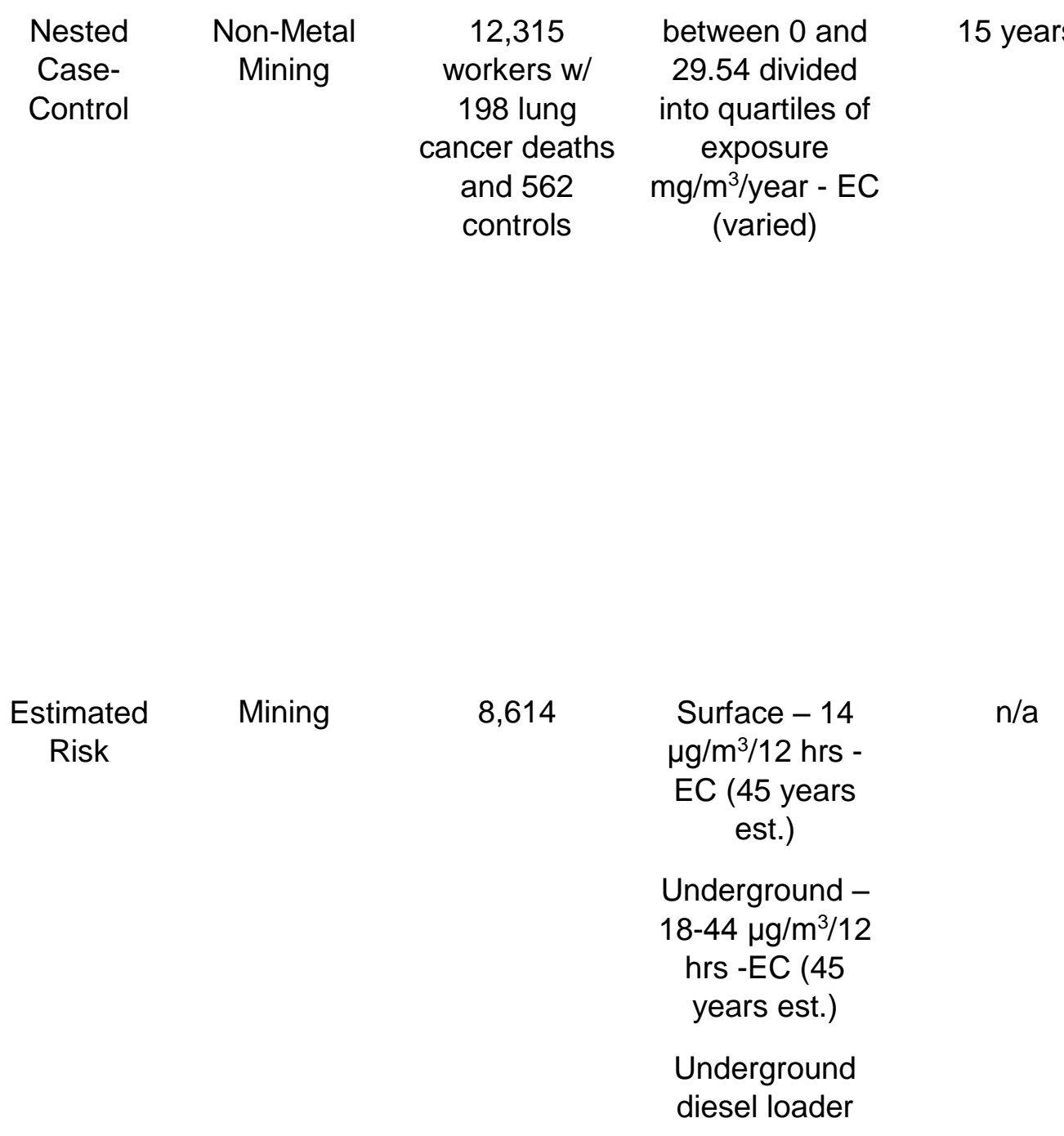

increased levels of DE and air pollution, which increases risk for incidence of lung cancer.

In a study of over 12,000 miners, historical

exposure to respirable EC was estimated. It was reported that an increased risk of dying from lung cancer was associated with exposureresponse relationship to DE. There was in increased trend in risk with increasing exposure to DE. This was regardless of facilities, noting that in some facilities smoking was prohibited.

Based on published risk it was determined that miners in Australia are exposed to levels of DE that would result in excess death of lung cancer and that additional controls for worker exposure are necessary. 
operators - 59

$\mu \mathrm{g} / \mathrm{m}^{3} / 12 \mathrm{hrs}-$

EC (45 years

est.)

\begin{tabular}{|c|c|c|c|c|c|}
\hline $\begin{array}{l}\text { Pooled } \\
\text { Analysis }\end{array}$ & Varied & $\begin{array}{c}13,304 \text { cases, } \\
16,282 \\
\text { controls }\end{array}$ & $\begin{array}{l}\text { Estimated into } \\
\text { low-high } \\
\text { quartiles (1-35 } \\
\text { years) }\end{array}$ & $n / a$ & $\begin{array}{l}\text { Occupational exposure } \\
\text { was consistently } \\
\text { associated with small } \\
\text { increase in risk for lung } \\
\text { cancer and there was a } \\
\text { significant exposure- } \\
\text { response trend. }\end{array}$ \\
\hline $\begin{array}{l}\text { Pooled } \\
\text { Analysis }\end{array}$ & Varied & $\begin{array}{l}1,593 \text { cases, } \\
1,427 \text { controls }\end{array}$ & $\begin{array}{l}\text { Estimated in } \\
\text { low-high relative } \\
\text { cumulative } \\
\text { exposure } \\
\text { (estimated 1- } \\
20+\text { ) }\end{array}$ & $\mathrm{n} / \mathrm{a}$ & $\begin{array}{l}\text { Both studies pooled } \\
\text { found an increased risk } \\
\text { for lung cancer } \\
\text { associated with exposure } \\
\text { to DE. Risk was higher for } \\
\text { squamous cell carcinoma } \\
\text { than for small cell or } \\
\text { adenocarcinomas. }\end{array}$ \\
\hline $\begin{array}{l}\text { Meta- } \\
\text { Analysis }\end{array}$ & Varied & $\begin{array}{l}\text { Pooled } 39 \\
\text { studies }\end{array}$ & $\mathrm{n} / \mathrm{a}(\mathrm{n} / \mathrm{a})$ & $10+$ years & $\begin{array}{c}\text { Epidemiological evidence } \\
\text { suggests that } \\
\text { occupational exposure to } \\
\text { DE has a causal } \\
\text { relationship with lung } \\
\text { cancer. }\end{array}$ \\
\hline $\begin{array}{l}\text { Meta- } \\
\text { Analysis }\end{array}$ & $\begin{array}{c}\text { Professional } \\
\text { Driving }\end{array}$ & $\begin{array}{l}\text { Pooled } 19 \\
\text { studies }\end{array}$ & $\mathrm{n} / \mathrm{a}(\sim 10+$ years $)$ & $\mathrm{n} / \mathrm{a}$ & $\begin{array}{l}\text { After adjusting for } \\
\text { smoking, this study } \\
\text { reported that professional } \\
\text { drivers are at an } 18 \%\end{array}$ \\
\hline
\end{tabular}

[452] 


\begin{tabular}{|c|c|c|c|c|c|}
\hline & & & & & $\begin{array}{c}\text { increased risk for } \\
\text { developing lung cancer. }\end{array}$ \\
\hline Cohort & Dock Working & 6,071 & $\mathrm{n} / \mathrm{a}(\mathrm{n} / \mathrm{a})$ & $(\mathrm{n} / \mathrm{a})$ & $\begin{array}{l}\text { A tendency for increased } \\
\text { risk of mortality due to } \\
\text { lung cancer was found; } \\
\text { however, smoking related } \\
\text { causalities could not be } \\
\text { excluded. }\end{array}$ \\
\hline Cohort & Varied & $\begin{array}{c}62,800 \\
\text { exposed, } \\
307,143 \\
\text { unexposed }\end{array}$ & $\mathrm{n} / \mathrm{a}(\mathrm{n} / \mathrm{a})$ & $(\mathrm{n} / \mathrm{a})$ & $\begin{array}{l}\text { An increased risk for } \\
\text { mortality due to lung } \\
\text { cancer was found even } \\
\text { after adjusting for } \\
\text { smoking status. } \\
\text { Occupations that were } \\
\text { significantly associated } \\
\text { with increased risk } \\
\text { included miners and } \\
\text { heavy equipment } \\
\text { operators, while truckers } \\
\text { and railroaders had a } \\
\text { weaker association. }\end{array}$ \\
\hline Cohort & $\begin{array}{l}\text { Bus and Tram } \\
\text { Driving }\end{array}$ & 18,174 & $\mathrm{n} / \mathrm{a}(\sim 10$ years $)$ & $\begin{array}{c}\text { Greater than } \\
15 \text { years } \\
\text { positively } \\
\text { correlated }\end{array}$ & $\begin{array}{l}\text { Increased incidence of all } \\
\text { malignancies. For people } \\
\text { employed longer than } 3 \\
\text { months risk of lung } \\
\text { cancer incidence was } \\
\text { significant for men and } \\
\text { women. Smoking cannot } \\
\text { be ruled out as a } \\
\text { confounder. }\end{array}$ \\
\hline
\end{tabular}




\begin{tabular}{|c|c|c|c|c|c|}
\hline Cohort & Potash Mining & 5,536 & $\begin{array}{c}2.70 \mathrm{ymg} / \mathrm{m}^{3}(15 \\
\text { years })\end{array}$ & 19 years & $\begin{array}{l}\text { Relative risk of lung } \\
\text { cancer was doubled for } \\
\text { miners exposed to the } \\
\text { highest measure of Total } \\
\text { carbon after }>20 \text { years of } \\
\text { exposure, however, the } \\
\text { elevation was still not } \\
\text { considered to be } \\
\text { significant. Smoking was } \\
\text { not considered a } \\
\text { confounder in this study, } \\
\text { but cannot be ruled out. }\end{array}$ \\
\hline Cohort & $\begin{array}{l}\text { Heavy } \\
\text { Equipment } \\
\text { and Truck } \\
\text { Driving }\end{array}$ & $\begin{array}{c}20,728, \\
119,984 \\
\text { referants }\end{array}$ & $\mathrm{n} / \mathrm{a}(\mathrm{n} / \mathrm{a})$ & $\mathrm{n} / \mathrm{a}$ & $\begin{array}{l}\text { Increased risk of lung } \\
\text { cancer for truck drivers } \\
\text { exposed to DE was } \\
\text { found, but not for HEO. } \\
\text { Increased risk for prostate } \\
\text { cancer was also found for } \\
\text { truck drivers. }\end{array}$ \\
\hline Cohort & Truck Driving & 54,319 & n/a ( 20 years) & $\mathrm{n} / \mathrm{a}$ & $\begin{array}{l}\text { Among the US working } \\
\text { population examined, } \\
\text { increased mortality due to } \\
\text { lung cancer was } \\
\text { observed for all drivers } \\
\text { and dock workers. } \\
\text { Relative risk for lung } \\
\text { cancer was found to be } \\
\text { consistent with other } \\
\text { occupational studies. } \\
\text { Elevated risk for lung } \\
\text { cancer was identified for } \\
\text { workers in the US } \\
\text { Trucking Industry }\end{array}$ \\
\hline
\end{tabular}


Cohort Truck Driving

31,135

n/a ( 20 years)

$\mathrm{n} / \mathrm{a}$ especially for drivers;

however, smoking and

lifestyle may play a role in these health risks.

Regular exposure to DE and other MVE have an elevated risk of lung cancer, which was associated with working tenure. 


\section{Chapter 2 - Study 1}

Published in: Inhalation Toxicology

Inhal Toxicol. 2017 Jun;29(7):322-339. 


\title{
Pulmonary Toxicity Following Acute Co-Exposures to Diesel Particulate Matter and $\alpha$-Quartz Crystalline Silica in the Sprague-Dawley Rat
}

\author{
Breanne Y. Farris ${ }^{1,2}$, James M. Antonini ${ }^{1,2}$, Jeffrey S. Fedan ${ }^{1,2}$, Robert R. Mercer ${ }^{1}$, \\ Katherine A. Roach ${ }^{1,2}$, Bean T. Chen ${ }^{1}$, Diane Schwegler-Berry ${ }^{1}$, Michael L. Kashon ${ }^{1}$, \\ Mark W. Barger ${ }^{1}$, and Jenny R. Roberts ${ }^{1,2}$. \\ ${ }^{1}$ National Institute for Occupational Safety and Health, Morgantown, WV \\ ${ }^{2}$ West Virginia University, School of Medicine, Morgantown, WV
}

\section{Article History}

Received 20 April 2017

Revised 15 June 2017

Accepted 26 July 2017

\section{Keywords}

Crystalline silica; diesel exhaust particulate matter; immune response; occupational exposure; pulmonary fibrosis 


\section{Abstract}

The effects of acute pulmonary co-exposures to silica and diesel particulate matter (DPM) that may occur in various mining operations were investigated in vivo. Rats were exposed by intratracheal instillation (IT) to silica (50 or $233 \mu \mathrm{g}$ ), DPM (7.89 or $50 \mu \mathrm{g}$ ), or silica and DPM combined in phosphate-buffered saline (PBS), or to PBS alone (control). At 1 day, 1 week, 1 month, 2 months, and 3 months post-exposure bronchoalveolar lavage and histopathology were performed to assess lung injury, inflammation, and immune response. While higher doses of silica caused inflammation and injury at all time-points, DPM exposure alone did not. DPM (50 $\mu \mathrm{g})$ combined with silica $(233 \mu \mathrm{g})$ increased inflammation at 1 week and 1 month post-exposure and caused an increase in the incidence of fibrosis at 1 month compared to exposure to silica alone. To assess susceptibility to lung infection following co-exposure, rats were exposed by IT to $233 \mu \mathrm{g}$ silica, $50 \mu \mathrm{g}$ DPM, a combination of the two, or PBS control 1 week before intratracheal inoculation with $5 \times 10^{5}$ Listeria monocytogenes. At 1, 3, 5, 7, and 14 days following infection, pulmonary immune response and bacterial clearance from the lung were evaluated. Co-exposure to DPM and silica did not alter bacterial clearance from the lung compared to control. Although DPM and silica co-exposure did not alter pulmonary susceptibility to infection in this model, the study showed that noninflammatory doses of DPM had the capacity to increase silica-induced lung injury, inflammation, and onset/incidence of fibrosis. 


\section{Introduction}

The natural gas and oil industry is one of the most rapidly growing industries in the United States. Specifically, the industry is expanding in the area of hydraulic fracturing, "fracking," or horizontal drilling. This process extracts natural resources from otherwise inaccessible deposits below the earth's surface, provided more than 2 million jobs in 2012, and is estimated to support approximately 4 million jobs by 2025 [460]. Two respirable particulates identified as possible exposure hazards in fracking workplaces include diesel particulate matter (DPM) and $\alpha$-quartz crystalline silica $\left(\mathrm{SiO}_{2}\right)$ [461]. These two particulates are also present in combination in other mining operations including below ground mining operations, particularly in coal extraction. Although toxicity of these two particulates have been studied in detail as individual exposures, there are currently no in vivo studies, as far as we are aware, that have examined the pulmonary effects of the particulates in combination at doses that are relevant to various mining operations.

In addition to underground mining operations and operations in hydraulic fracturing, where significant numbers of diesel engines are utilized (transport, drilling, and pumping), diesel exhaust (DE) exposure is also prevalent in industries including trucking and railroad operations [182]. DE is a highly complex mixture of chemical substances, but is primarily composed of an elemental carbon (EC) core having additional organic carbon constituents adsorbed to it. Other than the EC components, compounds typically contained in DE include: polyaromatic hydrocarbons (PAHs), sulfates, silicates, nitrates, as well as metallic particulates [182]. DPM exposure is linked to a variety of adverse health outcomes, including enhanced sensitization to allergic stimulus, development and aggravation of asthma, chronic bronchitis, decreased lung function, airway inflammation, decreased vascular function, and development of cancers, as reported in epidemiological studies [234, 410-415, 462]. Based on these studies, DE and DPM have been identified by the International Agency for Cancer Research (IARC) as a group 1 carcinogen [182, 463]. In addition to human cohort studies, studies involving healthy human volunteers exposed to DE/DPM in a controlled chamber have also shown that DE/DPM caused pulmonary inflammation in acute exposure scenarios [234, 464]. Controlled human studies as reviewed by Ghio et al. 
$[465,466]$ show that inflammation occurs in the respiratory tract of humans exposed to DPM and that this occurs in a dose-dependent manner. DPM toxicity has also been investigated in vivo and in vitro. DPM has been shown to enhance airway reactivity, inflammation, production of reactive oxygen species (ROS), and increased development and severity of allergic asthma, lung fibrosis, lung injury, alveolar edema, lung cancer and mortality in exposed animals [289, 308, 327, 467-473]. Studies conducted in vitro suggest, similarly, that DPM contributes to airway inflammation, production of reactive oxidants, unfolded protein response, allergic response, decreased mucociliary clearance, and upregulation of cancer-associated proteins in lung cells [333, 377, 380, 467, 474-479]. Furthermore, DPM has been shown to alter the pulmonary immune function and is associated with increased susceptibility to infection [316, 327, 480-485].

Regulations to control occupational exposure to DE or DPM are recognized in several countries, and regulations vary relative to underground mining operations versus above-ground mining and non-mining workplaces. In addition, exposure is differentially regulated by DE constituents (total particle, elemental carbon, nitrogen) [179-182]. In the United States, recommended exposure limits (REL) exist for many of the components of diesel exhaust as individual emissions; however, there is currently no Occupational Safety and Health Administration (OSHA) regulation of DPM in nonmining, occupational settings. The Mine Safety and Health Administration (MSHA) REL for DE and DPM in underground mine operations is a time-weighted average (TWA), based on an 8-hour work day, of $160 \mu \mathrm{g} / \mathrm{m}^{3}$ total carbon, which includes DPM as elemental carbon. Additionally, The California Department of Health Services (CDHS) has recommended an occupational exposure limit (OEL) of $20 \mu \mathrm{g} / \mathrm{m}^{3}$ elemental carbon [184]. Both Switzerland and Australia have set OELs at $100 \mu \mathrm{g} / \mathrm{m}^{3}$ elemental carbon $[179,183]$.

Workplace exposure to $\mathrm{SiO}_{2}$ also occurs in a multitude of occupations and are well documented for a variety of mining operations, as well as in masonry, painting, sand blasting, construction, demolition, and ceramics [404, 405]. $\mathrm{SiO}_{2}$ is one of the most common minerals occurring in the earth's crust and is a major component of sand. Sand that contains $\mathrm{SiO}_{2}$ is the primary proppant, or substance used to stabilize the fracture 
during hydraulic fracturing operations and subsequent resource extraction. A respirable fraction of this sand can become airborne when transported onto/around fracking sites or pumped at high volumes/pressure into the well-bore [486]. The respiratory toxicity of the crystalline form of $\mathrm{SiO}_{2}$ is well-established, and therefore OSHA requires workplaces to follow the permissible exposure limits (PEL) set at $0.05 \mathrm{mg} / \mathrm{m}^{3}$ as a TWA for respirable crystalline $\mathrm{SiO}_{2}$. Inhalation of respirable $\mathrm{SiO}_{2}$ has been shown to cause lung inflammation and injury, chronic obstructive pulmonary disorders, bronchitis, emphysema, fibrosis, silicotic pneumoconiosis, cancer, heart disease, autoimmune disorders, and increased risk for infection in humans [18, 35, 404, 406-409], depending upon duration and level of exposure.

Further, in vivo studies of $\mathrm{SiO}_{2}$ exposure have investigated the mechanisms associated with pathologies, such as fibrosis and cancer, including: chronic inflammation and the up-regulation of acute inflammatory mediators like leukotriene $B_{4}$, up-regulation of the NF-kB pathway, increased oxidant burden attributable to both the presence of free radicals associated with particles and oxidant production by cells in response to $\mathrm{SiO}_{2}$ exposure, up-regulation of genes associated with mucus production and pulmonary toxicity, and activation of the inflammasome [80, 123, 128, 487-489]. In vitro studies have shown production of oxidants and inflammatory mediators, activation of cell-signaling pathways associated with cancer, damage to DNA, and increased expression of genes related to inflammation, apoptosis, cancer, and fibrosis in response to $\mathrm{SiO}_{2}$ exposure [490-495], furthering support for mechanisms underlying the pathologies observed in animal models of disease related to occupational exposure. $\mathrm{SiO}_{2}$ exposure has also been implicated in the alteration to the immunological environment in the respiratory tract $[91,496]$ and contributions to respiratory susceptibility to some infections [158, 497].

While the pulmonary toxicity to constituents of diesel, and $\mathrm{SiO}_{2}$ are well-studied as individual exposures, little is known about the effects these particles may have when encountered as a mixed exposure. Mining operations including hydraulic fracturing for oil and gas and above and below ground metal and non-metal mining operations, are environments where workers may be exposed to constituents of diesel and $\mathrm{SiO}_{2}$ 
simultaneously, rendering it paramount to establish whether co-exposure to these particles will enhance or alter pulmonary toxicity. The current studies were designed to investigate the hypothesis that combined exposures to these particles would result in amplified injury, inflammation, and cellular recruitment to the lung in an acute exposure scenario. Additionally, the studies addressed whether or not cell-mediated clearance of a pathogen would be altered by the co-exposure. Existing in vivo and in vitro studies documenting the toxicity of DPM and $\mathrm{SiO}_{2}$ in vivo often examine doses of these materials representative of chronic exposures or doses that exceed typical workplace exposures in single bolus doses [84, 324, 498-501]. Therefore, doses for these studies were based on field measurements of $\mathrm{SiO}_{2}$ taken during active above-ground mining operations [486]. Values for diesel were derived from both underground mining values [502] for high dose values and from existing OELs for elemental carbon for the lower dose [181, 183, 503]. A single acute bolus dose of silica, diesel particulate, or a combination of the particles at different doses were administered to Sprague-Dawley rats by intratracheal instillation. Lung injury, inflammation, and immune responses were evaluated over a three-month time-course. Additionally, alteration in susceptibility to pulmonary infection following exposure to the particles in combination was evaluated to further assess alterations in immune response.

\section{Methods}

Particles. Particles in these studies are used as surrogates to represent work site exposures. DPM was obtained from the National Institute of Standards and Technology (NIST) as a commercially available NIST Standard Reference Material (SRM) 2975 (referred to as DPM in the study). SRM 2975 was originally collected from an industrial forklift in the 1990s [504]. The surrogate particle used for $\mathrm{SiO}_{2}$ is $\alpha$-quartz crystalline silica (SIL; MIN-U-SIL® 5, US SILICA; Berkeley Springs, WV).

Dose Determination. Doses were derived from field measurements of particles and applied to the equation below (EQ1) to quantify the amount of particle that would deposit in the lung of an average male worker doing moderate work for 12 hours per day for a period of 14 continuous days using a pulmonary deposition efficacy for each particle derived using a software-based algorithm that considers particle characteristics 
including shape and density [505]. These doses were then normalized to the surface area of both the worker's and the rat's lungs to obtain doses for in vivo studies (EQ2). DPM estimated as elemental carbon was reported to be variable depending on operations at hydraulic fracturing sites; (personal communication with JS related to Esswein et al., 2013; unreferenced) however, it was generally below the MSHA recommendation of $160 \mu \mathrm{g} / \mathrm{m}^{3}$ total carbon ( $120 \mu \mathrm{g} / \mathrm{m}^{3}$ elemental carbon equivalent), and DPM in underground mining operations has been measured in excess of $500 \mu \mathrm{g} / \mathrm{m}^{3}$ as elemental carbon as reviewed by Pronk et al. (2009). Therefore, in this study, two doses of DPM were evaluated. The low dose $(7.89 \mu \mathrm{g}$ per rat) was derived from the OELs for DPM measured as elemental carbon of $100 \mu \mathrm{g} / \mathrm{m}^{3}$ TWA $(0.100 \mu \mathrm{g} / \mathrm{l})$ (EQ3).The high dose of DPM was derived from high elemental carbon level measurements taken during underground mining of $637 \mu \mathrm{g} / \mathrm{m}^{3}(0.637 \mu \mathrm{g} / \mathrm{l})(\mathrm{EQ} 4)$ [506]. The $\mathrm{SiO}_{2}$ high dose (233 $\left.\mu \mathrm{g} \mathrm{SIL}\right)$ was derived from the highest $\mathrm{SiO}_{2}$ levels measured during hydraulic fracturing site visits [486], $2.5 \mathrm{mg} / \mathrm{m}^{3}(2.5 \mu \mathrm{g} / \mathrm{l})(\mathrm{EQ} 5)$. In addition, to assess exposure to equivalent doses of both particles at a less toxic dose of SIL, $50 \mu \mathrm{g}$ of SIL was also evaluated as the low dose of SIL.

\section{EQ1 - Basic equation for standard worker lung deposition:}

$=($ field concentration, $\mu \mathrm{g} / \mathrm{l}) \times($ minute ventilation, $\mathrm{l} / \mathrm{min}) \times$ (exposure time per day, $\min / \mathrm{d}) \times$ (total fracking days, $\mathrm{d}$ ) $\times$ (pulmonary deposition efficacy, unitless)

\section{EQ2 - Equation for normalization to rat lung surface area:}

$=($ worker's deposited dose/average male worker lung surface area) $\times$ (average rat lung surface area)

\section{EQ3 - Equation for low DPM dose:}

Worker lung deposition $=(0.10 \mu \mathrm{g} / \mathrm{l}) \times(20 \mathrm{l} / \mathrm{min}) \times(720 \mathrm{~min} /$ day $) \times(14$ days $) \times$ $(0.10)=2,016 \mu \mathrm{g}$

Dose calculated $=\left(2,016 \mu \mathrm{g} / 102.2 \mathrm{~m}^{2}\right) \times\left(0.4 \mathrm{~m}^{2}\right) \approx 7.89 \mu \mathrm{g}$ per rat EQ4 - Equation for high DPM dose: 
Worker lung deposition $=(0.637 \mu \mathrm{g} / \mathrm{l}) \times(20 \mathrm{l} / \mathrm{min}) \times(720 \mathrm{~min} /$ day $) \times(14$ days $) \times$ $(0.10) \approx 12,842 \mu \mathrm{g}$

Dose calculated $=\left(12,842 \mu \mathrm{g} / 102.2 \mathrm{~m}^{2}\right) \times\left(0.4 \mathrm{~m}^{2}\right) \approx 50 \mu \mathrm{g}$ per rat

\section{EQ5 - Equation for high SIL Dose:}

Worker lung deposition $=(2.5 \mu \mathrm{g} / \mathrm{I}) \times(20 \mathrm{l} / \mathrm{min}) \times(720 \mathrm{~min} /$ day $) \times(14$ days $) \times$ $(0.118)=59,472 \mu \mathrm{g}$ per worker

Dose calculated $=\left(59,472 \mu \mathrm{g} / 102.2 \mathrm{~m}^{2}\right) \times\left(0.4 \mathrm{~m}^{2}\right) \approx 233 \mu \mathrm{g}$ per rat

DPM and SIL Preparation and Characterization. Particles were prepared in USPgrade phosphate-buffered sterile saline (PBS) without $\mathrm{Ca}^{2+}$ or $\mathrm{Mg}^{2+}$ (AMERESCO; Solon, $\mathrm{OH}$ ) for intratracheal instillation. A sonication process was used to disperse agglomerated particles. DPM was prepared as a $2 \mathrm{mg} / \mathrm{ml}$ stock solution and SIL was prepared as a $10 \mathrm{mg} / \mathrm{ml}$ stock solution, vortex mixed for one min, followed by $1 \mathrm{~min}$ of continuous sonication (GE130PB ultrasonic processor; General Electric, Boston, MA). Aliquots of stock solution were then further diluted to: $7.89 \mu \mathrm{g}$ DPM, $50 \mu \mathrm{g}$ DPM, $50 \mu \mathrm{g}$ SIL, $233 \mu \mathrm{g}$ SIL, $7.89 \mu \mathrm{g}$ DPM + $233 \mu \mathrm{g}$ SIL , $50 \mu \mathrm{g}$ DPM + $50 \mu \mathrm{g} \mathrm{SIL} \mathrm{,} \mathrm{or} 50 \mu \mathrm{g}$ DPM + $233 \mu \mathrm{g}$ SIL in $0.3 \mathrm{ml}$ PBS. Particles were then vortex mixed for an additional $30 \mathrm{sec}$, followed by $15 \mathrm{sec}$ of sonication prior to intratracheal instillation. Particle preparations were dried onto filters and evaluated by field emission scanning electron microscopy (FESEM) to determine particle size. The average agglomerate size of the DPM was approximately $2 \mu \mathrm{m}$. SIL was evenly distributed in suspension with primary particle size of approximately $2 \mu \mathrm{m}$. Energy dispersive X-ray (EDX) was performed to determine the elemental components of the particles as a measure of particle purity. The DPM was comprised primarily of the carbonaceous core, or EC of diesel, with very little detectable trace elements including: Chromium, Copper, Iron, Nickel, Oxygen, Silicon, and Zinc. Due to their volatile nature, PAHs cannot be measured using EDX, but the total extractable mass including the certified mass fractions of PAHs contained in NIST SRM 2975 is given as $2.7 \%$ of the total sample as described in the certificate of analysis [504]. No trace metals were detected in the SIL sample. 
Animals. Male Sprague-Dawley [Hla:(SD) CVF] (SD) rats from Hilltop Lab Animals (Scottdale, PA), weighing approximately $300 \mathrm{~g}$ and free of viral pathogens, parasites, mycoplasmas, Helicobacter, and CAR Bacillus, were used for all studies. Rats were housed in the pathogen free, environmentally controlled, Association for Assessment and Accreditation of Laboratory Animal Care International (AAALAC)-accredited facility. The rats were housed in ventilated polycarbonate cages, two per cage, on Alpha-Dri cellulose chips and hardwood Beta chips as bedding; they were provided HEPA-filtered air, irradiated Teklad 2918 diet, and tap water ad libitum; and were allowed to acclimate for one week before exposure. Rats were not restricted from enrichment activity (i.e., chewing/climbing). All animal procedures used during the study were reviewed and approved by the Institutional Animal Care and Use Committee (IACUC).

Time-Course Study Design. In Vivo Lung Injury, Inflammation, and Disease. On day 0, rats were lightly anesthetized with an intraperitoneal injection of $30-40 \mathrm{mg} / \mathrm{kg}$ sodium methohexital (Brevital; Eli Lilly, Indianapolis, IN) and intratracheally instilled with $7.9 \mu \mathrm{g}$ DPM, $50 \mu \mathrm{g}$ DPM, $50 \mu \mathrm{g} \mathrm{SIL}, 233 \mu \mathrm{g}$ SIL, $7.89 \mu \mathrm{g}$ DPM + $233 \mu \mathrm{g}$ SIL, $50 \mu \mathrm{g}$ DPM + 233 $\mu \mathrm{g}$ SIL, $50 \mu \mathrm{g}$ DPM + $50 \mu \mathrm{g} \mathrm{SIL}$, or vehicle control (PBS). All particles were delivered in $0.3 \mathrm{ml}$ USP grade PBS. All animals were humanely euthanized at 1 day, 1 week, 1 month, 2 months, or 3 months following particle exposure ( $n=8 / \mathrm{group} /$ time point) with an intraperitoneal injection of $100-300 \mathrm{mg} / \mathrm{kg}$ sodium pentobarbital followed by exsanguination. The trachea was cannulated, bronchoalveolar lavage (BAL) was performed on the right lung lobes, and BAL cells (BALCs) and fluid (BALF) were retained for analysis of parameters indicative of inflammation, injury, oxidant production, and immune function. Lung injury and inflammation were evaluated as the presence of lactate dehydrogenase (LDH) activity, cytokines, and chemokines in BALF. BALCs were centrifuged onto slides, stained, and counted to determine cell phenotype differential. BAL lymphocytes were further differentiated by phenotype by flow cytometry. Lymphocytes from lymph nodes draining the lung were also differentiated by phenotype. Chemiluminescence assays were used to measure oxidant/free radical production by BAL phagocytes to evaluate the inflammatory response. The left lung was excised, pressure-inflated with 10\% neutral-buffered formalin, and weighed with waterdisplacement to determine fixed lung volume. Histopathological assessment of lung 
injury, inflammation, and disease were performed and morphometric analysis of fibrillar collagen content was conducted.

Infection Study Design. Pulmonary Immune Response to Infection Following Particle Exposure. In this study, rats were exposed to $50 \mu \mathrm{g}$ DPM, $233 \mu \mathrm{g} \mathrm{SIL,} 50 \mu \mathrm{g} \mathrm{DPM} \mathrm{+}$ $233 \mu \mathrm{g} \mathrm{SIL} \mathrm{combined} \mathrm{in} 0.3 \mathrm{ml}$ PBS, or PBS alone (vehicle control) by intratracheal instillation as described above. One week following particle exposure, rats were intratracheally instilled with $\sim 5 \times 10^{5}$ colony forming units (cfu) of Listeria monocytogenes (strain 10403S, serotype 1) in $0.5 \mathrm{ml}$ of sterile PBS. Animals were euthanized at $1,3,5,7$, and 14 days following infection ( $n=8 /$ dose/time point). BAL was performed on the right lung lobes, and BALC and BALF were retained for analysis of parameters indicative of inflammation, injury, and immune alteration as described for the time course study. The left lung was clamped during lavage and subsequently excised, homogenized, and cultured to evaluate bacterial clearance from the lung.

BAL. BAL was performed at each time point after exposure. Following euthanasia as described above, the trachea was cannulated, the chest cavity was opened, the left lung bronchus was clamped off, and BAL was performed on the right lung lobes. BAL was recovered in two fractions. The first fraction of the BAL was obtained by inflating the right lung with $4 \mathrm{ml}$ of PBS, massaging for $30 \mathrm{sec}$, withdrawing, and repeating the process a second time with the same $4 \mathrm{ml}$ of PBS. The second lavage fraction consisted of repeated $6 \mathrm{ml}$ volumes of PBS instilled with massaging of the chest cavity, withdrawal, and combination until a $30 \mathrm{ml}$ volume was recovered. For each animal, both fractions of BAL were centrifuged, the cell pellets were combined and re-suspended in $1 \mathrm{ml}$ of PBS, and the acellular fluid from the first fraction was retained for further analysis described for BALF below.

Analysis of LDH Activity. The level of LDH activity in the BALF of all treatment groups was measured at each time point after exposure to evaluate cytotoxicity as a measure of lung injury. Measurement of LDH activity in the acellular fluid was obtained using a Cobas Mira chemistry analyzer (Roche Diagnostic Systems; Montclair, IN). LDH activity was quantified by detection of the oxidation of lactate coupled to the reduction of NAD+ at a spectrophotometric setting of $340 \mathrm{~nm}$. 
BALF Protein Analysis. Cytokines and chemokines involved in inflammatory and immune responses were measured at each time point after exposure in the BALF of rats treated with PBS, DPM, SIL, or DPM/SIL combinations, with commercially-available enzyme-linked immunosorbent assay (ELISA) kits or by multiplex array. The following cytokines and chemokines were quantified by ELISA: tumor necrosis factor- $\alpha$ (TNF- $\alpha$ ), transforming growth factor- $\beta$ (TGF- $\beta$ ), interleukin (IL)-10, IL-12p70, monocyte chemotactic protein (MCP)-1, macrophage inflammatory protein (MIP)-2 (Novex, Life Technologies; Grand Island, New York); IL-2, IL-4, IL-6, interferon (IFN)-ү (R\&D Systems; Minneapolis, Minnesota); and osteopontin (OPN, Enzo Life Sciences; Farmingdale, New York). Additionally, two matrix metalloproteinases (MMP), MMP-2 and MMP-9, were quantified by ELISA, as well as tissue inhibitor of metalloproteinases (TIMP)-1 (R\&D Systems) as indices of tissue remodeling due to injury. IL-1 $\alpha$, IL-1 $\beta$, IL5, IL-13, IL-17A, IL-18, granulocyte colony stimulating factor (G-CSF), granulocyte and macrophage colony stimulating factor (GM-CSF), eotaxin, leptin, MIP-1a, epidermal growth factor (EGF), IFN-y inducible protein (IP)-10, growth-regulated oncogene/keratinocyte chemoattractant (GRO/KC), vascular endothelial growth factor (VEGF), fractalkine, lipopolysaccharide (LPS)-induced CXC chemokine (LIX) and regulated on activation, normal T-cell expressed and secreted (RANTES) were assessed by multiplex array (Eve Technologies; Alberta, CA).

BALC Differentials. Total BALC collected from rats intratracheally-instilled with PBS, DPM, SIL, or DPM + SIL in combination were counted using a Coulter Multisizer II (Coulter Electronics; Hialeah, FL). BALC differentials were performed to determine the total number of alveolar macrophages (AMs), neutrophils, lymphocytes, and eosinophils. Briefly, $5 \times 10^{4}$ cells from each rat were spun down onto slides with a Cytospin 3 centrifuge (Shandon Life Sciences International; Cheshire, England) and labeled with Hema $3 \AA$ stain (Fisher Scientific; Kalamazoo, MI) to differentiate cell types. Two hundred cells per slide were counted, and the percentage of AMs, neutrophils, lymphocytes, and eosinophils was multiplied by the total number of cells to calculate the total number of each cell type. 
Chemiluminescence (CL). To measure the production of reactive oxidant species by BALC, CL was measured according to the method of Antonini et al. [507]. Luminol (Sigma-Aldrich; St. Louis, MO) was used as an amplifier to enhance detection of the light, and $0.2 \mathrm{mg} / \mathrm{mL}$ of unopsonized zymosan (Sigma-Aldrich) or $3 \mu \mathrm{M}$ phorbol myristate acetate (PMA; Sigma-Aldrich) was added to the assay immediately prior to the measurement of $\mathrm{CL}$ to activate the cells. Because rat neutrophils do not respond to unopsonized zymosan, the zymosan-stimulated CL produced is from AMs, whereas the soluble stimulant, PMA, activates both neutrophils and AMs to generate reactive oxidant species [508]. CL was quantified using an automated Berthold Autolumat LB 953 luminometer (Wallace, Inc.; Gaithersburg, MD). Each sample was measured for $15 \mathrm{~min}$, and the integral of counts per minute (cpm) versus time was calculated. The production of $\mathrm{CL}$ was calculated as the $\mathrm{cpm}$ of stimulated cells minus the $\mathrm{cpm}$ of the corresponding resting cells, then normalized to the total number of BAL AMs for zymosan-stimulated $\mathrm{CL}$ and total BALC for PMA-stimulated CL.

\section{Phenotypic Quantification of BALC and Mediastinal Lymph Node (MLN) Cells by}

Flow Cytometry. Lymphocyte populations in BALC and MLN were differentially assessed for phenotype by multicolor flow cytometry analysis. Briefly, cells were obtained by lavage (BALC) or by homogenizing the MLN manually between frosted glass slides followed by filtration. $10^{6}$ cells were plated in a 96 -well round bottom plate. Cells were washed and blocked for non-specific staining using mouse anti-rat CD32 (FCyRII) antibody (BD Pharmingen ${ }^{\mathrm{TM}}$; San Diego, CA). Cell were then fluorescentlylabeled with one or a combination of the following mouse-anti-rat antibodies to determine phenotype: CD3 (T cells), CD3 and CD4 (T-helper cells), CD3 and CD8a (cytotoxic T cells), CD161a (NKR-P1A; natural killer cells), or CD45R (B220; B Cells) $\left(\mathrm{BD}\right.$ Pharmingen $\left.{ }^{\mathrm{TM}}\right)$. Cells were then washed and fixed with BD Cytofix/Cytoperm ${ }^{\mathrm{TM}}$ (BD Pharmingen ${ }^{\mathrm{TM}}$ ) and stored at $4{ }^{\circ} \mathrm{C}$ until phenotyped using a BD ${ }^{\mathrm{TM}}$ LSRII flow cytometer and BD FACSDiva ${ }^{\text {TM }}$ software v6 (Becton, Dickinson and Company; San Jose, CA) (within $24 \mathrm{~h}$ ). Results were gated and analyzed using @FlowJo Software v10 (FlowJo, LLC; Ashland, OR). 
Histopathology. The left lungs of rats were fixed with $10 \%$ neutral buffered formalin by airway pressure fixation under $30 \mathrm{~cm}$-water pressure to total lung capacity for $15 \mathrm{~min}$. Lung volumes were assessed by weight of water displacement. The left lungs were embedded in paraffin, sectioned onto slides, and stained with hematoxylin and eosin (H\&E) or trichrome. An $n=5$ stained slides per treatment group per time-point per study block were assessed; blocks were designed with paired controls resulting in $n=10$ for saline and SIL $233 \mu \mathrm{g}$ groups from each of the blocks when combined. Stained slides were analyzed for indications of inflammation, injury, and fibrosis by a certified veterinary pathologist at Charles River Laboratories (Wilmington, MA) who was blinded to the treatment groups. Indices of pathology were scored on scale of $0-5$, where $0=$ no observed effect, 1 = minimal response, 2 = mild response, $3=$ moderate response, 4 $=$ marked response, and $5=$ severe response.

Morphometric Analysis of Connective Tissue Thickness. Tissue sections from left lung were deparaffinized and stained with Sirius Red for detection of connective tissue, particularly fibrillar collagen. Slides were immersed in $0.1 \%$ picrosirius solution (100 mg of Sirius Red F3BA in $100 \mathrm{ml}$ of saturated aqueous picric acid) for $2 \mathrm{~h}$ followed by washing for $1 \mathrm{~min}$ in $0.01 \mathrm{~N} \mathrm{HCl}$. Slides were then counterstained with hematoxylin for 2 min, dehydrated, and mounted with a coverslip for imaging. Quantitative morphometric methods were used to measure the average thickness of Sirius Red-positive connective tissue in the alveolar regions. Volume ( $\%$ of the alveolar wall) and thickness were measured by standard morphometric analyses $[509,510]$. This consisted of basic point and intercept counting. An eyepiece counting overlay consisting of 11 by 11 lines (121 total points for each throw of the overlay) was used with a 40X objective. A grid pattern for throws of the counting overlay was used in order to ensure a uniform sampling of the section, which did not overweight interior points. The counting overlay throws of the eyepiece were positioned over the section at 12 uniformly spaced grid points in both $X$ and $Y$ coordinates. These 12 grid points were determined using the stage micrometer scale to measure the $X$ and $Y$ bounds of the section. Using the bounding rectangle of these coordinates a 3 by 4 grid was selected and the 12 intersections were used as the center point for each of the eyepiece counting overlay throws. Volume was determined by counting the number of points over the Sirius Red-positive connective tissues in the 
alveolar regions. Surface density of the alveolar wall was determined from intercepts between a line overlay and the alveolar wall. To limit the measurements to alveolar parenchyma, areas containing airways or blood vessels $25 \mu \mathrm{m}$ in diameter was excluded from the analysis. Average thickness of the Sirius Red-positive connective tissue of the alveolar wall was computed from two times the ratio of volume density of point to the surface density of the alveolar wall.

Bacterial Clearance from the Lung. To measure the clearance of bacteria from the lung, left lungs were excised and placed in $10 \mathrm{ml}$ of sterile deionized water. Lungs were homogenized using a Polytron®PT-2100 homogenizer with PT-DA 2112/EC aggregate attachment (Kinematica; Bohemia, NY). Homogenates were diluted and cultured on prepared Brain Heart Infusion Agar plates (Becton, Dickinson and Company). Cultures were incubated at $37^{\circ} \mathrm{C}$ for $24 \mathrm{~h}$. Cfu were counted and multiplied according to culture dilution factors.

Statistical Analyses. Results for all BAL parameters, cellular phenotype, morphometry and bacterial clearance were expressed as mean fold change from control \pm standard

error calculated as $\frac{\Delta \bar{x}}{\text { control } \bar{x}}$ and a one way analysis of variance (ANOVA) was performed between all treatments groups at each time point. Significant differences among groups were determined using the Student-Newman-Keuls post-hoc test. Data were analyzed using SigmaPlot for Windows Version 12.5 (Systat Software, Inc, Ekrath, Germany). For all analyses, significance was set at $p<0.05$. Because data from histopathology studies are inherently categorical, nonparametric analysis of variance was assessed using SAS/STAT software, Version 9.1, of the SAS System for Windows statistical programs (SAS Institute, Inc.; Cary, NC). In this instance groups were compared using the Wilcoxon rank sum test.

\section{Results}

Particle Characterization. One $\mathrm{ml}$ aliquots of $7.89 \mu \mathrm{g}$ dose DPM, $50 \mu \mathrm{g}$ dose DPM, $233 \mu \mathrm{g}$ dose of SIL, as well as $7.89 \mu \mathrm{g}$ and $50 \mu \mathrm{g}$ DPM in combination with SIL $233 \mu \mathrm{g}$ (DS) in PBS solution, were dried on carbon filters and examined using FESEM in order to characterize size of the primary particles and their degree of agglomeration. In 
solution, DPM was found to agglomerate to a size of approximately $2 \mu \mathrm{m}$ in diameter. The primary particle size was found to be smaller in size, $\sim 200 \mathrm{~nm}$. The primary particles of SIL were approximately $2 \mu \mathrm{m}$ in size and were evenly distributed in suspension (Figure 1A, 1B). The association between DPM and SIL in solution was minimal. EDX was used to evaluate the elemental components of each particle. DPM was found to be composed of primarily carbon, reflecting the carbonaceous core of the DPM, with very little detectable trace elements including: Chromium, Copper, Iron, Nickel, Oxygen, Silicon, and Zinc adsorbed to it (Figure 1C). The EDX spectrum of the SIL sample is depicted in Figure 1D.

Time-Course Study. Lung injury was evaluated by examining LDH in the BALF collected from rats at 1 day, 1 week, 1 month, 2 months, and 3 months post-exposure to particles. LDH activity was significantly increased in all groups that contained the high dose of SIL $(233 \mu \mathrm{g})$, and the high dose of SIL alone at all time points following exposure when compared to control (Figure 2). At early time points, the increase in groups that were co-exposed to SIL and DPM was primarily driven by SIL particles. However, by 3 months, co-exposure to the high doses of DPM and SIL (DS 50/233) resulted in an increase when compared to all other groups. There were no significant increases in LDH activity in groups exposed to DPM alone at either dose, SIL 50 alone, or DS $50 / 50$.

Protein mediators were evaluated in BALF as a measure of inflammation and immune response (Table 1). The low dose of DPM (DPM 7.89) and the low dose of silica (SIL 50) did not cause any significant changes in BALF protein when compared to control with the exception of DPM 7.89, whereby IL-10 was significantly elevated (data not shown). The high dose of SIL caused increases in several proteins associated with inflammation; MCP-1, MIP-1 $\alpha$, RANTES, GRO/KC, MIP-2, IP-10, TNF- $\alpha$, and IFN- $\gamma$ compared with the control and groups exposed to DPM only. SIL 233 alone and the DS 50/233 groups caused a decrease in the anti-inflammatory protein IL-10 from 1 week to 2 months post-exposure compared with control and DPM exposed groups. SIL 233 alone and the DS 50/233 groups also caused a significant increase in MMP-9, a protein associated with tissue remodeling that has implications in the development of fibrotic 
changes in the lung, at all time-points following exposure. Additionally, SIL high dose exposure increased MMP-2 and OPN, additional proteins associated with tissue remodeling, wound healing, and fibrosis at one month post-exposure, and at all time points thereafter for OPN. Finally, the SIL high dose alone or in combination with the DPM high dose caused an increase in IL-18, a protein associated with upregulation of the NLRP3/NALP3 inflammasome, at later time-points. DPM 50 alone did not illicit an increase in pro-inflammatory cytokines. However, the high dose of DPM did cause a decrease in IL-2 and IFN- $\gamma$ proteins associated with the effector properties of $\mathrm{T}_{\mathrm{h}} 1$ cells, when compared with controls. The decrease in IL-2 was also present in the high dose co-exposure group (DS 50/233). Similarly, DPM in combination with SIL exhibited independent action on the expression of some cytokines like, MIP- $\alpha$, GRO/KC, MIP-2, MMP-2 and MMP-9 at some time-points (Table 1), suggesting that DPM in a coexposure may have the capability to reduce the effects observed with SIL alone. These observations may indicate altered macrophage and lymphocyte activity in response to treatment with DPM. There were no significant changes in the following cytokines in any

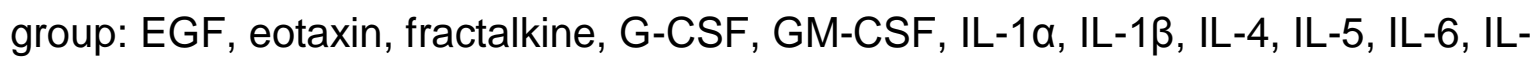
12p70, IL-13, IL-17A, leptin, LIX, TGF- $\beta$, TIMP-1, and VEGF (data not shown).

Cell differentials were performed on BALC to further assess inflammation (Figure $3 A \& B$ ). DPM alone at either dose did not cause an increase in the influx of AMs (Figure $3 \mathrm{~A}$ ) or neutrophils (Figure 3B). AM influx was increased in all groups that contained SIL 233 beginning at 1 week post-exposure. Neutrophil influx significantly increased as early as 1 day post-exposure in all groups containing SIL 233 (Figure 3B), and persisted throughout the time course. Exposure to SIL $50 \mu \mathrm{g}$ showed a trend toward an increase in neutrophils up to 1 month following exposure with resolution of response over time. At 1 month, exposure to DS 50/233 caused a significant increase in neutrophils when compared to all groups. The DS50/50 group followed a similar pattern as SIL 50 alone. BALC activation was also evaluated as oxidant production ex vivo (Figure $3 \mathrm{C} \& D$ ). DPM only doses used in this study did not cause an increase in oxidant production by AMs and neutrophils as measured by CL. Oxidant production in the SIL 233 group significantly increased for both total phagocytes at 1 day and at 3 months post-exposure (Figure $3 \mathrm{C}$ ), and oxidant production by macrophages followed a similar pattern 
(Figure 3D). Interestingly, exposure to the higher dose of DPM combined with the high dose of SIL resulted in significantly increased oxidant production over exposure to the high dose of SIL alone at 1 week, 1 month, and 2 months post-exposure, suggesting a higher oxidant burden in the lung of rats in the high dose co-exposure group at these times.

BAL and MLN lymphocytes were phenotyped by flow cytometry. In addition to phagocytic influx described in Figure 3, exposure to SIL at the high dose alone or in combinations with DPM 50 caused significantly increased lymphocyte influx into the lung as early as 1 day following exposure (Table 2) and proliferation in the MLN as early as 1 week post-exposure (Table 3). This effect was attributed primarily to CD4 ${ }^{+}$and $\mathrm{CD}^{+} \mathrm{T}$ cell population subsets. Exposure to DPM 50 alone did not cause a significant increase in lymphocyte populations in BAL or MLN. Additionally, it was noted that for the co-exposed group, DPM exhibited independent action reducing the influx of the CD8 ${ }^{+}$subset of cells into the lung observed with the SIL only exposed group at 1 month and total $T$ cells, which was mostly attributed to reduced influx of the CD4 ${ }^{+} \mathrm{T}$ cell subset, at 3 months following exposure.

H\&E and trichrome-stained tissue sections were evaluated by a board certified veterinarian pathologist (Table 4). DPM alone at either dose did not cause pathological changes in the lung. Influx of AMs, as a measure of inflammation, was significantly elevated in the SIL 233 group alone at all time-points following exposure and when combined with DPM at 1 month following exposure. Alveolar epithelial hyperplasia (AEH) was also observed at all points following exposure in DS 50/233 exposed groups. At 1 month following exposure to particles, minimal to mild septal fibrosis was observed in groups exposed to SIL 233. Interestingly, incidence of septal fibrosis was significantly increased by co-exposure to the high doses of DPM and SIL at 1 month. By 3 months after exposure, occurrence of septal fibrosis was equivalent in both SIL 233 only and co-exposed groups. Groups exposed to SIL in combination with high dose DPM scored significantly higher for particles in tissue versus DPM alone as indicated by the presence of brown granules accumulated in macrophages, which corresponds to an observation of increased coloration in lymph nodes as well. Furthermore, mild 
hyperplasia of the bronchiolar/alveolar epithelium was observed near the terminal bronchioles at 2 months following exposure and was found to be associated with the presence of macrophages loaded with high particle content, suggesting a correlation between tissue damage and the presence of particles (data not shown). SIL 50 and DS $50 / 50$ were not evaluated. Overall, most histopathological changes were attributable to exposure to $233 \mu \mathrm{g}$ of SIL; however, the presence of DPM often increased the severity and/or incidence of these changes in the co-exposure groups.

To further quantify observations of fibrosis, alveolar expansion, alveolar septal wall thickness, and thickness of fibrillar collagen were measured by morphometry to further assess the fibrotic response to exposure. Alveolar expansion was measured as a ratio of the total airspace volume to volume versus the surface to volume of the epithelial surface intercepts or thickness of airspace between alveolar tissues. Alveolar septal wall thickness was measured as a ratio of the total volume to volume of alveolar tissue versus the surface to volume of epithelial surface intercepts. No significant differences were observed between the treatment groups. Additionally, fibrillar collagen thickness was measured as the ratio of total collagen volume to volume versus total epithelial surface intercepts surface to volume. This measurement is indicative of fibrotic development in lung tissue. Although not statistically significant, there was a trend for increased fibrillar collagen volume in SIL 233-exposed groups and this trend was further increased for co-exposed groups at the latest time points measured (data not shown). Infection Study. In order to investigate the effects of particle exposure on respiratory susceptibility to a bacterial pathogen, an infectivity study was conducted to assess the clearance of $L$. monocytogenes from the lung over a 14 day time course 1 week following exposure to particles. Despite lung inflammation and phagocytic cell activity observed in the absence of infection in co-exposed groups (Figure 3), overall clearance of the pathogen was not significantly altered between these treatment groups (Figure $4 A$ ). The data suggest that, for the doses of DPM used in this study, which were not found to induce toxicity by themselves, combination with moderately toxic doses of SIL did not alter susceptibility to an infection with Listeria monocytogenes. Influx of cells into the lungs was significantly increased for SIL 233 exposed groups at day 1 post-infection 
(Figure 4). This influx appeared to be comprised mostly of neutrophils (Figure 4D) and lymphocytes. By day 3 post-infection, the influx of cells in SIL 233-exposed groups was resolved. Finally, cells from the BAL and MLN were phenotyped as in the time-course study to evaluate lymphocyte populations. For the SIL 233-exposed group the influx of lymphocytes into the BAL at day 1 post-infection was significantly greater when compared with the control group, and again on day 14. DPM exposure caused no significant alterations in lymphocyte influx into the lung, while combined exposure to particles caused significant increases in total lymphocyte influx at day 14 compared with control and DPM exposed groups comprised mostly of CD4+ cells (Table 5). In the MLN, the SIL 233-exposed group had significantly increased lymphocytes, specifically $\mathrm{CD}^{+}{ }^{+} \mathrm{T}$ Cells, compared with control and overall $\mathrm{T}$ cell populations were significantly increased when compared to control and DPM 50 at day 1 post-infection. SIL-233 exposure also increased total lymphocytes in the MLN compared with all other groups at day 3 post infection. DPM exposure caused significant decreases compared with SILexposed group for total $\mathrm{T}$ Cells at day 3 post-infection and caused significant decreases in the CD8 ${ }^{+}$subset of T cells compared with SIL-233 and control groups for the same time-point. Finally, combined exposure caused increased lymphocytes at Day 1 for total cells and total T cells compared with control group and compared with DPM and control for the $\mathrm{CD} 4^{+} \mathrm{T}$ cell subset (Table 6 ).

\section{Discussion}

The current studies were conducted in order to address a knowledge gap with relation to co-exposures representative of those that may occur in mining industry occupations, including hydraulic fracturing. It is important to note that the DPM used in this study was extracted from DE collected from an industrial forklift in the 1990s, which qualifies it as "transitional" in nature (collected between 1988 and 2006). Transitional DE samples vary from "traditional" DE or "TDE" (collected prior to 1988) and New Technology DE or "NTDE" (collected post-2006) in chemical and physical properties. TDE is considered to be more hazardous in nature due to having a larger amount of particulate matter, carbon monoxide, and hydrocarbon emissions, while NTDE has significantly lower TDE-related emissions, [511]; however, very little information is 
available on health outcomes related to NTDE exposure. Additionally, the current study examined DPM only and not total DE, which contains higher levels of metals and hydrocarbons, which in turn may lead to greater toxicity following exposure to DE. The silica used in this study was also a surrogate for occupational exposures that occur in mining operations and oil and gas extraction. It is not a freshly fractured form of crystalline silica, which has been shown to be higher in toxicity than aged silica [487], and although sand used at hydraulic fracturing sites contains respirable silica, composition of the sand may vary from site to site [512, 513].

While most parameters of toxicity evaluated could be attributed to the more reactive and toxic crystalline silica particles, DPM, at doses that represent different durations of exposure based on field measures taken at mining sites and OELs, did exacerbate the effects of silica in the acute exposure model examined, particularly between 1 week and 1 month post-exposure. This effect was observed for the parameters of pulmonary inflammation, quantified by the influx of neutrophils into the lung (Figure 3B) as well as by the oxidant production by phagocytes (Figure $3 \mathrm{C}, \mathrm{D}$ ) at time points up to one month for inflammation and up to three months for oxidant production. Furthermore, assessment of pathology in the lung tissue suggested that DPM may be contributing to the mild fibrotic response induced by silica in the lung, whereby there was an increased incidence of mild fibrosis at 1 month post-exposure in the animals that were exposed to the combination of the high doses of particulate. Additionally, while silica contributed to an increase in lymphocyte- and immune-related cytokines in the lungs (Tables 1-3), DPM reduced the expression of many proteins including: MIP-1 $\alpha$, GRO/KC, MIP-2, IL-2, IFN-y, MMP-2, and MMP-9, in the BALF when in combination with silica, especially at early time-points. DPM may exhibit independent action on macrophage activity, as well as lymphocyte influx and proliferation thus attenuating the effects induced on these cell types by silica alone (Table 1-3). Immunological alterations were further examined by evaluating susceptibility to an infection following particulate exposure. While some alterations in immune cellular influx were observed in silica-exposed groups (Tables 6-7), the exposures did not alter the bacterial clearance rate or resolution of infection. 
The toxicological properties of crystalline silica are well-established. Silica is known to cause fibrotic changes in the lung following both acute exposures to bolus doses of particles and chronic lower dose exposure to particles [514]. The mechanism directing the toxicity of silica particles in the lung still remains ambiguous but is thought to stem from multiple attributes relating to the crystalline structure of silica and its reactivity based on either surface charge or silicon-containing groups present on the surface of silica due to disruptions made to its structure during crushing, grinding, and drilling [514]. In conjunction with this, reactive oxygen/nitrogen species (ROS/RNS) associated with the particles themselves or as products of the respiratory burst following activation of phagocytes have been shown to drive a prolonged state of inflammation in the lungs $[487,490,515,516]$. Studies investigating pulmonary toxicity of diesel demonstrate DPM-induced inflammation and oxidative stress as well [467, 473-476]; however, unlike silica, DPM exposure does not have the same propensity to cause fibrosis. Instead, chronic exposures to DE/DPM have been shown to cause disease outcomes including chronic obstructive pulmonary disorder, emphysema, and cancer $[411,415,517,518]$.

In regards to potential mechanisms in the development of fibrosis, crystalline silica particles have been shown to either bind to scavenger receptors on alternatively activated macrophages (M2) followed by uptake into the cell in a phagosome, or the particles are phagocytosed by a classically activated macrophage (M1) in the interstitium once the M2 macrophages become overloaded [128]. M2s are phenotypically distinct from M1s, being activated primarily by IL-4 and IL-13 rather than IFN-y and are typically less involved in killing of microbes/clearance and more associated with functions of wound healing and collagen development [519]. As reviewed by Kawasaki et al. and demonstrated in original work by Joshi et al. [128, 130, 520], the uptake of these particles triggers a signaling cascade within macrophages that leads to: the release of recruitment factors for additional inflammatory/immune cells, stimulation of the autocrine and paracrine production of fibrogenic mediators, and, most notably, either the successful clearance of particles from the lung to either the GI tract or lymphatics or the eventual phagolysosomal destruction and death of the cell that engulfed the particles. Directly following the death of a macrophage containing silica, 
the particles are engulfed by another nearby phagocyte. This repeated cycle of uptake, release, and re-uptake continues in the lung until a point when cellular signals lead to the formation of a granuloma to sequester particles in an effort to reduce damage to the surrounding tissue [521-523]. It is hypothesized that M1 type macrophages may initiate this process [128], but other cell types including lymphocytes, epitheliod monocytes and macrophages, and giant cells are involved as well [91, 524, 525].

In relationship to the fibrotic paradigm discussed above, this study showed that there were significant increases in neutrophil and lymphocyte recruitment in the high dose co-exposed group when compared to the silica-exposed group, along with significant increases in cytotoxicity in the lung measured as LDH in BALF, and this was accompanied by the increase in incidence of mild fibrosis at 1 month post-exposure in the high dose co-exposure group. While the interaction of silica with receptors was not investigated in the present study, downstream mediators of these interactions were increased in the BALF. Increases in chemotactic/recruitment proteins associated with inflammation (MCP-1 (CCL2), MIP-1a (CCL3), RANTES (CCL5), GRO/KC (CXCL1), MIP-2 (CXCL2), IP-10 (CXCL10), and TNF- $\alpha$ ) were significantly increased in the groups containing the high dose of silica, and the increases tended to progress throughout the time-course (Table 1); whereas, the anti-inflammatory protein, IL-10, was significantly decreased in silica-exposed groups throughout the time-course. Additionally, OPN, MMP-2, and MMP-9, proteins involved in tissue remodeling, were also significantly increased in silica-exposed groups, particularly later in the time-course. Though differences between the high dose co-exposed group and high dose silica-exposed group separately were not statistically significant for this parameter, there was approximately a $30 \%$ increase in MMP-9 expression, at the latest time-point, suggesting a trend for increased tissue remodeling in the co-exposed group over that of silica alone.

In addition to the pro-inflammatory responses discussed above, a significant increase in IL-18 at later time-points following silica exposure was measured for both the high dose co-exposed and high dose silica only groups. The NLRP3 inflammasome is an immune system mediator that detects danger signals produced by damaged cells. 
The inflammasome is also activated by stimulation with foreign bioreactive particles like multi-walled carbon nanotubes, which cause production of oxidants by phagocytic cells leading to up-regulation of NLRP3 complex formation, resulting in the cleavage of IL-18 and IL-1 $\beta$ and prolonged inflammation and fibrotic development in the lung [526-530]. Crystalline silica exposure has also been shown to upregulate the activity of the NALP3/NLRP3 inflammasome following binding to scavenger receptors on macrophages both in vitro $[123,531,532]$ and in vivo [532] leading to the cleavage of IL-1 $\beta$ and IL-18 to their active forms by caspases leading to apoptosis or pyroptosis in cells $[123,131]$. While we did not observe an increase in IL-1 $\beta$ we did observe an increase in IL-18 for both the SIL 233 and high dose co-exposed groups. At time-points as early as 1 week following exposure, IL-18 was significantly increased in both groups compared with DPM 50, but by 3 months both groups were significantly greater than both DPM 50 and control (Table 1). Therefore, inflammasome activation may also be occurring as an early response in the development of fibrosis.

In addition to the pro-inflammatory properties of both DPM and crystalline silica, both particles have been shown to alter mechanisms of pulmonary immunity, although the effects following exposure to these particles differ in this regard. Silica has been shown to cause hyper-activation and hyper-responsiveness of both macrophages [92, 402] and T-cells [52, 91]. This was also demonstrated in the current study in the group exposed only to the high dose of silica individually. Conversely, DPM has been shown to suppress activation and effector functions of macrophages and T cells, and this suppression has been associated with increased susceptibility to infection and reduced pulmonary clearance of pathogens [316, 324, 402, 483, 485, 533-535]. Because both DPM and silica exposure have been shown to increase lung inflammation and phagocytic recruitment in acute exposures, but lead to decreased clearance following chronic exposure, it was of interest to determine whether the capacity for clearance of a pathogen in an acute model of exposure to the particles in combination would be altered. In the present study, a difference in bacterial clearance from the lungs was not observed following exposure to DPM, silica, or the DPM and silica co-exposure (Figure $4 \mathrm{~A}$ ); this may reflect the lower doses delivered here when compared to previous studies $[59,62,316,324,481,497]$. Other differences among studies include the degree to 
which DPM or SIL were freshly collected, thus making them less reactive than particles that workers in the field may be exposed to [514, 536, 537], and the engine source of the DPM, which would determine the elemental constituents. In addition, the timing of the infection may influence clearance of the infection following exposure. In studies that examined a pathogenic response more acutely (within 3 days of particle exposure), DPM exposure reduced clearance of the infection [316, 324, 481]. In response to silica exposure, clearance of bacteria has been shown to be critically dependent upon whether exposure is acute [538], resulting in increased clearance of infection due to enhanced inflammatory and essentially a "primed" macrophage response for effective uptake and clearance of the bacteria, or chronic, resulting in decreased clearance [59, $62,497]$ suggesting the degree of lung injury present would alter susceptibility to infection. Further investigation is needed to determine the effects that particles might have on clearance and susceptibility to infections experienced more subsequently to particle exposure. Given that we observed development of fibrosis at later time-points, administering bacteria later in the time-course, such as at 1,2, or 3 months may result in more similar effects to what has been observed in chronic silica exposures previously $[59,62,497]$.

\section{Conclusion}

The data taken together show that DPM exposure at doses that do not elicit pulmonary toxicity has the capacity to significantly alter silica-induced pulmonary effects, primarily inflammation, oxidant production, and rate of onset of fibrotic changes in the lung, as well as exhibit independent action, which mediates some immune cell activity when the exposure occurs simultaneously as a mixture. The current study does not delineate the specific interactions between the two particulates in the lung following exposure and leading to the observed effects. One possibility is that presence of and/or injury induced by crystalline silica alters the clearance rate of the DPM particles from the lung, which in turn results in a longer persistence of DPM in the lung in conjunction with silica. DPM alone may be cleared at a greater rate resulting in the lack of observed toxicity following the individual exposures in this study. This hypothesis is supported by the evidence from the histopathology analysis in the observance of brown granules 
(DPM) in the lungs of only the co-exposed group and by the presence of DPM particles in the lymph nodes of only the co-exposed group at the later time points and not the DPM-only exposure. Another possibility for the observed effects may be related to the particle load as a result of DPM and crystalline silica delivered to the lung simultaneously. Although the delivered doses by intratracheal instillation in these studies are lower than many previous studies [324, 481, 497, 538], the co-exposed group received a total particle load of $283 \mu \mathrm{g}$ compared to the individual silica load of $233 \mu \mathrm{g}$ and the DPM load of $50 \mu \mathrm{g}$, which is an increase in load of $\sim 18 \%$. Studies are underway to address the effects of load and clearance in relationship to the combined exposure of DPM and silica. 
Table 1: Immunoregulatory Proteins Recovered from BALF of Sprague-Dawley Rats Exposed to DPM, SIL, or Combination of DPM and SIL

\begin{tabular}{|c|c|c|c|c|c|c|c|c|c|c|c|c|c|c|c|}
\hline \multirow{4}{*}{ 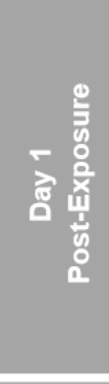 } & $\begin{array}{c}\text { Group \& } \\
\text { Dose } \\
(\mu g)\end{array}$ & MCP-1 & MIP-1a & RANTES & GRO/KC & MIP-2 & IP-10 & TNF- $\alpha$ & IFN-V & IL-2 & IL-10 & OPN & MMP-2 & MMP-9 & IL-18 \\
\hline & DPM 50 & $\begin{array}{c}0.95 \\
\pm 0.36\end{array}$ & $\begin{array}{c}1.02 \\
\pm 0.93\end{array}$ & $\begin{array}{l}-0.21 \\
\pm 0.12\end{array}$ & $\begin{array}{c}1.27 \\
\pm 1.34\end{array}$ & $\begin{array}{c}0.70 \\
\pm 0.45\end{array}$ & $\begin{array}{c}3.05 \\
\pm 1.03\end{array}$ & $\begin{array}{c}0.19 \\
\pm 0.21\end{array}$ & $\begin{array}{l}-0.96 \\
\pm 0.04\end{array}$ & BLD & $\begin{array}{c}0.78 \\
\pm 0.41\end{array}$ & $\begin{array}{l}-0.19 \\
\pm 0.12\end{array}$ & $\begin{array}{c}0.63 \\
\pm 0.14\end{array}$ & $\begin{array}{c}0.60 \\
\pm 0.89\end{array}$ & $\begin{array}{c}0.14 \\
\pm 0.05\end{array}$ \\
\hline & SIL 233 & $\begin{array}{c}2.11 \\
\pm 0.69\end{array}$ & $\begin{array}{c}2.37 \\
\pm 0.73\end{array}$ & $\begin{array}{c}0.13 \\
\pm 0.23\end{array}$ & $\begin{array}{c}4.59 \\
\pm 0.65^{+}\end{array}$ & $\begin{array}{c}1.16 \\
\pm 0.15\end{array}$ & $\begin{array}{c}4.01 \\
\pm 0.97 @\end{array}$ & $\begin{array}{c}1.64 \\
\pm 0.47\end{array}$ & $\begin{array}{c}1.41 \\
\pm 0.94^{\wedge}\end{array}$ & $\begin{array}{c}2.18 \\
\pm 2.97\end{array}$ & $\begin{array}{c}0.34 \\
\pm 0.30\end{array}$ & $\begin{array}{c}0.55 \\
\pm 0.28\end{array}$ & $\begin{array}{c}1.56 \\
\pm 0.38^{*}\end{array}$ & $\begin{array}{c}3.72 \\
\pm 0.53^{*}\end{array}$ & $\begin{array}{c}0.41 \\
\pm 0.15\end{array}$ \\
\hline & $\begin{array}{c}\text { DS } \\
50 / 233\end{array}$ & $\begin{array}{c}1.29 \\
\pm 0.65\end{array}$ & $\begin{array}{c}0.98 \\
\pm 0.43\end{array}$ & $\begin{array}{l}-0.08 \\
\pm 0.15\end{array}$ & $\begin{array}{c}0.81 \\
\pm 0.89\end{array}$ & $\begin{array}{c}0.52 \\
\pm 0.29\end{array}$ & $\begin{array}{c}3.09 \\
\pm 1.32\end{array}$ & $\begin{array}{c}1.56 \\
\pm 0.57^{\star}\end{array}$ & $\begin{array}{l}-0.85 \\
\pm 0.07\end{array}$ & BLD & $\begin{array}{c}0.04 \\
\pm 0.30\end{array}$ & $\begin{array}{c}0.89 \\
\pm 0.35^{\star}\end{array}$ & $\begin{array}{c}0.89 \\
\pm 0.19\end{array}$ & $\begin{array}{c}2.86 \\
\pm 0.52^{*}\end{array}$ & $\begin{array}{c}0.24 \\
\pm 0.12\end{array}$ \\
\hline \multirow{3}{*}{ 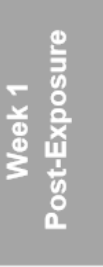 } & DPM 50 & $\begin{array}{l}-0.71 \\
\pm 0.16\end{array}$ & $\begin{array}{l}-0.27 \\
\pm 0.05\end{array}$ & $\begin{array}{c}0.07 \\
\pm 0.22\end{array}$ & $\begin{array}{l}-0.32 \\
\pm 0.07\end{array}$ & $\begin{array}{c}0.24 \\
\pm 0.19\end{array}$ & $\begin{array}{c}1.02 \\
\pm 0.79\end{array}$ & $\begin{array}{l}-0.26 \\
\pm 0.11\end{array}$ & BLD & BLD & $\begin{array}{l}-0.10 \\
\pm 0.34\end{array}$ & $\begin{array}{l}-0.14 \\
\pm 0.08\end{array}$ & $\begin{array}{c}0.10 \\
\pm 0.19\end{array}$ & $\begin{array}{c}1.38 \\
\pm 0.80\end{array}$ & $\begin{array}{l}-0.22 \\
\pm 0.11\end{array}$ \\
\hline & SIL 233 & $\begin{array}{c}1.31 \\
\pm 0.50\end{array}$ & $\begin{array}{c}3.54 \\
\pm 0.95^{+}\end{array}$ & $\begin{array}{c}1.12 \\
\pm 0.22\end{array}$ & $\begin{array}{c}3.76 \\
\pm 0.24^{+}\end{array}$ & $\begin{array}{c}0.68 \\
\pm 0.19\end{array}$ & $\begin{array}{c}4.05 \\
\pm 1.20\end{array}$ & $\begin{array}{c}0.42 \\
\pm 0.19^{*}\end{array}$ & $\begin{array}{l}-0.50 \\
\pm 0.22\end{array}$ & $\begin{array}{c}1.43 \\
\pm 1.56\end{array}$ & $\begin{array}{c}-0.95 \\
\pm 0.03^{*}\end{array}$ & $\begin{array}{c}0.01 \\
\pm 0.06\end{array}$ & $\begin{array}{l}-0.05 \\
\pm 0.12\end{array}$ & $\begin{array}{c}29.25 \\
\pm 5.36^{+}\end{array}$ & $\begin{array}{c}0.32 \\
\pm 0.08 \&\end{array}$ \\
\hline & $\begin{array}{c}\text { DS } \\
50 / 233\end{array}$ & $\begin{array}{c}1.35 \\
\pm 1.11\end{array}$ & $\begin{array}{c}1.13 \\
\pm 0.19\end{array}$ & $\begin{array}{c}0.57 \\
\pm 0.48\end{array}$ & $\begin{array}{c}1.32 \\
\pm 0.61^{*}\end{array}$ & $\begin{array}{c}0.31 \\
\pm 0.08\end{array}$ & $\begin{array}{c}3.15 \\
\pm 1.42\end{array}$ & $\begin{array}{c}0.34 \\
\pm 0.06^{*}\end{array}$ & BLD & BLD & $\begin{array}{c}-0.83 \\
\pm 0.09^{\star}\end{array}$ & $\begin{array}{c}0.19 \\
\pm 0.20\end{array}$ & $\begin{array}{l}-0.08 \\
\pm 0.12\end{array}$ & $\begin{array}{c}14.55 \\
\pm 2.05^{\star}\end{array}$ & $\begin{array}{c}0.29 \\
\pm 0.10^{*}\end{array}$ \\
\hline \multirow{3}{*}{ 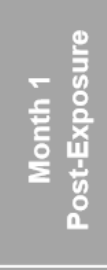 } & DPM 50 & $\begin{array}{l}-0.38 \\
\pm 0.28\end{array}$ & $\begin{array}{l}-0.04 \\
\pm 0.06\end{array}$ & $\begin{array}{c}0.11 \\
\pm 0.12\end{array}$ & $\begin{array}{l}-0.43 \\
\pm 0.08\end{array}$ & $\begin{array}{l}-0.02 \\
\pm 0.08\end{array}$ & $\begin{array}{l}-0.59 \\
\pm 0.09\end{array}$ & $\begin{array}{l}-0.07 \\
\pm 0.11\end{array}$ & BLD@ & BLD & $\begin{array}{l}-0.44 \\
\pm 0.15\end{array}$ & $\begin{array}{l}-0.11 \\
\pm 0.06\end{array}$ & $\begin{array}{l}-0.09 \\
\pm 0.11\end{array}$ & $\begin{array}{c}0.09 \\
\pm 0.43\end{array}$ & $\begin{array}{l}-0.62 \\
\pm 0.02\end{array}$ \\
\hline & SIL 233 & $\begin{array}{c}26.06 \\
\pm 7.70^{*}\end{array}$ & $\begin{array}{c}4.73 \\
\pm 0.98^{*}\end{array}$ & $\begin{array}{c}1.33 \\
\pm 0.15^{\star}\end{array}$ & $\begin{array}{c}2.32 \\
\pm 0.22^{*}\end{array}$ & $\begin{array}{c}0.41 \\
\pm 0.10^{*}\end{array}$ & $\begin{array}{c}1.88 \\
\pm 0.87\end{array}$ & $\begin{array}{c}0.48 \\
\pm 0.35\end{array}$ & $\begin{array}{l}-0.37 \\
\pm 0.27\end{array}$ & $\begin{array}{c}1.65 \\
\pm 1.77\end{array}$ & $\begin{array}{l}-0.49 \\
\pm 0.16\end{array}$ & $\begin{array}{c}0.63 \\
\pm 0.21^{*}\end{array}$ & $\begin{array}{c}0.50 \\
\pm 0.15^{\star}\end{array}$ & $\begin{array}{c}17.67 \\
\pm 5.72^{*}\end{array}$ & $\begin{array}{l}-0.20 \\
\pm 0.09\end{array}$ \\
\hline & $\begin{array}{c}\text { DS } \\
50 / 233\end{array}$ & $\begin{array}{c}43.41 \\
\pm 9.70^{*}\end{array}$ & $\begin{array}{c}3.94 \\
\pm 1.06^{\star}\end{array}$ & $\begin{array}{c}1.19 \\
\pm 0.26^{*}\end{array}$ & $\begin{array}{c}1.96 \\
\pm 0.15^{\star}\end{array}$ & $\begin{array}{c}0.16 \\
\pm 0.10\end{array}$ & $\begin{array}{c}3.31 \\
\pm 1.80\end{array}$ & $\begin{array}{c}0.50 \\
\pm 0.31\end{array}$ & $\begin{array}{c}-0.96 \\
\pm 0.04 @\end{array}$ & BLD & $\begin{array}{c}-0.82 \\
\pm 0.10 @\end{array}$ & $\begin{array}{c}1.03 \\
\pm 0.25^{\star}\end{array}$ & $\begin{array}{c}0.61 \\
\pm 0.15^{\star}\end{array}$ & $\begin{array}{c}24.20 \\
\pm 4.85^{\star}\end{array}$ & $\begin{array}{l}-0.35 \\
\pm 0.09\end{array}$ \\
\hline \multirow{3}{*}{ 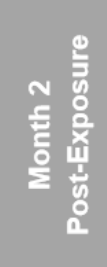 } & DPM 50 & $\begin{array}{c}0.17 \\
\pm 0.59\end{array}$ & $\begin{array}{c}0.09 \\
\pm 0.25\end{array}$ & $\begin{array}{c}0.33 \\
\pm 0.31\end{array}$ & $\begin{array}{l}-0.66 \\
\pm 0.12\end{array}$ & $\begin{array}{c}0.09 \\
\pm 0.08\end{array}$ & $\begin{array}{c}0.06 \\
\pm 0.21\end{array}$ & $\begin{array}{l}-0.28 \\
\pm 0.17\end{array}$ & $\begin{array}{c}0.36 \\
\pm 0.52\end{array}$ & $\begin{array}{c}0.29 \\
\pm 0.51\end{array}$ & $\begin{array}{c}0.09 \\
\pm 0.08\end{array}$ & $\begin{array}{l}-0.21 \\
\pm 0.17\end{array}$ & $\begin{array}{c}0.71 \\
\pm 0.34\end{array}$ & $\begin{array}{c}0.87 \\
\pm 0.76\end{array}$ & $\begin{array}{c}0.17 \\
\pm 0.24\end{array}$ \\
\hline & SIL 233 & $\begin{array}{c}23.26 \\
\pm 3.92^{*}\end{array}$ & $\begin{array}{c}5.42 \\
\pm 0.95^{\star}\end{array}$ & $\begin{array}{c}0.03 \\
\pm 0.18\end{array}$ & $\begin{array}{l}-0.28 \\
\pm 0.19\end{array}$ & $\begin{array}{c}0.10 \\
\pm 0.03\end{array}$ & $\begin{array}{c}1.85 \\
\pm 0.49^{*}\end{array}$ & $\begin{array}{c}0.36 \\
\pm 0.10^{\&}\end{array}$ & $\begin{array}{c}1.01 \\
\pm 0.64\end{array}$ & $\begin{array}{l}-0.11 \\
\pm 0.38\end{array}$ & $\begin{array}{c}-0.36 \\
\pm 0.03^{*}\end{array}$ & $\begin{array}{c}8.09 \\
\pm 1.78^{+}\end{array}$ & $\begin{array}{c}0.39 \\
\pm 0.18\end{array}$ & $\begin{array}{c}5.98 \\
\pm 1.74^{\star}\end{array}$ & $\begin{array}{c}1.43 \\
\pm 0.28^{+}\end{array}$ \\
\hline & $\begin{array}{c}\text { DS } \\
50 / 233\end{array}$ & $\begin{array}{c}19.90 \\
\pm 5.76^{*}\end{array}$ & $\begin{array}{c}4.93 \\
\pm 0.93^{*}\end{array}$ & $\begin{array}{l}-0.19 \\
\pm 0.13\end{array}$ & $\begin{array}{l}-0.27 \\
\pm 0.23\end{array}$ & $\begin{array}{l}-0.09 \\
\pm 0.07\end{array}$ & $\begin{array}{c}2.21 \\
\pm 0.75^{\star}\end{array}$ & $\begin{array}{c}0.34 \\
\pm 0.12^{\&}\end{array}$ & $\begin{array}{c}0.44 \\
\pm 0.47\end{array}$ & $\begin{array}{c}0.90 \\
\pm 0.83\end{array}$ & $\begin{array}{c}-0.41 \\
\pm 0.04^{*}\end{array}$ & $\begin{array}{c}3.49 \\
\pm 1.34 @\end{array}$ & $\begin{array}{c}0.12 \\
\pm 0.10\end{array}$ & $\begin{array}{c}4.37 \\
\pm 0.55 @\end{array}$ & $\begin{array}{c}0.81 \\
\pm 0.22 @\end{array}$ \\
\hline \multirow{2}{*}{ 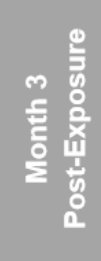 } & DPM 50 & $\begin{array}{l}-0.20 \\
\pm 0.36\end{array}$ & $\begin{array}{c}0.31 \\
\pm 0.17\end{array}$ & $\begin{array}{r}0.15 \\
\pm 0.16\end{array}$ & $\begin{array}{l}-0.21 \\
\pm 0.19\end{array}$ & $\begin{array}{c}0.05 \\
\pm 0.09\end{array}$ & $\begin{array}{l}-0.12 \\
\pm 0.21\end{array}$ & $\begin{array}{c}0.28 \\
\pm 0.38\end{array}$ & BLD & BLD & $\begin{array}{l}-0.20 \\
\pm 0.08\end{array}$ & $\begin{array}{l}-0.27 \\
\pm 0.07\end{array}$ & $\begin{array}{l}-0.12 \\
\pm 0.11\end{array}$ & $\begin{array}{c}0.52 \\
\pm 0.38\end{array}$ & $\begin{array}{c}0.10 \\
\pm 0.11\end{array}$ \\
\hline & SIL 233 & $\begin{array}{c}20.37 \\
\pm 5.62^{*}\end{array}$ & $\begin{array}{c}7.62 \\
\pm 1.59^{*}\end{array}$ & $\begin{array}{c}0.90 \\
\pm 0.11^{*}\end{array}$ & $\begin{array}{c}2.66 \\
\pm 0.35^{\star}\end{array}$ & $\begin{array}{c}0.25 \\
\pm 0.17\end{array}$ & $\begin{array}{c}4.83 \\
\pm 1.28^{*}\end{array}$ & $\begin{array}{c}1.05 \\
\pm 0.15 @\end{array}$ & $\begin{array}{c}1.12 \\
\pm 0.89\end{array}$ & $\begin{array}{l}-0.56 \\
\pm 0.22\end{array}$ & $\begin{array}{l}-0.40 \\
\pm 0.10\end{array}$ & $\begin{array}{c}1.08 \\
\pm 0.27^{*}\end{array}$ & $\begin{array}{l}-0.44 \\
\pm 0.09\end{array}$ & $\begin{array}{c}62.17 \\
\pm 23.11^{*}\end{array}$ & $\begin{array}{c}1.31 \\
\pm 0.35^{\star}\end{array}$ \\
\hline
\end{tabular}


All data are shown as mean fold change over control \pm standard error. ${ }^{*}$ Different from control and DPM $50 \mu g$; ${ }^{\wedge}$ different from DPM $50 \mu \mathrm{g}$ and DS 50/233 $\mu \mathrm{g}$ groups; +different from all other groups; ${ }^{\circledR}$ different from DPM $50 \mu \mathrm{g}$; ${ }^{\circledR}$ different from control. Results that were below the limit of detection (BLD) were noted; 1/2 of the lowest limit of detection was used for statistical analysis of values BLD. Statistical significance is $p \leq 0.05$. 
Table 2: Lymphocyte Phenotype Determination by Flow Cytometry in the BALF Recovered from Sprague-Dawley Rats Exposed to DPM, SIL, or DPM and SIL Combined

\begin{tabular}{|c|c|c|c|c|c|c|}
\hline \multirow{4}{*}{ 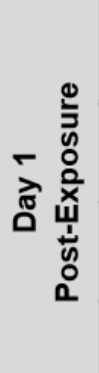 } & $\begin{array}{l}\text { Exposure } \\
(\mu \mathrm{g})\end{array}$ & $\begin{array}{c}\text { Total } \\
\text { Lymphocytes }\end{array}$ & T Cells & $\mathrm{CD}^{+} \mathrm{T}$ Cells & $\mathrm{CD}^{+} \mathrm{T}$ Cells & B Cells \\
\hline & DPM 50 & $0.34 \pm 0.20$ & $0.27 \pm 0.36$ & $0.97 \pm 0.77$ & $0.42 \pm 0.18$ & $0.11 \pm 0.24$ \\
\hline & SIL 233 & $1.81 \pm 0.44^{*}$ & $0.90 \pm 0.41$ & $1.41 \pm 0.74$ & $1.71 \pm 0.94$ & $0.66 \pm 0.35$ \\
\hline & DS 50/233 & $1.49 \pm 0.48^{*}$ & $0.68 \pm 0.39$ & $2.02 \pm 0.76$ & $0.34 \pm 0.21$ & $0.47 \pm 0.23$ \\
\hline \multirow{3}{*}{ 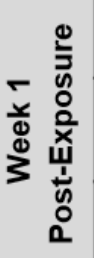 } & DPM 50 & $-0.05 \pm 0.16$ & $0.22 \pm 0.19$ & $0.63 \pm 0.26$ & $0.17 \pm 0.21$ & $0.36 \pm 0.34$ \\
\hline & SIL 233 & $4.25 \pm 0.91^{*}$ & $2.29 \pm 0.51^{*}$ & $8.73 \pm 2.19^{*}$ & $5.28 \pm 1.18^{*}$ & $0.30 \pm 0.17$ \\
\hline & DS $50 / 233$ & $2.68 \pm 0.61^{*}$ & $3.30 \pm 0.80^{*}$ & $13.75 \pm 3.63^{\star}$ & $5.51 \pm 0.57^{*}$ & $0.17 \pm 0.20$ \\
\hline \multirow{3}{*}{ 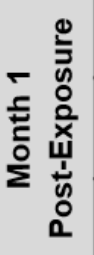 } & DPM 50 & $-0.11 \pm 0.17$ & $-0.16 \pm 0.21$ & $-0.14 \pm 0.14$ & $0.37 \pm 0.24$ & $-0.09 \pm 0.37$ \\
\hline & SIL 233 & $3.57 \pm 0.95^{*}$ & $2.45 \pm 0.83^{*}$ & $8.08 \pm 2.59^{*}$ & $10.52 \pm 5.12^{*}$ & $0.82 \pm 0.44$ \\
\hline & DS $50 / 233$ & $3.34 \pm 0.59^{*}$ & $2.54 \pm 0.51^{*}$ & $10.06 \pm 2.11^{*}$ & $6.80 \pm 1.73^{8}$ & $0.49 \pm 0.15$ \\
\hline \multirow{3}{*}{ 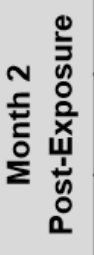 } & DPM 50 & $-0.05 \pm 0.11$ & $0.80 \pm 0.51$ & $1.16 \pm 0.66$ & $0.51 \pm 0.46$ & $0.56 \pm 0.39$ \\
\hline & SIL 233 & $1.67 \pm 0.21^{*}$ & $3.98 \pm 0.69 *$ & $5.63 \pm 0.92^{*}$ & $3.00 \pm 0.90^{*}$ & $2.30 \pm 0.51^{*}$ \\
\hline & DS $50 / 233$ & $1.30 \pm 0.35^{*}$ & $3.19 \pm 0.86^{*}$ & $4.91 \pm 1.22^{*}$ & $1.74 \pm 0.59$ & $1.37 \pm 0.83$ \\
\hline \multirow{3}{*}{ 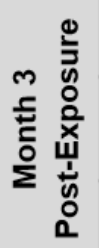 } & DPM 50 & $0.01 \pm 0.09$ & $0.11 \pm 0.15$ & $0.31 \pm 0.39$ & $-0.20 \pm 0.15$ & $-0.11 \pm 0.13$ \\
\hline & SIL 233 & $3.35 \pm 0.27^{*}$ & $4.22 \pm 1.05^{+}$ & $12.26 \pm 3.33^{+}$ & $2.70 \pm 0.74^{*}$ & $0.56 \pm 0.23$ \\
\hline & DS $50 / 233$ & $3.18 \pm 0.88^{*}$ & $2.25 \pm 0.45^{*}$ & $5.29 \pm 0.84^{*}$ & $3.24 \pm 0.57^{*}$ & $0.21 \pm 0.19$ \\
\hline
\end{tabular}

All data are expressed as mean fold change over control \pm standard error. *Different from control and DPM $50 \mu \mathrm{g}$; \& different from DPM $50 \mu \mathrm{g}$; + different from all other groups. Statistical significance is $p \leq 0.05$. 
Table 3: Lymphocyte Phenotype Determination by Flow Cytometry in the MLN Excised from Sprague-Dawley Rats Exposed to DPM, SIL, or DPM and SIL Combined

\begin{tabular}{|c|c|c|c|c|c|c|}
\hline \multirow{4}{*}{ 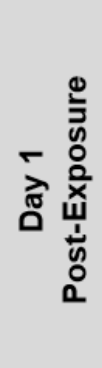 } & $\begin{array}{c}\text { Exposure } \\
(\mu g)\end{array}$ & $\begin{array}{c}\text { Total } \\
\text { Lymphocytes }\end{array}$ & T Cells & $\mathrm{CD}^{+} \mathrm{T}$ Cells & CD8 ${ }^{+}$T Cells & B Cells \\
\hline & DPM 50 & $0.09 \pm 0.12$ & $0.18 \pm 0.15$ & $0.17 \pm 0.14$ & $0.26 \pm 0.18$ & $-0.01 \pm 0.09$ \\
\hline & SIL 233 & $0.24 \pm 0.12$ & $0.24 \pm 0.12$ & $0.17 \pm 0.12$ & $0.35 \pm 0.15$ & $0.22 \pm 0.11$ \\
\hline & DS $50 / 233$ & $0.48 \pm 0.20$ & $0.53 \pm 0.25$ & $0.45 \pm 0.23$ & $0.76 \pm 0.32$ & $0.43 \pm 0.18$ \\
\hline \multirow{3}{*}{ 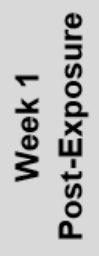 } & DPM 50 & $0.35 \pm 0.14$ & $0.39 \pm 0.17$ & $0.44 \pm 0.19$ & $0.36 \pm 0.17$ & $0.29 \pm 0.13$ \\
\hline & SIL 233 & $1.24 \pm 0.10^{*}$ & $1.50 \pm 0.11^{*}$ & $1.44 \pm 0.12^{*}$ & $1.73 \pm 0.24^{*}$ & $0.99 \pm 0.11^{*}$ \\
\hline & DS $50 / 233$ & $1.37 \pm 0.23^{*}$ & $1.56 \pm 0.23^{*}$ & $1.53 \pm 0.23^{*}$ & $1.58 \pm 0.28^{*}$ & $0.99 \pm 0.21 *$ \\
\hline \multirow{3}{*}{ 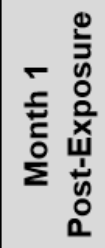 } & DPM 50 & $-0.05 \pm 0.08$ & $0.01 \pm 0.08$ & $0.04 \pm 0.09$ & $-0.04 \pm 0.09$ & $-0.10 \pm 0.09$ \\
\hline & SIL 233 & $0.70 \pm 0.24^{*}$ & $1.00 \pm 0.31^{*}$ & $0.94 \pm 0.31^{*}$ & $1.19 \pm 0.34^{*}$ & $0.53 \pm 0.19$ * \\
\hline & DS 50/233 & $0.87 \pm 0.15^{\star}$ & $1.36 \pm 0.25^{*}$ & $1.17 \pm 0.26^{*}$ & $1.71 \pm 0.32^{*}$ & $0.60 \pm 0.11^{*}$ \\
\hline \multirow{3}{*}{ 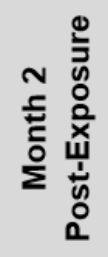 } & DPM 50 & $0.02 \pm 0.12$ & $0.10 \pm 0.13$ & $0.18 \pm 0.12$ & $0.003 \pm 0.15$ & $0.03 \pm 0.12$ \\
\hline & SIL 233 & $2.91 \pm 0.43^{\star}$ & $3.79 \pm 0.59^{*}$ & $3.76 \pm 0.66^{*}$ & $4.07 \pm 0.58^{*}$ & $2.98 \pm 0.43^{*}$ \\
\hline & DS $50 / 233$ & $3.23 \pm 0.26^{*}$ & $3.89 \pm 0.33^{*}$ & $3.66 \pm 0.33^{*}$ & $4.62 \pm 0.48^{*}$ & $3.52 \pm 0.41$ * \\
\hline \multirow{3}{*}{ 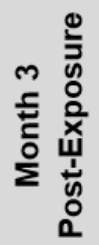 } & DPM 50 & $0.11 \pm 0.04$ & $0.02 \pm 0.06$ & $0.09 \pm 0.07$ & $-0.14 \pm 0.12$ & $0.15 \pm 0.05$ \\
\hline & SIL 233 & $4.98 \pm 1.17^{*}$ & $6.02 \pm 1.38^{*}$ & $5.17 \pm 1.11^{*}$ & $7.97 \pm 1.89 *$ & $4.46 \pm 1.15^{\star}$ \\
\hline & DS 50/233 & $4.74 \pm 0.70$ * & $5.92 \pm 0.90 *$ & $5.30 \pm 0.71^{*}$ & $6.84 \pm 1.41^{*}$ & $4.34 \pm 0.71^{*}$ \\
\hline
\end{tabular}

All data are shown as mean fold change over control \pm standard error. * Different from control and DPM $50 \mu \mathrm{g}$. Statistical significance is $p \leq 0.05$. 
Table 4: Severity and Incidence Scores for Left Lung Lobes of SpragueDawley Rats Exposed to DPM, SIL, or DPM and SIL Combined in Varying Doses

\begin{tabular}{|c|c|c|c|c|c|c|c|}
\hline $\begin{array}{c}\text { Exposure } \\
\text { Group } \\
(\mu \mathrm{g})\end{array}$ & $\begin{array}{l}\text { Time Post- } \\
\text { Exposure }\end{array}$ & AM & AEH & $\mathrm{BH}$ & LNI & BG & $\mathbf{F}$ \\
\hline Control & 1 week & $0.2(2 / 10) \mathrm{D}$ & 0 & $0.1(1 / 10) \mathrm{M}$ & 0 & 0 & 0 \\
\hline DPM 7.89 & 1 week & 0 & 0 & 0 & 0 & 0 & 0 \\
\hline DPM 50 & 1 week & $0.2(1 / 5) \mathrm{D}$ & 0 & 0 & 0 & 0 & 0 \\
\hline SIL 233 & 1 week & $1.3(10 / 10) D^{k}$ & 0 & 0 & $0.1(1 / 10) \mathrm{M}$ & 0 & 0 \\
\hline $\begin{array}{c}\text { DS } \\
7.89 / 233\end{array}$ & 1 week & $1(5 / 5) \mathrm{D}$ & 0 & $0.2(1 / 5) \mathrm{M}$ & 0 & 0 & 0 \\
\hline DS $50 / 233$ & 1 week & $2(5 / 5) D^{i *}$ & $0.4(2 / 5) \mathrm{M}^{\mathrm{j}}$ & 0 & 0 & $0.4(2 / 5) M^{j}$ & 0 \\
\hline Control & 1 month & $0.3(3 / 10) \mathrm{D}$ & 0 & 0 & 0 & 0 & 0 \\
\hline DPM 7.89 & 1 month & $1(5 / 5) \mathrm{D}$ & 0 & 0 & 0 & 0 & 0 \\
\hline DPM 50 & 1 month & 0 & 0 & 0 & 0 & 0 & 0 \\
\hline SIL 233 & 1 month & $1.7(10 / 10) D^{d *}$ & $0.3(3 / 10) \mathrm{M}$ & 0 & 0 & 0 & $0.2(2 / 10) \mathrm{M}$ \\
\hline $\begin{array}{c}\text { DS } \\
7.89 / 233\end{array}$ & 1 month & $1.8(5 / 5) D^{d}$ & $0.4(2 / 5) \mathrm{M}$ & 0 & 0 & 0 & $0.2(1 / 5) \mathrm{M}$ \\
\hline DS $50 / 233$ & 1 month & $1.8(5 / 5) D^{d}$ & $1(5 / 5) M^{i \star}$ & 0 & 0 & $0.8(4 / 5) M^{\mathrm{b} *}$ & $0.8(4 / 5) M^{i \star \star}$ \\
\hline Control & 3 months & $0.5(5 / 10) \mathrm{D}$ & 0 & 0 & 0 & 0 & 0 \\
\hline DPM 7.89 & 3 months & $0.6(3 / 5) \mathrm{D}$ & 0 & 0 & 0 & 0 & 0 \\
\hline DPM 50 & 3 months & $0.8(4 / 5) \mathrm{D}$ & 0 & 0 & 0 & 0 & 0 \\
\hline SIL 233 & 3 months & $1.8(10 / 10) D^{k \star *}$ & $1.1(10 / 10) M^{i * *}$ & 0 & 0 & 0 & $1(8 / 10) M^{h *}$ \\
\hline $\begin{array}{c}\text { DS } \\
7.89 / 233\end{array}$ & 3 months & $2(5 / 5) D^{k * *}$ & $1(5 / 5) M^{h}$ & 0 & 0 & 0 & $1(5 / 5) M^{\mathrm{h}}$ \\
\hline DS $50 / 233$ & 3 months & $1.4(5 / 5) \mathrm{D}$ & $1.4(5 / 5) M^{i \star \star}$ & 0 & 0 & $0.6(3 / 5) M^{b *}$ & $0.8(3 / 5) M$ \\
\hline
\end{tabular}

Lung tissue was analyzed and scored for accumulation of alveolar macrophages as an index of inflammation (AM), alveolar epithelial hyperplasia (AEH), bronchus associated lymphoid tissue hyperplasia $(\mathrm{BH})$, lymphoid nodules in the interstitium (LNI), brown granules in alveolar macrophages (BG; presence of DPM particles), fibrosis in alveolar septae $(F)$ ( $n=5$ or 10 per group per time point). Severity was scored as $0-5: 0=$ normal, $1=$ minimal $/$ slight, $2=$ mild, $3=$ moderate, $4=$ marked, and $5=$ severe. Incidence was noted as Focal (F), Multi-focal (M), and Diffuse (D). Data are presented as means with incidence (number of animals with a positive score per total animals) in parentheses. b ${ }^{\text {D }}$ ifferent from all other groups; ddifferent from control and DPM $50 \mu \mathrm{g}$ only; ' different from control only; idifferent from control, DPM $7.89 \mu \mathrm{g}$, and DPM $50 \mu \mathrm{g}$ group; jifferent from control and SIL $233 \mu \mathrm{g}$ group; ${ }^{k}$ different from control and DPM $7.89 \mu \mathrm{g}$ group. Note: all notations indicate a $p$ value $<0.05$ except where asterisks indicate ${ }^{*} p<0.01$ and ${ }^{* *} p<0.05$ for all groups except control where $p<0.01$. 
Table 5: Lymphocyte Phenotype Determination by Flow Cytometry in the BALF Recovered from Sprague-Dawley Rats Exposed to DPM, SIL, or DPM and SIL Combined with LM

\begin{tabular}{|c|c|c|c|c|c|c|}
\hline \multirow{4}{*}{ 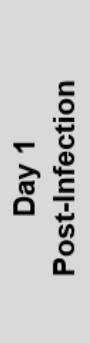 } & Exposure $(\mu \mathrm{g})$ & $\begin{array}{c}\text { Total } \\
\text { Lymphocytes }\end{array}$ & T Cells & CD4 $^{+} \mathrm{T}$ Cells & CD8 ${ }^{+}$T Cells & B Cells \\
\hline & DPM 50 & $0.19 \pm 0.22$ & $-0.05 \pm 0.20$ & $-0.04 \pm 0.20$ & $-0.02 \pm 0.27$ & $0.17 \pm 0.32$ \\
\hline & SIL 233 & $0.92 \pm 0.30 @$ & $0.90 \pm 0.40$ & $0.89 \pm 0.37$ & $0.88 \pm 0.51$ & $0.57 \pm 0.40$ \\
\hline & DS 50/233 & $0.52 \pm 0.23$ & $0.50 \pm 0.26$ & $0.48 \pm 0.27$ & $0.71 \pm 0.38$ & $0.09 \pm 0.27$ \\
\hline \multirow{3}{*}{ m } & DPM 50 & $-0.07 \pm 0.17$ & $-0.31 \pm 0.14$ & $-0.17 \pm 0.18$ & $-0.35 \pm 0.20$ & $-0.15 \pm 0.11$ \\
\hline & SIL 233 & $-0.40 \pm 0.15$ & $-0.48 \pm 0.09 @$ & $-0.30 \pm 0.08$ & $-0.53 \pm 0.11$ & $-0.40 \pm 0.11$ \\
\hline & DS $50 / 233$ & $-0.54 \pm 0.07$ & $-0.59 \pm 0.05 @$ & $-0.33 \pm 0.07$ & $-0.70 \pm 0.04 @$ & $-0.60 \pm 0.05 @$ \\
\hline \multirow{3}{*}{ 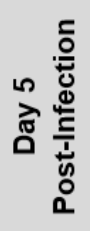 } & DPM 50 & $0.001 \pm 0.21$ & $-0.04 \pm 0.15$ & $-0.18 \pm 0.14$ & $0.03 \pm 0.16$ & $0.34 \pm 0.19$ \\
\hline & SIL 233 & $-0.25 \pm 0.17$ & $-0.27 \pm 0.13$ & $-0.40 \pm 0.10$ & $-0.20 \pm 0.14$ & $-0.07 \pm 0.24$ \\
\hline & DS 50/233 & $-0.19 \pm 0.21$ & $-0.15 \pm 0.24$ & $-0.23 \pm 0.14$ & $-0.12 \pm 0.31$ & $-0.13 \pm 0.15$ \\
\hline \multirow{3}{*}{ 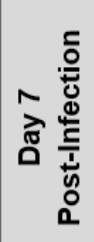 } & DPM 50 & $-0.03 \pm 0.12$ & $0.05 \pm 0.14$ & $-0.02 \pm 0.12$ & $-0.004 \pm 0.14$ & $-0.13 \pm 0.12$ \\
\hline & SIL 233 & $-0.15 \pm 0.10$ & $-0.08 \pm 0.13$ & $-0.17 \pm 0.10$ & $-0.09 \pm 0.17$ & $0.10 \pm 0.21$ \\
\hline & DS 50/233 & $-0.20 \pm 0.10$ & $-0.19 \pm 0.11$ & $-0.27 \pm 0.11$ & $-0.17 \pm 0.15$ & $-0.21 \pm 0.13$ \\
\hline \multirow{3}{*}{ 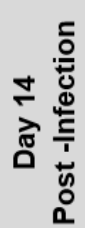 } & DPM 50 & $0.09 \pm 0.14$ & $0.17 \pm 0.21$ & $0.22 \pm 0.25$ & $0.18 \pm 0.17$ & $0.27 \pm 0.26$ \\
\hline & SIL 233 & $0.89 \pm 0.20^{*}$ & $0.65 \pm 0.23$ & $1.01 \pm 0.19$ & $0.89 \pm 0.36$ & $-0.05 \pm 0.12$ \\
\hline & DS 50/233 & $0.95 \pm 0.26^{*}$ & $0.80 \pm 0.29$ & $1.46 \pm 0.54^{\star}$ & $0.67 \pm 0.23$ & $0.002 \pm 0.17$ \\
\hline
\end{tabular}

All data are shown as mean fold change over control \pm standard error. *Different from control and DPM $50 \mu \mathrm{g}$; ${ }^{\circledR}$ different from control. Statistical significance is $p \leq 0.05$. 
Table 6: Lymphocyte Phenotype Determination by Flow Cytometry in the MLN Excised from Sprague-Dawley Rats Exposed to DPM, SIL, or DPM and SIL Combined with LM

\begin{tabular}{|c|c|c|c|c|c|c|}
\hline \multirow{4}{*}{ 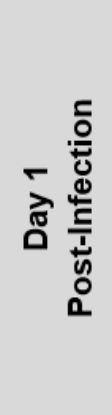 } & $\begin{array}{l}\text { Exposure } \\
(\mu \mathrm{g})\end{array}$ & $\begin{array}{c}\text { Total } \\
\text { Lymphocytes }\end{array}$ & T Cells & CD4 $^{+}$T Cells & CD8 ${ }^{+}$T Cells & B Cells \\
\hline & DPM 50 & $0.21 \pm 0.21$ & $0.18 \pm 0.21$ & $0.19 \pm 0.20$ & $0.12 \pm 0.22$ & $0.26 \pm 0.23$ \\
\hline & SIL 233 & $0.80 \pm 0.26 @$ & $0.76 \pm 0.24^{*}$ & $0.70 \pm 0.22 @$ & $0.59 \pm 0.22$ & $0.69 \pm 0.22$ \\
\hline & DS 50/233 & $0.72 \pm 0.18 @$ & $0.82 \pm 0.19 @$ & $0.88 \pm 0.19^{*}$ & $0.66 \pm 0.18$ & $0.66 \pm 0.18$ \\
\hline \multirow{3}{*}{ m } & DPM 50 & $-0.16 \pm 0.06$ & $-0.18 \pm 0.07$ & $-0.08 \pm 0.08$ & $-0.30 \pm 0.07 @$ & $-0.19 \pm 0.06$ \\
\hline & SIL 233 & $0.26 \pm 0.10^{+}$ & $0.14 \pm 0.09^{\wedge}$ & $0.24 \pm 0.10$ & $-0.04 \pm 0.08^{\wedge}$ & $0.15 \pm 0.09$ \\
\hline & DS 50/233 & $-0.08 \pm 0.08$ & $-0.18 \pm 0.03$ & $-0.04 \pm 0.02$ & $-0.36 \pm 0.04 @$ & $-0.03 \pm 0.11$ \\
\hline \multirow{3}{*}{ n } & DPM 50 & $0.40 \pm 0.16$ & $0.35 \pm 0.15$ & $0.33 \pm 0.15$ & $0.38 \pm 0.15$ & $0.52 \pm 0.22$ \\
\hline & SIL 233 & $0.04 \pm 0.10$ & $0.03 \pm 0.10$ & $0.01 \pm 0.10$ & $0.04 \pm 0.12$ & $0.01 \pm 0.09$ \\
\hline & DS 50/233 & $0.32 \pm 0.14$ & $0.28 \pm 0.13$ & $0.28 \pm 0.14$ & $0.24 \pm 0.12$ & $0.37 \pm 0.21$ \\
\hline \multirow{3}{*}{ 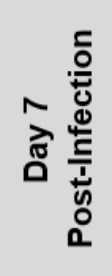 } & DPM 50 & $0.60 \pm 0.27$ & $0.72 \pm 0.31$ & $0.76 \pm 0.31$ & $0.66 \pm 0.30$ & $0.50 \pm 0.25$ \\
\hline & SIL 233 & $0.30 \pm 0.20$ & $0.34 \pm 0.23$ & $0.41 \pm 0.25$ & $0.24 \pm 0.20$ & $0.25 \pm 0.18$ \\
\hline & DS 50/233 & $0.40 \pm 0.15$ & $0.40 \pm 0.16$ & $0.47 \pm 0.15$ & $0.31 \pm 0.19$ & $0.40 \pm 0.16$ \\
\hline \multirow{3}{*}{ 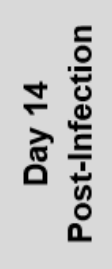 } & DPM 50 & $0.02 \pm 0.28$ & $-0.06 \pm 0.27$ & $-0.06 \pm 0.27$ & $-0.04 \pm 0.29$ & $0.18 \pm 0.36$ \\
\hline & SIL 233 & $0.03 \pm 0.18$ & $0.03 \pm 0.19$ & $0.01 \pm 0.16$ & $0.12 \pm 0.26$ & $0.12 \pm 0.24$ \\
\hline & DS 50/233 & $0.21 \pm 0.26$ & $0.21 \pm 0.27$ & $0.27 \pm 0.30$ & $0.15 \pm 0.24$ & $0.27 \pm 0.31$ \\
\hline
\end{tabular}

All data are shown as mean fold change over control \pm standard error. *Different from control and DPM $50 \mu \mathrm{g}$; @ different from control; ^ different from DPM $50 \mu \mathrm{g}$ and DS 50/233 $\mu \mathrm{g}$ groups; "different from SIL $233 \mu \mathrm{g}$; +different from all other groups. Statistical significance is $p \leq 0.05$. 


\section{Figure 1}
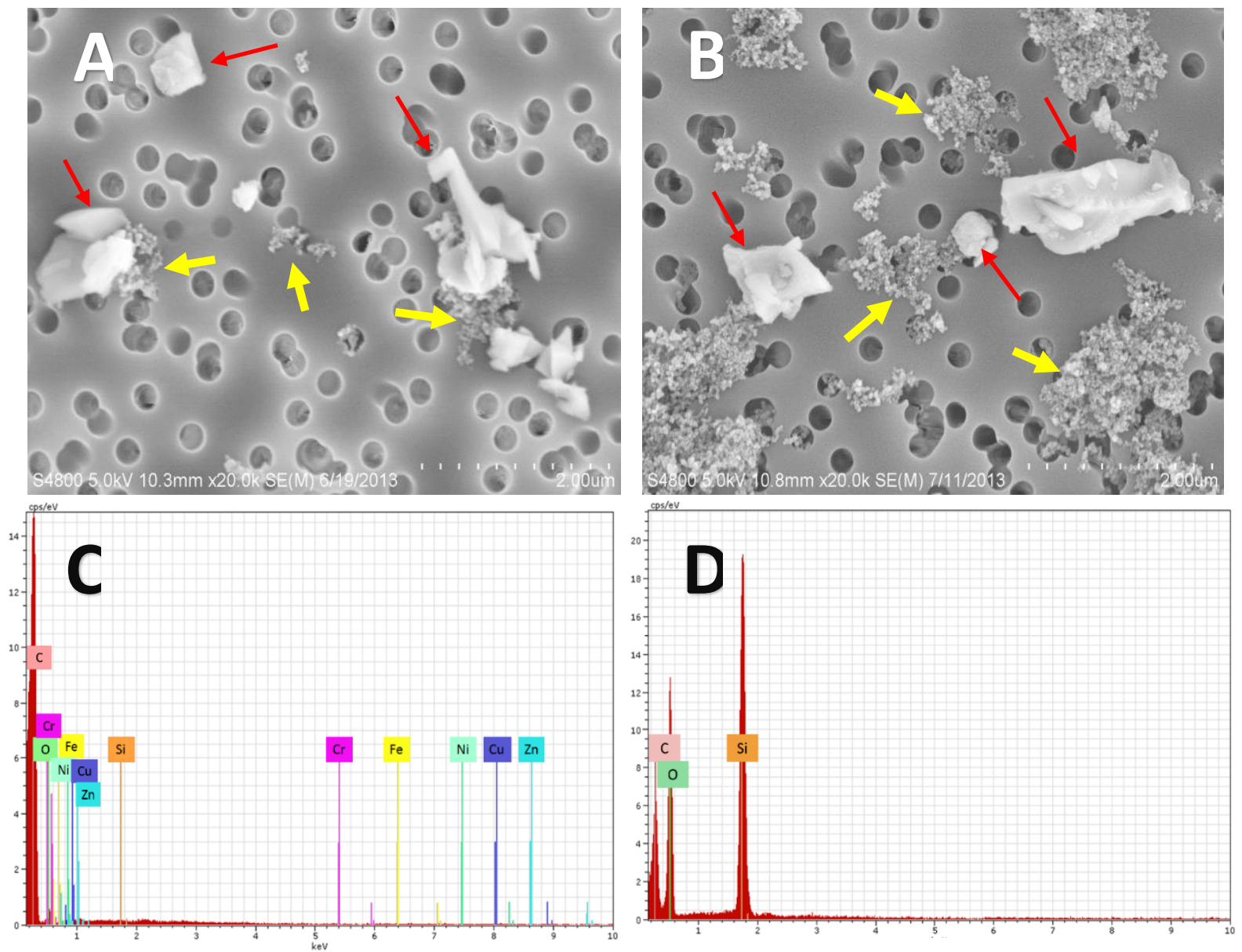

Figure 1: FESEM images of particles. (A) $233 \mu \mathrm{g}$ SIL and $7.89 \mu \mathrm{g}$ DPM in combination prepared in PBS solution, diluted 1:10 in PBS, then dried onto a filter under 20,000x magnification (scale bar $=2 \mu \mathrm{m}$ with 10 segments of $0.2 \mu \mathrm{m}$ each) (B) $233 \mu \mathrm{g} \mathrm{SIL}$ and $50 \mu \mathrm{g}$ DPM prepared in PBS solution, diluted 1:10 in PBS, then dried onto a filter in PBS solution under $20,000 \times$ magnification (scale bar $=2 \mu \mathrm{m}$ with 10 segments of $0.2 \mu \mathrm{m}$ each). Red arrows indicate SIL and yellow arrows indicate DPM. (C) EDX of DPM particles pictured in the FESEM above at $20 \mathrm{keV}$. Labeled spectral peaks for elements primarily present in the samples appear in red (carbon). All other labeled peaks are elements commonly found in DPM but present only in trace amounts in this sample (Chromium, Copper, Iron, Nickel, Oxygen, Silicon, and Zinc). (D) EDX of SIL particles pictured in FESEM above at $20 \mathrm{keV}$. 
Figure 2

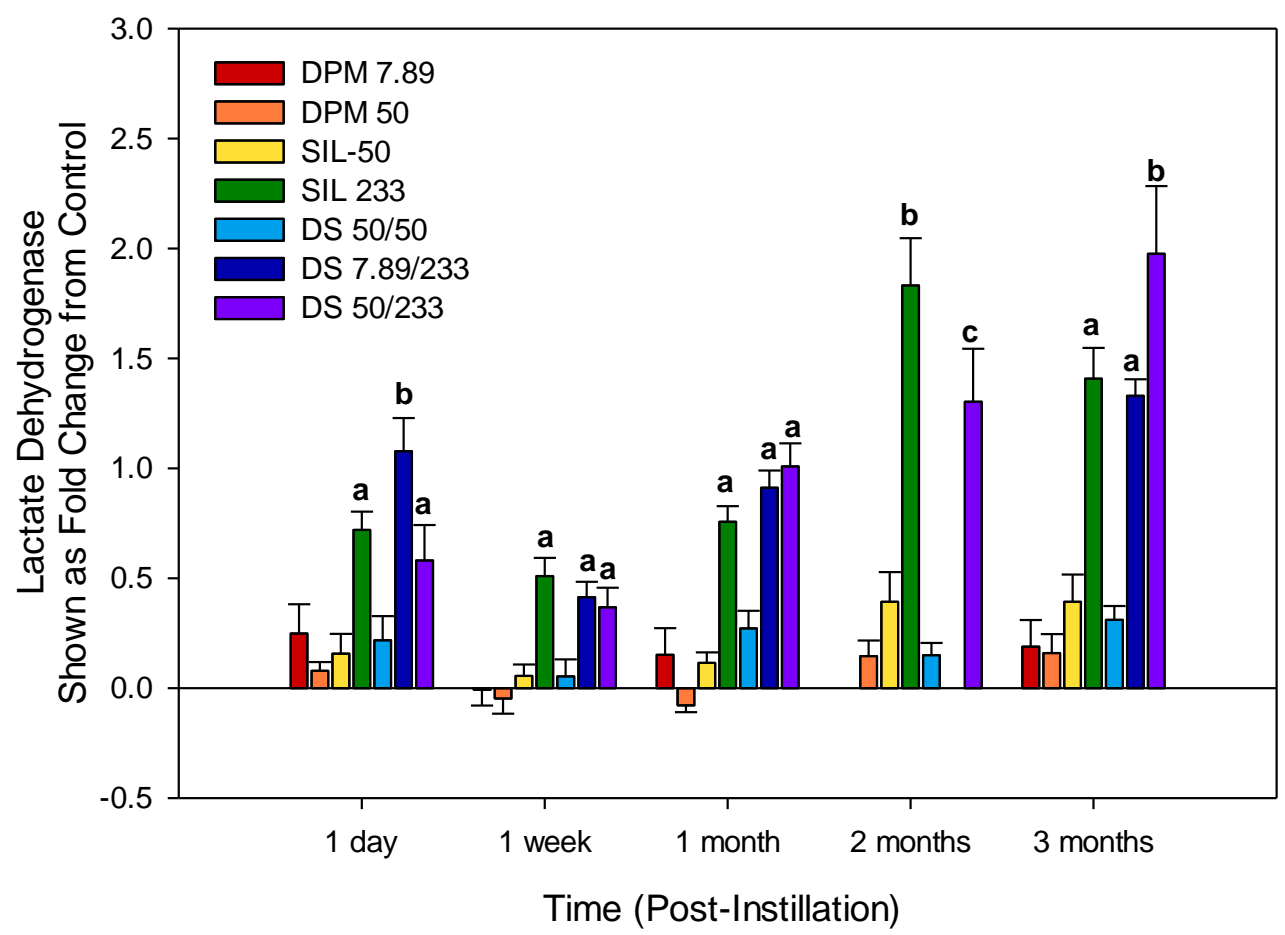

Figure 2: LDH activity in BALF after exposure to DPM, SIL, or a combination of DPM and SIL (DS) at indicated doses $(\mu \mathrm{g})$. Data are shown as mean fold change over control

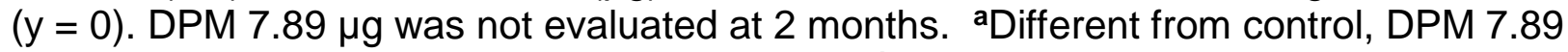
$\mu \mathrm{g}$, DPM $50 \mu \mathrm{g}$, SIL $50 \mu \mathrm{g}$, DS 50/50 $\mu \mathrm{g}$ groups; ' different from all other groups; 'different from control, DPM $50 \mu \mathrm{g}$, SIL $50 \mu \mathrm{g}$, and DS 50/50 $\mu \mathrm{g}$ groups. Statistical significance is $p \leq 0.05$. 
Figure 3
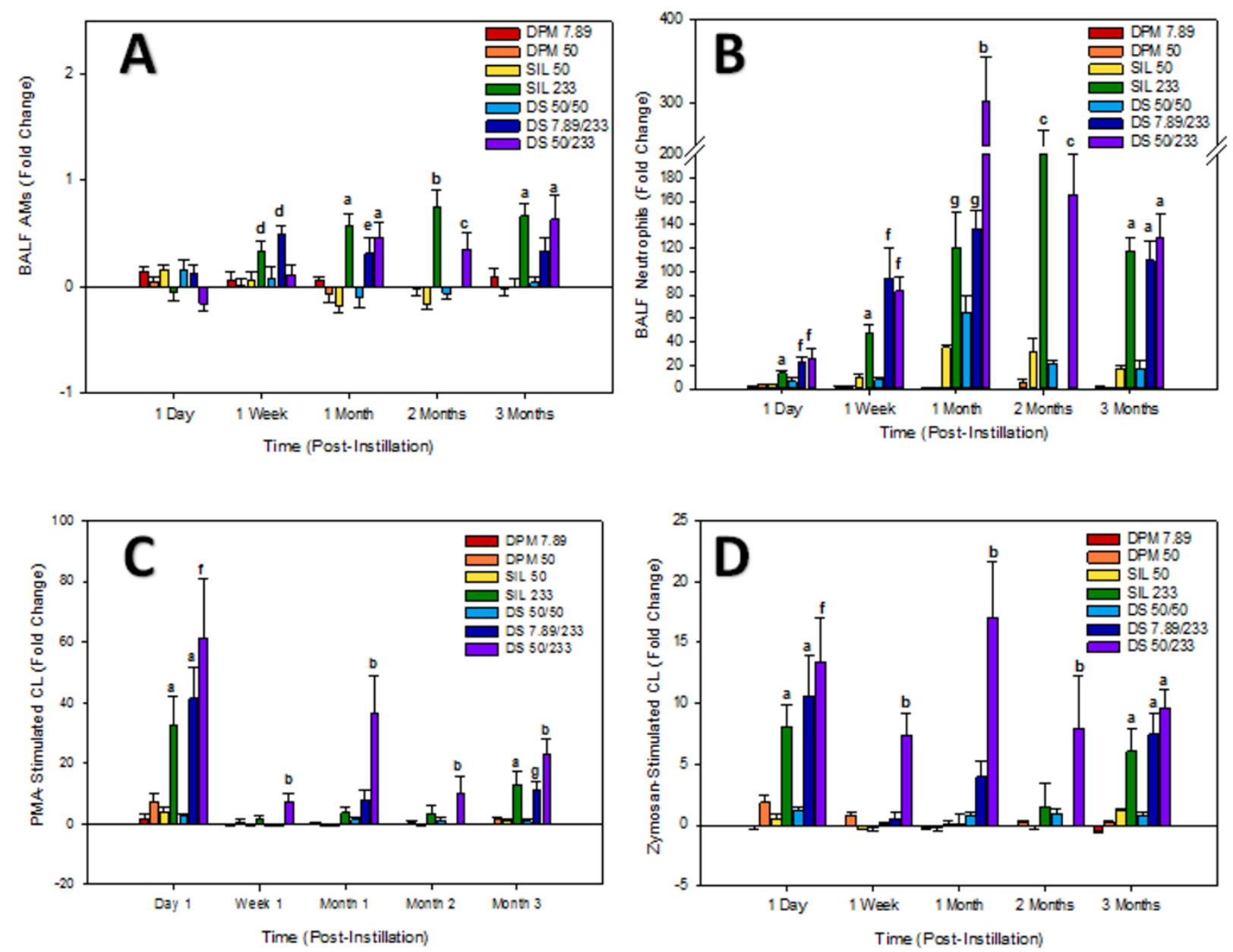

Figure 3: Total AMs (A) and neutrophils (B) in the BALF following exposure to DPM, SIL, or DS at doses indicated. Production of oxidants by total phagocytes stimulated with PMA (C) or by AMs only stimulated by zymosan (D) measured by chemiluminescence following exposure to DPM, SIL or DS at doses indicated. DPM $7.89 \mu \mathrm{g}$ was not evaluated at 2 months. All data are shown as a mean fold change over

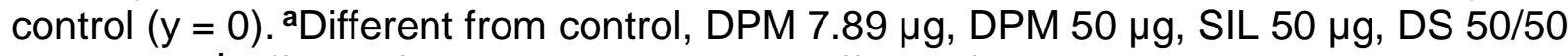
$\mu \mathrm{g}$ groups; ${ }^{b}$ different from all other groups; ' ${ }^{c}$ different from control, DPM $7.89 \mu \mathrm{g}$, DPM $50 \mu \mathrm{g}$, SIL $50 \mu \mathrm{g}$, and DS 50/50 $\mu \mathrm{g}$ groups; ddifferent from control and DPM $50 \mu \mathrm{g}$ only; edifferent from control , DPM $50 \mu \mathrm{g}$, and SIL $50 \mu \mathrm{g}$ groups; ${ }^{\mathrm{f}}$ different from control, DPM $7.89 \mu \mathrm{g}$, DPM $50 \mu \mathrm{g}$, SIL $50 \mu \mathrm{g}$, and DS 50/50 $\mu \mathrm{g}$; 9different from control, DPM $7.89 \mu \mathrm{g}$, DPM $50 \mu \mathrm{g}$, and SIL $50 \mu \mathrm{g}$ groups. Statistical significance measured as $p \leq 0.05$. 
Figure 4
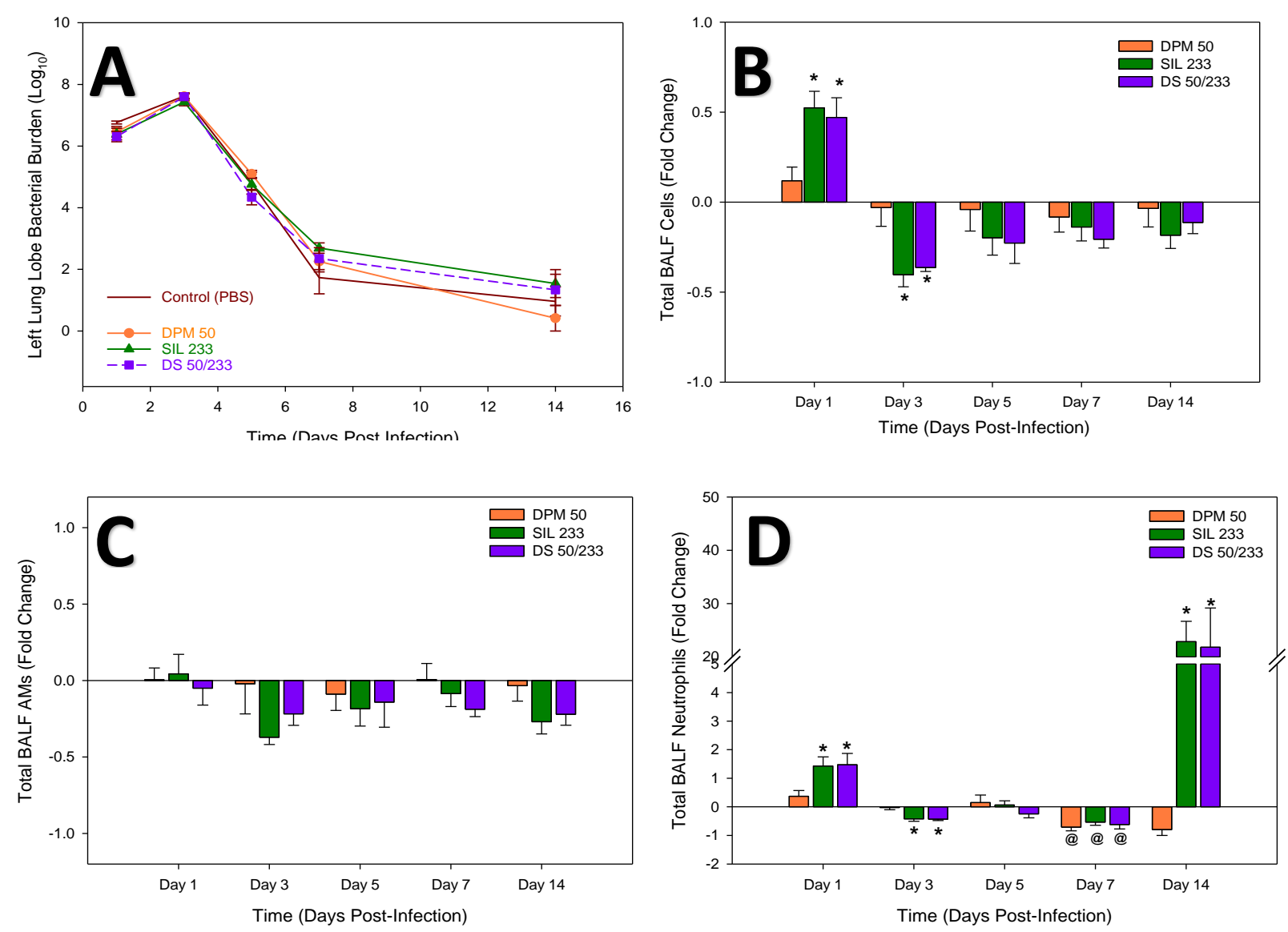

Figure 4: (A) Bacterial burden in the left lung over the time-course. Data is shown on a $\log _{10}$ scale. Total cells (B), total AMs (C), and total neutrophils (D) recovered by lavage following exposure to DPM, SIL, or DS at doses indicated. (B-D) Data are shown as a mean fold change over control $(y=0)$. *Different from control and DPM $50 \mu \mathrm{g}$; ${ }^{\circledR}$ different from control. Statistical significance is $p \leq 0.05$. 


\section{Chapter 3 - Study 2}

The following represents data that will be submitted to a peer-reviewed journal for publication. 
Pulmonary Toxicity following Acute Co-Exposures to Crystalline Silica and Diesel Emission Particulates or Carbon in Rats: differentiating particle-specific versus particle load-effects

Breanne Y. Farris ${ }^{1,2}$, Robert R. Mercer ${ }^{1}$, James M. Antonini ${ }^{1,2}$, Mark W. Barger ${ }^{1}$, Bean T. Chen ${ }^{1}$, Sherri Friend ${ }^{1}$, Michael L. Kashon ${ }^{1}$, Jeffrey S. Fedan ${ }^{1,2}$, and Jenny R. Roberts ${ }^{1,2}$.

${ }^{1}$ National Institute for Occupational Safety and Health, Morgantown, WV

${ }^{2}$ West Virginia University, Morgantown, WV 


\section{Abstract}

The effects of acute pulmonary co-exposures to silica and diesel particulate matter (DPM), which may occur in various occupations including the mining and construction industries, were investigated in vivo. Rats were exposed by intratracheal instillation (IT) to silica (SIL) $233 \mu \mathrm{g}$, DPM $233 \mu \mathrm{g}$, carbon black (CB) $233 \mu \mathrm{g}$, DPM and SIL (DPM/SIL) combined, CB and SIL (CB/SIL) combined, or to PBS alone (vehicle control). At 1 day, 1 week, 1 month, 2 months, and 3 months post-exposure, bronchoalveolar lavage and histopathology were performed to assess lung injury, inflammation, and immune response. Although SIL caused enhanced parameters of inflammation and injury at all times-points, DPM exposure alone caused increased lactate dehydrogenase activity only at the early time-points (1 day, 1 week) post-exposure when compared to control. Parameters of increased injury were also observed in the CB group compared to control and compared to DPM and control for inflammation at day 1; however, these effects were transient and subsided by 1 week. DPM combined with SIL increased oxidant production by phagocytes compared with all other groups at all time-points post exposure, and increased markers of inflammation, injury, the influx of lymphocytes in the lung, the proliferation of lymphocytes in the mediastinal lymph nodes (MLN), and the incidence of epithelial hyperplasia in lung tissue, scored as minimal, at later time-points compared to exposure to SIL alone. The combination of CB with SIL caused increased macrophage oxidant production at 1 day compared with control and DPM groups, and 1 week and 1 month compared with all individual particle dose groups, which resolved by 2 months post-exposure. Additionally, CB combined with SIL resulted in increased markers of inflammation and injury at all time-points following exposure; however, these effects were primarily attributed to the effects of SIL, particularly at later time-points following exposure. To assess susceptibility to lung infection following co-exposure, rats were exposed to particles, as described above, 1 week before intratracheal inoculation with $5 \times 10^{5}$ Listeria monocytogenes. At 1, 3, 5, 7, and 14 days following infection, pulmonary immune response and bacterial clearance from the lung were evaluated. Co-exposure to CB and SIL increased bacterial clearance at day 1 compared with control and CB only. At day 3, animals exposed to SIL alone or in combination with CB or DPM cleared the infection to a greater degree than those exposed to DPM alone. No differences in clearance rate occurred beyond day 3 and all groups effectively resolved the infection by day 14. The study showed that DPM had the capacity to increase SIL-induced lung injury, inflammation and oxidant production to a greater degree than equal doses of CB and SIL, indicating these effects are due to properties inherent to DPM rather than particle load. Alternately, tissue remodeling at later time points, indicated by increased incidence of tissue 
remodeling proteins in the lung and fibrosis, correlated better with particle load and the presence of SIL following co-exposures. Additionally, particle-specific effects were observed in the susceptibility model whereby DPM alone transiently reduced innate immune response to a pathogen, and SIL appeared to enhance the bacterial clearance at early time points postinfection; however, effects on susceptibility to infection did not occur during the adaptive phase of the response. Overall, the study showed that in co-exposure to SIL and DPM, total particle load and the presence of SIL contribute to toxicity in the lung, and in addition, DPM caused particle specific effects that significantly altered the toxicological outcomes beyond that of just particle load.

\section{Introduction}

Many workers in industrial occupations may be at risk for exposure to respirable particles as a part of their regular duties. This is particularly true in industries that utilize heavy machinery powered by diesel engines. Diesel exhaust releases particulates into the air, which are respirable in size. Furthermore, occupations that involve the redistribution of earth or the extraction of natural resources including construction, excavation, tunneling, agriculture, metal and non-metal mining, and the oil and gas industry may not only utilize this equipment during operations, but depending on the industry or operation, a co-exposure with quartz or crystalline silica $\left(\mathrm{SiO}_{2}\right)$ that is ubiquitous in nature may also occur. Field studies performed by researchers at the National Institute for Occupational Safety and Health (NIOSH) identified both of these particulates as potential concerns for occupational exposure [461]. Furthermore, since dieselpowered equipment has gained popularity as a mechanical mechanism of extracting mineral resources underground, these types of co-exposures are common in many mining environments, and have the potential to create particulate exposures that are greater in concentration due to the confined working environments encountered underground [403]. While the toxicities of these two particulates have been studied in detail as individual exposures, there are very few in vivo studies examining the pulmonary effects of these particulates in combination or at doses that are comparable to measurements collected during occupational activities.

Previously we have shown that exposure to DPM at doses derived from particulate measures collected during different mining operations were minimally toxic as an individual exposure; however, when in combination with crystalline silica, the presence of DPM significantly enhanced parameters of inflammation, oxidant production, and the onset of fibrotic 
tissue changes in the lung as compared to silica (SIL) alone. Furthermore, DPM in combination with SIL was shown to alter some immunological responses observed individually due to SIL exposure [539]. The study however, did not delineate whether specific properties of DPM contributed to these responses or whether the presence of additional particle, or overall particle load, influenced these effects. The goal of the current study was to evaluate whether those effects are particle- or particle load-specific. DPM is a complex mixture of aromatic hydrocarbons, metals, nitrates, sulfates, and other chemicals complexed to a carbon core. For this purpose, carbon black (Printe $x^{\circledR}$ 90; CB) was used as a reference particle to control for load effects versus DPM-specific effects, as it represents the core material of DPM without the constituents of DPM frequently associated with toxicity $[277,281,298,326,332,333,335,467$, 481].

Doses for the current study were based on field measurements of $\mathrm{SiO}_{2}$ taken during active surface-level mining operations [486]. Equivalent doses of DPM and CB were administered in an effort to determine the toxicological effects due specifically to a particular particle (SIL, DPM, or CB). Further, combinations of CB or DPM with SIL were administered to determine load versus particle specific effects that may result in a co-exposure scenario. Similar to the previous study [539], a single acute bolus dose of SIL, DPM, CB, a combination of DPM and SIL (DPM/SIL), or a combination of CB and SIL (CB/SIL), were administered to SpragueDawley rats by a single intratracheal instillation (IT). Parameters of lung injury, inflammation, histopathology, and immune responses were evaluated over a three-month time-course. In addition, susceptibility to pulmonary infection following exposure to the particles individually and in combination was also evaluated to further assess alterations in immune response following particle exposure.

\section{Methods}

Particles. Particles in these studies were used as surrogates to represent work site exposures. The surrogate particle used for $\mathrm{SiO}_{2}$ was $\alpha$-quartz crystalline silica (SIL; MIN-U-SIL® 5, US SILICA; Berkeley Springs, WV). DPM was obtained from the National Institute of Standards and Technology (NIST) as a commercially available NIST Standard Reference Material (SRM) 2975 (referred to as DPM in this study). SRM 2975 was originally collected from an industrial forklift in the 1990s [540]. Carbon black (CB; Printex ${ }^{\circledR} 90$ from Degussa-Hüls, Parsippany, NJ) was used as a reference material and control for effects related to particle load. The particle was selected 
based on composition, as carbon black comprises the core material of DPM, and size of primary particles derived from field emission scanning electron micrographs.

Dose Determination. Doses were derived from field measurements of $\mathrm{SiO}_{2}$ and applied to the equation below (EQ1) to quantify the amount of particle that would deposit in the lungs of an average male worker doing moderate work for 12 hours per day for a period of 14 continuous days using pulmonary deposition efficacy for each particle. Deposition efficacy was derived from a software-based algorithm that considers particle characteristics including shape and density [541]. This dose was then normalized to the surface area of both the worker's and rat's lungs to obtain dose for in vivo studies (EQ2). The SIL dose (233 $\mu \mathrm{g} \mathrm{SIL)} \mathrm{was} \mathrm{derived} \mathrm{from} \mathrm{the} \mathrm{highest}$ $\mathrm{SiO}_{2}$ levels measured during hydraulic fracturing site visits [486], $2.5 \mathrm{mg} / \mathrm{m}^{3}$ (2.5 $\left.\mu \mathrm{g} / \mathrm{l}\right)$ (EQ3). It was previously demonstrated that DPM in a co-exposure with SIL induced greater inflammation, oxidant production by phagocytes, and onset of pulmonary fibrosis in comparison to either particle individually. The goal of this study was to determine whether particle load induced these effects or if effects were due to DPM specifically. For this purpose, DPM or CB were administered at a dose equivalent to that of SIL to determine which particle, if either, had a greater toxic effect in a co-exposure.

\section{EQ1 - Basic equation for standard worker lung deposition:}

$=($ field concentration, $\mu \mathrm{g} / \mathrm{l}) \times($ minute ventilation, $\mathrm{l} / \mathrm{min}) \times($ exposure time per day, $\mathrm{min} / \mathrm{d})$ $x$ (total fracking days, $d$ ) $x$ (pulmonary deposition efficacy, unitless)

\section{EQ2 - Equation for normalization to rat lung surface area:}

$=$ (worker's deposited dose/average male worker lung surface area) $\mathrm{x}$ (average rat lung surface area)

\section{EQ3 - Equation for SIL Dose:}

Worker lung deposition $=(2.5 \mu \mathrm{g} / \mathrm{I}) \times(20 \mathrm{l} / \mathrm{min}) \times(720 \mathrm{~min} /$ day $) \times(14$ days $) \times(0.118)=$ $59,472 \mu \mathrm{g}$ per worker

Dose calculated $=\left(59,472 \mu \mathrm{g} / 102.2 \mathrm{~m}^{2}\right) \times\left(0.4 \mathrm{~m}^{2}\right) \approx 233 \mu \mathrm{g}$ per rat

SIL, DPM, and CB Preparation and Characterization. Particles were prepared in USP-grade phosphate-buffered sterile saline without $\mathrm{Ca}^{2+}$ or $\mathrm{Mg}^{2+}$ (PBS: AMERESCO, Solon, OH) for IT. A sonication process was used to disperse agglomerated particles. SIL was prepared as a 10 $\mathrm{mg} / \mathrm{ml}$ stock solution, DPM and CB were prepared as $2 \mathrm{mg} / \mathrm{ml}$ stock solutions. All particles were 
vortex mixed for $1 \mathrm{~min}$, followed by $1 \mathrm{~min}$ of continuous sonication (GE130PB ultrasonic processor; General Electric, Boston, MA). Aliquots of stock solution were then further diluted to $233 \mu \mathrm{g}$ SIL, $233 \mu \mathrm{g}$ DPM, $233 \mu \mathrm{g} \mathrm{CB}, 233 \mu \mathrm{g}$ DPM + $233 \mu \mathrm{g} \mathrm{SIL}$, or $233 \mu \mathrm{g}$ CB $+233 \mu \mathrm{g}$ SIL in $0.3 \mathrm{ml}$ PBS. Particles were then administered to the rats by IT as a single dose as previously described [539].

Animals. Male Sprague-Dawley [Hla:(SD) CVF] (SD) rats from Hilltop Lab Animals (Scottdale, PA), weighing approximately $300 \mathrm{~g}$ and free of viral pathogens, parasites, mycoplasmas, Helicobacter, and CAR Bacillus, were used for all studies. Rats were housed in the pathogen free, environmentally controlled, AAALAC-accredited facility. The rats were housed in ventilated polycarbonate cages, two per cage, on Alpha-Dri cellulose chips and hardwood Beta chips as bedding; they were provided HEPA-filtered air, irradiated Teklad 2918 diet, and tap water ad libitum; and were allowed to acclimate for one week before exposure. Rats were not restricted from enrichment activity

(i.e., chewing/climbing). All animal procedures used during the study were reviewed and approved by the IACUC in the Health Effects Laboratory Division (HELD) at NIOSH.

Time-Course Study Design. In Vivo Lung Injury, Inflammation, and Disease. On day 0, rats were lightly anesthetized with an intraperitoneal injection of $30-40 \mathrm{mg} / \mathrm{kg}$ sodium methohexital (Brevital; Eli Lilly, Indianapolis, IN) and intratracheally instilled with $233 \mu \mathrm{g}$ DPM, $233 \mu \mathrm{g}$ CB, $233 \mu \mathrm{g}$ SIL, $233 \mu \mathrm{g}$ DPM + $233 \mu \mathrm{g}$ SIL, $233 \mu \mathrm{g}$ CB + $233 \mu \mathrm{g}$ SIL, or vehicle control (PBS). Studies were conducted in two blocks with paired PBS and SIL controls. All particles were delivered in $0.3 \mathrm{ml}$ USP grade PBS. All animals were humanely euthanized at 1 day, 1 week, 1 month, 2 months, or 3 months following particle exposure ( $n=8 /$ group/time point for DPM, CB, DPM/SIL, and CB/SIL groups and $n=16$ for PBS and SIL groups) with an intraperitoneal injection of $100-300 \mathrm{mg} / \mathrm{kg}$ sodium pentobarbital, followed by exsanguination. The trachea was cannulated, bronchoalveolar lavage (BAL) was performed on the right lung lobes, and BAL cells (BALCs) and fluid (BALF) were retained for analysis of parameters indicative of inflammation, injury, oxidant production, and immune function. Lung injury and inflammation were evaluated as the presence of lactate dehydrogenase (LDH) activity, cytokines, and chemokines in BALF. BALCs were differentiated and phenotyped. Lymphocytes from the mediastinal lymph nodes (MLN) draining the lung were also differentiated by phenotype. Chemiluminescence assays were used to measure oxidant/free radical production by BAL phagocytes to evaluate the inflammatory response. The left lung was excised, pressure-inflated with $10 \%$ neutral-buffered formalin, and weighed with water-displacement to determine fixed lung volume. 
Histopathological assessment of lung injury, inflammation, and disease was performed, and morphometric analysis was performed to assess particulate lung burden.

Infection Study Design. Pulmonary Immune Response to Infection Following Particle Exposure. In this study, rats were exposed to $233 \mu \mathrm{g}$ DPM, $233 \mu \mathrm{g} \mathrm{CB}, 233 \mu \mathrm{g} \mathrm{SIL}, 233 \mu \mathrm{g}$ $\mathrm{DPM}+233 \mu \mathrm{g}$ SIL, or $233 \mu \mathrm{g}$ CB $+233 \mu \mathrm{g}$ SIL combined in $0.3 \mathrm{ml}$ PBS, or PBS alone (vehicle control) by intratracheal instillation as described above. One week following particle exposure, rats were intratracheally instilled with $\sim 5 \times 10^{5}$ colony forming units (cfu) of Listeria monocytogenes (strain 10403S, serotype 1; LM) in $0.5 \mathrm{ml}$ of sterile PBS. The study was conducted with the same block design as the previous study with paired PBS and SIL controls. Animals were euthanized at 1, 3, 5, 7, and 14 days following infection ( $n=8$ /dose/time point for DPM, CB, DPM/SIL, and CB/SIL groups and $n=16$ for PBS and SIL groups). BAL was performed on the right lung lobes, and BALC and BALF were retained for analysis of parameters indicative of inflammation, injury, and immune alteration as described for the time course study. The left lung was clamped during lavage and subsequently excised, homogenized, and cultured to evaluate bacterial clearance from the lung.

BAL. BAL was performed at each time point after exposure as previously described [539]. The acellular first fraction of fluid was retained for analysis of $L D H$, cytokines and chemokines. The BALC were counted, differentiated, and assess for oxidative potential as described previously.

BALF Analysis: LDH activity in the BALF of all treatment groups was measured spectrophotometrically at each time point after exposure to evaluate cytotoxicity as a measure of lung injury as previously described [539]. Cytokines and chemokines involved in inflammatory and immune responses were measured in the BALF at each time-point after exposure using commercially-available enzyme-linked immunosorbent assay (ELISA) kits or by multiplex array. The following cytokines and chemokines were quantified by ELISA: monocyte chemotactic protein (MCP)-1, macrophage inflammatory protein (MIP)-2 (Novex, Life Technologies; Grand Island, New York); and osteopontin (OPN, Enzo Life Sciences; Farmingdale, New York). Additionally, two matrix metalloproteinases (MMP), MMP-2 and MMP-9, and tissue inhibitor of metalloproteinases (TIMP)-1 were quantified by ELISA (R\&D Systems) as indices of tissue

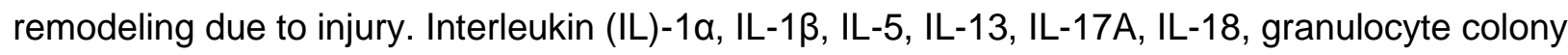
stimulating factor (G-CSF), granulocyte and macrophage colony stimulating factor (GM-CSF), eotaxin, fractalkine, leptin, MIP-1a, epidermal growth factor (EGF), IFN-y inducible protein (IP)10 , growth-regulated oncogene/keratinocyte chemoattractant (GRO/KC), vascular endothelial growth factor (VEGF), tumor necrosis factor- $\alpha$ (TNF- $\alpha$ ), transforming growth factor- $\beta 2$ (TGF- $\beta 2$ ), 
IL-2, IL-4, IL-6, IL-10, IL-12p70, interferon (IFN)-y, lipopolysaccharide (LPS)-induced CXC chemokine (LIX) and regulated on activation, normal T-cell expressed and secreted (RANTES) were assessed by multiplex array (Eve Technologies; Alberta, CA).

BALC and Mediastinal Lymph Node (MLN) Cell Differentiation and Phenotyping. Total BALC collected from rats were counted using a Coulter Multisizer II (Coulter Electronics; Hialeah, FL), followed by differentiation into alveolar macrophages (AMs) and neutrophils as previously described [539]. Flow cytometry was performed to enumerate and phenotype BALC. The total number of lymphocytes, B cells $(\mathrm{CD} 45 \mathrm{R})$, and $\mathrm{T}$ cells $\left(\mathrm{CD}^{+}\right)$, as well as $\mathrm{T}$ cell subsets $\left(\mathrm{CD}^{+} / \mathrm{CD} 8^{+}\right)$cells were quantified.

Chemiluminescence (CL). To measure the production of reactive oxidant species by BALC, $C L$ was measured according to the method of Antonini et al. [507] as previously described [539]. Briefly, extracellular oxidant production was measured as light emitted as a reaction of oxidants with luminol following stimulation of BAL AMs by zymosan or total BAL phagocytes (AMs and neutrophils) by phorbal myristate acetate (PMA).

Histopathology. The left lungs of rats were fixed with 10\% neutral buffered formalin by airway pressure fixation under $30 \mathrm{~cm}$-water pressure to total lung capacity for $15 \mathrm{~min}$. Lung volumes were assessed by weight of water displacement. The left lungs were embedded in paraffin, sectioned onto slides, and stained with hematoxylin and eosin (H\&E) or trichrome. An $n=5$ stained slides per treatment group per time-point per study block were assessed; blocks were designed with paired controls resulting in $n=10$ for saline and SIL groups from each of the blocks when combined. Stained slides were analyzed for indications of inflammation, injury, and fibrosis by a certified veterinary pathologist at Charles River Laboratories (Wilmington, MA) who was blinded to the treatment groups. Indices of pathology were scored on scale of $0-5$, where $0=$ no observed effect, $1=$ minimal response, $2=$ mild response, $3=$ moderate response, $4=$ marked response, and $5=$ severe response.

Determination of Particle Clearance - Clearance of particles from the lungs was evaluated both qualitatively by examining representative images of particle loaded phagocytes from cytospins using light field microscopy on an Olympus BX63 using CellSens Dimensions Software version 1.15 (Olympus, Tokyo, Japan) and quantitatively by morphometrical analysis of tissue section. Tissue sections from left lung were deparaffinized and stained lightly with Sirius Red. Slides were immersed in $0.1 \%$ picrosirius solution (100 mg of Sirius Red F3BA in $100 \mathrm{ml}$ of saturated aqueous picric acid) for $2 \mathrm{~h}$ followed by washing for $1 \mathrm{~min}$ in $0.01 \mathrm{~N} \mathrm{HCl}$. 
Slides were then counterstained with hematoxylin for $2 \mathrm{~min}$, dehydrated, and mounted with a coverslip for imaging. Quantitative morphometric methods were used to measure the volume (\% of the alveolar wall) and enumerate particles present by standard morphometric analyses (Underwood 1970; Mercer et al. 1994). This consisted of basic point counting. An eyepiece counting overlay consisting of 11 by 11 lines (121 total points for each throw of the overlay) was used with a 40X objective. A grid pattern for throws of the counting overlay was used in order to ensure a uniform sampling of the section, which did not overweigh interior points. An $n=5$ slides was evaluated for each group at time-points of 1 day, 1 week, 1 month, and 3 months following exposure. Briefly, clearance was calculated by finding the total volume of particle, normalizing data to 1 at day 1 and deriving a natural log curve from latter time-points. Tissue stained lightly with Sirius red was examined by dark-field microscopy on an Olympus BX41 using CellSens Dimensions Software version 1.15 (Olympus, Tokyo, Japan) at a magnification of 20x. Representative images of total particle load were captured using darkfield microscopy at 1 day, 1 month, and 3 months to illustrate the morphometric findings.

Bacterial Clearance from the Lung. To measure the clearance of bacteria from the lung, left lungs were excised and placed in $10 \mathrm{ml}$ of sterile deionized water. Lungs were homogenized using a Polytron $\circledast$ PT-2100 homogenizer with PT-DA 2112/EC aggregate attachment (Kinematica; Bohemia, NY). Homogenates were diluted and cultured on prepared Brain Heart Infusion Agar plates (Becton, Dickinson and Company). Cultures were incubated at $37^{\circ} \mathrm{C}$ for 24 h. Cfu were counted and multiplied according to culture dilution factors.

Statistical Analyses. Results for all BAL parameters and bacterial clearance were expressed as mean \pm standard error. For BAL proteins that were below the limit of detection, 1/2 of the lowest limit of detection was used for statistical analysis of values. Lymphocyte phenotype derived from flow cytometry was expressed as fold change from control \pm standard error calculated as $\frac{\Delta \bar{x}}{\text { control } \bar{x}}$ and a one-way analysis of variance (ANOVA) was performed between all treatments groups at each time point. Significant differences among groups were determined using the Student-Newman-Keuls post-hoc test. Data were analyzed using SigmaPlot for Windows Version 12.5 (Systat Software, Inc, Ekrath, Germany). For all analyses, significance was set at $p<0.05$. Because data from histopathology studies are inherently categorical, nonparametric analysis of variance was assessed using SAS/STAT software, Version 9.1, of the SAS System for Windows statistical programs (SAS Institute, Inc.; Cary, NC). In this instance, groups were compared using the Wilcoxon rank sum test. 


\section{Results}

Particle Characterization. One $\mathrm{ml}$ aliquots of $233 \mu \mathrm{g}$ dose DPM, $233 \mu \mathrm{g}$ dose CB, $233 \mu \mathrm{g}$ dose of SIL, $233 \mu \mathrm{g}$ DPM in combination with SIL $233 \mu \mathrm{g}$ (DPM/SIL), and $233 \mu \mathrm{g}$ CB in combination with SIL $233 \mu \mathrm{g}$ (CB/SIL) in PBS solution, were dried on carbon filters and examined using FESEM in order to characterize size of the primary particles and their degree of agglomeration. The primary particles of SIL were approximately $2 \mu \mathrm{m}$ in size and were evenly distributed in suspension (Figure 1A, 1B). DPM and CB were found to agglomerate in the vehicle to a size of approximately 1-2 $\mu \mathrm{m}$ in diameter. The primary particle size of DPM was found to be smaller in size than SIL, <200 nm (Figure 1A). The form of CB selected (Printex 90) had a primary particle size of $\sim 20 \mathrm{~nm}$ (Figure 1B). This form of CB was selected over fine or coarse mode CB as their primary particles were larger than the DPM agglomerates and the surface area and particle number per mass did not correlate well to the DPM represented. The FESEM images show similar agglomerates and primary particle size of the DPM and CB. Additionally, the association between DPM and SIL or CB and SIL in solution was minimal.

Time-Course Study. Lung injury was evaluated by examining LDH in the BALF collected from rats at 1 day, 1 week, 1 month, 2 months, and 3 months post-exposure to particles (Figure 2). A significant increase in LDH activity resulting from exposure to DPM alone occurred at 1 day and 1 week post-exposure, and at 1 day post-exposure for CB-exposed animals. SIL caused a significant increase beginning at 1 week post-exposure that persisted throughout the time course. LDH activity was significantly increased in both the DPM/SIL and CB/SIL groups at all time-points when compared to control, DPM, and CB. In the DPM/SIL group the increase was greater than all other groups at 1,2, and 3 months post-exposure, whereas the CB/SIL and SIL group were comparable to each other at these time-points.

Cell differentials were performed on BALC to further assess inflammation (Figure 3A\&B). DPM alone caused an increase in AMs in the lungs at 1 day post-exposure when compared to controls. CB alone did not increase AM number at any time point. SIL alone caused increased AM influx into the lungs at the later time-points (1 month and following). Increased AMs following exposure to CB/SIL did not differ greatly from that of SIL at the later time-points. However, DPM/SIL caused a significantly greater increase in AMs when compared to all groups at 1,2, and 3 months post-exposure. DPM exposure alone did not cause an increase in BAL neutrophils at any time-point. CB exposure alone caused an increase in neutrophil recruitment at day 1 post-exposure only. SIL, DPM/SIL and CB/SIL caused a significant increase in 
neutrophils at all time-points following exposure. At the later timepoints, SIL and CB/SIL did not differ from each other; however, exposure to DPM/SIL caused a significant increase when compared to all groups.

Oxidant production by BALC was evaluated ex vivo (Figure 3C\&D). DPM alone did not cause a significant increase in oxidant production by total phagocytes (Figure 3C) nor AMs (Figure 3D) as measured by CL at any of the time points. CB and SIL alone caused increased AM oxidant production at 1 day following exposure compared to control and DPM, although there was a trend for an increase in the SIL group at the later time points when compared to control. The CB/SIL group caused a significant increase in AM oxidant production up to 1 month postexposure when compared to control and at 1 week and 1 month when compared to all individual particle groups. Although not significantly different from control, the increase persisted at 2 and 3 months post-exposure and was comparable to that of SIL. Interestingly, exposure to DPM combined with SIL resulted in significantly increased and synergistic production of oxidants beyond that of exposure to any single particle alone at all time-points post-exposure for total phagocytes and at all time-points post-exposure with the exception of 1 week for AM oxidant production.

BAL and MLN lymphocytes were phenotyped by flow cytometry (Table 1\&2). Exposure to DPM alone caused only mild increases in lymphocyte populations in BAL with the exception of a trend for increased T cells observed at 1 month becoming significant at 2 months. DPM caused increased B cells at 1 week and 1 month post-exposure (Table 1). However, at the early time points DPM alone did increase all MLN lymphocyte proliferation (Table 2), and though these populations remained elevated compared to control throughout the time-course, this result was not significant after 1 week. CB alone did not cause any increases in lymphocyte populations when compared to control. In addition to phagocytic influx described in Figure 3, exposure to SIL alone caused significantly increased lymphocyte influx into the lung (Table 1) and increased proliferation in the MLN (Table 2) as early as 1 week following exposure. When SIL was combined with DPM, proliferation of MLN lymphocytes were observed as early as 1 day postexposure (Table 2). Influx of lymphocytes was primarily attributed T cell population subsets, specifically $\mathrm{CD}^{+}{ }^{+}$cells, though all subsets were increased for the DPM/SIL co-exposure group. CB in combination with SIL did increase the influx of lymphocytes in the BAL between 1 week and 3 months for $T$ lymphocytes, primarily due to increased $C D 4^{+}$cells. Interestingly, there were no significant increases in lymphocytes in the MLN following CB/SIL exposure. 
Protein mediators were evaluated in BALF as a measure of inflammation and immune response (Table 3-5). CB caused some transient changes, particularly for inflammatory proteins including MCP-1, MIP-1 $\alpha$, and TNF- $\alpha$, at the earliest time-point when compared to control (Table 3). There is a trend for increased MIP-2 by CB but this is only significant when compared with DPM groups, which demonstrated decreased expression of this chemokine compared to control. IP10 expression was also increased by $\mathrm{CB}$ at 1 day following exposure (Table 4). Similar to inflammatory proteins, CB increased expression of tissue remodeling proteins in the BALF at the earliest time-point (Table 5). DPM alone elicited only an increase in MIP-1 $\alpha$, TNF- $\alpha$, IL-18, IP-10, and TGF $\beta-2$. However, these changes were not consistent throughout the time course (Table 3-5). Conversely, DPM exhibited a trend for reduced expression of some proteins including MIP-2, IL-6, IL-10, IL-12p70, and OPN, throughout the time course (Tables 3-5), and IL-2, IL-4, and IFN-y at the latest time-points (Table 4). SIL alone caused increases in proteins associated with inflammation including LIX, MCP-1, MIP-1 $\alpha$, MIP-2, and TNF- $\alpha$, at most timepoints compared with the control and/or groups exposed to DPM or CB only. SIL alone also caused an increase in IL-18, a protein associated with upregulation of the NLRP3/NALP3 inflammasome (Table 3). Further, proteins associated with tissue remodeling, OPN and MMP-9, which have implications in the development of fibrotic changes in the lung, were increased by treatment with SIL particularly at time points after 1 week (Table 5). DPM, when in combination with SIL, increased expression of MIP-1 $\alpha$, IL-18, and TNF- $\alpha$ at some time-points (Table 3), and tissue remodeling proteins including MMP-9, TGF $\beta 2$, and TIMP-1 throughout the time course, as well as, OPN at time points as early as 1 month (Table 5). CB in combination with SIL showed effects very similar to SIL for most inflammatory proteins including LIX, MCP-1, MIP1- $\alpha$, MIP-2, IL-18, and TNF- $\alpha$ (Table 3), though at some early time-points CB/SIL increased expression of MCP-1 and MIP-2 over that of SIL alone. Similarly to CB alone, CB combined with SIL increased expression of IP-10, particularly at earlier time-points. This was also observed for DPM/SIL at 1 week and 1 month. CB/SIL also enhanced the expression of tissue remodeling proteins MMP-9, OPN, and TGF 32 throughout the time course, similar to that of DPM/SIL suggesting that these proteins may be most responsive to overall load of particles. Lastly, in the co-exposure with SIL, DPM exhibited independent action, reducing the expression of LIX, MIP2, IL-2, IL-4, and IL-12p70 at some time-points compared to SIL (Table 3\&4). Interestingly, this was not the case for CB/SIL, suggesting specific properties of DPM may be responsible for this effect. There were no significant changes in the following cytokines in any group: IL-1 $\alpha, I L-1 \beta$, IL-5, IL-13, IL-17A, EGF, eotaxin, fractalkine, GRO/KC, G-CSF, GM-CSF, leptin, IFN- $\mathrm{y}, \mathrm{MMP}-2$, and VEGF (data not shown). 
H\&E and trichrome-stained tissue sections were evaluated by a board-certified veterinarian pathologist (Table 6). DPM caused only mild influx of AMs, as a measure of inflammation; however, this did not differ significantly from control. SIL alone and in combination with DPM or $\mathrm{CB}$, significantly increased influx of AMs at all time-points following exposure. Alveolar epithelial hyperplasia $(\mathrm{AEH})$ was also observed following co-exposures with both particles beginning at 1 month and persisting up to 3 months. SIL alone also increased AEH at 2 and 3 months postexposure. In general, the degree of significance was greater for the DPM/SIL group when compared to control versus the SIL and CB/SIL group when compared to control. Beginning at 1 month following exposure to particles, minimal to mild septal fibrosis was observed in groups that contained SIL. At 1 month, the incidence of fibrosis was greatest in the DPM/SIL coexposure group ( $60 \%$ of animals), whereas $20 \%$ of animals had fibrosis in the SIL and CB/SIL groups. At 2 months post-exposure, the DPM/SIL and CB/SIL group had a similar degree of fibrosis, which was greater than that of SIL alone. By 3 months post-exposure, the degree of fibrosis in all groups containing SIL were similar to each other, with a trend toward the coexposures having a slightly greater incidence. Mild hyperplasia of the bronchiolar/alveolar epithelium was observed near the terminal bronchioles as early at 1 month following exposure to groups containing SIL and was found to be associated with the presence of macrophages loaded with high particle content, suggesting a correlation between tissue damage and the presence of particles. Overall, most histopathological changes were attributable to exposure to $233 \mu \mathrm{g}$ of SIL; however, the presence of DPM or CB increased the severity and/or incidence of some of these changes in the co-exposure groups, suggesting particle load as an additional factor in toxicity. DPM increased the onset of fibrosis, but not the degree of fibrosis, which was attributable primarily to particle load.

Lung particle burden and clearance were evaluated both qualitatively in tissue and macrophages (Figures 5-10) and quantitatively in tissue by morphometric analysis (Figure 4A). Between day 1 and week 1 approximately $2 \%$ of DPM was cleared with an increase in the rate of clearance following the first week (Figure 4A). By 1 month, 55\% of DPM had been cleared; after 1 month there was a steady decline in particle burden with only $15 \%$ overall DPM remaining at 3 months post-exposure. CB was cleared at a faster rate initially, with only $60 \%$ of particle remaining at 1 week following exposure, and this is evident by the macrophage burden of particle observed at 1 day as compared with that of DPM (Figure 5). By 1 month, the clearance of CB had slowed to nearly that of DPM with $16 \%$ of particles remaining at 3 months post-exposure. Initial clearance of SIL between the time-points of 1 day and 1 week was similar to $\mathrm{CB}$, and faster than DPM, with nearly $30 \%$ of particle cleared; the rate of clearance slowed 
after 1 week and by 1 month only 45\% SIL particle had been cleared (Figure 4A). Following this, there was only approximately an additional 10\% clearance of particle by 3 months, suggesting that the rate of clearance for SIL slows significantly after 1 month. Clearance in the co-exposure groups was significantly slower than any individual particle at time-points following 1 week as expected by increase in overall particle load as can be seen in figures 7-10. By 1 week, between $2-3 \%$ of particle was cleared for the CB/SIL or DPM/SIL groups and the rate of clearance remains very slow compared with individual particles up to 1 month following exposure where only measurable clearance was observed for the CB/SIL group since 1 week following exposure (Figure 4A). By 3 months post-exposure approximately $35 \%$ of particle burden had been cleared.

Infection Study. Respiratory susceptibility to a bacterial pathogen was determined following particle exposure in an infectivity study assessing the clearance of LM from the lung over a 14day time course, 1 week following exposure to particles. Despite lung inflammation and phagocytic cell activity observed in the absence of infection in co-exposed groups (Figure 3), overall clearance of the pathogen was not significantly altered for most time-points (Figure 11). Clearance in the CB/SIL co-exposure was enhanced at 1 day compared with control and CB alone. At 3 days following infection the slowest degree of bacterial clearance was associated with the DPM group; whereas, all groups that contained SIL were comparable to control or cleared the bacteria faster than DPM alone. However, by 5 days post-exposure there were no differences in bacterial clearance among groups. Neutrophil influx into the lung was significantly increased for SIL-containing groups at day 1 post-infection (Figure 12B). By day 3 postinfection, the influx of cells in SIL- exposed groups was similar to control with the exception of neutrophils, which were enhanced beyond that of CB or DPM alone groups at day 14. Influx of lymphocytes into the BAL at day 14 was also significantly increased in co-exposed groups (Figure 13A). Similarly, proliferation of lymphocytes in the MLN was elevated in groups that contained SIL as early as 1-day post-infection, and significantly elevated again by day 14 in the co-exposed groups (Figure 13B). BAL and MLN lymphocytes were phenotyped as in the timecourse study to evaluate lymphocyte populations. Neither DPM nor CB exposure caused notable lymphocytic influx into the lung when compared to infected controls, while combined exposure to particles caused significant increases in total lymphocyte influx at day 14 compared with other groups, attributed primarily to an increase in T lymphocytes (Table 7). In the MLN, combined exposure followed by infection caused increased lymphocytes at day 1 compared with all other non-SIL-containing groups. For co-exposure groups, proliferation of all MLN cells was greater than SIL for all subsets (Table 8). At 14 days post-infection T cell-subsets were 
increased in both co-exposed groups, attributed mainly to an increase in CD4 ${ }^{+}$and $\mathrm{CD} 8^{+} \mathrm{T}$ lymphocytes. Overall, the results suggest that DPM may have impaired the innate response to infection to a degree; whereas, the presence of SIL may have enhanced the response at the early time-points to a degree. By day 14 , the infection had resolved and the observed increased inflammation and immune cell proliferation may be largely attributable to particle effect.

\section{Discussion}

Our previous study had shown that low- to no-toxicity doses of DPM in combination with SIL caused increased inflammation and lung injury when compared to SIL alone [539]. The goal of the present study was to determine if those effects were particle-specific or if the effects were due to an increased particle load as a result of the co-exposure. It is well-established that overall particle load contributes to the development of disease in the lung. Particles with known low cytotoxicity or particles not otherwise classified/regulated (PNOC/R) due to low toxicity have been shown in animals to contribute to inflammation, fibrosis, and tumor development if the number/mass of particles administered exceeds the capabilities of alveolar macrophages to adequately clear them [542-546]. In order to address these questions, the current study was designed examining an equivalent dose of DPM particles as SIL alone, DPM in combination with SIL, and CB at an equivalent dose to DPM alone and in combination with SIL as a reference particle for load control.

In the present study, DPM and CB alone produced inflammation and lung injury only at early time-points post-exposure and resolved by 1 week to 1 month. SIL alone increased parameters of inflammation and lung injury throughout the time course. In several instances, the co-exposures to DPM or CB and SIL further enhanced these effects, which may be attributed to particle-specific effect or to load effects depending on the parameter. In general, DPM/SIL had a greater effect than $\mathrm{CB} / \mathrm{SIL}$ on parameters including lung injury (cytotoxicity), cellular influx into the lung, and ex vivo oxidant production by phagocytes in the lung, indicating a particle-specific effect relating to a constituent other than the carbon core of DPM; whereas particle load and the presence of SIL appeared to affect tissue remodeling and fibrosis to a greater degree, although DPM co-exposed with SIL did increase the incidence of pathology at 1 month following exposure when compared to SIL or CB/SIL groups .

In the case of cytotoxicity, while both combined exposures caused significant toxicity compared with control at all time-points following exposure, DPM combined with SIL increased cytotoxicity examined at all time-points following 1 week (Figure 2). Though CB in combination 
with SIL also increased cytotoxicity after 1 month, this effect was similar to the level caused by SIL alone suggesting that for the DPM/SIL combo group, DPM characteristics and not simply the carbon core of DPM enhanced cytotoxicity. Further, this suggests that it is not simply the overall load of nearly $500 \mu \mathrm{g}$ of particles contributing to cell injury. DPM also had particlespecific effects on the influx of phagocytes into the lung and their oxidant producing activities (Figure 3). Though DPM alone had little effect on the influx of phagocytes into the lung, when combined with SIL, the presence of macrophages and neutrophils were significantly increased compared to all groups and therefore exacerbated the inflammation observed for SIL alone or $\mathrm{CB} / \mathrm{SIL}$. These effects were observed beginning at one month following exposure for both macrophages and neutrophils. In the case of oxidants produced by either total phagocytes or macrophages alone, DPM when combined with SIL, synergistically enhanced this effect (Figure 3 C\&D). The same effect was not observed in the CB/SIL co-exposure. Though CB/SIL enhanced oxidant production by macrophages alone above that of SIL, the effect was diminished after 1 month. Because the rate of particle clearance in the combined exposures is similar after 1 month, it is unlikely that these effects are driven entirely by particle burden. Taken together, these results suggest that DPM in combination with SIL not only enhanced the influx of phagocytic cells into the lung but also increased their function with regard to the production of oxidants. Moreover, these results suggest that though particle load may play a role in these findings, DPM specific properties also contribute to the overall effect, particularly in the case of total oxidant burden that reached as high as 100 -fold change from control at some time-points for total phagocytes (Figure 3C).

With regard to the influx of lymphocytes into the lung, CB alone had no effect (Table 1). DPM caused slightly enhanced influx of all immune cells examined when compared to control, particularly at the earlier time-points. SIL resulted in significantly increased influx of cells at most time-points following exposure, attributable primarily to T lymphocytes. When DPM was combined with SIL these results were further exacerbated. Though combination of CB with SIL did enhance the influx of lymphocytes at some time-points this effect was significantly greater for DPM/SIL and therefore suggests that while particle load may contribute to stimulation of lymphocyte influx into the lung, DPM-specific properties further enhance this response. Similar findings were observed for the proliferation of lymphocytes in the MLN (Table 2). Interestingly, there was a trend of increased MLN proliferation for DPM in a co-exposure with SIL as compared with control that was not observed for the CB/SIL co-exposure. This finding would suggest that although SIL plays a significant role in many facets of pulmonary toxicity relating to 
this co-exposure, DPM is also contributing significantly, particularly in the context of immune alteration.

Similarly, SIL exposure resulted in increases in several inflammatory, immunoregulatory, and tissue remodeling proteins in the lungs (Tables 3-5). Although both DPM/SIL and CB/SIL led to equal or enhanced expression of some of these proteins including: MCP-1, MIP-1a, IP-10, and MMP-9. DPM reduced the expression of others including: LIX, MIP-2, IL-2, IL-4, and IL-6 in the BALF when in combination with SIL. DPM appeared to be exhibiting independent action on the expression of these proteins possibly stemming from the suppression of macrophage function. Monocytes or macrophages secrete these proteins in order to recruit additional phagocytes to the site of acute inflammation, as well as to cause autocrine and paracrine activation of cells, and to differentiate lymphocytes (Table 3-5). Furthermore, these results were not reflected in the CB/SIL co-exposure suggesting that, despite increased cellular recruitment to the lung, potential suppression of macrophage function may be occurring and may be due to specific properties related to DPM and not simply an effect of overall particle load. These findings are in agreement with conclusions made by Yin et al. that organic fractions of DPM result in the reduced expression of cytokines from AMs [316, 535]. This is effect is also evident in the innate response to LM infection whereby pulmonary clearance of the bacteria was slowest in the DPM only group at day 3.

Pathological tissue analysis showed that either DPM or CB alone had little influence on the histological changes evaluated, with the exception of the incidence of AM influx into the lung (Table 6), and the presence of dark granules noted within macrophages throughout the time course, representative of particles that had been phagocytosed. Further, brown granules observed by the pathologist were scored as more severe in groups exposed to combinations of particles when compared to single particle groups, particularly at later time-points, which agrees with the morphometrical finding of reduced clearance of particles in these groups (data not shown). Tissue from animals exposed to SIL alone or groups containing SIL were characterized by enhanced inflammation, alveolar $(\mathrm{AEH})$ and bronchial epithelial hyperplasia $(\mathrm{BEH})$, and alveolar septal thickening or fibrosis. Though these parameters were enhanced over that of SIL at times in the co-exposure groups, as in the case of $\mathrm{BEH}$ and $\mathrm{AEH}$ at 1 month post-exposure, these effects were correlated better with overall particle load at the later time-points, as there were no observed differences between the two co-exposure groups. Furthermore, these findings are supported by the observed increases in tissue remodeling proteins expressed in the BALF at these time-points. Increases in MMP-9 and OPN were observed throughout the time 
course with significant differences observed at 1 month post-exposure in the combined groups compared with single dose groups. These findings correlate with the earlier incidence of hyperplasia developing for these groups compared with SIL alone. Also at 1 month postexposure, there are particle specific effects of DPM evident in the incidence of fibrosis and AEH that may have related to increases in the expression of tissue remodeling proteins TGF $\beta$ and TIMP-1 in the DPM/SIL groups at this time-point. This finding was also apparent when qualitatively assessing tissue by dark field microscopy (Figure 7). Interestingly, particle clearance rate for the CB/SIL group was slightly increased compared with the DPM/SIL group between the time-points of 1 week and 1 month, which may also account for the increase in injury in the DPM/SIL group observed at this time-point (Figure 10).

Susceptibility to an infection following particulate exposure was also examined to further assess alterations in immune function following particle exposure. Neutrophilic influx, and proliferation of lymphocytes in the MLN (Figure 11, Table 8) were increased at 1 day following infection for all groups exposed to SIL. Differences between SIL exposure groups were not significant with the exception of lymphocyte proliferation in MLN at day 1 following infection in the CB/SIL group. This result correlates to increased clearance of bacteria from the lung at 1 day following infection observed for this group, although this result was only significant when compared with control and CB exposures. Clearance of bacteria was significantly enhanced for all groups except CB when compared to the DPM only group at day 3 following infection, suggesting further that DPM may lead to a degree of inhibition in the innate immune response early after infection. This effect is similar to other studies examining diesel exposure and infection. In a study examining inhalation exposure to total DE, Harrod et al. determined that exposure resulted in reduced clearance of Pseudomonas aeruginosa infection [313]. Similarly, Yin et al. demonstrated reduced clearance of bacteria from the lung of rats following DPM exposure, citing reduced phagocytic function and reduction in cytokine expression by macrophages as possible DPM-related effects resulting in increased susceptibility [316]. In a follow-up study, Yin et al. demonstrated increased bacterial burden at day 3 following coexposure of rats to DPM and LM. However, similar to the present study, despite reduced expression of cytokines by macrophages, rats were able to ultimately clear infection suggesting that the adaptive cell-mediated response was still sufficiently initiated despite the reduction in response of the innate immune system [317].

Our previous study examining a lower dose of DPM $(50 \mu \mathrm{g})$ indicated that DPM increased parameters of toxicity such as inflammation, oxidant production, and the onset of 
initiation of fibrosis in the co-exposure compared to SIL, particularly at 1 month following exposure. Though these effects were still comparable to SIL at 3 months post-exposure, they no longer exceeded that of SIL. Therefore, the present study examined whether the exacerbated effects of the combined exposure were no longer apparent compared to silica at 3 months time due to clearance of DPM or if the progression of effects due to SIL alone or particle load were the primary factor in toxicity. This study showed that combination of DPM and SIL at equivalent doses resulted in not only enhanced inflammation and oxidant production as previously observed, but increases in cytotoxicity as well. DPM also increased the incidence of fibrosis at 1 month post-exposure, similar to the previous study; however severity of fibrosis was similar between SIL and co-exposure groups. Further, by the later time-points, the incidence and severity were more similar in the co-exposure groups. This is in combination with the slower particle clearance rate of the co-exposure groups suggests that tissue remodeling initiated by SIL exposure and total particle load were the primary factors in progression of fibrosis (Table 6). Although SIL is the more toxic particle in the study as compared to DPM alone, when given at a dose equal to SIL, effects of DPM co-exposed with SIL were still apparent at 3 months following exposure, particularly for cytotoxicity, inflammation, and the production of oxidants. This was not the case for the $\mathrm{CB} / \mathrm{SIL}$ exposure at equivalent loads, suggesting these effects were particle specific for both the previous study, as well as this study.

Overall, this study indicated that both load and particle type, SIL and DPM, contribute to toxicity in a co-exposure scenario depending on the effect. The toxicological properties of crystalline silica and DPM are well-established individually. Both acute exposures to large doses of particles and chronic low-level exposure to silica are known to contribute to the development of fibrotic lung diseases related to oxidant-induced lung injury [4, 487, 490, 515, 516]. DPM is also known to induce inflammation resulting in oxidative stress [467, 473-476]. Although DPM is not typically associated with the restrictive fibrotic disease outcomes that are associated with silica exposure at doses below overload, these particles are known to result in obstructive lung diseases such as chronic obstructive pulmonary disease and emphysema, as well as cancer $[411,415,517,518]$. While specific mechanisms governing the progression of these diseases have been elucidated, many remain unknown. Delineating the mechanisms of action in a coexposure is further complicated by potential synergistic or antagonist actions of the materials. This study, however does show that SIL-induced inflammation and oxidant production are further enhanced by particle specific effects of DPM, but that particle load may also be a significant factor particularly at the later time points where clearance is slower in the co-exposed groups. 
It should be noted that the particles used for this study and Farris et al. (2017) were selected as surrogates for measuring toxicity of particles encountered in the workplace, which may be more complex in chemical nature and reactivity. Factors contributing to the workplace exposures would include freshly fractured silica, which is more reactive than the aged silica in this study. In addition, NIST SRM 2975 only represents the particulate component of diesel exhaust (DE). Whole DE, depending on the engine type, contains higher fractions of hydrocarbons, as well as metals, and these components may contribute to increased toxicity as compared to that observed in this study. These factors are important considerations in the interpretation of the data as it pertains to exposure in the workplace.

\section{Conclusion}

In the present study, DPM or CB alone caused a transient inflammation and lung injury, whereas SIL alone caused an increase in inflammation characterized by cellular influx into the lung, increased inflammatory mediator production in the lung, and alterations in lung histology. In general, co-exposure to DPM or CB and SIL followed a similar pattern as SIL alone, and, in some instances, the effects were increased relative to SIL alone suggesting particle-specific effects or effects due to load. SIL and particle load appear better correlated with tissue remodeling (fibrosis and tissue remodeling proteins in the lung) and some inflammatory proteins in the lung, including MCP-1, MIP-1a, and MIP-2. DPM combined with SIL increased production of oxidants by phagocytes compared with all other groups at all time-points post exposure. In addition, DPM/SIL co-exposure enhanced AMs and neutrophil influx into the lung, lung injury (LDH), the influx of lymphocytes in the lung, the proliferation of lymphocytes in the MLN, and the incidence of epithelial hyperplasia in lung tissue at later time-points compared to exposure to SIL alone. Although CB/SIL co-exposure increased lung injury and cellular influx into the lung, as well as epithelial hyperplasia, the increase was comparable to that of SIL alone. The data taken together show that DPM exposure at doses that elicit only minimal pulmonary toxicity has the capacity to significantly alter SIL-induced pulmonary effects, primarily cytotoxicity, inflammation, oxidant production, lymphocytic proliferation, as well as exhibit independent action on the expression of immune protein mediators of inflammation. Furthermore, comparison of these effects to a reference particle for size and particle load comparison revealed that many of these findings relate directly to unique characteristics of DPM rather than being related only to dose administered. 
Table 1: Lymphocyte Phenotype of BALC

\begin{tabular}{|c|c|c|c|c|c|c|}
\hline \multirow{6}{*}{ 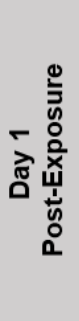 } & Exposure $(\mu \mathrm{g})$ & $\begin{array}{c}\text { Total } \\
\text { Lymphocytes }\end{array}$ & T Cells & CD4 $^{+}$T Cells & CD8 ${ }^{+}$T Cells & B Cells \\
\hline & DPM 233 & $1.60 \pm 0.47$ & $0.36 \pm 0.67$ & $0.52 \pm 0.81$ & $0.05 \pm 0.28$ & $0.03 \pm 0.32$ \\
\hline & CB 233 & $-0.27 \pm 0.10$ & $0.17 \pm 0.24$ & $0.50 \pm 0.28$ & $-0.12 \pm 0.31$ & $-0.22 \pm 0.25$ \\
\hline & SIL 233 & $0.72 \pm 0.50$ & $0.38 \pm 0.27$ & $0.62 \pm 0.31$ & $0.16 \pm 0.23$ & $0.21 \pm 0.22$ \\
\hline & DPM/SIL 233/233 & $1.00 \pm 0.26$ & $1.02 \pm 0.54$ & $1.27 \pm 0.54$ & $1.09 \pm 0.63$ & $0.63 \pm 0.26$ \\
\hline & CB/SIL 233/233 & $-0.21 \pm 0.21$ & $0.36 \pm 0.39$ & $0.68 \pm 0.48$ & $0.24 \pm 0.39$ & $-0.19 \pm 0.31$ \\
\hline \multirow{5}{*}{ 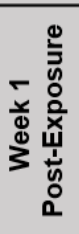 } & DPM 233 & $0.21 \pm 0.17$ & $1.25 \pm 0.52$ & $1.41 \pm 0.63$ & $1.25 \pm 0.76$ & $0.19 \pm 0.30$ \\
\hline & CB 233 & $-0.60 \pm 0.06$ & $-0.22 \pm 0.19$ & $-0.14 \pm 0.23$ & $-0.16 \pm 0.16$ & $-0.34 \pm 0.08$ \\
\hline & SIL 233 & $1.22 \pm 0.49^{d}$ & $1.47 \pm 0.56$ & $1.88 \pm 0.70$ & $1.44 \pm 0.61$ & $0.12 \pm 0.17$ \\
\hline & DPM/SIL 233/233 & $4.22 \pm 0.50^{\mathrm{e}}$ & $10.40 \pm 1.51^{e}$ & $11.61 \pm 1.69^{e}$ & $10.53 \pm 1.71^{e}$ & $1.09 \pm 0.31^{\mathrm{e}}$ \\
\hline & CB/SIL 233/233 & $0.87 \pm 0.19$ & $5.11 \pm 0.90^{c}$ & $6.60 \pm 1.13^{c}$ & $3.51 \pm 0.79^{d}$ & $0.43 \pm 0.15$ \\
\hline \multirow{5}{*}{ 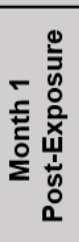 } & DPM 233 & $1.01 \pm 0.34$ & $1.74 \pm 0.65$ & $1.81 \pm 0.58$ & $0.65 \pm 0.79$ & $3.50 \pm 1.24^{b}$ \\
\hline & CB 233 & $-0.73 \pm 0.03$ & $-0.60 \pm 0.07$ & $-0.56 \pm 0.08$ & $-0.70 \pm 0.07$ & $-0.19 \pm 0.28$ \\
\hline & SIL 233 & $1.37 \pm 0.57^{g}$ & $1.89 \pm 0.71$ & $2.85 \pm 0.98$ & $0.59 \pm 0.34$ & $2.00 \pm 0.89$ \\
\hline & DPM/SIL 233/233 & $4.44 \pm 0.81^{e}$ & $10.38 \pm 1.32^{\mathrm{e}}$ & $14.42 \pm 1.93^{e}$ & $3.75 \pm 0.74$ & $5.05 \pm 1.24$ \\
\hline & CB/SIL 233/233 & $0.24 \pm 0.52$ & $4.49 \pm 2.96$ & $5.93 \pm 3.52^{\mathrm{d}}$ & $4.97 \pm 4.48$ & $0.43 \pm 0.57$ \\
\hline \multirow{5}{*}{ 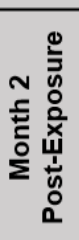 } & DPM 233 & $1.27 \pm 0.48^{g}$ & $2.60 \pm 1.11^{d}$ & $2.41 \pm 0.94$ & $3.22 \pm 2.51$ & $5.26 \pm 1.65^{\mathrm{e}}$ \\
\hline & CB 233 & $-0.57 \pm 0.07$ & $-0.71 \pm 0.03$ & $-0.74 \pm 0.04$ & $-0.69 \pm 0.03$ & $-0.53 \pm 0.12$ \\
\hline & SIL 233 & $1.68 \pm 0.59^{j}$ & $2.27 \pm 0.69^{d}$ & $3.17 \pm 0.92^{d}$ & $0.99 \pm 0.37$ & $0.89 \pm 0.44$ \\
\hline & DPM/SIL 233/233 & $3.87 \pm 0.42^{e}$ & $8.03 \pm 1.33^{e}$ & $10.26 \pm 1.72^{e}$ & $7.01 \pm 1.81^{e}$ & $2.44 \pm 0.40^{\prime}$ \\
\hline & CB/SIL 233/233 & $0.33 \pm 0.11$ & $2.24 \pm 0.44^{d}$ & $3.11 \pm 0.60^{d}$ & $0.85 \pm 0.18$ & $-0.40 \pm 0.09$ \\
\hline \multirow{5}{*}{ 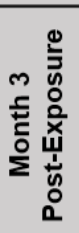 } & DPM 233 & $0.82 \pm 0.15$ & $0.88 \pm 0.22$ & $0.93 \pm 0.26$ & $0.37 \pm 0.50$ & $0.88 \pm 0.40$ \\
\hline & CB 233 & $-0.71 \pm 0.03$ & $-0.65 \pm 0.04$ & $-0.66 \pm 0.05$ & $-0.54 \pm 0.06$ & $-0.34 \pm 0.11$ \\
\hline & SIL 233 & $1.12 \pm 0.52^{j}$ & $2.95 \pm 0.78^{d}$ & $3.75 \pm 0.96^{d}$ & $4.61 \pm 1.30^{k}$ & $1.25 \pm 0.51^{\mathrm{j}}$ \\
\hline & DPM/SIL 233/233 & $3.43 \pm 0.59^{e}$ & $8.49 \pm 1.92^{e}$ & $11.02 \pm 2.52^{e}$ & $6.64 \pm 1.76^{k}$ & $2.01 \pm 0.44^{\mathrm{j}}$ \\
\hline & CB/SIL 233/233 & $-0.34 \pm 0.06$ & $1.29 \pm 0.29$ & $1.86 \pm 0.38$ & $1.39 \pm 0.36$ & $-0.26 \pm 0.14$ \\
\hline
\end{tabular}

All data are shown as mean fold change over control \pm standard error: ${ }^{\mathbf{b}}$ different from control only; ${ }^{\mathrm{c}}$ different from control and all single dose groups; ${ }^{\mathrm{d}}$ different from control and $\mathrm{CB}$; ${ }^{\mathrm{e}}$ different from all other groups; ${ }^{9}$ different from CB only; ${ }^{i}$ different from control and both $\mathrm{CB}$ groups; ' different from control, DPM, and both CB groups; and 'different from control, SIL, and both CB groups. Statistical significance is $p \leq 0.05$. 
Table 2: Lymphocyte Phenotype in the MLN

\begin{tabular}{|c|c|c|c|c|c|c|}
\hline \multirow{6}{*}{ 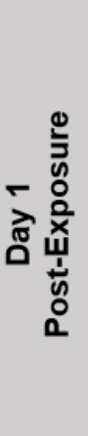 } & Exposure ( $\mu \mathrm{g})$ & \begin{tabular}{|c|} 
Total \\
Lymphocytes
\end{tabular} & T Cells & $\mathrm{CD}^{+}{ }^{\mathrm{T}}$ Cells & CD8 + T Cells & B Cells \\
\hline & DPM 233 & $1.62 \pm 0.33^{\prime}$ & $1.71 \pm 0.33^{\prime}$ & $1.63 \pm 0.33^{\prime}$ & $1.90 \pm 0.34^{\prime}$ & $1.45 \pm 0.36^{1}$ \\
\hline & CB 233 & $-0.50 \pm 0.10$ & $-0.43 \pm 0.11$ & $-0.43 \pm 0.11$ & $-0.42 \pm 0.12$ & $-0.51 \pm 0.09$ \\
\hline & SIL 233 & $0.32 \pm 0.35$ & $0.17 \pm 0.26$ & $0.07 \pm 0.25$ & $0.16 \pm 0.26$ & $-0.07 \pm 0.22$ \\
\hline & DPM/SIL 233/233 & $1.53 \pm 0.20^{1}$ & $1.58 \pm 0.18$ & $1.53 \pm 0.19^{1}$ & $1.66 \pm 0.271$ & $1.39 \pm 0.26^{\prime}$ \\
\hline & CB/SIL 233/233 & $-0.60 \pm 0.04$ & $-0.56 \pm 0.04$ & $-0.58 \pm 0.03$ & $-0.53 \pm 0.05$ & $-0.59 \pm 0.06$ \\
\hline \multirow{5}{*}{ 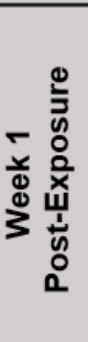 } & DPM 233 & $1.68 \pm 0.24$ & $1.67 \pm 0.35 \mathrm{j}$ & $1.79 \pm 0.37$ & $1.35 \pm 0.41^{j}$ & $1.29 \pm 0.33^{j}$ \\
\hline & CB 233 & $-0.44 \pm 0.09$ & $-0.39 \pm 0.10$ & $-0.36 \pm 0.10$ & $-0.50 \pm 0.08$ & $-0.46 \pm 0.08$ \\
\hline & SIL 233 & $0.86 \pm 0.419$ & $0.78 \pm 0.36^{g}$ & $0.82 \pm 0.36^{g}$ & $0.61 \pm 0.339$ & $0.59 \pm 0.289$ \\
\hline & DPM/SIL 233/233 & $2.16 \pm 0.31^{1}$ & $2.56 \pm 0.34$ & $2.70 \pm 0.36$ & $2.27 \pm 0.32^{\prime}$ & $1.63 \pm 0.34^{\prime}$ \\
\hline & CB/SIL 233/233 & $0.01 \pm 0.12$ & $0.14 \pm 0.15$ & $0.15 \pm 0.14$ & $0.06 \pm 0.17$ & $-0.04 \pm 0.09$ \\
\hline \multirow{5}{*}{ 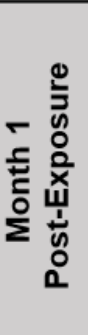 } & DPM 233 & $1.24 \pm 0.38$ & $1.28 \pm 0.41$ & $1.31 \pm 0.44$ & 0.37 & $0.97 \pm 0.32$ \\
\hline & CB 233 & $-0.74 \pm 0.03$ & $-0.72 \pm 0.04$ & $-0.71 \pm 0.04$ & $-0.76 \pm 0.03$ & $-0.74 \pm 0.03$ \\
\hline & SIL 233 & $1.51 \pm 0.77$ & $1.57 \pm 0.69 \mathrm{~g}$ & $1.50 \pm 0.63 \mathrm{~g}$ & $1.48 \pm 0.68$ & $1.03 \pm 0.52$ \\
\hline & DPM/SIL 233/233 & $6.07 \pm 0.96^{e}$ & $7.00 \pm 0.98^{e}$ & $6.82 \pm 0.96^{e}$ & $7.13 \pm 1.17^{e}$ & $5.38 \pm 0.95^{e}$ \\
\hline & CB/SIL 233/233 & $0.29 \pm 0.24$ & $0.61 \pm 0.34$ & $0.58 \pm 0.33$ & $0.63 \pm 0.37$ & $0.20 \pm 0.21$ \\
\hline \multirow{5}{*}{ 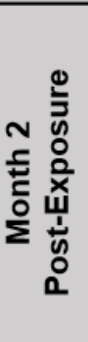 } & DPM 233 & $1.16 \pm 0.21$ & $1.17 \pm 0.20$ & $1.20 \pm 0.18$ & $1.11 \pm 0.30$ & $1.18 \pm 0.25$ \\
\hline & CB 233 & $-0.68 \pm 0.06$ & $-0.69 \pm 0.06$ & $-0.69 \pm 0.06$ & $-0.71 \pm 0.06$ & $-0.68 \pm 0.07$ \\
\hline & SIL 233 & $2.23 \pm 0.62^{\mathrm{d}}$ & $2.65 \pm 0.73^{d}$ & $2.30 \pm 0.65^{d}$ & $3.33 \pm 0.95^{d}$ & $2.14 \pm 0.61^{d}$ \\
\hline & DPM/SIL 233/233 & $5.14 \pm 1.03^{e}$ & $6.31 \pm 1.17^{e}$ & $5.57 \pm 1.03^{e}$ & $7.71 \pm 1.58^{e}$ & $4.24 \pm 0.90^{\mathrm{e}}$ \\
\hline & CB/SIL 233/233 & $1.51 \pm 0.44$ & $1.79 \pm 0.46$ & $1.59 \pm 0.39$ & $1.89 \pm 0.58$ & $1.18 \pm 0.38$ \\
\hline \multirow{5}{*}{ 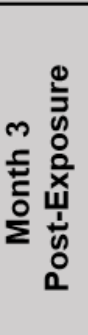 } & DPM 233 & $1.04 \pm 0.18$ & $1.31 \pm 0.25$ & $1.26 \pm 0.25$ & $1.61 \pm 0.35$ & $1.23 \pm 0.25$ \\
\hline & CB 233 & $-0.77 \pm 0.05$ & $-0.77 \pm 0.05$ & $-0.77 \pm 0.05$ & $-0.76 \pm 0.06$ & $-0.71 \pm 0.06$ \\
\hline & SIL 233 & $2.57 \pm 0.86^{d}$ & $3.28 \pm 1.06^{d}$ & $3.15 \pm 1.05^{d}$ & $3.84 \pm 1.21^{d}$ & $3.12 \pm 0.99^{d}$ \\
\hline & DPM/SIL 233/233 & $5.45 \pm 0.70^{\mathrm{e}}$ & $7.02 \pm 0.79^{e}$ & $6.31 \pm 0.72^{e}$ & $9.15 \pm 1.07^{e}$ & $6.11 \pm 1.07^{e}$ \\
\hline & CB/SIL 233/233 & $0.82 \pm 0.35$ & $1.01 \pm 0.35$ & $0.91 \pm 0.36$ & $1.30 \pm 0.38$ & $1.45 \pm 0.53$ \\
\hline
\end{tabular}

All data are shown as mean fold change over control \pm standard error: ${ }^{d}$ different from control and CB; ${ }^{\text {e }}$ different from all other groups; ${ }^{9}$ different from CB only; ${ }^{j}$ different from control and both CB groups; and 'different from control, SIL, and both CB groups. Statistical significance is $p \leq 0.05$. 
Table 3: Inflammatory Proteins in BALF

\begin{tabular}{|c|c|c|c|c|c|c|c|c|}
\hline \multirow{7}{*}{ 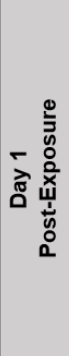 } & $\begin{array}{l}\text { Exposure } \\
\text { Group }(\mu \mathrm{g})\end{array}$ & LIX & MCP-1 & MIP-1 $1 \alpha$ & MIP-2 & IL-6 & IL-18 & TNF- $\alpha$ \\
\hline & CONTROL & $36.87 \pm 4.10$ & $7.69 \pm 2.84$ & $3.93 \pm 0.40$ & $187.63 \pm 25.95$ & $474.40 \pm 82.14$ & $573.04 \pm 39.33$ & $2.04 \pm 0.26$ \\
\hline & DPM 233 & $54.45 \pm 4.79$ & $18.02 \pm 4.18$ & $8.87 \pm 0.81^{b}$ & $106.78 \pm 10.30$ & $378.34 \pm 59.64$ & $1047.24 \pm 83.57^{p}$ & $3.92 \pm 0.47^{b}$ \\
\hline & CB 233 & $116.31 \pm 41.51$ & $51.67 \pm 6.52^{b}$ & $15.29 \pm 3.20^{b}$ & $334.30 \pm 33.09^{n}$ & $1025.81 \pm 269.74$ & $956.72 \pm 80.37$ & $4.36 \pm 0.62^{b}$ \\
\hline & SIL 233 & $268.36 \pm 117.84$ & $28.98 \pm 5.45$ & $17.07 \pm 1.50^{i}$ & $337.55 \pm 51.09^{\circ}$ & $696.40 \pm 114.40$ & $681.91 \pm 45.37$ & $3.50 \pm 0.33^{b}$ \\
\hline & $\begin{array}{l}\text { DPM/SIL } \\
233 / 233\end{array}$ & $55.95 \pm 5.05$ & $32.96 \pm 9.42$ & $14.54 \pm 1.56^{i}$ & $88.03 \pm 7.93$ & $673.13 \pm 243.07$ & $919.03 \pm 67.74^{a}$ & $6.16 \pm 0.59^{e}$ \\
\hline & CB/SIL 233/233 & $70.29 \pm 29.73$ & $66.24 \pm 18.82^{m}$ & $14.71 \pm 1.71^{b}$ & $290.35 \pm 41.27^{n}$ & $840.95 \pm 114.62$ & $797.56 \pm 53.56^{b}$ & $4.43 \pm 0.57^{b}$ \\
\hline \multirow{6}{*}{ 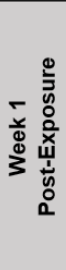 } & CONTROL & $75.43 \pm 25.96$ & $8.34 \pm 2.47$ & $3.62 \pm 0.42$ & $222.07 \pm 27.34$ & $445.28 \pm 95.57$ & $446.08 \pm 24.95$ & $1.49 \pm 0.23$ \\
\hline & DPM 233 & $56.60 \pm 9.53$ & $0.92 \pm 0.91$ & $4.69 \pm 0.56$ & $115.95 \pm 10.88$ & $455.41 \pm 81.17$ & $670.80 \pm 76.11^{d}$ & $2.54 \pm 0.36$ \\
\hline & CB 233 & $53.70 \pm 13.69$ & $3.77 \pm 1.13$ & $4.46 \pm 0.50$ & $288.24 \pm 27.40$ & $693.93 \pm 116.76$ & $390.25 \pm 27.29$ & $1.05 \pm 0.16$ \\
\hline & SIL 233 & $336.95 \pm 117.71$ & $26.23 \pm 6.94$ & $16.47 \pm 2.15^{f}$ & $312.34 \pm 45.43^{\circ}$ & $463.58 \pm 87.51$ & $732.18 \pm 32.99^{d}$ & $2.16 \pm 0.32$ \\
\hline & $\begin{array}{c}\text { DPM/SIL } \\
233 / 233\end{array}$ & $220.87 \pm 86.84$ & $52.46 \pm 17.10^{b}$ & $14.55 \pm 2.89^{f}$ & $132.78 \pm 9.96$ & $380.17 \pm 71.15$ & $835.96 \pm 78.73^{d}$ & $3.01 \pm 0.56^{\mathrm{d}}$ \\
\hline & CB/SIL 233/233 & $453.19 \pm 222.40$ & $123.20 \pm 30.73^{e}$ & $13.47 \pm 2.55^{f}$ & $488.04 \pm 45.94^{e}$ & $476.23 \pm 107.40$ & $665.42 \pm 60.41^{d}$ & $2.12 \pm 0.11$ \\
\hline \multirow{6}{*}{ 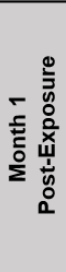 } & CONTROL & $43.98 \pm 2.68$ & $8.92 \pm 3.06$ & $4.23 \pm 0.29$ & $242.66 \pm 36.80$ & $484.10 \pm 100.09$ & $428.77 \pm 27.60$ & $1.40 \pm 0.26$ \\
\hline & DPM 233 & $61.08 \pm 11.79$ & $11.62 \pm 3.42$ & $4.44 \pm 0.46$ & $132.28 \pm 17.07$ & $339.16 \pm 54.93$ & $553.49 \pm 131.40$ & $2.19 \pm 0.38$ \\
\hline & CB 233 & $47.52 \pm 9.96$ & $4.23 \pm 2.08$ & $4.66 \pm 0.81$ & $360.43 \pm 49.01^{\circ}$ & $705.02 \pm 160.38$ & $421.00 \pm 39.64$ & $1.40 \pm 0.41$ \\
\hline & SIL 233 & $247.87 \pm 78.46^{b}$ & $48.29 \pm 5.96$ & $21.07 \pm 2.75^{f}$ & $351.43 \pm 50.66^{n}$ & $443.41 \pm 105.22$ & $570.59 \pm 53.94$ & $2.43 \pm 0.25^{q}$ \\
\hline & $\begin{array}{l}\text { DPM/SIL } \\
233 / 233\end{array}$ & $88.24 \pm 15.48$ & $176.37 \pm 41.17^{c}$ & $17.03 \pm 1.11^{f}$ & $136.37 \pm 8.64$ & $260.94 \pm 65.01$ & $920.50 \pm 79.69^{e}$ & $2.77 \pm 0.25^{q}$ \\
\hline & CB/SIL 233/233 & $281.48 \pm 79.89^{b}$ & $207.28 \pm 36.14^{c}$ & $18.65 \pm 1.80^{f}$ & $465.46 \pm 35.14^{n}$ & $757.55 \pm 163.67$ & $695.14 \pm 62.20$ & $1.40 \pm 0.23$ \\
\hline \multirow{6}{*}{ 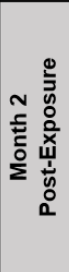 } & CONTROL & $40.57 \pm 4.03$ & $10.22 \pm 2.94$ & $3.40 \pm 0.34$ & $261.32 \pm 33.05$ & $373.85 \pm 84.79$ & $412.89 \pm 34.56$ & $1.42 \pm 0.19$ \\
\hline & DPM 233 & $310.50 \pm 148.22$ & $13.5799 \pm 4.63$ & $18.73 \pm 2.74$ & $158.95 \pm 13.87$ & $87.36 \pm 16.97$ & $883.66 \pm 121.34^{p}$ & $3.43 \pm 0.37^{e}$ \\
\hline & CB 233 & $38.24 \pm 9.11$ & $7.20 \pm 2.50$ & $3.56 \pm 0.37$ & $374.00 \pm 46.57^{\circ}$ & $481.64 \pm 67.07$ & $404.85 \pm 57.82$ & $0.65 \pm 0.15$ \\
\hline & SIL 233 & $209.76 \pm 83.77$ & $141.24 \pm 28.09^{i}$ & $22.38 \pm 5.88^{d}$ & $346.60 \pm 48.69^{\circ}$ & $416.06 \pm 121.45$ & $536.72 \pm 74.46$ & $1.81 \pm 0.33^{g}$ \\
\hline & $\begin{array}{l}\text { DPM/SIL } \\
233 / 233\end{array}$ & $254.36 \pm 66.38$ & $300.30 \pm 103.22^{c}$ & $28.30 \pm 7.13^{d}$ & $129.70 \pm 8.49$ & $81.40 \pm 10.24$ & $730.30 \pm 109.70^{b}$ & $2.35 \pm 0.45^{g}$ \\
\hline & CB/SIL 233/233 & $295.98 \pm 201.98$ & $277.12 \pm 84.97^{c}$ & $24.65 \pm 6.98^{d}$ & $496.50 \pm 14.02^{m}$ & $614.00 \pm 62.65^{\circ}$ & $760.10 \pm 96.05^{d}$ & $1.43 \pm 0.33$ \\
\hline \multirow{6}{*}{ 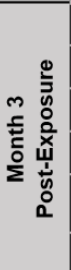 } & CONTROL & $55.012 \pm 10.33$ & $23.41 \pm 2.80$ & $5.18 \pm 1.77$ & $287.20 \pm 33.59^{\circ}$ & $466.81 \pm 133.09$ & $490.84 \pm 77.07$ & $1.41 \pm 0.22$ \\
\hline & DPM 233 & $66.77 \pm 13.05$ & $11.83 \pm 3.35$ & $12.38 \pm 2.79$ & $163.11 \pm 15.46$ & $76.94 \pm 30.91$ & $581.54 \pm 123.05$ & $2.18 \pm 0.17$ \\
\hline & CB 233 & $55.71 \pm 9.37$ & $17.79 \pm 4.14$ & $4.20 \pm 0.50$ & $386.04 \pm 21.82^{\circ}$ & $666.59 \pm 156.33$ & $364.41 \pm 26.07$ & $1.46 \pm 0.49$ \\
\hline & SIL 233 & $540.25 \pm 180.43^{b}$ & $248.09 \pm 49.26$ & $31.89 \pm 10.01^{d}$ & $388.21 \pm 46.46^{\circ}$ & $432.65 \pm 152.47$ & $641.84 \pm 93.03$ & $1.83 \pm 0.43$ \\
\hline & $\begin{array}{c}\text { DPM/SIL } \\
233 / 233\end{array}$ & $303.29 \pm 95.71$ & $375.09 \pm 132.55^{f}$ & $23.73 \pm 6.49$ & $147.16 \pm 9.25$ & $66.28 \pm 12.91$ & $813.91 \pm 177.93$ & $2.20 \pm 0.34$ \\
\hline & CB/SIL 233/233 & $571.78 \pm 160.46^{b}$ & $197.83 \pm 49.98^{f}$ & $38.77 \pm 7.20^{d}$ & $505.53 \pm 21.76^{n}$ & $1052.36 \pm 272.45^{\mathrm{m}}$ & $955.12 \pm 82.81^{d}$ & $2.03 \pm 0.68$ \\
\hline
\end{tabular}

All data are shown as mean \pm standard error: ${ }^{a}$ different than control and SIL groups; ${ }^{\mathbf{b}}$ different from control only; ${ }^{\mathrm{c}}$ different from control and all single dose groups; ${ }^{\mathrm{d}}$ different from control and CB; ${ }^{\mathrm{e}}$ different from all other groups; ${ }^{f}$ different from control, DPM and CB; ${ }^{9}$ different from CB only; ${ }^{i}$ different from control and DPM groups; ${ }^{m}$ different from control, SIL, and both DPM groups; ${ }^{n}$ different from control both DPM groups; ${ }^{\circ}$ different from both DPM groups only; ${ }^{\mathfrak{p}}$ different from control, SIL, and $\mathrm{CB} / \mathrm{SIL}$; and ${ }^{9}$ different from control and CB/SIL groups. Statistical significance is $p \leq 0.05$. 
Table 4: Immuno-regulatory Proteins in BALF

\begin{tabular}{|c|c|c|c|c|c|c|c|}
\hline \multirow{7}{*}{ 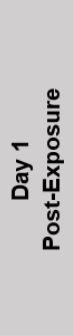 } & Exposure Group $(\mu \mathrm{g})$ & IL-2 & IL-4 & IL-10 & IL-12p70 & IFN-Y & IP-10 \\
\hline & CONTROL & $32.10 \pm 7.25$ & $13.42 \pm 1.85$ & $25.42 \pm 3.31$ & $35.52 \pm 10.51$ & $13.11 \pm 9.68$ & $22.16 \pm 5.72$ \\
\hline & DPM 233 & $48.41 \pm 14.56$ & $15.90 \pm 2.38$ & $17.62 \pm 3.01$ & $13.22 \pm 2.03$ & $36.96 \pm 30.01$ & $44.04 \pm 11.75$ \\
\hline & CB 233 & $26.72 \pm 5.90$ & $11.33 \pm 2.09$ & $29.03 \pm 4.75$ & $35.84 \pm 10.42$ & $37.75 \pm 17.49$ & $196.21 \pm 41.04^{h}$ \\
\hline & SIL 233 & $41.46 \pm 8.26$ & $10.59 \pm 1.56$ & $18.90 \pm 1.29$ & $28.98 \pm 6.57$ & $72.62 \pm 42.39$ & $92.82 \pm 17.27$ \\
\hline & DPM/SIL 233/233 & $68.19 \pm 14.19$ & $15.27 \pm 4.35$ & $19.29 \pm 3.49$ & $14.61 \pm 3.39$ & $11.20 \pm 8.05$ & $114.37 \pm 33.88$ \\
\hline & CB/SIL 233/233 & $30.63 \pm 11.70$ & $13.01 \pm 5.01$ & $19.71 \pm 3.76$ & $39.78 \pm 8.61$ & $37.21 \pm 23.18$ & $195.78 \pm 50.09^{h}$ \\
\hline \multirow{6}{*}{ 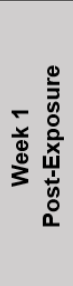 } & CONTROL & $35.97 \pm 8.67$ & $16.38 \pm 2.23$ & $15.47 \pm 2.11$ & $31.47 \pm 8.15$ & $51.93 \pm 21.72$ & $13.52 \pm 1.62$ \\
\hline & DPM 233 & $55.85 \pm 17.56$ & $17.90 \pm 2.12$ & $14.93 \pm 2.17$ & $11.32 \pm 2.90$ & $72.06 \pm 55.20$ & $12.60 \pm 2.21$ \\
\hline & CB 233 & $26.57 \pm 6.53$ & $13.52 \pm 1.81$ & $26.39 \pm 5.42$ & $58.14 \pm 16.13$ & $61.24 \pm 45.60$ & $14.81 \pm 4.09$ \\
\hline & SIL 233 & $52.35 \pm 10.35$ & $17.21 \pm 3.74$ & $22.48 \pm 3.62$ & $38.38 \pm 9.91$ & $28.72 \pm 15.20$ & $28.88 \pm 4.45$ \\
\hline & DPM/SIL 233/233 & $32.57 \pm 11.49$ & $12.49 \pm 1.95$ & $16.16 \pm 2.19$ & $14.53 \pm 1.50$ & $57.73 \pm 56.67$ & $67.62 \pm 17.93^{c}$ \\
\hline & CB/SIL 233/233 & $38.85 \pm 11.41$ & $11.24 \pm 3.14$ & $22.18 \pm 3.98$ & $36.06 \pm 6.69$ & $35.13 \pm 33.30$ & $93.59 \pm 27.70^{c}$ \\
\hline \multirow{6}{*}{ 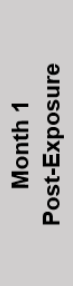 } & CONTROL & $39.02 \pm 10.01$ & $13.86 \pm 1.86$ & $21.78 \pm 4.25$ & $29.59 \pm 8.46$ & $75.31 \pm 34.28$ & $16.18 \pm 2.44$ \\
\hline & DPM 233 & $54.43 \pm 11.89$ & $13.35 \pm 1.49$ & $13.38 \pm 1.95$ & $12.34 \pm 2.14$ & $78.36 \pm 49.63$ & $11.92 \pm 2.27$ \\
\hline & CB 233 & $47.12 \pm 19.67$ & $25.01 \pm 7.42^{r}$ & $21.03 \pm 5.74$ & $55.82 \pm 14.14^{\mathrm{s}}$ & $37.01 \pm 20.94$ & $12.22 \pm 1.42$ \\
\hline & SIL 233 & $44.57 \pm 10.52$ & $11.10 \pm 2.47$ & $19.84 \pm 2.73$ & $25.08 \pm 4.53$ & $18.14 \pm 16.22$ & $26.06 \pm 3.48$ \\
\hline & DPM/SIL 233/233 & $22.11 \pm 7.66$ & $6.22 \pm 1.40$ & $14.41 \pm 1.69$ & $10.55 \pm 2.37$ & $0.93 \pm 0.34$ & $51.76 \pm 7.76^{d}$ \\
\hline & CB/SIL 233/233 & $29.07 \pm 7.66$ & $12.65 \pm 2.26$ & $17.34 \pm 3.97$ & $41.65 \pm 8.59$ & $1.83 \pm 0.00$ & $88.96 \pm 26.39^{e}$ \\
\hline \multirow{6}{*}{ 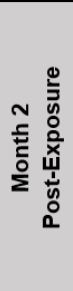 } & CONTROL & $17.41 \pm 4.62$ & $13.99 \pm 2.04$ & $15.10 \pm 1.14$ & $42.14 \pm 8.16$ & $94.74 \pm 29.59$ & $14.57 \pm 2.31$ \\
\hline & DPM 233 & $2.70 \pm 0.73$ & $3.17 \pm 0.49$ & $14.40 \pm 1.36$ & $19.73 \pm 3.52$ & $38.20 \pm 36.41$ & $74.00 \pm 22.81^{d}$ \\
\hline & СВ 233 & $26.60 \pm 7.32$ & $13.22 \pm 4.41$ & $13.15 \pm 3.03$ & $41.50 \pm 9.01$ & $7.51 \pm 5.68$ & $7.89 \pm 2.44$ \\
\hline & SIL 233 & $26.24 \pm 8.59$ & $11.16 \pm 3.23$ & $13.65 \pm 1.98$ & $38.58 \pm 7.41$ & $60.86 \pm 19.91$ & $39.63 \pm 8.69$ \\
\hline & DPM/SIL 233/233 & $2.35 \pm 0.38$ & $4.30 \pm 0.90$ & $13.42 \pm 2.94$ & $23.02 \pm 4.51$ & $63.95 \pm 39.36$ & $48.11 \pm 11.39$ \\
\hline & CB/SIL 233/233 & $16.65 \pm 2.95$ & $9.02 \pm 2.26$ & $8.64 \pm 1.88$ & $45.54 \pm 7.77$ & $2.73 \pm 0.90$ & $39.56 \pm 11.06$ \\
\hline \multirow{6}{*}{ 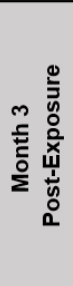 } & CONTROL & $25.89 \pm 10.15$ & $12.91 \pm 4.24$ & $12.01 \pm 1.35$ & $30.50 \pm 4.26$ & $23.37 \pm 13.25$ & $16.59 \pm 3.32$ \\
\hline & DPM 233 & $2.53 \pm 0.54$ & $3.07 \pm 2.08$ & $7.34 \pm 0.93$ & $23.58 \pm 3.01$ & $14.33 \pm 12.50$ & $40.77 \pm 10.66$ \\
\hline & CB 233 & $49.97 \pm 16.22$ & $22.99 \pm 5.54$ & $22.35 \pm 4.37$ & $40.10 \pm 5.94$ & $59.23 \pm 37.62$ & $15.81 \pm 2.40$ \\
\hline & SIL 233 & $25.28 \pm 9.40$ & $13.45 \pm 2.69$ & $18.82 \pm 5.32$ & $38.50 \pm 8.31$ & $55.70 \pm 20.24$ & $51.21 \pm 11.63$ \\
\hline & DPM/SIL 233/233 & $2.94 \pm 0.69$ & $3.13 \pm 1.11$ & $9.97 \pm 1.26$ & $21.16 \pm 3.79$ & $16.39 \pm 13.83$ & $47.74 \pm 8.77$ \\
\hline & CB/SIL 233/233 & $41.29 \pm 20.12$ & $18.79 \pm 9.29$ & $28.65 \pm 6.48^{n}$ & $41.10 \pm 8.36$ & $68.19 \pm 31.28$ & $90.83 \pm 33.94^{d}$ \\
\hline
\end{tabular}

All data are shown as mean \pm standard error: $^{\mathbf{c}}$ different from control and all single dose groups; d different from control and CB; ${ }^{\mathrm{e}}$ different from all other groups; ${ }^{\mathrm{h}}$ different from control, DPM, and SIL groups; ${ }^{n}$ different from control and both DPM groups; ${ }^{r}$ different from control, SIL, and DPM/SIL groups; and sdifferent from SIL and both DPM groups. Statistical significance is $p \leq$ 0.05 . 
Table 5: Tissue Remodeling/Wound Healing Proteins in BALF

\begin{tabular}{|c|c|c|c|c|c|}
\hline \multirow{7}{*}{ 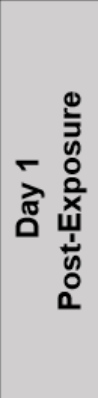 } & $\begin{array}{c}\text { Exposure Group } \\
\text { (uq) }\end{array}$ & MMP-9 & OPN & TGF $\beta 2$ & TIMP-1 \\
\hline & CONTROL & $0.30 \pm 0.12$ & $6.67 \pm 1.81$ & $1403.53 \pm 238.19$ & $4484.93 \pm 334.06$ \\
\hline & DPM 233 & $4.49 \pm 1.11$ & $10.80 \pm 1.52$ & $2484.21 \pm 392.32^{b}$ & $5591.61 \pm 275.70$ \\
\hline & СВ 233 & $12.43 \pm 3.12^{b}$ & $32.00 \pm 6.67^{m}$ & $2631.13 \pm 293.08^{b}$ & $5887.72 \pm 369.88^{b}$ \\
\hline & SIL 233 & $11.26 \pm 2.92^{\mathrm{b}}$ & $15.44 \pm 3.42$ & $2911.33 \pm 225.00^{b}$ & $4873.78 \pm 219.16$ \\
\hline & DPM/SIL 233/233 & $7.80 \pm 1.92$ & $12.09 \pm 2.83$ & $3010.96 \pm 274.59^{b}$ & $6156.47 \pm 322.66^{a}$ \\
\hline & CB/SIL 233/233 & $9.53 \pm 3.15^{b}$ & $37.10 \pm 7.48^{m}$ & $2447.56 \pm 328.07^{b}$ & $5438.87 \pm 261.19$ \\
\hline \multirow{6}{*}{ 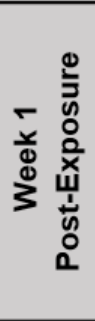 } & CONTROL & $0.24 \pm 0.08$ & $8.32 \pm 1.51$ & $974.56 \pm 144.63$ & $1914.68 \pm 98.12$ \\
\hline & DPM 233 & $0.20 \pm 0.04$ & $5.14 \pm 0.94$ & $1181.74 \pm 224.71$ & $1996.80 \pm 174.68$ \\
\hline & CB 233 & $0.06 \pm 0.03$ & $8.14 \pm 2.58$ & $744.85 \pm 111.42$ & $2220.00 \pm 128.55$ \\
\hline & SIL 233 & $0.88 \pm 0.15$ & $13.73 \pm 1.92$ & $985.08 \pm 97.40$ & $1982.64 \pm 71.75$ \\
\hline & DPM/SIL 233/233 & $2.79 \pm 0.87^{c}$ & $14.41 \pm 2.27$ & $1583.73 \pm 219.63^{g}$ & $2223.06 \pm 160.42$ \\
\hline & CB/SIL 233/233 & $2.41 \pm 0.62^{c}$ & $48.50 \pm 13.01^{e}$ & $1438.78 \pm 206.31$ & $2340.90 \pm 111.92$ \\
\hline \multirow{6}{*}{ 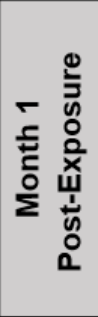 } & CONTROL & $0.12 \pm 0.03$ & $12.50 \pm 2.49$ & $1171.08 \pm 130.83$ & $1694.32 \pm 77.88$ \\
\hline & DPM 233 & $0.17 \pm 0.08$ & $5.80 \pm 1.38$ & $1082.30 \pm 161.09$ & $1915.37 \pm 111.27$ \\
\hline & CB 233 & $0.06 \pm 0.03$ & $10.30 \pm 2.13$ & $1107.29 \pm 182.47$ & $2031.15 \pm 253.78$ \\
\hline & SIL 233 & $1.38 \pm 0.18^{f}$ & $16.82 \pm 2.34$ & $956.25 \pm 69.17$ & $1814.10 \pm 91.42$ \\
\hline & DPM/SIL 233/233 & $2.64 \pm 0.40^{c}$ & $35.05 \pm 7.24^{c}$ & $2139.44 \pm 181.06^{e}$ & $2705.41 \pm 174.58^{\mathrm{e}}$ \\
\hline & CB/SIL 233/233 & $3.13 \pm 0.35^{c}$ & $47.90 \pm 13.85^{c}$ & $1444.96 \pm 115.70$ & $1767.84 \pm 87.92$ \\
\hline \multirow{6}{*}{ 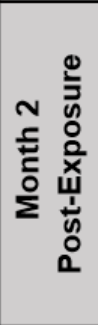 } & CONTROL & $0.06 \pm 0.02$ & $14.14 \pm 2.01$ & $1566.93 \pm 195.92$ & $1801.45 \pm 73.379$ \\
\hline & DPM 233 & $0.14 \pm 0.09$ & $7.39 \pm 0.46$ & $1527.17 \pm 354.91$ & $1948.98 \pm 116.979$ \\
\hline & CB 233 & $0.08 \pm 0.04$ & $6.87 \pm 3.33$ & $897.68 \pm 164.92$ & $1472.62 \pm 87.39$ \\
\hline & SIL 233 & $2.94 \pm 0.42^{f}$ & $28.55 \pm 3.69^{f}$ & $1967.10 \pm 237.62$ & $2061.41 \pm 85.35^{9}$ \\
\hline & DPM/SIL 233/233 & $2.98 \pm 0.42^{f}$ & $40.22 \pm 7.39^{f}$ & $2150.47 \pm 329.57$ & $2347.79 \pm 89.24^{d}$ \\
\hline & CB/SIL 233/233 & $3.23 \pm 0.42^{f}$ & $38.09 \pm 11.37^{f}$ & $1710.53 \pm 283.69$ & $1964.24 \pm 138.179$ \\
\hline \multirow{6}{*}{ 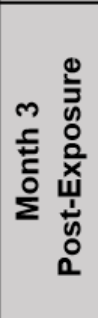 } & CONTROL & $0.26 \pm 0.12$ & $14.37 \pm 2.24$ & $1443.38 \pm 172.76$ & $2231.01 \pm 193.83$ \\
\hline & DPM 233 & $0.17 \pm 0.03$ & $7.47 \pm 0.59$ & $1948.96 \pm 180.33$ & $2118.38 \pm 41.41$ \\
\hline & CB 233 & $0.20 \pm 0.12$ & $8.55 \pm 2.03$ & $1296.49 \pm 128.52$ & $1580.71 \pm 110.83$ \\
\hline & SIL 233 & $4.79 \pm 0.70^{f}$ & $40.77 \pm 6.21^{f}$ & $1898.74 \pm 188.95$ & $2243.83 \pm 175.83$ \\
\hline & DPM/SIL 233/233 & $4.84 \pm 1.30^{f}$ & $45.18 \pm 6.76^{f}$ & $3081.42 \pm 144.32^{e}$ & $2290.31 \pm 166.01$ \\
\hline & CB/SIL 233/233 & $4.10 \pm 0.91^{f}$ & $49.18 \pm 11.79^{f}$ & $1970.60 \pm 296.53$ & $2103.03 \pm 170.40$ \\
\hline
\end{tabular}

All data are shown as mean \pm standard error: ${ }^{a}$ different than control and SIL groups; ${ }^{b}$ different from control only; ' different from control and all single dose groups; ${ }^{d}$ different from control and CB; ${ }^{e}$ different from all other groups; ${ }^{f}$ different from control, DPM and CB; 9 different from CB only; and ${ }^{\mathrm{m}}$ different from control, SIL, and both DPM groups. Statistical significance is $\mathrm{p} \leq 0.05$. 
Table 6: Severity and Incidence Scores for Lung Pathology

\begin{tabular}{|c|c|c|c|c|c|}
\hline Exposure Group ( $\mu \mathrm{g})$ & $\begin{array}{l}\text { Time Post- } \\
\text { Exposure }\end{array}$ & AM & AEH & BEH & $\mathbf{F}$ \\
\hline Control & 1 week & $0.5(5 / 10) \mathrm{D}$ & $0(0 / 10)$ & $0(0 / 10)$ & $0(0 / 10)$ \\
\hline DPM 233 & 1 week & $0.8(4 / 5) \mathrm{D}$ & $0(0 / 5)$ & $0(0 / 5)$ & $0(0 / 5)$ \\
\hline СВ 233 & 1 week & $1(5 / 5) \mathrm{D}$ & $0(0 / 5)$ & $0(0 / 5)$ & $0(0 / 5)$ \\
\hline SIL 233 & 1 week & $1.4(10 / 10) D^{b *}$ & $0(0 / 10)$ & $0(0 / 10)$ & $0(0 / 10)$ \\
\hline DPM/SIL 233/233 & 1 week & $1(3 / 5) \mathrm{D}$ & $0.2(1 / 5) \mathrm{M}$ & $0(0 / 5)$ & $0(0 / 5)$ \\
\hline CB/SIL 233/233 & 1 week & $1.8(5 / 5) M^{f *}$ & $0(0 / 5)$ & $0(0 / 5)$ & $0(0 / 5)$ \\
\hline Control & 1 month & $0.8(8 / 10) D^{g}$ & $0(0 / 10)$ & $0(0 / 10)$ & $0.1(1 / 10) \mathrm{D}$ \\
\hline DPM 233 & 1 month & $1(5 / 5) \mathrm{Dg}$ & $0(0 / 5)$ & $0(0 / 5)$ & $0(0 / 5)$ \\
\hline CB 233 & 1 month & $0.2(1 / 5) \mathrm{D}$ & $0(0 / 5)$ & $0(0 / 5)$ & $0(0 / 5)$ \\
\hline SIL 233 & 1 month & $1.4(9 / 10) D^{d}$ & $0.2(2 / 10) \mathrm{M}$ & $0(0 / 10)$ & $0.2(2 / 10) \mathrm{D}$ \\
\hline DPM/SIL 233/233 & 1 month & $1.4(5 / 5) D^{d}$ & $1.4(5 / 5) M^{c * *}$ & $0.8(3 / 5) M^{a}$ & $0.2(1 / 5) \mathrm{D}$ \\
\hline CB/SIL 233/233 & 1 month & $2(5 / 5) M^{f * *}$ & $0.6(3 / 5) M^{b}$ & $0.8(4 / 5) M^{c \dagger}$ & $0.6(3 / 5) \mathrm{M}$ \\
\hline Control & 2 months & $0.7(7 / 10) \mathrm{D}$ & $0(0 / 10)$ & $0(0 / 10)$ & $0(0 / 10)$ \\
\hline DPM 233 & 2 months & $1.2(5 / 5) \mathrm{D}$ & $0(0 / 5)$ & $0(0 / 5)$ & $0(0 / 5)$ \\
\hline CВ 233 & 2 months & $1(5 / 5) \mathrm{D}$ & $0(0 / 5)$ & $0(0 / 5)$ & $0(0 / 5)$ \\
\hline SIL 233 & 2 months & $1.6(10 / 10) M^{d *}$ & $0.7(7 / 10) M^{f *}$ & $0.4(4 / 10) M^{b}$ & $0.3(3 / 10) \mathrm{D}$ \\
\hline DPM/SIL 233/233 & 2 months & $1.8(5 / 5) M^{d *}$ & $1.2(5 / 5) M^{f *}$ & $0.8(4 / 5) M^{f *}$ & $0.8(4 / 5) M^{f *}$ \\
\hline CB/SIL 233/233 & 2 months & $1.6(5 / 5) \mathrm{M}^{\mathrm{b}}$ & $0.8(4 / 5) M^{f *}$ & $0.4(2 / 5) M^{b}$ & $0.8(4 / 5) M^{f *}$ \\
\hline Control & 3 months & $0.6(6 / 10) \mathrm{D}$ & $0(0 / 10)$ & $0(0 / 10)$ & $0(0 / 10)$ \\
\hline DPM 233 & 3 months & $0.8(4 / 5) \mathrm{D}$ & $0(0 / 5)$ & $0(0 / 5)$ & $0(0 / 5)$ \\
\hline CВ 233 & 3 months & $1(5 / 5) \mathrm{D}$ & $0(0 / 5)$ & $0(0 / 5)$ & $0(0 / 5)$ \\
\hline SIL 233 & 3 months & $1.6(10 / 10) M^{f *}$ & $1.1(9 / 10) M^{f * *}$ & $0.6(6 / 10) M^{f *}$ & $0.6(6 / 10) D^{f *}$ \\
\hline DPM/SIL 233/233 & 3 months & $2(5 / 5) M^{f \star \star}$ & $1.8(5 / 5) \mathrm{M}^{\mathrm{ctt}}$ & $0.6(3 / 5) M^{b}$ & $0.8(4 / 5) M^{f *}$ \\
\hline CB/SIL 233/233 & 3 months & $1.8(5 / 5) M^{f *}$ & $1.2(5 / 5) M^{f * *}$ & $0.2(1 / 5) \mathrm{M}$ & $1.0(5 / 5) M^{f \star *}$ \\
\hline
\end{tabular}

Lung tissue was analyzed and scored for accumulation of alveolar macrophages as an index of inflammation (AM), alveolar epithelial hyperplasia $(\mathrm{AEH})$, bronchial epithelial hyperplasia $(\mathrm{BEH})$, and fibrosis in alveolar septae (F) ( $n=5$ for CB, DPM, DPM/SIL, and CB/SIL groups and $n=10$ per PBS and SIL per time point). Severity was scored as $0-5: 0=$ normal, $1=$ minimal/slight, 2 = mild, 3 = moderate, 4 = marked, and 5 = severe. Incidence was noted as Focal (F), Multi-focal $(\mathrm{M})$, and Diffuse (D). Data are presented as means with incidence (number of animals with a positive score per total animals) in parentheses. ${ }^{\mathbf{a}}$ different than control and SIL groups; ${ }^{\mathbf{b}}$ different from control only; ${ }^{c}$ different from control and all single dose groups; ${ }^{d}$ different from control and CB; ${ }^{f}$ different from control, DPM and CB; and ${ }^{g}$ different from CB only; Note: all letter symbol notations indicate a $p$ value $<0.05$ except where a single asterisk indicates $p<$ 0.05 for all groups except control $<0.01$, a double asterisk indicates $p<0.01$ for all groups. A single cross indicates $p<0.05$ for all groups except control and SIL where $p<0.01$, and a double cross indicates $p<0.05$ for SIL and $p<0.01$ for all other groups. 
Table 7: Lymphocyte Phenotype of BALC Following LM Infection

\begin{tabular}{|c|c|c|c|c|c|}
\hline \multirow{6}{*}{ 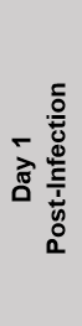 } & Exposure $(\mu \mathrm{g})$ & T Cells & $\mathrm{CD4}^{+} \mathrm{T}$ Cells & CD8 ${ }^{+}$T Cells & B Cells \\
\hline & DPM 233 & $0.43 \pm 0.46$ & $0.51 \pm 0.50$ & $0.23 \pm 0.38$ & $0.003 \pm 0.24$ \\
\hline & CB 233 & $-0.09 \pm 0.33$ & $-0.04 \pm 0.36$ & $-0.27 \pm 0.22$ & $-0.27 \pm 0.29$ \\
\hline & SIL 233 & $0.81 \pm 0.27$ & $0.81 \pm 0.26$ & $0.71 \pm 0.34$ & $0.54 \pm 0.32$ \\
\hline & DPM/SIL 233/233 & $0.77 \pm 0.21$ & $0.90 \pm 0.22$ & $0.30 \pm 0.23$ & $0.10 \pm 0.16$ \\
\hline & CB/SIL 233/233 & $0.80 \pm 0.14$ & $0.95 \pm 0.16$ & $-0.08 \pm 0.10$ & $-0.22 \pm 0.08$ \\
\hline \multirow{5}{*}{ 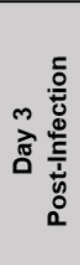 } & DPM 233 & $-0.05 \pm 0.10$ & $0.06 \pm 0.11$ & $-0.16 \pm 0.12$ & $0.06 \pm 0.20$ \\
\hline & CB 233 & $-0.53 \pm 0.07$ & $-0.47 \pm 0.07$ & $-0.44 \pm 0.10$ & $-0.71 \pm 0.04$ \\
\hline & SIL 233 & $-0.44 \pm 0.09$ & $-0.22 \pm 0.09$ & $-0.51 \pm 0.09^{E}$ & $-0.39 \pm 0.12$ \\
\hline & DPM/SIL 233/233 & $-0.14 \pm 0.10$ & $0.39 \pm 0.16^{\prime}$ & $-0.37 \pm 0.09$ & $-0.12 \pm 0.11$ \\
\hline & CB/SIL 233/233 & $-0.50 \pm 0.11$ & $0.01 \pm 0.20$ & $-0.65 \pm 0.10^{\mathrm{E}}$ & $-0.79 \pm 0.04$ \\
\hline \multirow{5}{*}{ n } & DPM 233 & $0.34 \pm 0.26$ & $0.15 \pm 0.20$ & $0.43 \pm 0.29$ & $0.003 \pm 0.09$ \\
\hline & CB 233 & $-0.02 \pm 0.08$ & $-0.01 \pm 0.09$ & $-0.01 \pm 0.08$ & $0.16 \pm 0.13$ \\
\hline & SIL 233 & $-0.24 \pm 0.10$ & $-0.31 \pm 0.09$ & $-0.18 \pm 0.11$ & $-0.19 \pm 0.14$ \\
\hline & DPM/SIL 233/233 & $-0.13 \pm 0.18$ & $-0.20 \pm 0.20$ & $-0.08 \pm 0.17$ & $-0.23 \pm 0.17$ \\
\hline & CB/SIL 233/233 & $-0.51 \pm 0.05^{c}$ & $-0.38 \pm 0.07$ & $-0.56 \pm 0.05^{c}$ & $-0.41 \pm 0.07$ \\
\hline \multirow{5}{*}{ ヘ } & DPM 233 & $0.21 \pm 0.23$ & $0.03 \pm 0.20$ & $0.29 \pm 0.28$ & $0.02 \pm 0.16$ \\
\hline & CB 233 & $0.07 \pm 0.16$ & $0.08 \pm 0.21$ & $0.08 \pm 0.15$ & $0.02 \pm 0.16$ \\
\hline & SIL 233 & $-0.07 \pm 0.11$ & $-0.10 \pm 0.11$ & $-0.10 \pm 0.12$ & $0.06 \pm 0.14$ \\
\hline & DPM/SIL 233/233 & $-0.23 \pm 0.15$ & $-0.38 \pm 0.11$ & $-0.21 \pm 0.19$ & $-0.42 \pm 0.07$ \\
\hline & CB/SIL 233/233 & $-0.37 \pm 0.11$ & $-0.24 \pm 0.11$ & $-0.50 \pm 0.09$ & $-0.29 \pm 0.12$ \\
\hline \multirow{5}{*}{ 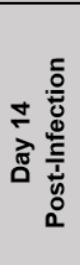 } & DPM 233 & $1.27 \pm 0.46^{\mathrm{E}}$ & $1.82 \pm 0.75$ & $1.08 \pm 0.29$ & $0.26 \pm 0.11$ \\
\hline & CB 233 & $0.12 \pm 0.24$ & $0.15 \pm 0.49$ & $0.11 \pm 0.21$ & $-0.43 \pm 0.07$ \\
\hline & SIL 233 & $0.48 \pm 0.20$ & $1.03 \pm 0.27$ & $0.63 \pm 0.26$ & $0.05 \pm 0.12$ \\
\hline & DPM/SIL 233/233 & $1.50 \pm 0.37^{D}$ & $2.77 \pm 0.62^{D}$ & $1.56 \pm 0.43^{E}$ & $0.38 \pm 0.27^{\mathrm{K}}$ \\
\hline & CB/SIL 233/233 & $2.63 \pm 0.66^{G}$ & $4.67 \pm 1.19^{G}$ & $2.24 \pm 0.99^{J}$ & $0.21 \pm 0.25$ \\
\hline
\end{tabular}

All data are shown as mean fold change over control \pm standard error: ${ }^{\mathrm{C}}$ different than control, DPM, and CB groups; ${ }^{D}$ different from control and CB groups; ${ }^{\mathrm{E}}$ different from control only; ${ }^{\mathrm{G}}$ different from all other groups; ' different from CB and SIL groups; ' different from control, CB, and SIL groups; and ${ }^{\mathrm{K}}$ different from CB only. Statistical significance is $p \leq 0.05$. 
Table 8: Lymphocyte Phenotype in the MLN Following LM Infection

\begin{tabular}{|c|c|c|c|c|c|}
\hline \multirow{6}{*}{ 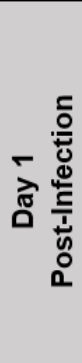 } & Exposure $(\mu \mathrm{g})$ & T Cells & $\mathrm{CD4}^{+} \mathrm{T}$ Cells & CD8+ T Cells & B Cells \\
\hline & DPM 233 & $0.07 \pm 0.09$ & $0.11 \pm 0.10$ & $0.01 \pm 0.09$ & $0.26 \pm 0.12$ \\
\hline & CB 233 & $0.41 \pm 0.30$ & $0.44 \pm 0.27$ & $0.30 \pm 0.33$ & $0.25 \pm 0.25$ \\
\hline & SIL 233 & $0.94 \pm 0.19^{B}$ & $0.91 \pm 0.18^{B}$ & $0.82 \pm 0.19^{E}$ & $1.00 \pm 0.20^{E}$ \\
\hline & DPM/SIL 233/233 & $1.45 \pm 0.38^{A}$ & $1.50 \pm 0.36^{A}$ & $1.44 \pm 0.45^{A}$ & $1.16 \pm 0.25^{\mathrm{E}}$ \\
\hline & CB/SIL 233/233 & $2.03 \pm 0.42^{F}$ & $2.12 \pm 0.42^{F}$ & $1.70 \pm 0.43^{A}$ & $2.07 \pm 0.47^{c}$ \\
\hline \multirow{5}{*}{ m } & DPM 233 & $0.39 \pm 0.11$ & $0.47 \pm 0.11$ & $0.29 \pm 0.11$ & $0.31 \pm 0.21$ \\
\hline & CB 233 & $0.38 \pm 0.14$ & $0.49 \pm 0.13$ & $0.22 \pm 0.17$ & $0.29 \pm 0.21$ \\
\hline & SIL 233 & $0.14 \pm 0.10$ & $0.23 \pm 0.11$ & $-0.01 \pm 0.08$ & $0.25 \pm 0.09$ \\
\hline & DPM/SIL 233/233 & $0.29 \pm 0.12$ & $0.44 \pm 0.14$ & $0.12 \pm 0.10$ & $0.55 \pm 0.20$ \\
\hline & CB/SIL 233/233 & $0.74 \pm 0.17^{\mathrm{H}}$ & $0.99 \pm 0.23^{\mathrm{H}}$ & $0.39 \pm 0.10$ & $1.15 \pm 0.25^{\mathrm{C}}$ \\
\hline \multirow{5}{*}{ 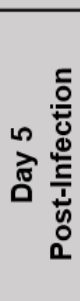 } & DPM 233 & $0.12 \pm 0.21$ & $0.13 \pm 0.23$ & $0.11 \pm 0.18$ & $0.20 \pm 0.27$ \\
\hline & CB 233 & $0.26 \pm 0.21$ & $0.28 \pm 0.22$ & $0.21 \pm 0.20$ & $0.33 \pm 0.21$ \\
\hline & SIL 233 & $-0.11 \pm 0.08$ & $-0.14 \pm 0.07$ & $-0.08 \pm 0.09$ & $-0.16 \pm 0.07$ \\
\hline & DPM/SIL 233/233 & $0.29 \pm 0.14$ & $0.24 \pm 0.15$ & $0.33 \pm 0.14$ & $0.22 \pm 0.16$ \\
\hline & CB/SIL 233/233 & $0.39 \pm 0.18$ & $0.43 \pm 0.19$ & $0.32 \pm 0.18$ & $0.51 \pm 0.21$ \\
\hline \multirow{5}{*}{ 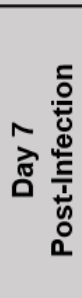 } & DPM 233 & $0.15 \pm 0.19$ & $0.16 \pm 0.21$ & $0.13 \pm 0.15$ & $0.001 \pm 0.17$ \\
\hline & CB 233 & $0.53 \pm 0.16$ & $0.47 \pm 0.15$ & $0.59 \pm 0.20$ & $0.66 \pm 0.15$ \\
\hline & SIL 233 & $0.16 \pm 0.13$ & $0.17 \pm 0.14$ & $0.10 \pm 0.12$ & $0.06 \pm 0.11$ \\
\hline & DPM/SIL 233/233 & $0.25 \pm 0.14$ & $0.32 \pm 0.14$ & $0.19 \pm 0.14$ & $-0.03 \pm 0.13$ \\
\hline & CB/SIL 233/233 & $0.74 \pm 0.28 \mathrm{E}$ & $0.69 \pm 0.28$ & $0.66 \pm 0.28$ & $0.64 \pm 0.32$ \\
\hline \multirow{5}{*}{ 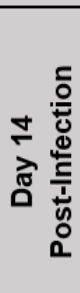 } & DPM 233 & $-0.07 \pm 0.11$ & $-0.11 \pm 0.11$ & $-0.03 \pm 0.13$ & $0.01 \pm 0.18$ \\
\hline & CB 233 & $0.41 \pm 0.20$ & $0.42 \pm 0.21$ & $0.40 \pm 0.19$ & $0.51 \pm 0.22$ \\
\hline & SIL 233 & $0.53 \pm 0.26$ & $0.60 \pm 0.28$ & $0.44 \pm 0.25$ & $0.69 \pm 0.29$ \\
\hline & DPM/SIL 233/233 & $1.40 \pm 0.58$ & $1.46 \pm 0.59^{E}$ & $1.42 \pm 0.64$ & $0.85 \pm 0.55$ \\
\hline & CB/SIL 233/233 & $2.38 \pm 0.71^{F}$ & $2.43 \pm 0.72^{F}$ & $2.31 \pm 0.71^{F}$ & $1.97 \pm 0.60^{\mathrm{G}}$ \\
\hline
\end{tabular}

All data are shown as mean fold change over control \pm standard error: ${ }^{A}$ different from control and DPM groups; ${ }^{\mathrm{B}}$ different from DPM only; ${ }^{\mathrm{E}}$ different from control only; ${ }^{\mathrm{F}}$ different from control and all single dose groups; ${ }^{\mathrm{G}}$ different from all other groups; and ${ }^{\mathrm{H}}$ different from control and SIL groups. Statistical significance is $p \leq 0.05$. 


\section{Figure 1:}

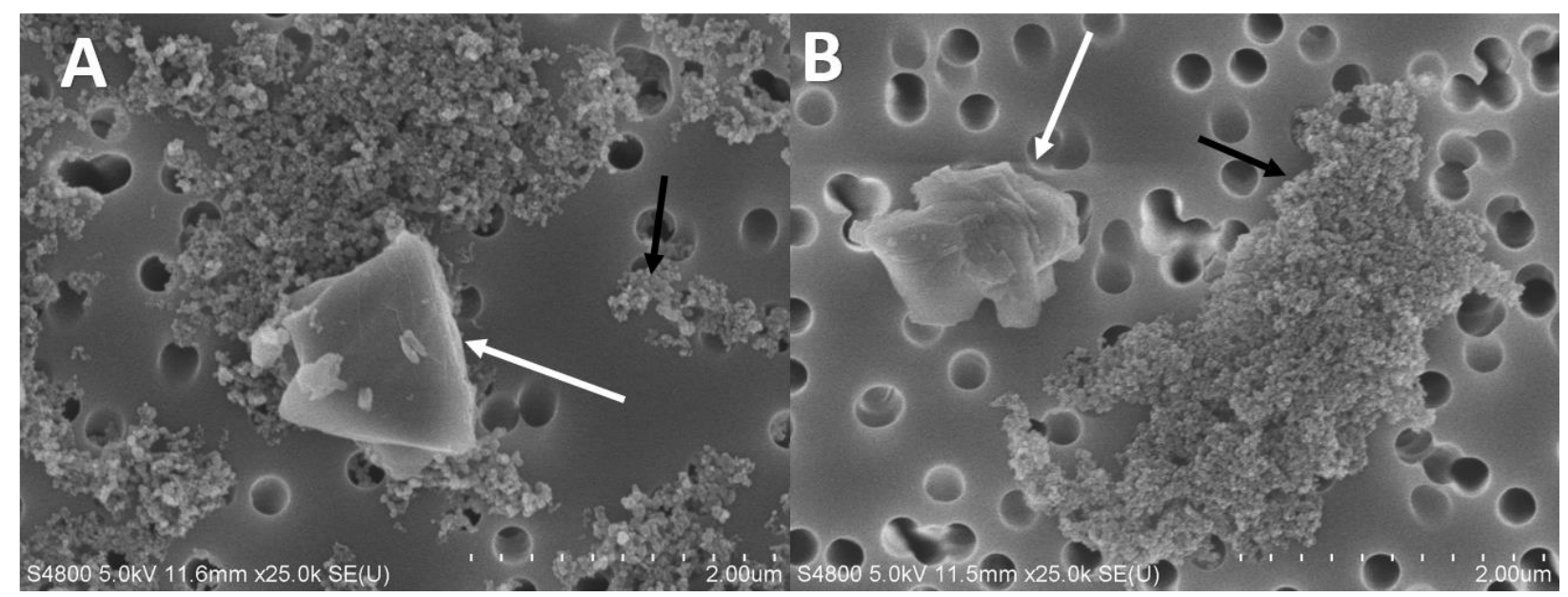

Figure 1: Field emission scanning electron microscopy (FESEM) images of particles. (A) 233 $\mu \mathrm{g}$ SIL and $233 \mu \mathrm{g}$ DPM in combination prepared in PBS solution, diluted 1:10 in PBS, then dried onto a filter under 25,000x magnification (micron bar $=2 \mu \mathrm{m}$ with 10 segments of $0.2 \mu \mathrm{m}$ each) (B) $233 \mu \mathrm{g}$ SIL and $233 \mu \mathrm{g}$ CB prepared in PBS solution, diluted 1:10 in PBS, then dried onto a filter in PBS solution under 25,000x magnification (micron bar $=2 \mu \mathrm{m}$ with 10 segments of $0.2 \mu \mathrm{m}$ each). White arrows indicate SIL particles and black arrows indicate DPM or CB agglomerates, respectively. 
Figure 2:

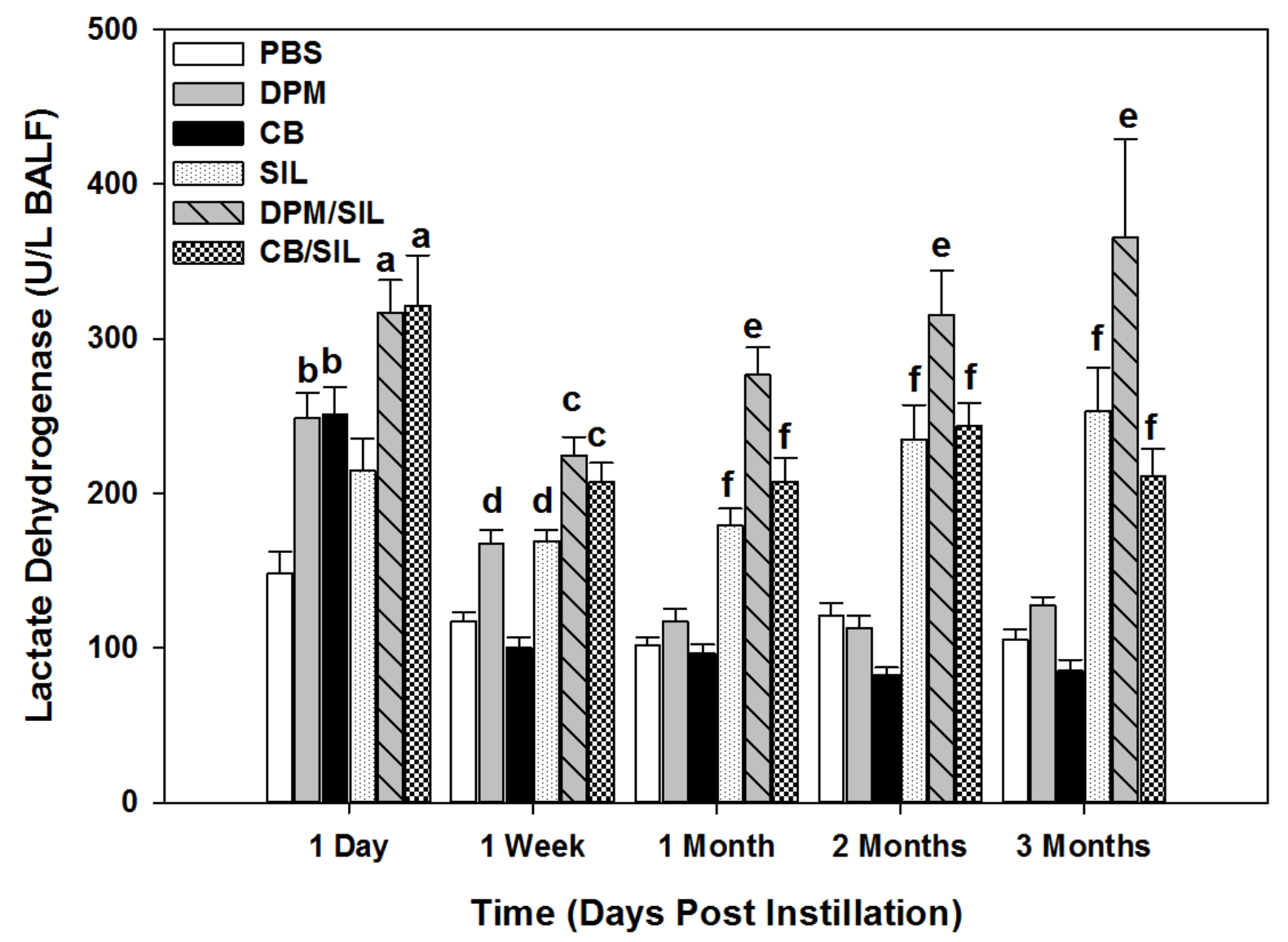

Figure 2: Lactate dehydrogenase activity (LDH) in BALF after exposure to DPM, CB, SIL, a combination of DPM and SIL (DPM/SIL), or a combination of CB and SIL (CB/SIL): ${ }^{\text {a different }}$ from control and SIL groups; ${ }^{b}$ different from control only; ${ }^{c}$ different from control and all single dose groups; ${ }^{\mathrm{d}}$ different from control and CB; ${ }^{\mathrm{e}}$ different from all other groups; and ${ }^{\mathrm{f}}$ different from control, DPM, and CB. Statistical significance is $p \leq 0.05$. 
Figure 3:

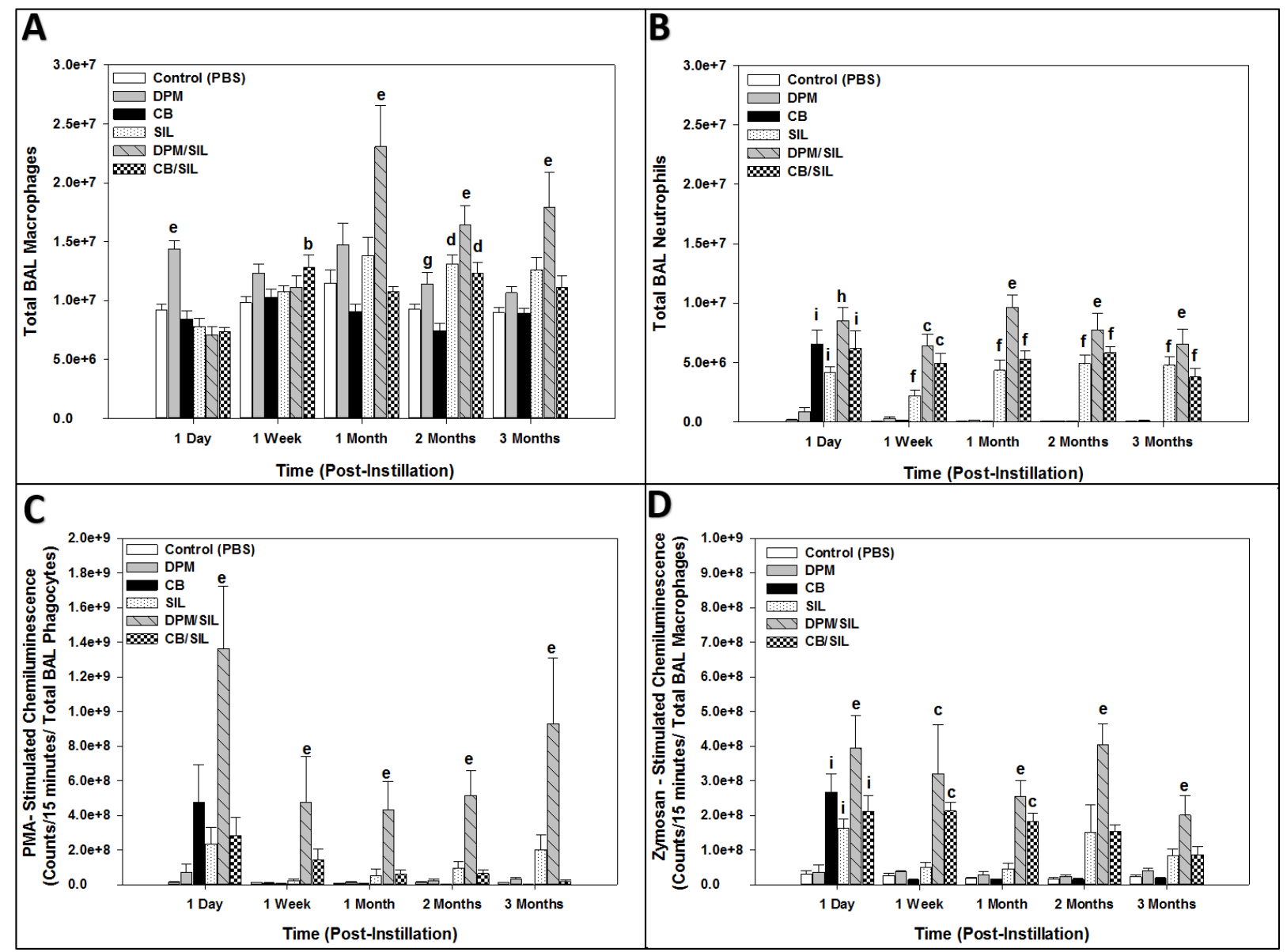

Figure 3: Total AMs (A) and neutrophils (B) in the BALF following exposure to DPM, CB, SIL, $\mathrm{DPM} / \mathrm{SIL}$, or CB/SIL. Production of oxidants by total phagocytes stimulated with PMA (C) or by AMs only stimulated by zymosan (D) measured by chemiluminescence following exposure to DPM, CB, SIL, DPM/SIL, or CB/SIL: ${ }^{b}$ different from control only; ${ }^{c}$ different from control and all single dose groups; ${ }^{d}$ different from control and $\mathrm{CB}$; ${ }^{\mathrm{e}}$ different from all other groups; ${ }^{\mathrm{f}}$ different from control, DPM, and CB; 9 different from CB only; ${ }^{\mathrm{h}}$ different from control, DPM and SIL groups; and 'different from control and DPM groups. Statistical significance measured as $p \leq$ 0.05 . 
Figure 4:

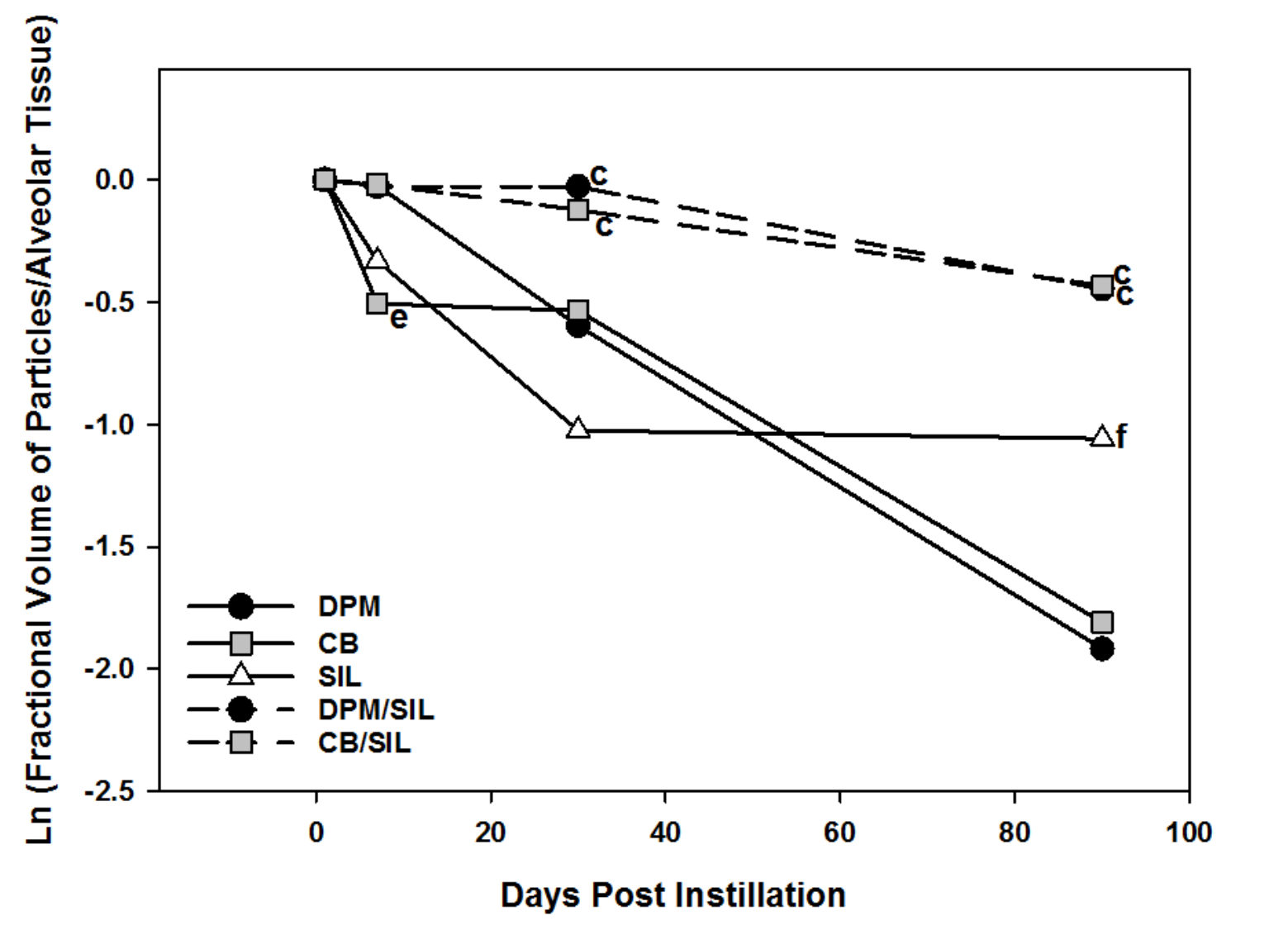

Figure 4: Particle clearance curve for each treatment group as quantitatively evaluated by morphometric analysis of tissue For each group an $n=5$ tissue sections per time-point were morphometrically assessed: ${ }^{\mathrm{c}}$ different from all single dose groups; ${ }^{\mathrm{e}}$ different from all other groups; and ${ }^{\mathrm{f}}$ different DPM and CB groups. $\mathrm{p} \leq 0.05$. 
Figure 5:

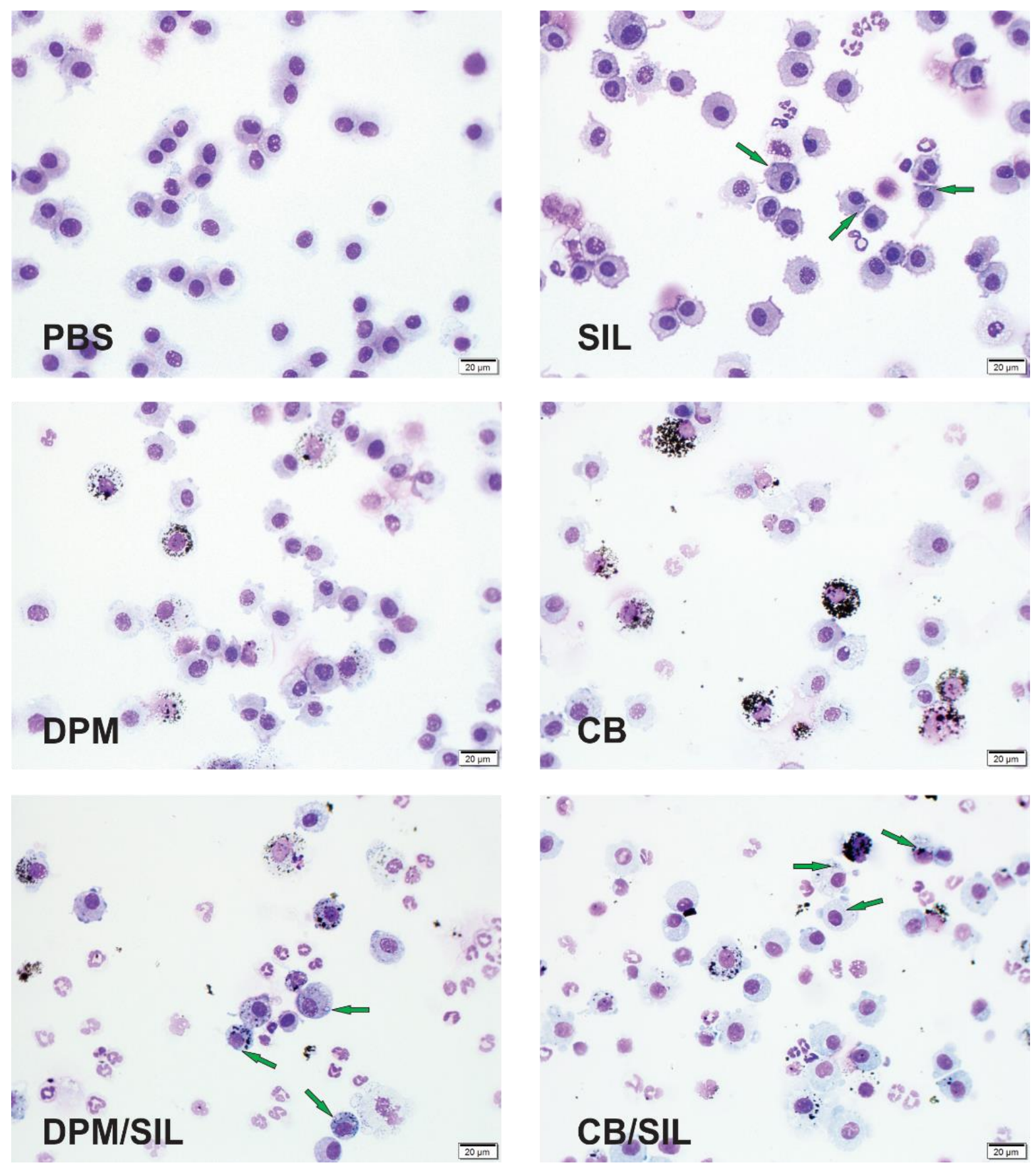

Figure 5: Light field microscopy images of cytospins used to qualitatively assess particulate clearance by BAL AMs at 1 day following instillation. Images were taken at 40x magnification and scale bar represents $20 \mu \mathrm{m}$ in length. Green arrows indicate the presence of silica particulates within macrophages. 
Figure 6:
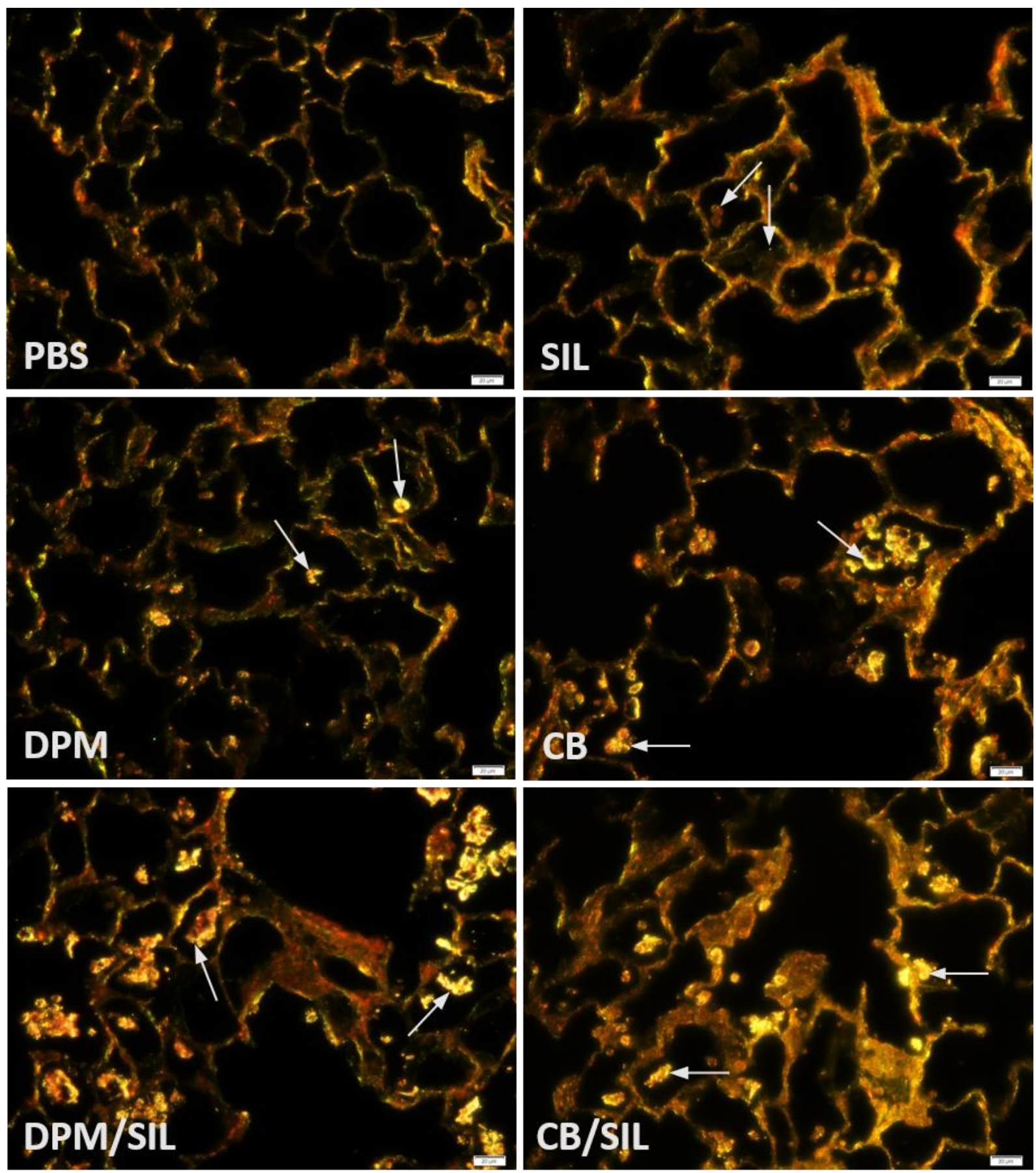

Figure 6: Dark field microscopy images of Sirius red stained tissue representing injury and particulate load in cells at 1 day following instillation. Images were taken at 20x magnification and scale bar represents $20 \mu \mathrm{m}$ in length. White arrows indicate the presence of particulate within macrophages. 
Figure 7:
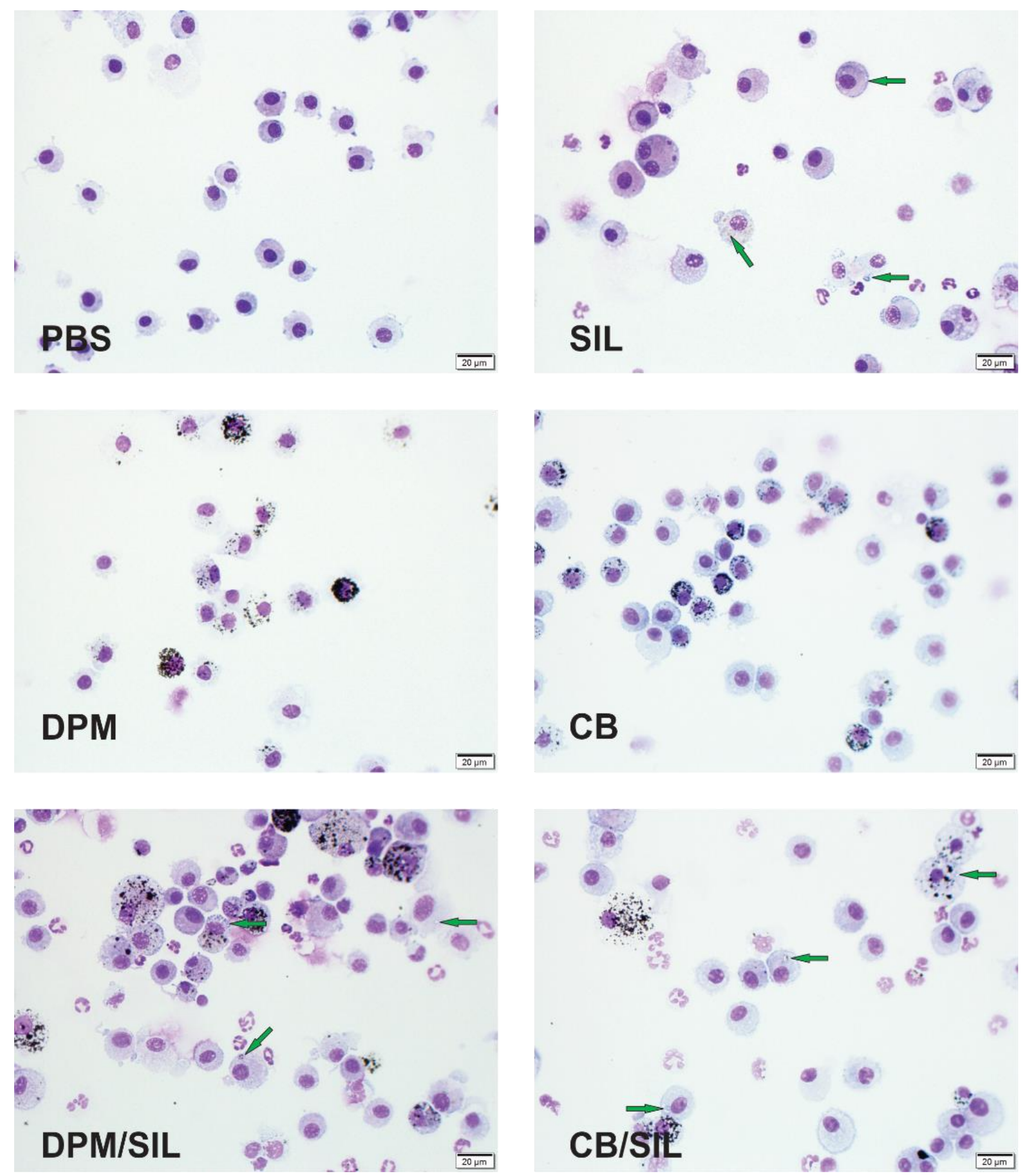

Figure 7: Light field microscopy images of cytospins used to qualitatively assess particulate clearance by BAL AMs at 1 month following instillation. Images were taken at 40x magnification and scale bar represents $20 \mu \mathrm{m}$ in length. Green arrows indicate the presence of silica particulates within macrophages. 
Figure 8:
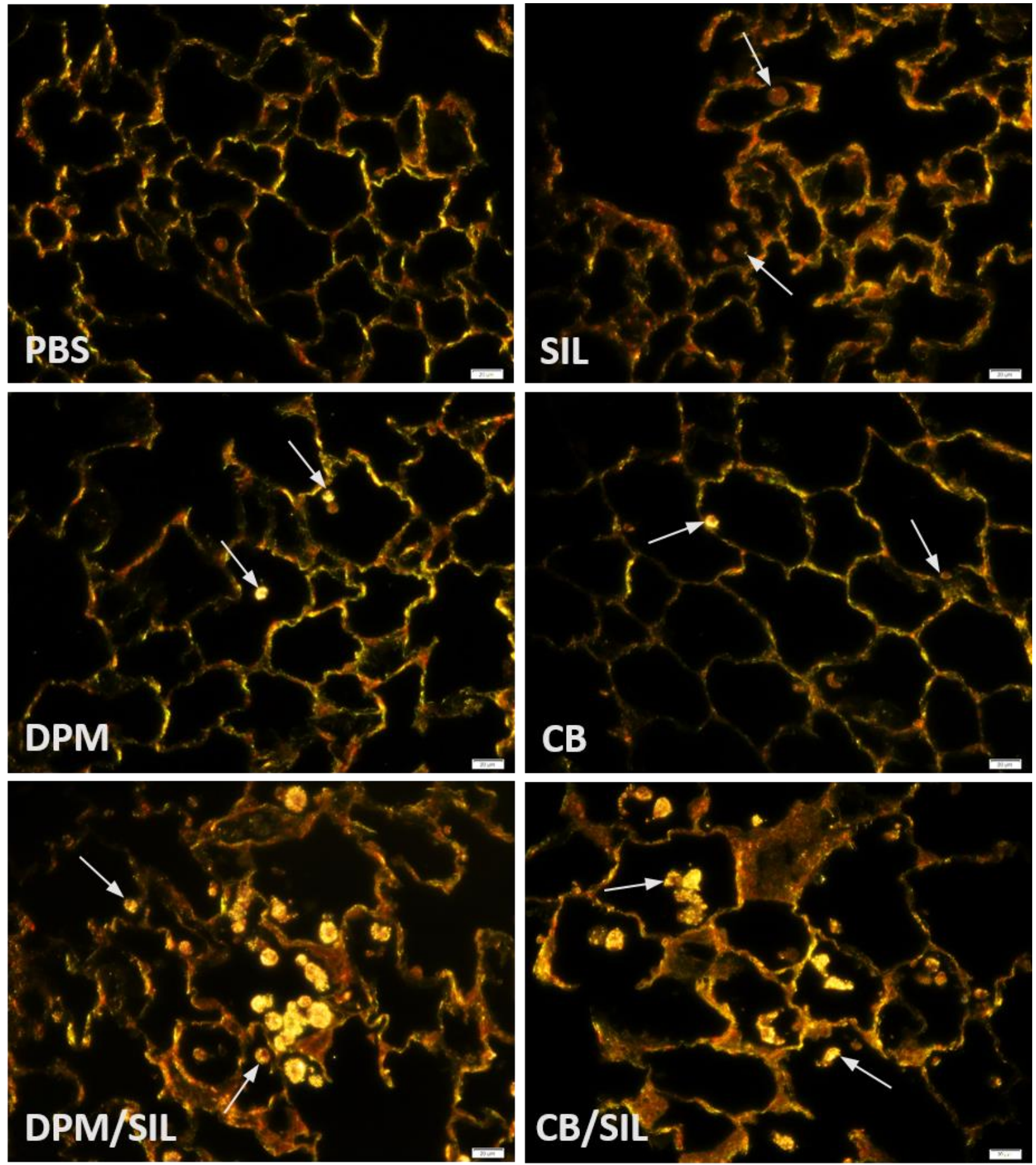

Figure 8: Dark field microscopy images of Sirius red stained tissue representing tissue injury and particulate load in cells at 1 month following instillation. Images were taken at 20x magnification and scale bar represents $20 \mu \mathrm{m}$ in length. White arrows indicate the presence of particulate within macrophages. 
Figure 9:

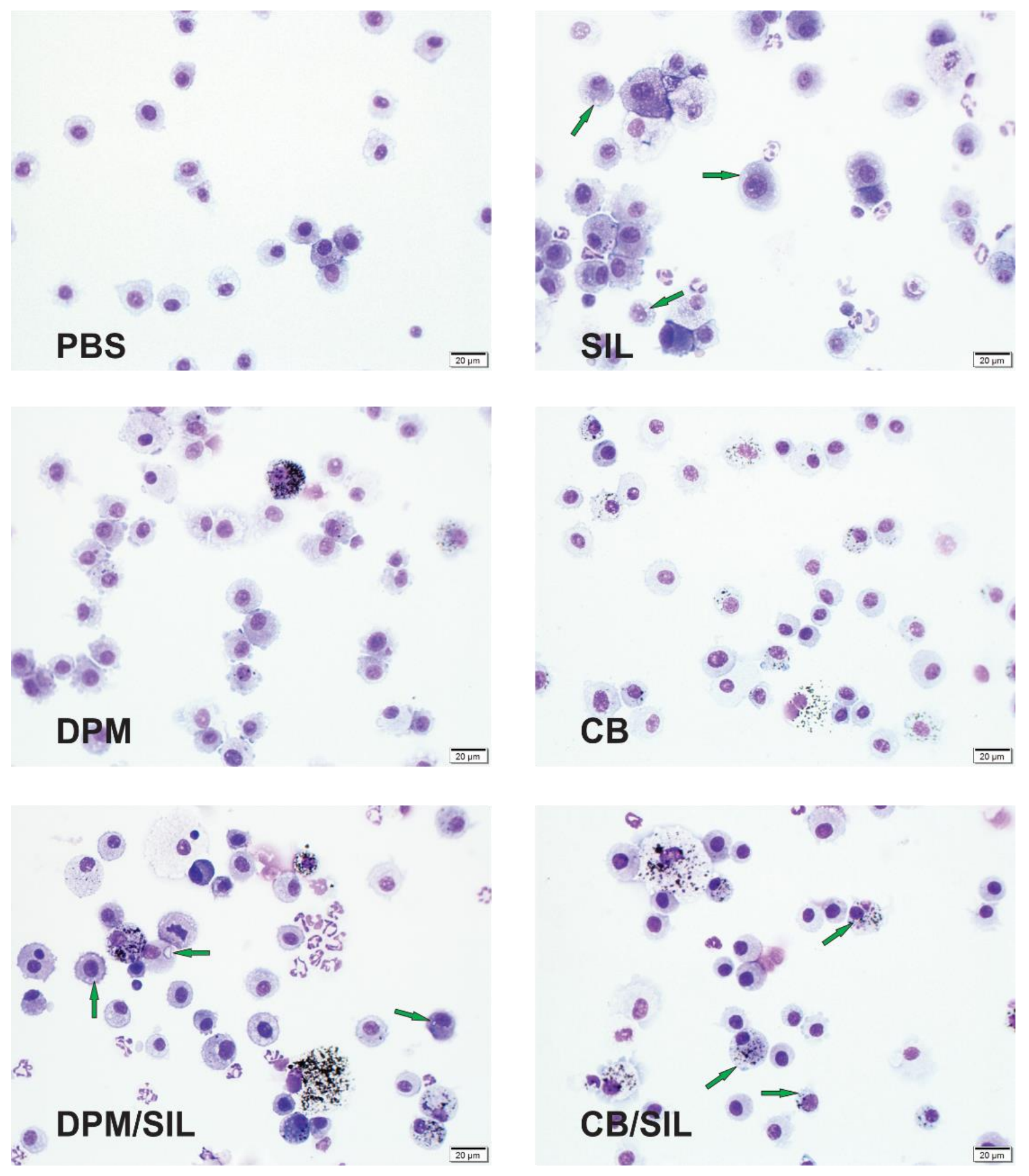

Figure 9: Light field microscopy images of cytospins used to qualitatively assess particulate clearance by BAL AMs at 3 months following instillation. Images were taken at $40 \mathrm{x}$ magnification and scale bar represents $20 \mu \mathrm{m}$ in length. Green arrows indicate the presence of silica particulates within macrophages. 
Figure 10:
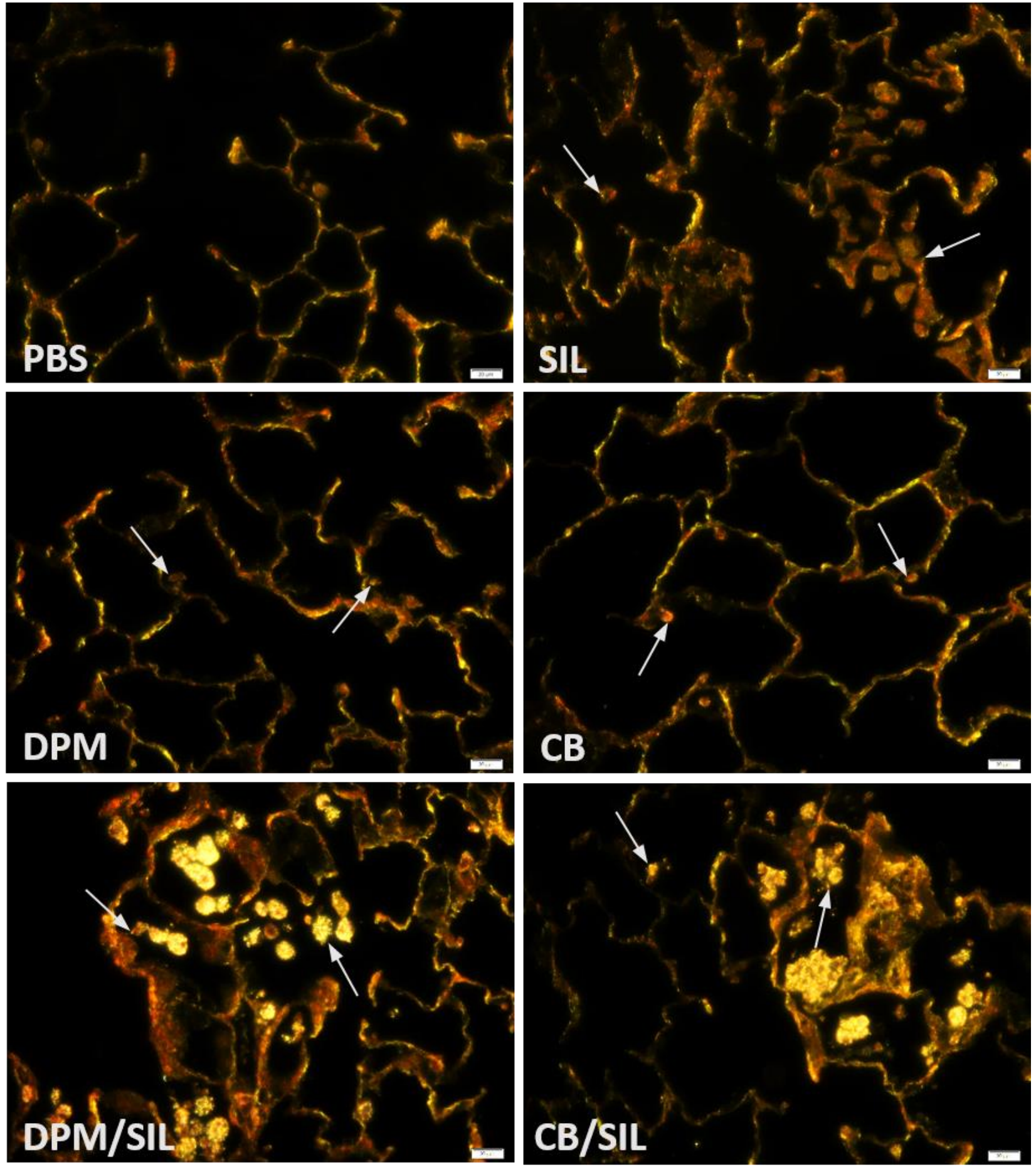

Figure 10: Dark field microscopy images of Sirius red stained tissue representing tissue injury and particulate load in cells at 3 months following instillation. Images were taken at 20x magnification and scale bar represents $20 \mu \mathrm{m}$ in length. White arrows indicate the presence of particulate within macrophages. 
Figure 11:

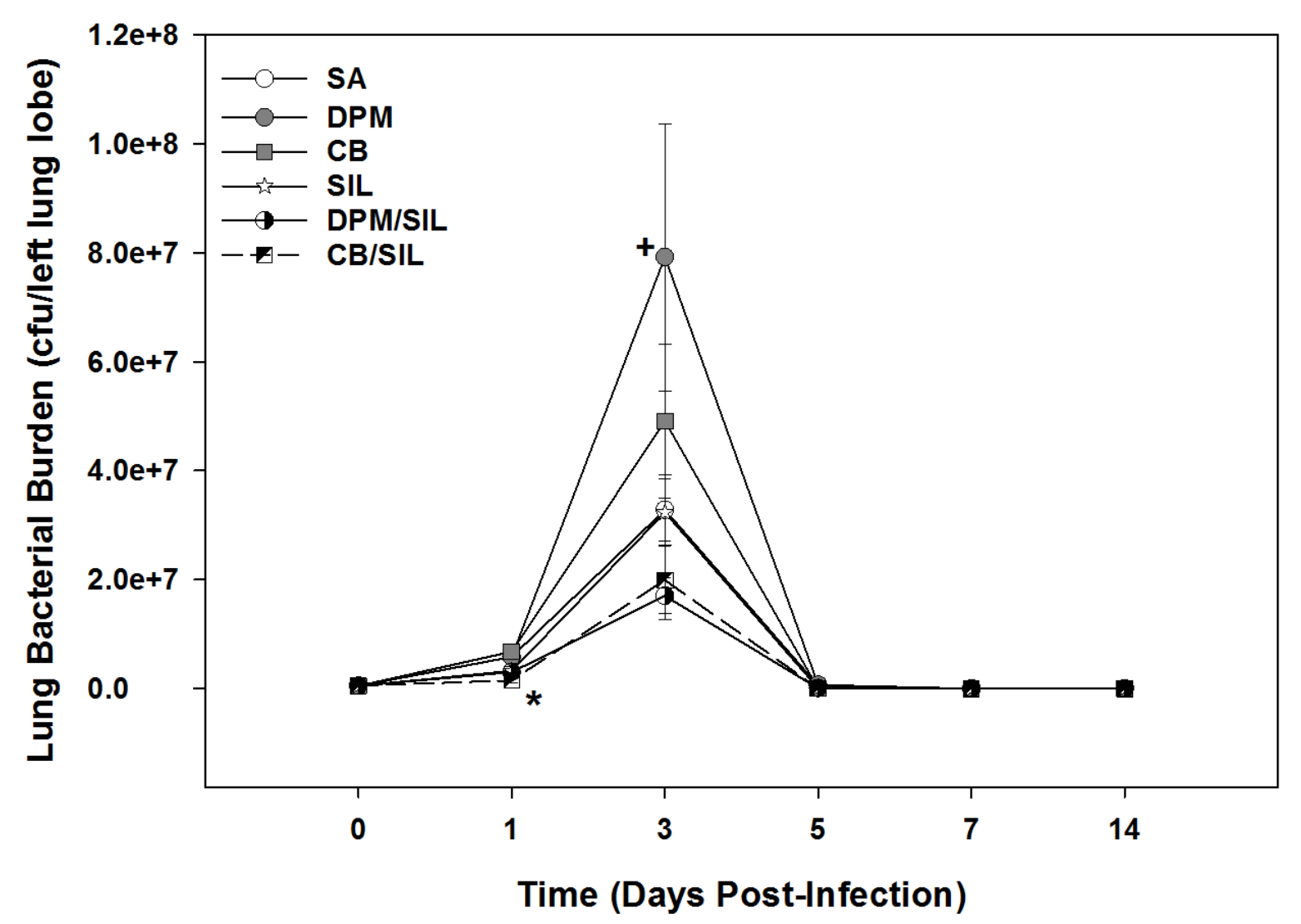

Figure 11: Lung clearance of bacteria from the left lung over the time-course recovered by lavage following exposure to DPM, CB, SIL, DPM/SIL, or CB/SIL followed by LM infection. ${ }^{*} \mathrm{CB} / \mathrm{SIL}$ lower than control and CB only. ${ }^{+} \mathrm{DPM}$ greater than control, SIL, and both combo groups. Statistical significance is $p \leq 0.05$. 
Figure 12:
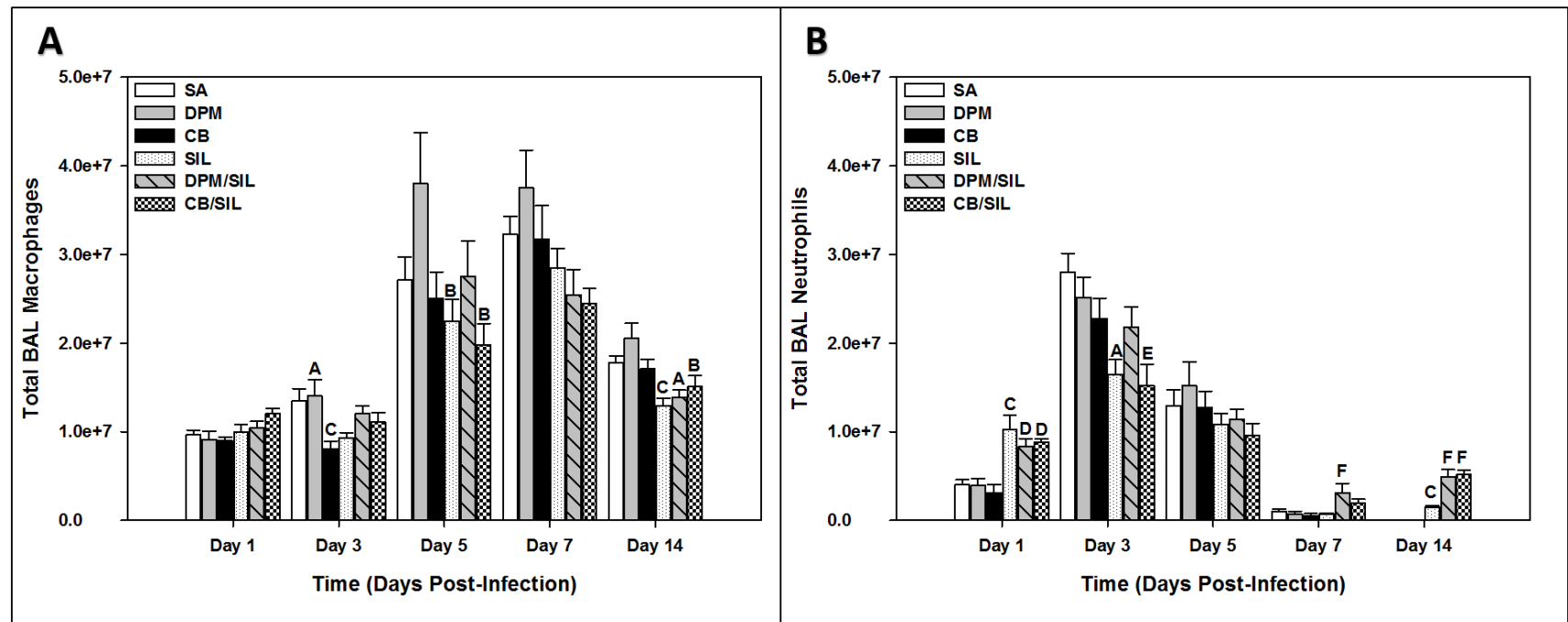

Figure 12: Total AMs (A), and total neutrophils $(B)$ recovered by lavage following exposure to DPM, CB, SIL, DPM/SIL, or CB/SIL followed by LM infection. All data are shown as mean \pm standard error: ${ }^{A}$ different from control and DPM groups; ${ }^{B}$ different from DPM only; ${ }^{C}$ different than control, DPM, and CB groups; ${ }^{\mathrm{D}}$ different from control and CB groups; ${ }^{\mathrm{E}}$ different from control only; and $\mathrm{F}$ different from control and all single dose groups. Statistical significance is $p \leq$ 0.05 . 
Figure 13:

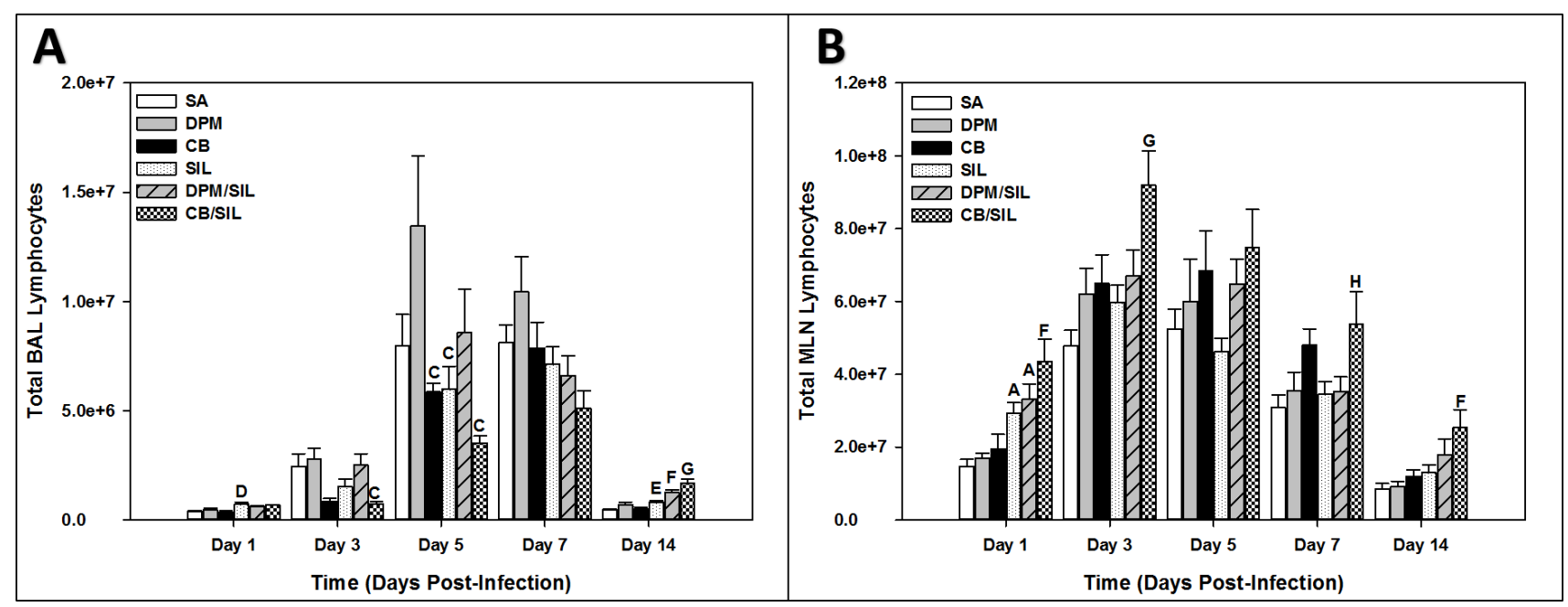

Figure 13: Total Lymphocytes recovered from BALF (A), and mediastinal lymph nodes (B) following exposure to DPM, CB, SIL, DPM/SIL, or CB/SIL followed by LM infection. All data are shown as mean \pm standard error: ${ }^{A}$ different from control and DPM groups; ${ }^{C}$ different than control, DPM, and CB groups; ${ }^{D}$ different from control and CB groups; ${ }^{\mathrm{E}}$ different from control only; ${ }^{\mathrm{F}}$ different from control and all single dose groups; ${ }^{\mathrm{G}}$ different from all other groups; and ${ }^{\mathrm{H}}$ different from control and SIL groups. Statistical significance is $p \leq 0.05$. 


\section{Chapter 4 - Study 3}

The following represents data that will be submitted to a peer-reviewed journal for publication. 
Pulmonary Toxicity following Repeated Co-exposure to Diesel Emission Particulates and a-Quartz Crystalline Silica in the Sprague-Dawley Rat

Breanne Y. Farris ${ }^{1,2}$, Robert R. Mercer ${ }^{1}$, James M. Antonini ${ }^{1,2}$, Bean T. Chen ${ }^{1}$, Diane Schwegler-Berry ${ }^{1}$, Michael L. Kashon ${ }^{1}$, Jeffrey S. Fedan ${ }^{1,2}$, and Jenny R. Roberts ${ }^{1,2}$

${ }^{1}$ National Institute for Occupational Safety and Health, Morgantown, WV ${ }^{2}$ West Virginia University, Morgantown, WV 


\section{Abstract}

The effects of repeated pulmonary co-exposures to silica and diesel particulate matter (DPM) that may occur in various occupational settings were investigated in vivo. Previous acute single dose co-exposures revealed that DPM increased the inflammatory effects of silica in the lung at time-points of up to three months. The goal of this study was to investigate the effects of subacute repeated pulmonary co-exposures to silica and DPM. Rats were exposed by intratracheal instillation (IT) to $116 \mu \mathrm{g}$ of silica (SIL), $25 \mu \mathrm{g}$ of DPM, SIL and DPM combined in phosphate-buffered saline (PBS), or to PBS alone (control) once per week for 4 weeks. At one day, one month, and three months following the last exposure bronchoalveolar lavage (BAL) and histopathology were performed, and lymphocytes from local lymph nodes were phenotyped to assess lung injury, inflammation, and immune response. SIL alone caused inflammation, oxidant production, lung injury, increased populations of immune proteins and cells in the lung, and increased lymphocyte populations in the local lymph nodes; whereas, DPM alone caused a lesser degree of injury primarily at one day post-exposure, enhanced proliferation of local lymph node cells at one month, but did not increase inflammatory cells and proteins in the lung and suppressed levels of some immunoregulatory proteins. Co-exposure to DPM and SIL resulted in neutrophilic influx and lung injury comparable to that of SIL alone. Oxidant production by stimulated macrophages in the co-exposure group was significantly elevated at all time points compared to DPM and control and at 1 day and 1 month when compared to all groups. Coexposure also significantly increased lung and local lymph node lymphocytes at all times points compared to DPM and control, but further increased T cells in the lung at one month and proliferation of total lymphocytes in the local lymph node at 3 months over SIL alone. Overall, while DPM co-exposure with SIL exhibited the capability to exacerbate oxidant production and increase lymphocyte populations to a greater degree than SIL in the lung, most parameters of toxicity were comparable to that of SIL, indicating SIL as the primary factor in toxicity. However, alteration in immune cell populations and immune proteins secreted in the lung by the presence of DPM in the co-exposure may alter overall immune response.

\section{Introduction}

Due to the vast number of industrial occupations that involve the disruption of terrain including surface and below-ground mining, tunneling, construction, road construction, and excavation, the health risks of exposure to silica have been recognized and well-characterized $[4,23]$. Furthermore, with the advent of diesel engine powered equipment in the late 1800s [5] 
and the expanding utilization in these industries for enhanced productivity, exposure of workers to respirable particulate mixtures including silica and diesel particulate matter (DPM) is a concern [279, 461]. Though toxicity of DPM and silica as individual exposures has been wellinvestigated, there is a possibility that health effects observed following a co-exposure to the particulates may be exacerbated or altered following exposure to the mixture versus the individual exposures alone.

Previously, a single acute exposure to either particle individually or as a co-exposure were investigated at various doses derived to represent acute, short duration exposures of varying degrees in industrial operations [547]. The findings from these studies demonstrated that doses of DPM that were not found to be significantly toxic individually, had the potential to exacerbate inflammation, oxidant production, alterations of innate immune response, and the incidence of the onset of fibrotic changes in the lung in a co-exposure with silica over that of silica alone. Alterations in inflammation, injury, oxidant production, and immunological response were determined to be due in part to specific characteristics of DPM, rather than an increase in overall particle load, as these changes were not observed for similar doses of carbon black, the core material of DPM, in combination with silica (Chapter 3). However, some indications of pathology, including alveolar or bronchiolar epithelial hyperplasia and fibrosis, were determined to be mainly initiated by silica and enhanced by the presence of increased particle load, as effects were similar between combinations of either DPM or CB in equal doses with silica.

Fibrotic and toxic characteristics of crystalline silica have been determined, and both acute high doses, as well as longer exposures to reduced doses, lead to progressive fibrosis, and in chronic exposure, cancer [4]. Mechanisms that govern the development of pathology are not clearly delineated; however, oxidative stress and continuous lung injury due to persistence of silica in the lung are involved. Inflammation and oxidative stress in response to exposure to DPM are also well-studied [467, 473-476]; DPM exposure is classically associated with obstructive changes including emphysema and COPD, rather than fibrosis [411, 415, 517, 518]. Our previous studies examined single exposures at doses of DPM and silica that represented various concentrations in a model of a two-week industrial exposure. This study investigated a lower but more-prolonged exposure to particles employing a sub-chronic repeated dosing schedule. The goal of this study was to determine if sub-chronic exposure to DPM further enhanced the effects of silica, or if this longer, lower dose exposure caused effects that differ from the previous studies. To address this, particles were administered to rats, individually and in a co-exposure one time per week for 4 weeks. Parameters of lung injury, inflammation, 
oxidant production, and immune response were assessed at 1 day, 1 month, and 3 months following the last exposure. The endpoints selected were similar to studies 1 and 2 for comparative purposes.

\section{Methods}

Particles. Particles in these studies are used as surrogates to represent work site exposures. DPM was obtained from the National Institute of Standards and Technology (NIST) as a commercially available NIST Standard Reference Material (SRM) 2975 (referred to as DPM in the study). SRM 2975 was originally collected from an industrial forklift in the 1990s [540]. The surrogate particle used for $\mathrm{SiO}_{2}$ was $\alpha$-quartz crystalline silica (SIL; MIN-U-SIL® 5, US SILICA; Berkeley Springs, WV).

Dose Determination. Doses were derived from field measurements of particles and applied to the equation below (EQ1) to quantify the amount of particle that would deposit in the lung of an average male worker doing moderate work for 12 hours per day for a period of 7 continuous days using a pulmonary deposition efficacy for each particle derived from a software-based algorithm that considers particle characteristics including shape and density [505]. These doses were then normalized from the surface area of human lung to the rat lung to obtain doses for in vivo studies (EQ2). There are very few studies reporting carbon levels representative of diesel emissions at above ground mining operations such as hydraulic fracturing sites. DPM in underground mining operations has been measured in excess of $500 \mu \mathrm{g} / \mathrm{m}^{3}$ as elemental carbon (EC) as reviewed by Pronk et al. (2009). In this study, the dose of DPM was derived from high EC level measurements taken during underground mining of $637 \mu \mathrm{g} / \mathrm{m}^{3}(0.637$ $\mu \mathrm{g} / \mathrm{l}$ )(EQ3) [506].The SIL dose was derived from the highest $\mathrm{SiO}_{2}$ levels measured at hydraulic fracturing site visits [486], $2.5 \mathrm{mg} / \mathrm{m}^{3}$ (2.5 $\left.\mu \mathrm{g} / \mathrm{l}\right)$ (EQ4). These levels are also common or exceeded in underground mining operations as well [548, 549].

\section{EQ1 - Basic equation for standard worker lung deposition:}

$=($ field concentration, $\mu \mathrm{g} / \mathrm{l}) \times($ minute ventilation, $\mathrm{l} / \mathrm{min}) \times($ exposure time per day, $\mathrm{min} / \mathrm{d})$ $x$ (total fracking days, $d$ ) $x$ (pulmonary deposition efficacy, unitless)

\section{EQ2 - Equation normalizing for species specific lung surface area:}

$=($ worker's deposited dose/average male worker lung surface area) $x$ (average rat lung surface area) 


\section{EQ3 - Equation for DPM dose:}

Worker lung deposition $=(0.637 \mu \mathrm{g} / \mathrm{l}) \times(20 \mathrm{I} / \mathrm{min}) \times(720 \mathrm{~min} /$ day $) \times(7$ days $) \times(0.10) \approx$ $6,421 \mu \mathrm{g}$

Dose calculated $=\left(6,421 \mu \mathrm{g} / 102.2 \mathrm{~m}^{2}\right) \times\left(0.4 \mathrm{~m}^{2}\right) \approx 25 \mu \mathrm{g}$ per rat

\section{EQ4 - Equation for SIL dose:}

Worker lung deposition $=(2.5 \mu \mathrm{g} / \mathrm{I}) \times(20 \mathrm{l} / \mathrm{min}) \times(720 \mathrm{~min} /$ day $) \times(14$ days $) \times(0.118)=$ 29,736 $\mu \mathrm{g}$ per worker

Dose calculated $=\left(29,736 \mu \mathrm{g} / 102.2 \mathrm{~m}^{2}\right) \times\left(0.4 \mathrm{~m}^{2}\right) \approx 116 \mu \mathrm{g}$ per rat

DPM and SIL Preparation and Characterization. Particles were prepared in USP-grade phosphate-buffered sterile saline (PBS) without $\mathrm{Ca}^{2+}$ or $\mathrm{Mg}^{2+}$ (AMERESCO; Solon, $\mathrm{OH}$ ) for intratracheal instillation. A sonication process was used to disperse agglomerated particles. DPM was prepared as a $2 \mathrm{mg} / \mathrm{ml}$ stock solution and SIL was prepared as a $10 \mathrm{mg} / \mathrm{ml}$ stock solution, vortex mixed for one min, followed by 1 min of continuous sonication (GE130PB ultrasonic processor; General Electric, Boston, MA). Aliquots of stock solution were then further diluted to $25 \mu \mathrm{g}$ DPM, $116 \mu \mathrm{g}$ SIL, or $25 \mu \mathrm{g}$ DPM + $116 \mu \mathrm{g}$ SIL in $0.3 \mathrm{ml}$ PBS. Particles were then vortex mixed for an additional 30 secs, followed by 15 secs of sonication prior to intratracheal instillation.

Animals. Male Sprague-Dawley [Hla:(SD) CVF] (SD) rats from Hilltop Lab Animals (Scottdale, PA), weighing approximately $300 \mathrm{~g}$ and free of viral pathogens, parasites, mycoplasmas, Helicobacter, and CAR Bacillus, were used for all studies. Rats were housed in the pathogen free, environmentally controlled, AAALAC-accredited facility. The rats were housed in ventilated polycarbonate cages, two per cage, on Alpha-Dri cellulose chips and hardwood Beta chips as bedding; they were provided HEPA-filtered air, irradiated Teklad 2918 diet, and tap water ad libitum; and were allowed to acclimate for one week before exposure. Rats were not restricted from enrichment activity (i.e., chewing/climbing). All animal procedures used during the study were reviewed and approved by the IACUC at NIOSH.

Repeated Dose Study Design. In Vivo Lung Injury, Inflammation, and Disease. On day 0, 7, 14 , and 21 rats were lightly anesthetized with an intraperitoneal injection of $30-40 \mathrm{mg} / \mathrm{kg}$ sodium methohexital (Brevital; Eli Lilly, Indianapolis, IN) and intratracheally instilled with $25 \mu \mathrm{g}$ DPM, $116 \mu \mathrm{g}$ SIL, $25 \mu \mathrm{g}$ DPM + $116 \mu \mathrm{g}$ SIL (DS), or vehicle control (PBS). All particles were delivered in $0.3 \mathrm{ml}$ USP grade PBS. All animals were humanely euthanized at 1 day, 1 month, or 3 
months following final particle exposure ( $n=8 / \mathrm{group} /$ time point) with an intraperitoneal injection of $100-300 \mathrm{mg} / \mathrm{kg}$ sodium pentobarbital followed by exsanguination. The trachea was cannulated, bronchoalveolar lavage (BAL) was performed on the right lung lobes, and BAL cells (BALCs) and fluid (BALF) were retained for analysis of parameters indicative of inflammation, injury, oxidant production, and immune function as described below. Lymphocytes from local lymph nodes draining the lung were also differentiated by phenotype. The left lung was excised, pressure-inflated with $10 \%$ neutral-buffered formalin, and weighed with water-displacement to determine fixed lung volume. Histopathological assessment of lung injury, inflammation, and disease were performed and morphometric analysis of particle clearance was conducted.

BAL. BAL was performed at 1 day, 1 month, and 3 months following the last exposure. The trachea was cannulated, the chest cavity was opened, the left lung bronchus was clamped off, and BAL was performed on the right lung lobes. BAL was recovered in two fractions As described previously [547]. For each animal, both fractions of BAL were centrifuged, the acellular fluid (supernatant) from the first fraction was retained for further analysis described for BALF below, the supernatant from the second fraction was discarded, and the cell pellets (BALC) from both fractions were combined and re-suspended in $1 \mathrm{ml}$ of PBS.

BALF Analysis: LDH Activity and Proteins: The level of LDH activity in the BALF of all treatment groups was measured at 1 day, 1 month, and 3 months following the final exposure to evaluate cytotoxicity as a measure of lung injury. Measurement of LDH activity in the acellular fluid was obtained using a Cobas Mira chemistry analyzer (Roche Diagnostic Systems; Montclair, IN). LDH activity was quantified by detection of the oxidation of lactate coupled to the reduction of $\mathrm{NAD}^{+}$at a spectrophotometric setting of $340 \mathrm{~nm}$. Cytokines and chemokines involved in inflammatory, tissue remodeling, and immune responses were measured at each time point after exposure in the BALF of rats treated with PBS, DPM, SIL, or DS combination, with commercially-available enzyme-linked immunosorbent assay (ELISA) kits or by multiplex array. The following cytokines and chemokines were quantified by ELISA: interleukin (IL)-10, IL12p70, monocyte chemotactic protein (MCP)-1, macrophage inflammatory protein (MIP)-2 (Novex, Life Technologies; Grand Island, New York); IL-2, IL-4, IL-6, interferon (IFN)-y (R\&D Systems; Minneapolis, Minnesota); and osteopontin (OPN, Enzo Life Sciences; Farmingdale, New York). Additionally, two matrix metalloproteinases (MMP), MMP-2 and MMP-9, were quantified by ELISA, as well as tissue inhibitor of metalloproteinase (TIMP)-1 (R\&D Systems) as

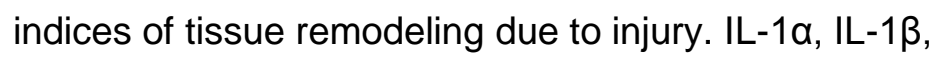


IL-5, IL-13, IL-17A, IL-18, granulocyte colony stimulating factor (G-CSF), granulocyte and macrophage colony stimulating factor (GM-CSF), eotaxin, MIP-1a, IFN-y inducible protein (IP)10, growth-regulated oncogene/keratinocyte chemoattractant (GRO/KC), lipopolysaccharide (LPS)-induced CXC chemokine (LIX), regulated on activation normal T-cell expressed and secreted (RANTES), tumor necrosis factor- $\alpha$ (TNF- $\alpha$ ), and transforming growth factor- $\beta$ (TGF- $\beta$ ) were assessed by multiplex array (Eve Technologies; Alberta, CA).

BALC Differentials. Total BALC collected from rats intratracheally-instilled with PBS, DPM, SIL, or DS were counted using a Coulter Multisizer II (Coulter Electronics; Hialeah, FL). BALC differentials were performed to determine the total number of alveolar macrophages (AMs) and neutrophils previously described (Farris et al 2017). Briefly, $5 \times 10^{4}$ cells from each rat were spun down onto slides with a Cytospin 3 centrifuge (Shandon Life Sciences International; Cheshire, England) and labeled with Hema $3 \circledR$ stain (Fisher Scientific; Kalamazoo, MI) to differentiate cell types. Two hundred cells per slide were counted, and the percentage of AMs and neutrophils was multiplied by the total number of cells to calculate the total number of each cell type.

Chemiluminescence (CL). To measure the production of reactive oxidant species by BALC, CL was measured according to the method of Antonini et al. [507] as previously described [547]. Briefly, extracellular oxidant production was measured as light emitted as a reaction of oxidants with luminol following stimulation of BAL AMs by zymosan or total BAL phagocytes (AMs and neutrophils) by phorbal myristate acetate (PMA).

\section{Phenotypic Quantification of BALC and Mediastinal Lymph Node (MLN) Cells by Flow Cytometry.}

Total BALC collected from rats were counted using a Coulter Multisizer II (Coulter Electronics; Hialeah, FL), followed by differentiation into alveolar macrophages (AMs) and neutrophils as previously described [547]. Flow cytometry was performed to enumerate and phenotype BALC. The total number of lymphocytes, B cells (CD45R), and T cells $\left(C D 3^{+}\right)$, as well as $T$ cell subsets $\left(\mathrm{CD} 4^{+} / \mathrm{CD} 8^{+}\right)$cells were quantified.

Histopathology. The left lungs of rats were fixed with $10 \%$ neutral buffered formalin by airway pressure fixation under $30 \mathrm{~cm}$-water pressure to total lung capacity for $15 \mathrm{~min}$. Lung volumes for morphometric analyses were assessed by weight of water displacement. The left lungs were embedded in paraffin, sectioned onto slides, and stained with hematoxylin and eosin (H\&E) or trichrome. An $n=5$ stained slides per treatment group per time-point were assessed; stained 
slides were analyzed for indications of inflammation, injury, and fibrosis by a certified veterinary pathologist at Charles River Laboratories (Wilmington, MA) who was blinded to the treatment groups. Indices of pathology were scored on scale of $0-5$, where $0=$ no observed effect, $1=$ minimal response, 2 = mild response, $3=$ moderate response, $4=$ marked response, and $5=$ severe response. Tissue stained lightly with Sirius red was also qualitatively examined by darkfield microscopy on an Olympus BX41 using CellSens Dimensions Software version 1.15 (Olympus, Tokyo, Japan) at a magnification of 20x in order to observe particle-loaded cells within alveolar regions and injury to alveolar septae.

Determination of Particle Clearance - Clearance was evaluated both qualitatively by examining representative images of particle loaded phagocytes from cytospins using light field microscopy on an Olympus BX63 using CellSens Dimensions Software version 1.15 (Olympus, Tokyo, Japan) and quantitatively by morphometrical analysis. Tissue sections from left lung were deparaffinized and stained lightly with Sirius Red. Slides were immersed in $0.1 \%$ picrosirius solution ( $100 \mathrm{mg}$ of Sirius Red F3BA in $100 \mathrm{ml}$ of saturated aqueous picric acid) for 2 $\mathrm{h}$ followed by washing for $1 \mathrm{~min}$ in $0.01 \mathrm{~N} \mathrm{HCl}$. Slides were then counterstained with hematoxylin for $2 \mathrm{~min}$, dehydrated, and mounted with a coverslip for imaging. Quantitative morphometric methods were used to measure the volume (\% of the alveolar wall) and enumerate particles present by standard morphometric analyses (Underwood 1970; Mercer et al. 1994). This consisted of basic point counting. An eyepiece counting overlay consisting of 11 by 11 lines (121 total points for each throw of the overlay) was used with a 40X objective. A grid pattern for throws of the counting overlay was used in order to ensure a uniform sampling of the section, which did not overweigh interior points. An $n=5$ slides was evaluated for each group at time-points of 1 day, 1 month, and 3 months following exposure. Briefly, clearance was calculated by finding the total volume of particle, normalizing data to 1 at day 1 and deriving a natural log curve from latter time-points.

Statistical Analyses. Results for all BAL parameters, and cellular phenotype were expressed as mean \pm standard error and a one-way analysis of variance (ANOVA) was performed between all treatments groups at each time point. For BAL proteins that were below the limit of detection, $1 / 2$ of the lowest limit of detection was used for statistical analysis of values. Significant differences among groups were determined using the Student-Newman-Keuls (SNK) post-hoc test using SigmaPlot for Windows Version 12.5 (Systat Software, Inc, Ekrath, Germany). For those groups failing normality or variance in the software analysis, ANOVA was run on log transformed data followed by SNK post-hoc test. For all analyses, significance was set at $p<$ 
0.05. Because data from histopathology studies are inherently categorical, nonparametric analysis of variance was assessed using SAS/STAT software, Version 9.1, of the SAS System for Windows statistical programs (SAS Institute, Inc.; Cary, NC). In this instance, groups were compared using the Wilcoxon rank sum test.

\section{Results}

Lung injury was evaluated as LDH activity in the BALF collected from rats at 1 day, 1 month, and 3 months post-final exposure to particles. LDH activity was significantly increased in all groups that contained SIL when compared to control and DPM only group (Figure 1). DPM alone also increased LDH activity compared with control at 1 day and 3 months following the last exposure. Although LDH activity was increased in the groups co-exposed to DPM and SIL, this effect was not different from SIL only at any time-point.

Protein mediators were evaluated in BALF as a measure of inflammation and immune response (Tables 1-2). DPM did not cause significant increases in the majority of the inflammatory BALF proteins that were measured when compared to control (Table 1). Only eoxtaxin was elevated at 3 months. Exposure to SIL alone caused persistent increases in the following inflammatory proteins: LIX, MIP-1 $\alpha$, MIP-2, MCP-1, IL-18, and TNF- $\alpha$. For several of the cytokines, the elevation not only persisted but increased with time. Additionally, II-1 $\beta$ and RANTES were also elevated at 3 months when compared to control. Co-exposure to DPM and SIL resulted in increased MCP-1, MIP-1 $\alpha$, MIP-2, IL-18, TNF- $\alpha$, and a trend for an increase in LIX at various time-points when compared with control and DPM. Further, combined exposure did not increase

inflammatory protein expression significantly above that of SIL, and for 3 months post-exposure, there were a number of inflammatory proteins that were significantly greater in the SIL group compared to the DS group. SIL and DS co-exposure also caused a significant increase in MMP9, a protein associated with tissue remodeling that has implications in the development of fibrotic changes in the lung, at all time-points following exposure (Table 2). Additionally, SIL exposure and DS co-exposure increased OPN at 1 day with a trend for an increase at the later time-points, and TGF $\beta 2$ at 1 and 3 months. These proteins are also integral in tissue remodeling, wound healing, and development of fibrosis. DPM alone increased OPN only at 1 day post-exposure. SIL alone caused an increase in immunoregulatory protein IL-2 at the later time points and IP-10 at all time-points (Table 2). IP-10 was also increased at the later time points in the co-exposure group. DPM alone and in the co-exposure with SIL caused a downregulation of IFN- $\gamma$. Interestingly, this effect in combination with an attenuation of several 
inflammatory cytokines relative to SIL alone indicated that DPM in a co-exposure with SIL may be exerting independent action on the immune and inflammatory response. These observations may indicate altered macrophage and lymphocyte activity in response to treatment with DPM. There were no significant changes in the following cytokines in any group: GRO/KC, G-CSF, GM-CSF, IL-1 $\alpha$, IL-4, IL-5, IL-10, IL-12p70, IL-13, IL-17A, MMP-2, and TIMP-1 (data not shown).

Cell differentials were performed on BALC to further assess inflammation (Figure 2A\&B). DPM alone did not cause an increase in the influx of AMs (Figure 2A) or neutrophils (Figure 2B). DPM did suppress macrophage influx at the one month time-point compared with control (Figure 2A). Macrophage influx was increased in all groups that contained SIL beginning at one month postexposure. Neutrophil influx significantly increased as early as one-day post-exposure in all groups containing SIL (Figure 2B), and the pattern of inflammation persisted and increased throughout the time course. There were no significant differences when comparing influx of cells between SIL and co-exposure group, which were also increased throughout the time course. BALC activation was also evaluated as oxidant production ex vivo (Figure 2 C\&D). DPM alone did not cause an increase in oxidant production by AMs and neutrophils as measured by CL. Oxidant production in the SIL group significantly increased for total phagocytes at all times postfinal exposure (Figure $2 \mathrm{C}$ ), and oxidant production by macrophages increased at later timepoints (Figure 2D). Co-exposure to DPM and SIL caused an increase in oxidant production at all time-points. Interestingly, exposure to DPM combined with SIL resulted in significantly increased oxidant production over exposure to SIL alone at one-day and one-month, suggesting a potentially higher oxidant burden in the lung of rats in the co-exposure group at these times.

Total BAL and MLN lymphocytes are shown in Figure 3 and phenotype determined by flow cytometry is shown in Tables 3 and 4. In addition to phagocytic influx described in Figure 2, exposure to SIL alone or in combination with DPM caused significantly increased lymphocyte influx into the lung as early as 1 day following exposure (Figure 3A) and proliferation in the MLN (Figure 3B) as early as one month following the last exposure. This effect was attributed primarily to $\mathrm{CD}^{+}$and $\mathrm{CD}^{+} \mathrm{T}$ cell population subsets (Tables 3 \& 4). Exposure to DPM alone caused a decrease in $\mathrm{CD}^{+}$and $\mathrm{CD}^{+}$subsets in the lung at the one-month time-point compared with control (Table 3). In the MLN, proliferation of total lymphocytes and all subsets measured were increased for DPM exposure at the same time-point. Additionally, it was noted that for the co-exposed group, DPM enhanced the influx of T cells into the lung compared with all groups, though this was only significantly different from SIL at one-month following the final exposure. 
However, in the MLN, DS exposure resulted in increased lymphocyte proliferation, particularly at later time-points compare with all groups. These results were significantly different from SIL only exposure for $\mathrm{T}$ cells as early as one-day after final exposure, and for total lymphocytes comprised primarily by increased $\mathrm{T}$ and $\mathrm{B}$ cell lymphocyte populations at three-months following exposure (Table 4).

H\&E and trichrome-stained tissue sections were evaluated by a board-certified veterinarian pathologist (Table 5). Influx of AMs, as a measure of inflammation, was increased in SIL and DS groups at later time points of one and three months following exposure when compared to control and DPM, but there was no significant change between SIL and DS. Mixed cell infiltration (MI) was initiated by SIL as there were no changes for DPM alone, and the DS group was similar to SIL for later time-points. Significant alveolar epithelial hyperplasia (AEH) and minimal to mild septal fibrosis were observed in only the combined exposure group at the later time-points, which is reflected in the sirius red stained tissue as evaluated qualitatively by dark field microscopy (Figure 5). The presence of brown granules was significantly apparent for both DPM only and DS groups as compared to control and SIL groups, indicating the presence of diesel (SIL would not appear unless examined under polarized light); interestingly, for the DPM group, brown granules were not increased at three months compared with DS group, which was still significantly elevated, indicating increased clearance in the DPM group. Macrophage particle load was qualitatively observed using images of cytospins collected from BAL that reflect particle load evaluated quantitatively by morphometry (Figure 4). This suggests that clearance of DPM in the co-exposure group may be decreased over time compared to DPM alone. Morphometrical analysis of particle clearance showed that clearance for the SIL and DS group was significantly slower than that of DPM alone at 1 day following the final exposure, and particle burden in the lung was greater for DS and SIL groups at all time points with the greatest load in the DS group (Figure 6A). Cumulative doses for each particle were also estimated based on morphometrical clearance data analyzed in study 2 . Though a degree of particle clearance may have occurred between each dosing week, assuming a constant rate of clearance throughout the repeated exposure time course of 21 days, total burden of particle at day 1 post final-exposure is still estimated to be $80 \%$ of cumulative DPM dose; whereas, $90 \%$ of either SIL or $95 \%$ of DS total dose remained at day 1 following final exposure. Additionally, rate of clearance slowed for the co-exposure group by 1 month becoming significantly slower than both SIL and DPM. At 1 day post-final exposure, DPM comprised $30 \%$ of overall remaining DS dose; however, by 3 months following exposure, approximately $90 \%$ of DS dose remaining was comprised of silica and only $10 \%$ remaining was 
comprised of DPM (Figure 6B). By three-months only 33\% of particle had been cleared in the co-exposure as compared with SIL or DPM, where $56 \%$ and $74 \%$ had been cleared, respectively (Figure 6A). Although clearance of DPM in the co-exposure is slower than DPM alone, clearance of this particle in a co-exposure is still occurring at a steady rate.

\section{Discussion}

The toxicological and fibrotic properties of crystalline silica are well-established, and both acute bolus doses of silica and more chronic lower dose exposure lead to fibrotic conditions and in the latter case, cancer [4], though the overall mechanisms of action are not fully elucidated. DPM-induced inflammation and oxidative stress are also well-investigated [467, 473-476]; though, DPM exposure is more closely associated with obstructive respiratory conditions and cancer, rather than restrictive fibrosis $[411,415,517,518]$. The current study was conducted in order to investigate a model of repeated administrations of DPM and crystalline silica, which have been previously shown to cause respiratory health effects individually, and that may be encountered in a co-exposure during different mining operations. Previous studies in our lab established that combinations of these particles, given in one bolus dose, exacerbated or altered toxicological effects observed for either particle alone. These results were particularly significant for inflammation, production of oxidants by phagocytes, initiation of fibrotic changes in the lung, and differential alteration of immune protein secretion (Farris et al 2017). Further, it was established that particle load and SIL particle exposure were the primary determinants for histopathological effects related to tissue remodeling; whereas, DPM itself caused particle specific effects related to inflammation and oxidant production when in combination with SIL in a single dose scenario (Study 2). In both studies, irrespective of the DPM doses, SIL was the primary particle by which toxic outcomes were determined, and DPM produced particle specific effects that modified or enhanced the toxicity of SIL alone, even at non-toxic doses of DPM. In this study, particles were administered in repeated lower doses in order to characterize toxicity of SIL and DPM in combination in a sub-chronic exposure scenario.

In the present study, most of the effects observed in the co-exposure correlated closely to effects that were initiated by the presence of SIL, as the SIL and DS groups did not differ significantly from each other in many instances. For the parameters of cellular injury and pulmonary inflammation, measured by the activity of LDH in the BALF and influx of macrophages and neutrophils into the lung, respectively, there were no significant differences between the SIL exposed group and the group exposed to the combination of DPM and SIL 
(Figure 1 and 2 A,B). However, when assessing the potential contribution of inflammation caused by production of oxidants by phagocytes, there was an increase in the macrophage oxidant production in the co-exposed group compared to SIL alone at one month following the final exposure. By the three-month time-point, although still increased, there were no significant differences between the two groups. Interestingly, at one-month following exposure, not only did DPM alone not cause any change in production of oxidants from that of the control group, DPM exposure alone caused a significant decrease in the influx of macrophages into the lung (Figure 2A). This effect demonstrates a distinct alteration in the toxicological responses in the lung to a combination of particles compared to single particle exposure to either DPM or SIL.

Other studies examining longer duration or higher doses of silica and DPM have also shown differential effects on the function of alveolar macrophages, which may play a role in differences in effect overall on the immune system and have been reviewed in depth $[5,115$, $127,128,511,550-552]$. While crystalline silica characteristically causes acute increases in activity of macrophages, inducing production of oxidants and triggering a cascade of inflammatory signaling events, it has also been shown to cause eventual cytotoxicity to macrophages as reviewed by Fubini and Hubbard [130]. The exact biochemical mechanism(s) that govern this event are not fully understood but apoptosis is likely triggered by the involvement of scavenger receptors and recognition of damage signals, production of oxidants, or some combination of the two events [553]. DPM, similarly, is associated with variable effects on alveolar macrophages in some instances triggering inflammatory responses by increased oxidant and cytokine production, classically associated with the development of allergic hypersensitivity; however, DPM has also been shown by several studies to suppress the activities of macrophages particularly associated with effective clearance of pathogens from the lung. Dose of DPM administered, as well as, composition of DPM are suspected to be major factors in effects upon macrophages as reviewed by Lawal [554]. With regard to effects on the immune system, silica has been shown to have variable effects dependent upon length of exposure. Acutely, silica increases inflammation and production of oxidants by phagocytes that contribute to enhanced clearance of infection acutely [113]. However, when silica exposure occurs subchronically and chronically, cytotoxicity, oxidant injury, and a possible shift in resident AM population, contribute to reduction of clearance of infection $[59,62,110]$. DPM has been shown to suppress activation and effector functions of macrophages and T cells, and these alterations are associated with increased susceptibility to infection and reduced pulmonary clearance of pathogens $[316,324,535,555,556]$. 
In the current study, when evaluating the immunological proteins that were secreted into the BALF, SIL increased the expression of many inflammatory chemokines and cytokines associated with the recruitment of immune cells to the lung including LIX, MIP-1a, MIP-2, and MCP-1 (Table 1). At day 1, expression of several of these proteins was slightly reduced in the DPM exposed group compared to control, although not to a statistically significant degree. This trend may contribute to the reduction of macrophage recruitment observed in the lung at one month following the last exposure (Figure 2A). Interestingly, these same proteins in the DS group, although increased overall, were lower when compared to SIL in some instances, and more significantly at later time-points. This suggests that DPM may have differentially altered the response to SIL in a co-exposure. At some time-points, SIL also enhanced the expression of inflammatory proteins such as TNF- $\alpha$ and IL-18 or IL-1 $\beta$ involved in the activation of inflammatory cascades including NF-KB and the inflammasome, respectively, and that are also involved in apoptotic pathways. These effects, while often mirrored in the co-exposure, were not altered by the presence of DPM suggesting that for the expression of these proteins, SIL appeared to be the initiating factor, and that DPM did not alter or further enhance the response. Proteins involved in immunoregulation and the activation/differentiation of lymphocytes and macrophages were also increased in the SIL group: IL-2, IFN-y, and IP-10 (Table 2). Similar to chemotactic factors that were down-regulated by DPM, immunoregulatory proteins were also lower than control, particularly at early time-points. These proteins were also reduced in expression in the co-exposure group as compared with SIL alone at various time-points. IP-10 expression is dependent upon the increased levels of IFN- $\gamma$, and IFN- $\gamma$ in turn activates macrophages. Therefore, it is plausible that the reduction in IFN- $\gamma$ in the DPM group at one day following exposure may contribute to reduction in macrophage recruitment and a decrease in expression of IP-10. Though expression of IFN-y is reduced for the co-exposure group throughout the time-course, IP-10 expression is only reduced at 1 day following the final exposure, and macrophage recruitment was not altered in the co-exposure group when compared to SIL alone.

The lack of the DPM-specific enhancement of various inflammatory responses, as observed in the first two studies, and a decrease in differences observed between SIL and DS groups may also be partly due to differential clearance of SIL versus DPM over time in the coexposure group. Clearance analysis in the DS group revealed that DPM accounted for only $20 \%$ of the total particle remaining at the 1 month and only approximately $10 \%$ by the 3 month timepoint. Therefore, it is likely that the persistence of SIL in the co-exposure group is the primary particle contributing to the response at the later time points; whereas in study 2 , an acute bolus 
dose higher than that in the present study may be contributing to a greater degree of particle specific effects by DPM in that study.

Another difference between the present study and studies 1 and 2 occurred in regard to lymphocyte proliferation. SIL exposure caused an increased influx of lymphocytes into the lung (Table 3). This was followed by enhanced proliferation of lymphocytes in local lymph nodes by one month following the last exposure (Table 4). These lymphocytes were found to be comprised primarily of T cells, and more specifically, the CD4 ${ }^{+}$subset of T lymphocytes. DPM caused a transient decrease in the subsets of T cells present in the lungs at one month following exposure (Table 3), which may reflect the reduction in protein expression and macrophage activity observed at this time point as discussed above (Table 1,2 and Figure 2A). For most lung lymphocyte parameters, there were no significant differences between the SIL and DS groups. However, for T cells, and more specifically $\mathrm{CD} 4^{+}$and $\mathrm{CD} 8^{+}$subsets, there were elevations in the co-exposure group as compared with SIL suggesting an increase, albeit transient, in the immunological response for this combined exposure (Table 3). Interestingly, at the same time-point, DPM alone reduced overall influx of these subsets into the lung. It is possible that load of particle is a contributing factor for this effect in the DS group accounting for the increase versus the suppressive effects of DPM alone. Similarly, Yin et al. reported that though DPM suppressed some parameters of innate immune function, recruitment of $T$ cells following an LM infection, specifically in $\mathrm{CD}^{+}$and $\mathrm{CD} 8^{+}$subsets, was sufficient for clearing bacteria despite hindered innate immunity [317].

Similar to the previous studies (study 1 and 2), the toxic properties of SIL and particle load are also the primary factors in the tissue remodeling and fibrotic effects following exposure. SIL increased the expression of tissue remodeling proteins involved in would healing and fibrosis: MMP-9, OPN, and TGFß2 (Table 2). Changes for OPN were more significant at the earliest time-point. MMP-9 was significantly increased throughout the time course, and TGF $\beta 2$ levels continued to increase over time being significantly different from DPM and control at 3 months. A similar pattern was observed for the DS group with the exception of a significant increase in TGF $\beta 2$ at 1 month in the DS group when compared to all groups. The data suggest that SIL was the primary particle causing alterations in the signaling for tissue remodeling. Interestingly, the most persistent histopathological changes related to inflammation, epithelial hyperplasia, and fibrosis occurred in the DS group. In this group, total particle, and SIL specifically, was cleared at a slower rate from the lung when compared to the individual particles, resulting in a greater total particle load over time as compared to all other groups 
(Figure 6A). Therefore, a combination of these effects may account for the increase in fibrosis observed in the DS group only.

Overall, in the previous study (Study 2), effects of the single bolus dose related to inflammatory influx of cells, increased inflammatory proteins, altered immune regulatory proteins, and increased oxidant production were particle specific responses to DPM, whereas tissue remodeling correlated better to total load of particle and the presence of SIL in the exposure. There are some differences and similarities between the acute dose and repeated subacute dose studies. The DPM specific effect of enhanced oxidant production by macrophages in the lung was conserved. However, exacerbation of lung injury indicated by LDH in BALF was not observed in the co-exposure group in the current study, where SIL and DS did not differ from each other. This could be due to a greater burden of SIL in the lungs following the repeated exposure, whereby total SIL is the primary factor for this parameter. Additionally, alterations in immunoregulatory proteins in the DPM group and in the co-exposure were more pronounced than the previous study, and a decrease in T cells in the lung was also present in the DPM group, possibly reflecting a greater effect of exposure to DPM over time versus a higher single dose exposure to DPM. In the DS group, although SIL was the primary factor causing an increase in all parameters, the effect of the subacute exposure to DPM may have contributed to some trends in slight reductions in certain parameters of inflammation and immune response in the co-exposure group relative to SIL alone.

\section{Conclusion}

The data taken together show that DPM exposure at doses in the sub-chronic exposure do not elicit significant pulmonary toxicity; however, in combination with silica, the co-exposure had the capability to alter some silica-induced pulmonary effects, primarily oxidant production, and initiation of fibrotic changes in the lung. When administered in combination with SIL, DPM had the capacity to exhibit independent action on the expression of some proteins that may alter the inflammatory/immunological response in the lung, as demonstrated by a reduction in macrophage recruitment at 1 month post-exposure and reductions in immune regulatory proteins, particularly IFN- $\gamma$. While it was not examined in this study, these results may have implications for adverse outcomes involving the immune system such as susceptibility to infection or regulation of auto-immune responses following a sub-acute or even chronic exposure to low doses of particles. Overall, the data suggest that there is an increased risk of adverse outcomes when there is a co-exposure to SIL and DPM, even at relatively non-toxic 
doses of DPM. Since clearance of particles in the co-exposure is significantly reduced, more chronic exposure may lead to an increase in severity of effects when compared to exposure to either particle alone, particularly if accumulation of particles were to exceed rate of clearance. Furthermore, the intratracheal bolus doses of particles administered in this and the previous studies (Studies 1 and 2) are much lower than what have been previously investigated in similar models [271, 325, 498, 557, 558], and better reflect deposition that would occur overtime in inhalation models. This suggests that chronic lower-level exposures to a mixture of silica and diesel exhaust in the work place may still be sufficient for the development of adverse pulmonary health effects. 
Table 1: BALF Inflammatory Proteins

\begin{tabular}{|c|c|c|c|c|c|c|c|c|c|c|}
\hline \multicolumn{11}{|c|}{ Inflammatory Chemokines/Cytokines } \\
\hline \multirow{5}{*}{ 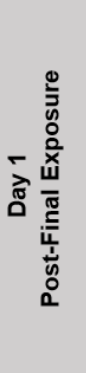 } & $\begin{array}{c}\text { Exposure } \\
\text { Group }\end{array}$ & LIX & MCP-1 & MIP-1 $1 \alpha$ & MIP-2 & Eotaxin & RANTES & IL-1 $\beta$ & IL-18 & TNF- $\alpha$ \\
\hline & CONTROL & $43.71 \pm 17.11$ & $6.23 \pm 2.57$ & $3.11 \pm 0.94$ & $396.32 \pm 25.45$ & $2.87 \pm 0.54$ & $1.29 \pm 0.14$ & $7.64 \pm 2.32$ & $670.46 \pm 184.29$ & $0.53 \pm 0.12$ \\
\hline & DPM & $20.38 \pm 3.16$ & $4.42 \pm 1.55$ & $2.33 \pm 0.53$ & $365.48 \pm 17.26$ & $1.83 \pm 0.33$ & $1.26 \pm 0.09$ & $7.41 \pm 0.77$ & $493.04 \pm 65.82$ & $0.88 \pm 0.19$ \\
\hline & SIL & $169.03 \pm 54.56^{c}$ & $32.10 \pm 8.03$ & $19.36 \pm 4.69^{\mathrm{a}}$ & $650.98 \pm 58.40^{\mathrm{a}}$ & $2.33 \pm 0.56$ & $1.19 \pm 0.15$ & $9.04 \pm 2.04$ & $931.81 \pm 139.68$ & $2.19 \pm 0.28^{\mathrm{a}}$ \\
\hline & DS & $50.08 \pm 18.70$ & $11.92 \pm 4.97$ & $12.80 \pm 1.05^{\mathrm{a}}$ & $546.40 \pm 58.74^{a}$ & $1.68 \pm 0.52$ & $1.52 \pm 0.24$ & $4.93 \pm 0.96$ & $593.20 \pm 65.33$ & $1.47 \pm 0.21^{\mathrm{a}}$ \\
\hline \multirow{4}{*}{ 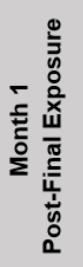 } & CONTROL & $34.37 \pm 6.52$ & $7.45 \pm 2.64$ & $3.22 \pm 0.80$ & $447.86 \pm 41.84$ & $2.27 \pm 0.52$ & $1.34 \pm 0.25$ & $6.42 \pm 1.41$ & $482.17 \pm 81.55$ & $0.62 \pm 0.12$ \\
\hline & DPM & $56.77 \pm 13.06$ & $7.45 \pm 2.71$ & $2.01 \pm 0.73$ & $436.62 \pm 29.65$ & $2.36 \pm 0.38$ & $1.30 \pm 0.02$ & $6.13 \pm 1.18$ & $384.90 \pm 58.13$ & $0.66 \pm 0.08$ \\
\hline & SIL & $253.23 \pm 111.79^{b}$ & $127.73 \pm 29.17^{a}$ & $25.37 \pm 3.96^{a}$ & $653.93 \pm 46.99$ & $2.49 \pm 0.54$ & $1.48 \pm 0.15$ & $7.18 \pm 1.25$ & $975.38 \pm 84.59^{a}$ & $1.37 \pm 0.20^{\mathrm{a}}$ \\
\hline & DS & $173.10 \pm 57.56^{b}$ & $173.82 \pm 35.44^{a}$ & $22.89 \pm 1.47^{a}$ & $409.18 \pm 108.51$ & $2.53 \pm 0.61$ & $1.96 \pm 0.17$ & $5.95 \pm 1.01$ & $989.06 \pm 50.48^{a}$ & $1.47 \pm 0.24^{a}$ \\
\hline \multirow{4}{*}{ 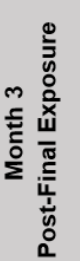 } & CONTROL & $59.09 \pm 16.86$ & $16.08 \pm 5.57$ & $4.36 \pm 1.16$ & $600.40 \pm 76.79$ & $1.27 \pm 0.86$ & $1.66 \pm 0.36$ & $4.26 \pm 0.54$ & $461.77 \pm 44.02$ & $1.08 \pm 0.14^{d}$ \\
\hline & DPM & $71.74 \pm 31.43$ & $12.05 \pm 1.38$ & $5.21 \pm 0.52$ & $597.20 \pm 60.97$ & $3.69 \pm 0.51^{\mathrm{b}}$ & $1.47 \pm 0.28$ & $9.47 \pm 2.24$ & $554.13 \pm 127.10$ & $0.55 \pm 0.17$ \\
\hline & SIL & $1428.35 \pm 276.53^{c}$ & $490.32 \pm 101.14^{a}$ & $122.38 \pm 36.30^{c}$ & $867.70 \pm 34.15^{c}$ & $1.76 \pm 0.32$ & $6.92 \pm 1.59^{c}$ & $11.10 \pm 1.66^{b}$ & $1611.68 \pm 234.49^{a}$ & $1.96 \pm 0.30^{\mathrm{d}}$ \\
\hline & DS & $435.66 \pm 237.22$ & $266.66 \pm 79.40^{\mathrm{a}}$ & $32.50 \pm 9.02^{\mathrm{a}}$ & $658.59 \pm 51.55$ & $2.45 \pm 0.53$ & $2.76 \pm 0.86$ & $6.60 \pm 0.82$ & $1061.37 \pm 145.48^{\mathrm{a}}$ & $1.25 \pm 0.20^{d}$ \\
\hline
\end{tabular}

All data are shown as mean \pm standard error: ${ }^{\mathbf{a}}$ different from control and DPM; ${ }^{\mathbf{b}}$ different from control; ${ }^{\mathbf{c}}$ different from all other groups; ${ }^{d}$ different from DPM; and ${ }^{\mathrm{e}}$ different from SIL; $p \leq 0.05$. 
Table 2: BALF Immunoregulatory Proteins and Tissue Remodeling Proteins

\begin{tabular}{|c|c|c|c|c|c|c|c|}
\hline \multicolumn{5}{|c|}{ Immunoregulatory Cytokines } & \multicolumn{3}{|c|}{ Tissue Remodeling and Wound Healing Proteins } \\
\hline \multirow{5}{*}{ 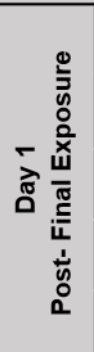 } & $\begin{array}{c}\text { Exposure } \\
\text { Group }\end{array}$ & IL-2 & IFN-Y & IP-10 & MMP-9 & OPN & TGF- $\beta 2$ \\
\hline & CONTROL & $8.49 \pm 4.65$ & $16.95 \pm 3.76$ & $13.02 \pm 2.89$ & $0.49 \pm 0.27$ & $2.50 \pm 0.84$ & $1203.69 \pm 253.78$ \\
\hline & DPM & $3.29 \pm 1.80$ & $2.44 \pm 1.09^{f}$ & $14.47 \pm 3.027$ & $0.62 \pm 0.13$ & $4.63 \pm 0.89^{b}$ & $1000.54 \pm 122.13$ \\
\hline & SIL & $2.41 \pm 2.39$ & $23.56 \pm 4.25$ & $31.97 \pm 7.99^{\mathrm{a}}$ & $2.88 \pm 0.90^{\mathrm{b}}$ & $6.62 \pm 0.88^{b}$ & $1180.39 \pm 201.22$ \\
\hline & DS & ND & $2.18 \pm 1.57^{f}$ & $24.41 \pm 2.87$ & $1.23 \pm 0.21^{b}$ & $7.37 \pm 1.06^{\mathrm{b}}$ & $902.89 \pm 111.50$ \\
\hline \multirow{4}{*}{ 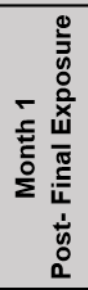 } & CONTROL & $0.32 \pm 0.31$ & $27.95 \pm 6.07$ & $12.87 \pm 3.98$ & $0.12 \pm 0.04$ & $4.91 \pm 1.15$ & $1063.19 \pm 213.98$ \\
\hline & DPM & $4.67 \pm 4.66$ & $16.10 \pm 4.62$ & $9.38 \pm 1.28$ & $0.23 \pm 0.14$ & $3.34 \pm 1.45$ & $1071.97 \pm 218.75$ \\
\hline & SIL & $19.98 \pm 4.46^{\mathrm{c}}$ & $18.72 \pm 6.49$ & $57.96 \pm 13.23^{a}$ & $3.59 \pm 0.74^{\mathrm{a}}$ & $13.27 \pm 4.73$ & $1700.34 \pm 281.85$ \\
\hline & DS & $7.39 \pm 5.00$ & $4.05 \pm 2.11^{c}$ & $66.53 \pm 5.71^{a}$ & $3.27 \pm 0.35^{\mathrm{a}}$ & $15.40 \pm 3.89$ & $2134.28 \pm 249.47^{a}$ \\
\hline \multirow{4}{*}{ 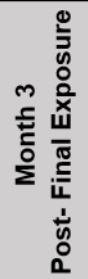 } & CONTROL & $6.60 \pm 3.82$ & $35.37 \pm 5.31$ & $13.96 \pm 3.19$ & $0.09 \pm 0.04$ & $7.17 \pm 1.70$ & $1387.01 \pm 187.80$ \\
\hline & DPM & $10.47 \pm 7.63$ & $12.89 \pm 4.72$ & $13.10 \pm 2.17$ & $0.38 \pm 0.25$ & $7.05 \pm 1.97$ & $1103.68 \pm 269.83$ \\
\hline & SIL & $30.53 \pm 4.87^{c}$ & $32.10 \pm 4.65$ & $177.86 \pm 29.05^{\mathrm{a}}$ & $11.67 \pm 1.68^{a}$ & $41.33 \pm 26.08$ & $3332.88 \pm 278.52^{a}$ \\
\hline & DS & $7.46 \pm 4.42$ & $6.69 \pm 4.64^{c}$ & $104.35 \pm 26.93^{a}$ & $4.39 \pm 1.46^{\mathrm{a}}$ & $16.86 \pm 3.03$ & $2357.64 \pm 306.15^{a}$ \\
\hline
\end{tabular}

All data are shown as mean \pm standard error: ${ }^{a}$ different from control and DPM; ${ }^{b}$ different from control; ${ }^{c}$ different from all other groups; and ${ }^{f}$ different from control and SIL; $p \leq 0.05$. ND: not detected. 
Table 3: Lymphocyte Phenotype of the BALC

\begin{tabular}{|c|c|c|c|c|c|}
\hline \multicolumn{6}{|c|}{ BAL Lymphocytes $\left(\times 10^{-4}\right)$} \\
\hline \multirow{5}{*}{ 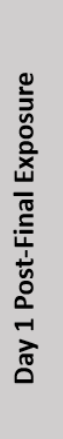 } & $\begin{array}{c}\text { Exposure } \\
\text { Group }\end{array}$ & T Cells & CD4 ${ }^{+} \mathrm{T}$ Cells & CD8 ${ }^{+} \mathrm{T}$ Cells & B Cells \\
\hline & CONTROL & $8.90 \pm 1.42$ & $6.96 \pm 1.16$ & $0.293 \pm 0.0564$ & $5.35 \pm 1.01$ \\
\hline & DPM & $9.57 \pm 1.63$ & $7.16 \pm 1.06$ & $0.441 \pm 0.125$ & $6.28 \pm 1.08$ \\
\hline & SIL & $16.10 \pm 2.56$ & $13.4 \pm 2.11^{\mathrm{a}}$ & $0.838 \pm 0.205$ & $9.78 \pm 0.714$ \\
\hline & DS & $23.70 \pm 3.61^{a}$ & $19.3 \pm 2.93^{\mathrm{a}}$ & $1.44 \pm 0.327^{a}$ & $7.92 \pm 13,500$ \\
\hline \multirow{4}{*}{ 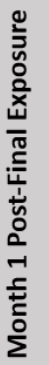 } & CONTROL & $9.72 \pm 1.51$ & $6.74 \pm 1.16$ & $0.928 \pm 0.343$ & $7.00 \pm 0.988$ \\
\hline & DPM & $6.46 \pm 0.929$ & $3.93 \pm 0.483^{b}$ & $0.342 \pm 0.0901^{b}$ & $8.12 \pm 2.55$ \\
\hline & SIL & $28.4 \pm 4.53^{a}$ & $23.6 \pm 3.44^{a}$ & $2.26 \pm 0.585^{d}$ & $11.1 \pm 1.89$ \\
\hline & DS & $60.7 \pm 14.2^{c}$ & $38.9 \pm 6.44^{c}$ & $3.88 \pm 0.519^{c}$ & $11.4 \pm 1.53$ \\
\hline \multirow{4}{*}{ 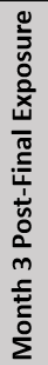 } & CONTROL & $20.1 \pm 2.33$ & $14.8 \pm 1.90$ & $1.31 \pm 0.216$ & $15.0 \pm 1.58$ \\
\hline & DPM & $22.5 \pm 3.56$ & $16.8 \pm 2.79$ & $1.38 \pm 0.479$ & $16.7 \pm 2.97$ \\
\hline & SIL & $51.5 \pm 3.98^{\mathrm{a}}$ & $41.60 \pm 3.33^{a}$ & $4.86 \pm 0.98^{a}$ & $16.0 \pm 2.49$ \\
\hline & DS & $40.9 \pm 6.15^{a}$ & $33.0 \pm 5.49^{\mathrm{a}}$ & $4.05 \pm 0.701^{\mathrm{a}}$ & $15.1 \pm 1.50$ \\
\hline
\end{tabular}

All data are shown as mean \pm standard error: ${ }^{a}$ different from control and DPM; ${ }^{b}$ different from

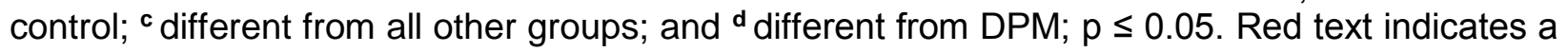
value significantly less than control. 
Table 4: Lymphocyte Phenotype in the MLN

\begin{tabular}{|c|c|c|c|c|c|}
\hline \multicolumn{6}{|c|}{ MLN Lymphocytes $\left(\times 10^{-6}\right)$} \\
\hline \multirow{5}{*}{ 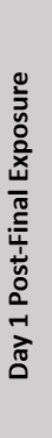 } & $\begin{array}{c}\text { Exposure } \\
\text { Group }\end{array}$ & T Cells & $\mathrm{CD}^{+} \mathrm{T}$ Cells & CD8 $^{+} \mathrm{T}$ Cells & B Cells \\
\hline & CONTROL & $20.2 \pm 1.41$ & $14.9 \pm 1.12$ & $3.32 \pm 1.20$ & $15.3 \pm 1.18$ \\
\hline & DPM & $23.5 \pm 1.88$ & $17.2 \pm 1.80$ & $4.13 \pm 0.893$ & $16.5 \pm 1.72$ \\
\hline & SIL & $25.9 \pm 1.74$ & $18.8 \pm 1.54$ & $5.25 \pm 0.804$ & $19.3 \pm 1.70$ \\
\hline & DS & $34.0 \pm 3.64^{c}$ & $24.2 \pm 2.69^{b}$ & $7.34 \pm 1.49$ & $24.1 \pm 2.58^{a}$ \\
\hline \multirow{4}{*}{ 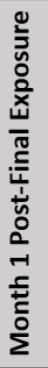 } & CONTROL & $11.4 \pm 0.964$ & $74.0 \pm 0.667$ & $3.36 \pm 0.361$ & $8.57 \pm 0.679$ \\
\hline & DPM & $17.7 \pm 1.99^{b}$ & $11.6 \pm 1.52^{b}$ & $4.54 \pm 0.371^{b}$ & $12.5 \pm 1.99^{b}$ \\
\hline & SIL & $46.7 \pm 2.63^{a}$ & $30.9 \pm 1.75^{a}$ & $13.8 \pm 0.97^{a}$ & $30.6 \pm 1.87^{a}$ \\
\hline & DS & $49.5 \pm 3.77^{a}$ & $30.5 \pm 2.36^{a}$ & $16.9 \pm 1.67^{a}$ & $35.7 \pm 4.22^{\mathrm{a}}$ \\
\hline \multirow{4}{*}{ 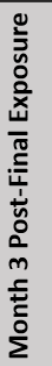 } & CONTROL & $9.12 \pm 0.774$ & $5.92 \pm 0.484$ & $2.89 \pm 0.292$ & $9.87 \pm 1.21$ \\
\hline & DPM & $10.9 \pm 1.81$ & $7.18 \pm 1.21$ & $3.38 \pm 0.555$ & $10.2 \pm 1.33$ \\
\hline & SIL & $48.8 \pm 5.03^{a}$ & $29.4 \pm 3.44^{\mathrm{a}}$ & $18.1 \pm 1.69^{a}$ & $38.6 \pm 5.25^{a}$ \\
\hline & DS & $67.7 \pm 7.58^{\mathrm{a}}$ & $41.7 \pm 4.97^{a}$ & $23.9 \pm 2.78^{a}$ & $60.8 \pm 7.99^{c}$ \\
\hline
\end{tabular}

All data are shown as mean \pm standard error: ${ }^{\mathbf{a}}$ different from control and DPM; ${ }^{\mathbf{b}}$ different from control; and ${ }^{\mathrm{c}}$ different from all other groups; $p \leq 0.05$. 
Table 5: Lung Histopathology Severity and Incidence Scores

\begin{tabular}{|c|c|c|c|c|c|c|}
\hline \multicolumn{7}{|c|}{ Histopathology Means and Incidences } \\
\hline \multirow{5}{*}{ 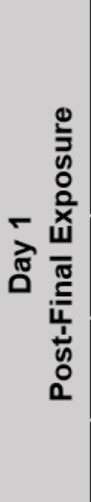 } & $\begin{array}{c}\text { Exposure } \\
\text { Group }\end{array}$ & AM & AEH & MI & $\mathbf{F}$ & BG \\
\hline & Control & $0(0 / 5)$ & $0(0 / 5)$ & $0(0 / 5)$ & $0(0 / 5)$ & $0(0 / 5)$ \\
\hline & DPM & $0(0 / 5)$ & $0(0 / 5)$ & $0(0 / 5)$ & $0(0 / 5)$ & $1(5 / 5) \mathrm{D}^{\mathrm{f}^{*}}$ \\
\hline & SIL & $0(0 / 5)$ & $0(0 / 5)$ & $1.4(5 / 5) \mathrm{M}^{\mathrm{c} *}$ & $0(0 / 5)$ & $0(0 / 5)$ \\
\hline & DS & $0(0 / 5)$ & $0(0 / 5)$ & $0.2(1 / 5) \mathrm{M}$ & $0(0 / 5)$ & $1.4(5 / 5) D^{f^{*}}$ \\
\hline \multirow{4}{*}{ 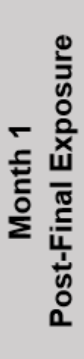 } & Control & $0(0 / 5)$ & $0(0 / 5)$ & $0.4(2 / 5) \mathrm{D}$ & $0(0 / 5)$ & $0(0 / 5)$ \\
\hline & DPM & $0(0 / 5)$ & $0(0 / 5)$ & $0(0 / 5)$ & $0(0 / 5)$ & $1.2(5 / 5) D^{f^{*}}$ \\
\hline & SIL & $1(4 / 5) M^{a}$ & $0(0 / 5)$ & $1.2(4 / 5) \mathrm{M}^{\mathrm{d}}$ & $0(0 / 5)$ & $0(0 / 5)$ \\
\hline & DS & $0.2(1 / 5) \mathrm{M}$ & $0(0 / 5)$ & $1.6(5 / 5) \mathrm{M}^{\text {a† }}$ & $0.4(2 / 5) \mathrm{M}$ & $1.8(5 / 5) M^{f^{*}}$ \\
\hline \multirow{4}{*}{ 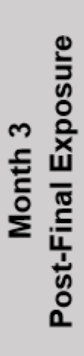 } & Control & $0(0 / 5)$ & $0(0 / 5)$ & $0(0 / 5)$ & $0(0 / 5)$ & $0(0 / 5)$ \\
\hline & DPM & $0(0 / 5)$ & $0(0 / 5)$ & $0.2(1 / 5) \mathrm{M}$ & $0(0 / 5)$ & $0(0 / 5)$ \\
\hline & SIL & $0.6(2 / 5) \mathrm{M}$ & $0(0 / 5)$ & $1.6(5 / 5) \mathrm{M}^{\mathrm{att}}$ & $0(0 / 5)$ & $0(0 / 5)$ \\
\hline & DS & $1.6(4 / 5) \mathrm{M}^{\mathrm{a}}$ & $0.8(4 / 5) \mathrm{M}^{\mathrm{c}}$ & $1.8(5 / 5) \mathrm{M}^{\mathrm{att}}$ & $0.8(4 / 5) \mathrm{M}^{\mathrm{c}}$ & $1(5 / 5) M^{c}$ \\
\hline
\end{tabular}

Lung tissue was analyzed and scored for accumulation of alveolar macrophages (AM), alveolar epithelial hyperplasia (AEH), brown granules in alveolar macrophages (BG; presence of DPM particles), mixed cellular infiltration (MI), and fibrosis in alveolar septae $(F)$ ( $n=5$ per group per time point). Severity was scored as $0-5: 0=$ normal, $1=\mathrm{minimal} / \mathrm{slight}, 2=\mathrm{mild}, 3=$ moderate, $4=$ marked, and 5 = severe. Incidence was noted as focal (F), multi-focal (M), and diffuse (D). Data are presented as means with incidence (number of animals with a positive score per total animals) in parentheses. 'Different from control and DPM groups; ' different from all other groups; different from DPM only; and fdifferent from control and SIL groups; $p$ value $<0.05$. Additional symbols indicate ${ }^{*} p<0.01 ;{ }^{* *} p<0.01$ compared with control and DPM; ${ }^{\dagger} p<0.01$ compared with DPM; and ${ }^{\mathrm{t}} \mathrm{p}<0.01$ compared with control. 
Figure 1:

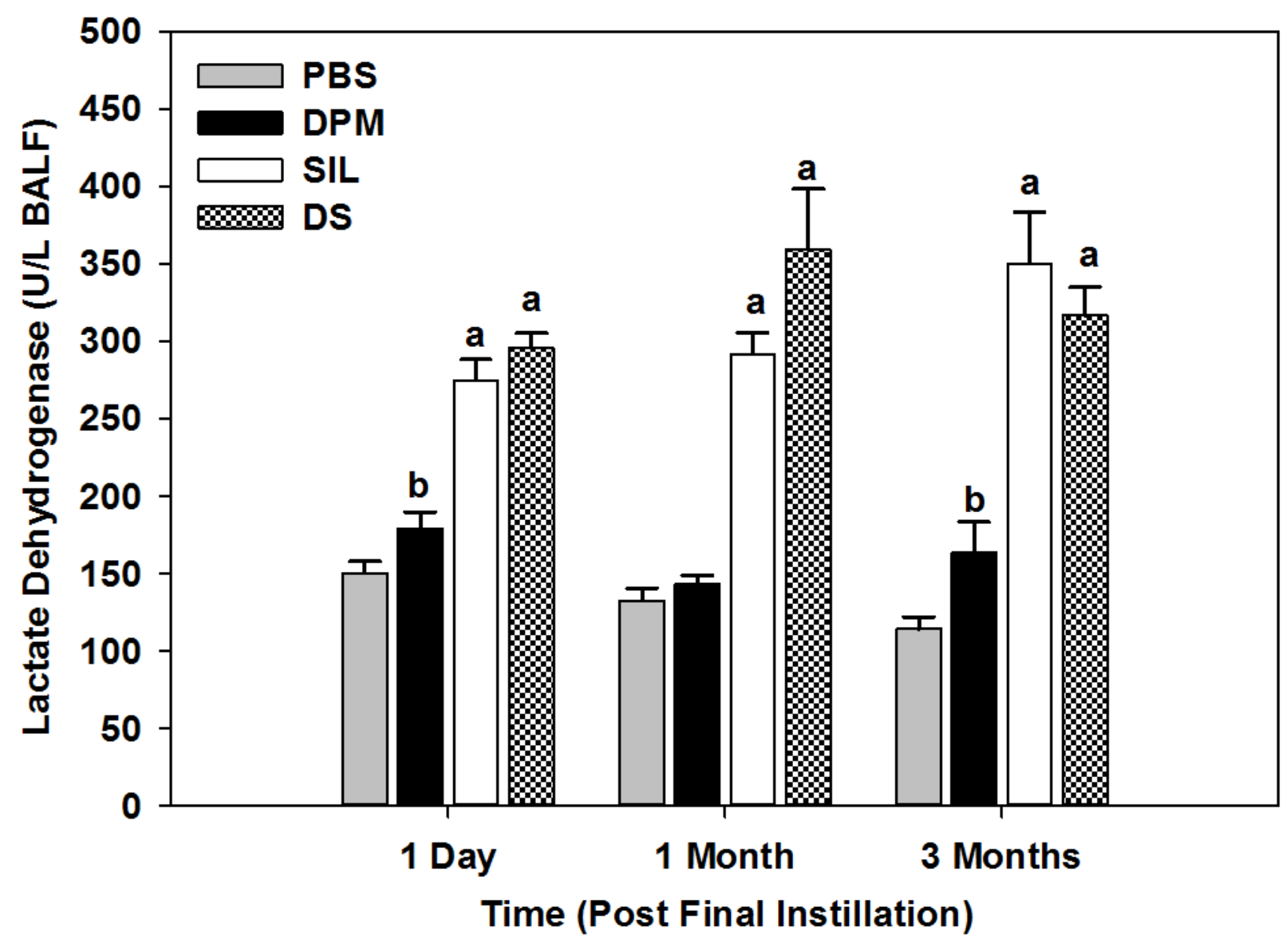

Figure 1: Lactate dehydrogenase activity (LDH) in BALF after exposure to PBS, DPM, SIL, or a combination of DPM and SIL (DS). All data are shown as mean \pm standard error: ${ }^{a}$ different from control and DPM; ${ }^{b}$ different from control; $p \leq 0.05$. 
Figure 2:

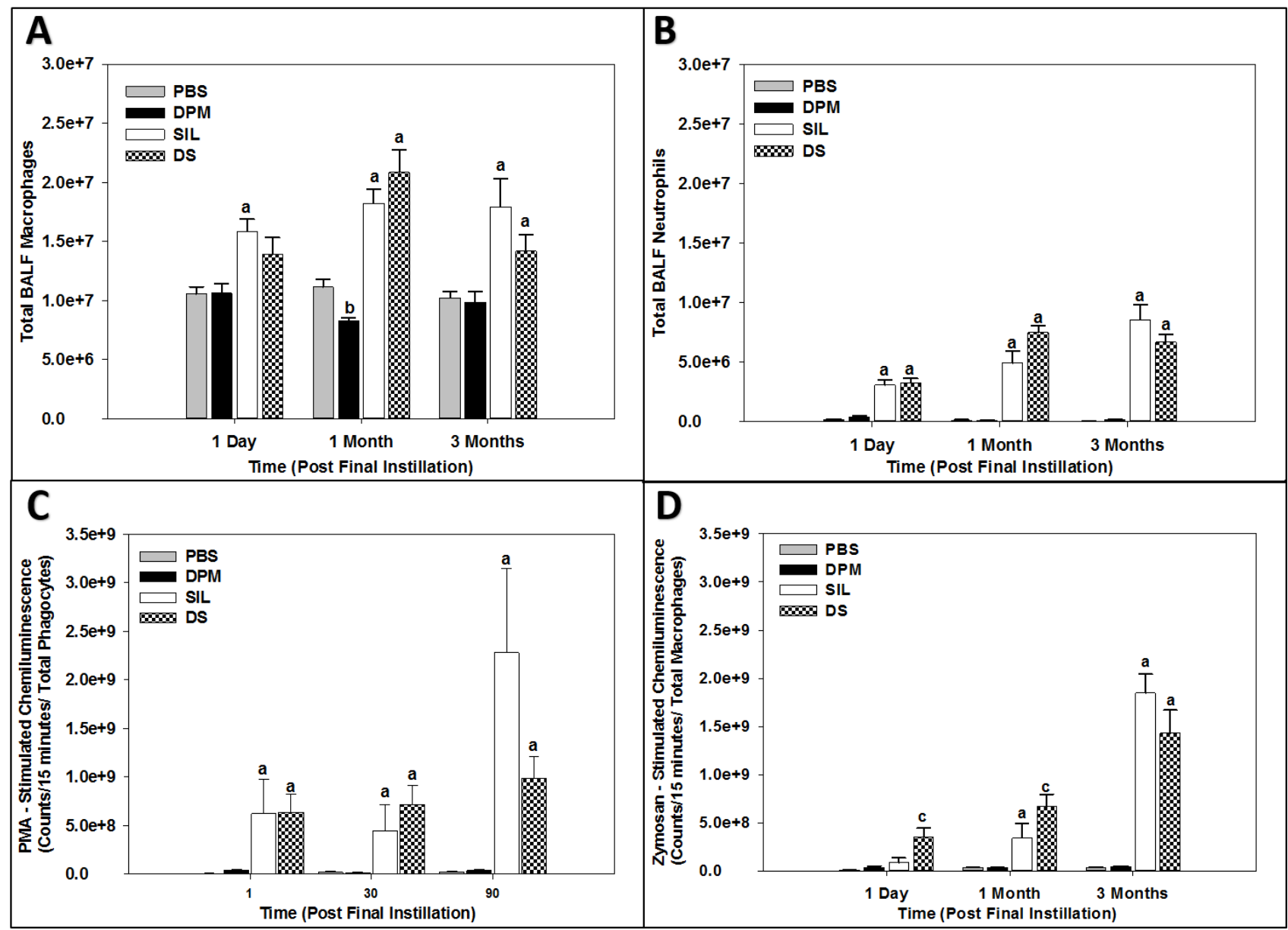

Figure 2: Total macrophages (A) and neutrophils (B) in the BALF following exposure to PBS, DPM, SIL, or DS. Production of oxidants by total phagocytes stimulated with PMA (C) or by macrophages only stimulated by zymosan (D) measured by chemiluminescence following exposure to PBS, DPM, SIL, or DS. All data are shown as mean \pm standard error: ${ }^{a}$ different from control and DPM; ${ }^{\text {b different }}$ from control; and 'different from all groups; $p \leq 0.05$. 


\section{Figure 3:}

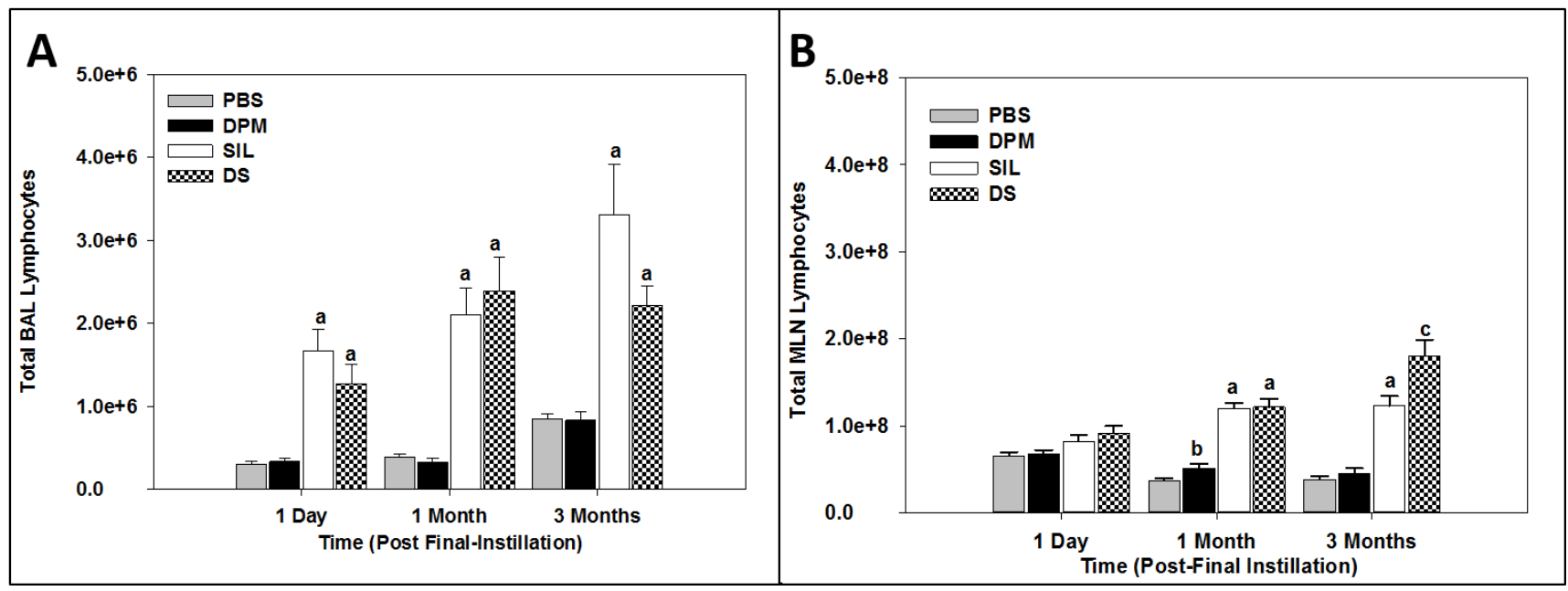

Figure 3: Total BAL Lymphocytes (A) and MLN Lymphocytes (B) following exposure to PBS, DPM,

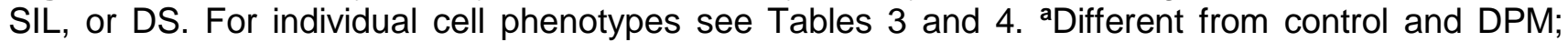
${ }^{b}$ different from control only; and 'different from all groups. Statistical significance measured as $p \leq$ 0.05 . 
Figure 4:

Day 1 Month $1 \quad$ Month 3

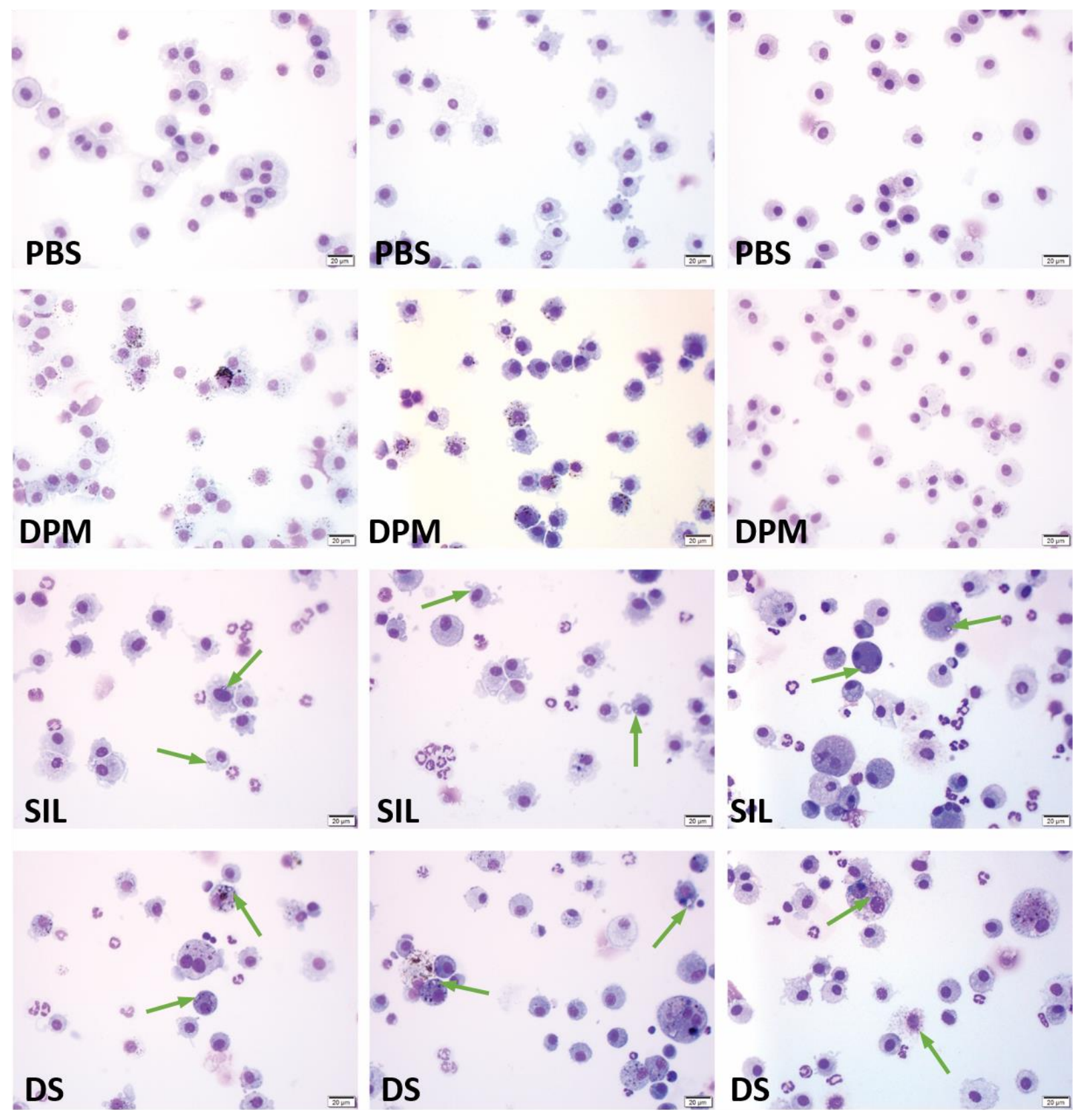

Light field microscopy images of cytospins used to qualitatively assess particulate clearance by BAL AMs at 1 day, 1 month, and 3 months following instillation. Images were taken at 40x magnification and scale bar represents $20 \mu \mathrm{m}$ in length. Green arrows indicate the presence of silica particulates within AMs. 
Figure 5:
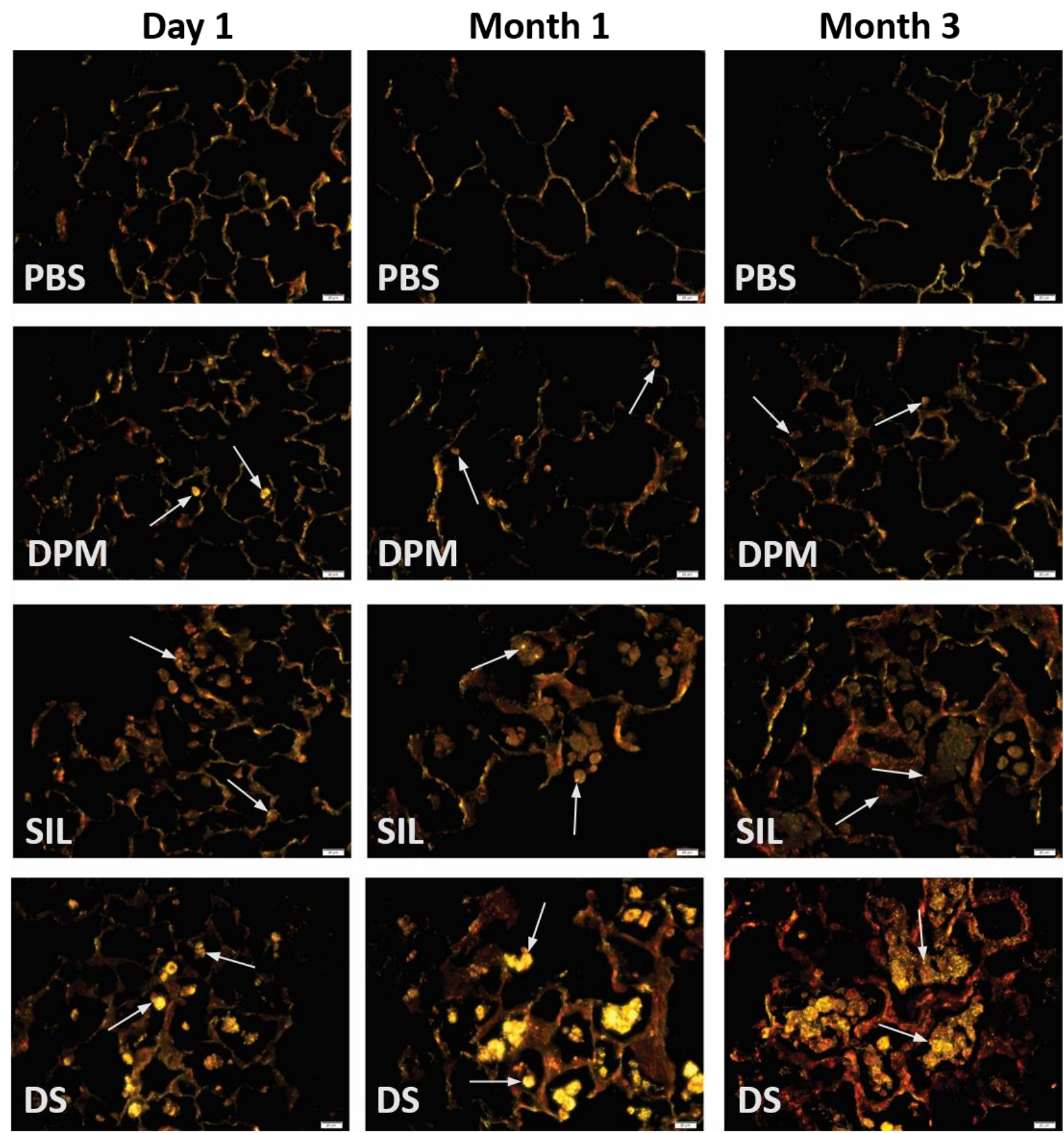

Figure 5: Dark field microscopy images of Sirius red stained tissue used to qualitatively assess tissue injury and particulate loaded cells at 1 day, 1 month, and 3 months following instillation. Images were taken at 20x magnification and scale bar represents $20 \mu \mathrm{m}$ in length. White arrows indicate the presence of particulate within macrophages. 
Figure 6:

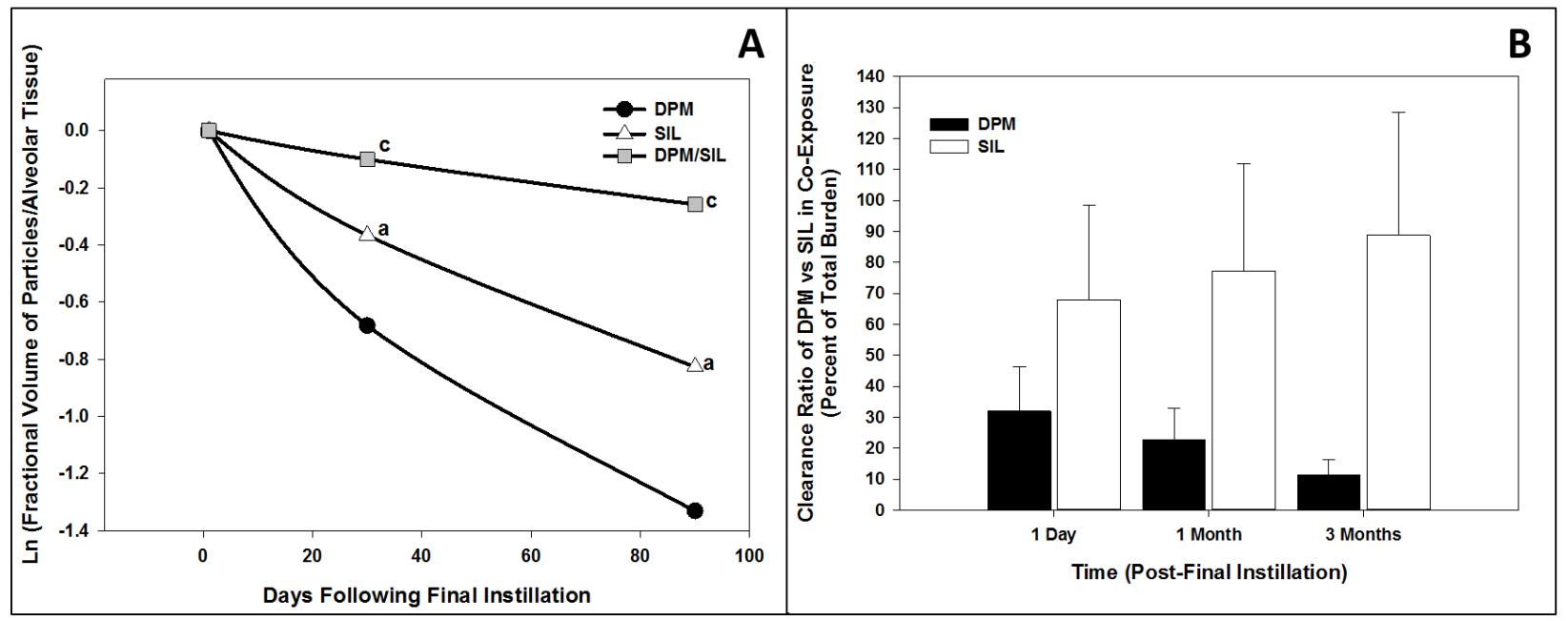

Figure 6: Particle clearance for each dosing group as quantitatively evaluated by morphometry; data was normalized to 1.0 for day one following the last instillation in order to compare groups that received variable particle masses of initial dose; data shown is Ln of normalized data. For each group an $n=5$ tissue slides per time-point were morphometrically assessed (A). Relative ratio of each particulate remaining in the co-exposure group at each time-point as assessed by morphometry (B). 


\section{Chapter 5}

\section{Overall Discussion}




\section{Discussion}

Industrial operations such as above and below-ground mining may result in occupational respiratory exposure to complex mixtures of particulates including crystalline silica and diesel exhaust. Toxicity following pulmonary exposure to either crystalline silica or diesel exhaust have been studied in detail as individual exposures, as extensively reviewed by several investigators $[4,5,21,128,306,465,511,550-552,559-561]$. Silica has been shown to cause oxidative stress, prolonged inflammation, development of restrictive fibrotic lung disease, hyperresponsiveness of some $T$ cell subsets, upregulation of the inflammasome, incidence of autoimmune developments, increased susceptibility to infections, and cancers. Diesel exposure may result in oxidative stress, inflammation, suppression of macrophage function, reduced T cell activity, increased susceptibility to infection, allergic sensitization, development of obstructive lung diseases, and cancer. The hazards associated with these exposures are wellrecognized and regulatory organizations have recommended exposure limits (RELs) [30, 184], permissible exposure limits (PELs) [7, 31, 182], or occupational exposure limits (OELs) [179181,183 ] for crystalline silica and in some instances for underground levels of diesel (regulated as total carbon). However, there are very few studies that investigate the health effects of these two materials in a co-exposure scenario that may occur in occupational setting, such as surface or below-ground mining operations, including hydraulic fracturing, a fast-expanding form of oil and gas extraction that utilizes diesel-powered engines as the motive force in moving proppant and sand containing crystalline silica.

The current series of investigations was designed to address these main knowledge gaps in the literature. The overall goal of these studies was to characterize pulmonary toxicity following a co-exposure to diesel particulate matter (DPM) and crystalline silica (SIL) at doses derived from current occupational measures, and determine if these effects were variable compared with exposure to the particles individually. The project had three major aims: (1a) Characterize the pulmonary response to an acute co-exposure to DPM and SIL at varying doses; (1b) characterize the pulmonary response to a repeated co-exposure to DPM and SIL in order to determine if subacute exposure vs. acute exposure altered the effects observed for 1a; (2) establish if susceptibility to an acute respiratory infection was altered following a coexposure of DPM and SIL; and (3) determine whether clearance of particulate in a co-exposure to DPM and SIL was altered compared with the clearance rate of either particle individually to assess the effect of particle load in toxicity 
In Study 1, two doses of DPM were employed, which were derived from field measures of elemental carbon (EC) collected either above or below-ground during mining operations [547]. Particulates were administered to rats by intratracheal instillation in one bolus dose to model an acute exposure. The study showed that these doses of DPM, 7 or $50 \mu \mathrm{g}$, produced low to no effect on pulmonary toxicity individually. The high dose of SIL $(233 \mu \mathrm{g})$, which was greater than that of the DPM by mass, but not the lower dose $(50 \mu \mathrm{g})$, did result in acute injury, inflammation, alterations in immune response, and the induction of pathological changes in the lung associated with fibrosis. When the higher of the two DPM doses was combined with the high dose of SIL, inflammation, and oxidant production by phagocytes were enhanced at early time-points. By 3 months post-exposure, effects of the co-exposure were similar to that of SIL alone, which persisted or progressed depending on the parameter of toxicity. This led to the goal of determining whether the increase in toxic effects elicited by DPM at earlier time-points were not enhanced beyond that of SIL at 3 months following exposure due to clearance of DPM, or a masking of any additional effect of DPM due to the progression of toxicity caused by SIL. Additionally, there was a need to address whether the effects observed were due to increased particle load administered to the co-exposure group, or rather, due to specific properties of DPM in the co-exposure.

This concept was addressed in Study 2, where equal doses of SIL, and DPM or CB were administered to determine if the effects observed in Study 1 were particle-specific to DPM or if total particle load was a factor in toxicity. CB was employed as a particle load control for comparison to DPM as it is comprised of the primary core component of DPM, but does not have the metal and PAH components, and it is relatively low in pulmonary toxicity at nonoverload doses [267, 277, 281]. It was determined from this investigation that DPM given at a dose equivalent to that of silica, also enhanced inflammation, injury, oxidant production, and induction of pathological changes observed for silica alone. Further, CB did not contribute to enhanced inflammation, injury, and oxidant production beyond that of silica alone suggesting that for DPM and silica combined, these responses were due to specific characteristics of DPM and not due specifically to an increase in overall particle load. However, pathological changes were closely correlated to SIL exposure, but were enhanced by particle load as these changes were slightly increased beyond that of SIL for both DPM and CB co-exposed groups. Further, it was established that overall clearance of particles was reduced in the co-exposures compared with individual particles, which may suggest worsened outcomes for more chronic co-exposure scenarios. 
Study 3 investigated toxicological changes that occur during a sub-chronic, repeated coexposure to lower concentrations of particles representing a longer period of working conditions from mining sites. Rats were exposed intratracheally to DPM ( $25 \mu \mathrm{g})$, SIL (116 $\mu \mathrm{g})$, or DPM (25 $\mu \mathrm{g})$ and SIL $(116 \mu \mathrm{g})$ combined once per week for four consecutive weeks. Effects observed in the co-exposure group in this study were primarily due to silica, as this group did not differ from SIL alone as had been observed in the acute dose study; however, relatively low doses of DPM were sufficient to enhance the production of oxidants by phagocytes at 1 month following final exposure, and increase the incidence of fibrotic pathological changes in the lung tissue beginning at 1 month following exposure that increased further at 3 months following final exposure. Additionally, it was observed that DPM both alone, and in co-exposure with SIL was capable of eliciting independent action on the expression of some proteins in the lung associated with chemotaxis of innate immune cells and the differentiation of lymphocytes.

Based on the findings related to immune response in the characterization studies, further studies examining the susceptibility to infection were designed to determine if alterations of inflammation, oxidant production, and protein expression might alter pulmonary response to a pathogen using the acute exposure models in studies 1 and 2. Although bacterial clearance was reduced for the highest dose of DPM at day 3 following infection when compared to all groups that contained SIL, all exposed groups were able to clear infection in the adaptive phase of the immune response following exposure to bacteria. The high dose DPM group showed a degree of increased T cell response to infection at days 5 and 7 (Study 2, Table 7), although not to the level of significance, which may have been compensatory to the initial suppression in innate immune response and increased bacterial load at day 3. Therefore, the differential effects on immune parameters of DPM in the co-exposure groups observed in studies 1 and 2 in the absence of infection were not significant enough to disrupt immune function in the response to a pathogen.

Taken together, these results suggest that acute exposure to DPM and SIL in combination showed that relatively low toxicity doses of DPM could exacerbate the effects of lung injury and inflammation and alter immune responses when compared to that of SIL exposure alone, while total particle load in the presence of SIL contributed to fibrotic response and tissue remodeling. Further, the finding that combined exposure to DPM and SIL results in reduced clearance rate of particles when compared to individual exposures may have implications in development of adverse pulmonary outcomes following chronic exposures, where persistent particle load could further exacerbate lung pathology. It is well-accepted that 
particle clearance and load are critical factors in toxicity, and that particle overload can be a mechanism of toxicity for particles that are considered relatively non-toxic or "nuisance dusts." Overload is described as a particle load that overwhelms the capability of natural clearance mechanisms by way of mucociliary escalation or uptake by macrophages, and is dependent on a number of particle properties including size, shape, and density, occurring when a macrophage reaches a particle burden of $~ 60 \%$ of its volume $[75,562]$.

Although combined exposure to DPM and SIL led to reduced rate of particle clearance, continued particle clearance was observed throughout the time course suggesting that the doses of particles administered did not reach overload. Also, although there were macrophages that were in particle overload conditions at the highest doses of DPM administered in study 2 , the vast majority of macrophages recovered by BAL did not have greater than $60 \%$ volume in particles. It is important to note that the instillation doses used in these studies better reflect doses delivered by inhalation as calculated based on worksite measurements and pulmonary deposition models; whereas, historically, intratracheal instillation studies of silica and diesel individually have used much higher acute bolus doses of the materials to represent longer, more chronic exposures or to determine maximal effect of toxicity [291, 468, 521, 563-567]. Therefore, bolus dose delivery often reflect amounts of particulate that far-exceed exposure levels that are likely to occur routinely in the workplace, and may reflect to some degree the state of particle overload, which may not adequately reflect workplace exposures where particle concentrations are kept within the limitations outlined by governing agencies [31, 180-183]. The reduced rate of particle clearance observed in the co-exposures in the present studies are not a product of particle overload in the lung, which allows the investigators to be able to determine effects that are correlated to particle specific toxicity or general particle load effects under nonoverload conditions.

Reduced clearance of a highly toxic material, such as silica, following exposure is of significant concern. As silica is known to be directly cytotoxic and reactive, is it not surprising that parameters of toxicity associated with silica, such as inflammation and cytotoxicity, are dose and exposure duration dependent [568-570]. It has been shown that once a given threshold of silica exposure has been reached and exposure has ceased, progression of pathology may still occur $[74,80,81,570]$. This was also observed in the SIL group in the current studies; whereas, the dose-dependent effects for DPM alone resolved over time [547]. In study 1 and study 2 , the total particle load increased in the co-exposure groups over the individual exposures on a mass basis, and although several parameters measured showed a 
particle-specific effect due to DPM, other parameters were influenced more by particle load and the presence of SIL. Tissue remodeling and fibrosis, although dependent upon the presence of SIL, were increased due to overall particle load, as the DPM or CB co-exposures did not differ from each other and resulted in increased tissue injury over that of SIL alone. This suggests that particulate combined with a toxic dose of SIL may be sufficient to elicit these effects, regardless of the specific particle.

Overall, these studies show particle specific effects of DPM as well as particle load effects in the co-exposure; however the majority of the underlying toxicity during this investigation was initiated by SIL. As mentioned, inflammation, cytotoxic injury, migration of immune cells into the lung, proliferation of lymphocytes in MLN, production of oxidants by phagocytes, and expression of inflammatory and tissue remodeling proteins in the lung were increased in response to silica at the high dose $(233 \mu \mathrm{g})$ throughout the time course. These results are in agreement with numerous studies examining the pulmonary toxicity of silica [80, $81,571]$. In sharp contrast to the effects of silica, the doses of DPM used in our studies ( 7, 50, or $233 \mu \mathrm{g}$ ) did not elicit persistent inflammatory effects beyond 1 day or 1 week for most parameters when administered as an individual exposure, although DPM did appear to alter effects related to immune function differentially from that of SIL. The transient inflammatory responses are in contrast to other investigations of DPM toxicity [268-272], and this may be due to several factors including dose, whereby the doses used in the present study are lower than those employed in other installation studies, as well as duration of exposure (an acute or subacute dose versus subchronic or chronic studies), and/or composition of diesel depending on source, engine type, whole exhaust, or particulate fraction [278, 280, 308, 572].

Immune effects related to DPM are most often associated with suppression of certain parameters of innate immunity related to macrophage effector functions [248, 316, 318, 324, $325,387,573,574]$. In the current studies, we did observe decreased influx of macrophages following subacute exposure and the expression of inflammatory cytokines associated with macrophage effector functions for both acute and subacute exposure, specifically signaling chemotaxis of additional immune cells and auto-activation. Furthermore, DPM exposure resulted in the decreased influx of some sets of $T$ cells into the lung corresponding with a decrease in IP-10 at 1 month following final exposure in our subacute model. Interestingly, some suppression of macrophage related proteins was also observed in the DPM and SIL combined exposure as compared with SIL exposure alone, suggesting that DPM has the capability to attenuate the effects observed for SIL depending on the time point and parameter 
examined, which may play a role in susceptibility to infection during a chronic model of exposure and infection. Though an alteration in bacterial clearance following infection was not observed for the lower dose of DPM $(50 \mu \mathrm{g})$, the higher dose exposure group $(233 \mu \mathrm{g})$ had significantly decreased clearance at day 3 following infection, which was not observed for the same particle load of CB (Study 2). Moreover, clearance of bacteria in the DPM/SIL group was not different from that of SIL.

Overall, the suppressive effects of DPM in the present studies were transient and, although there were clear trends in the suppression of macrophage response, not all parameters were statistically significant from control, as has been observed in other studies of diesel reviewed in Chapter 1 [387, 573, 574]. As mentioned above, the differences in the degree of immune suppression observed in other investigations versus the current studies described here may be attributable to the form of diesel used in this study. Investigators have demonstrated that the immunoregulatory effects of DPM are more closely related to the organic fraction of the material adsorbed to the carbon core of DPM [268, 296, 298, 324, 325, 330, 335, 337, 339], and not to the carbon core itself, which is similar to the CB control used in study 2. Relative to other studies, the diesel exposure in this study used only the particulate form diesel collected from a forklift (NIST SRM 2975) representing a "tranisitional" form of diesel exhaust. This particle is relatively low in organic material relative to freshly generated diesel exhaust. The studies did show, however, a degree of suppression in macrophage- and immune-related responses over that of the carbon core control group (CB), suggesting that the levels of components adsorbed to the core of the DPM in this study may contribute to the particle specific effects related to immune parameters in the co-exposure group.

Taken together, the data indicate that toxicity in the co-exposure group was attributable to SIL, and although there was a degree of independent action due to DPM in the co-exposure group in regard to immune function, DPM did have particle-specific effects in the co-exposure group related to exacerbation of inflammation and oxidant production; whereas, histopathological changes were primarily attributable to particle load and the presence of SIL. These studies are the first to characterize effects that are specific to the interaction of DPM with SIL in a co-exposure and are important for the continued understanding of workplace exposures, which are relevant to a wide number of occupations. 


\section{Model for Overall Progression of Lung Toxicity}

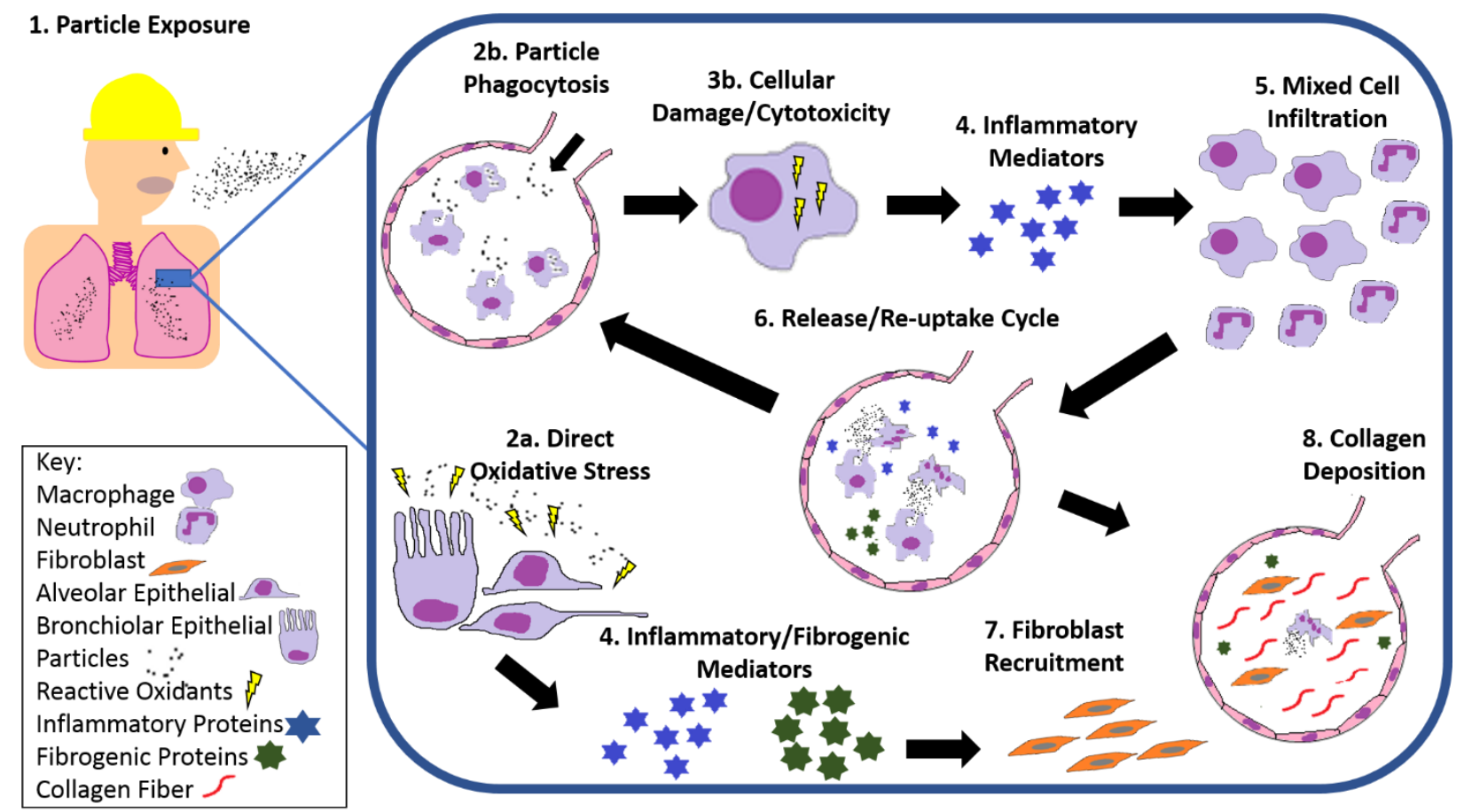

1) A worker is exposed to silica particles. 2) Silica particles may cause direct damage to bronchiolar or alveolar epithelial cells in the form of reactive oxidants leading to oxidative stress (2a), or may be phagocytosed by cells such as alveolar macrophages when deposited deeply within the lung (2b). 3) Particles that are phagocytosed will result in cellular damage in the form of reactive oxidants that may cause cellular damage (oxidative stress) or cell death. 4) Cells, either bronchial or alveolar epithelial, or cells that have phagocytosed particles will express inflammatory mediators and/or fibrogenic mediators. 5) Inflammatory mediators signal the infiltration of additional immune cells such as monocytes, macrophages, and neutrophils. 6) Cell damage/death leads to the re-release of silica particles into the alveolar spaces, which must be phagocytosed by newly recruited cells. This process is reinitiated again at 2b. 7) Fibrogenic mediators recruit fibroblasts to the site of prolonged inflammation in order to secrete extracellular matrix proteins such as collagen in an effort to sequester silica particles to a particular area. 8) Collagen deposition results in the development of fibrosis. This study also showed that exposure to DPM in addition to silica resulted in more prolonged and increased parameters of inflammation, cellular injury, and oxidative stress. Furthermore, the increase in particle load resulted in an early progression of mild alveolar epithelial hyperplasia and mild fibrosis. 


\section{Bibliography}

1. Rosen, G., The history of miners' diseases, a medical and social interpretation, by George Rosen ... With an introduction by Henry E. Sigerist. 1943, New York: Schuman's. xii, 490 p.

2. Collis, E.L., Industrial pneumonoconioses, with special reference to dust-phthisis. Public Health, 1915. 28(4): p. 252-264.

3. Laney, A.S., E.L. Petsonk, and M.D. Attfield, Pneumoconiosis among underground bituminous coal miners in the United States: is silicosis becoming more frequent? Occup Environ Med, 2010. 67(10): p. 652-6.

4. Castranova, V. and V. Vallyathan, Silicosis and coal workers' pneumoconiosis. Environmental Health Perspectives, 2000. 108(Suppl 4): p. 675-684.

5. Steiner, S., et al., Diesel exhaust: current knowledge of adverse effects and underlying cellular mechanisms. Arch Toxicol, 2016. 90(7): p. 1541-53.

6. CATF, Occupational Exposure to Diesel Exhaust, C.A.T. Force, Editor. 2008.

7. OSHA, Worker's Exposure to Respirable Crystalline Silica: Final Rule Overview, in OSHA Fact Sheet. 2016, United States Department of Labor: Online.

8. Yassin, A., F. Yebesi, and R. Tingle, Occupational Exposure to Crystalline Silica Dust in the United States, 1988-2003. Environmental Health Perspectives, 2005. 113(3): p. 255-260.

9. Cohen, R.A.C., A. Patel, and F.H.Y. Green, Lung Disease Caused by Exposure to Coal Mine and Silica Dust. Semin Respir Crit Care Med, 2008. 29(06): p. 651-661.

10. Möhner, M., N. Kersten, and J. Gellissen, Chronic obstructive pulmonary disease and longitudinal changes in pulmonary function due to occupational exposure to respirable quartz. Occupational and Environmental Medicine, 2012. 70(1): p. 9.

11. Hnizdo, E. and V. Vallyathan, Chronic obstructive pulmonary disease due to occupational exposure to silica dust: a review of epidemiological and pathological evidence. Occupational and Environmental Medicine, 2003. 60(4): p. 237.

12. Steenland, K. and E. Ward, Silica: a lung carcinogen. CA Cancer J Clin, 2014. 64(1): p. 63-9.

13. Ojajarvi, I.A., et al., Occupational exposures and pancreatic cancer: a meta-analysis. Occup Environ Med, 2000. 57(5): p. 316-24.

14. Pan, G., et al., Nested case-control study of esophageal cancer in relation to occupational exposure to silica and other dusts. Am J Ind Med, 1999. 35(3): p. 272-80.

15. Sjodahl, K., et al., Airborne exposures and risk of gastric cancer: a prospective cohort study. Int J Cancer, 2007. 120(9): p. 2013-8.

16. Tsuda, T., et al., A case-control study of the relationships among silica exposure, gastric cancer, and esophageal cancer. Am J Ind Med, 2001. 39(1): p. 52-7.

17. Chen, W., et al., Long-Term Exposure to Silica Dust and Risk of Total and Cause-Specific Mortality in Chinese Workers: A Cohort Study. PLOS Medicine, 2012. 9(4): p. e1001206.

18. Liu, Y., et al., Long-term exposure to crystalline silica and risk of heart disease mortality. Epidemiology, 2014. 25(5): p. 689-96.

19. Dong, D., et al., Lung cancer among workers exposed to silica dust in Chinese refractory plants. Scand J Work Environ Health, 1995. 21 Suppl 2: p. 69-72.

20. Weiner, J., L. Barlow, and B. Sjogren, Ischemic heart disease mortality among miners and other potentially silica-exposed workers. Am J Ind Med, 2007. 50(6): p. 403-8.

21. Leung, C.C., I.T. Yu, and W. Chen, Silicosis. Lancet, 2012. 379(9830): p. 2008-18. 
22. Middleton, E.L., THE PRESENT POSITION OF SILICOSIS IN INDUSTRY IN BRITAIN. British Medical Journal, 1929. 2(3584): p. 485-489.

23. Agricola, G., De re metallica / Georgius Agricola ; translated from the first Latin edition of 1556, with biographical introduction, annotations and appendices, ed. H. Hoover and L.H. Hoover. 1950, New York: Dover Publications.

24. Cherniak, M., The Hawk's Nest incident : America's worst industrial disaster. 1986, New Haven; London: Yale University Press.

25. ILO, Guidelines for the use of the ILO International Classification of Radiographs of Pneumoconioses (OSH 22). 2011, International Labour Office: Geneva :.

26. Delgado García, D.D., Silicosis: controversy in detection. Medicina y Seguridad del Trabajo, 2014. 60: p. 4-8.

27. Castranova, V., Particulates and the Airways: Basic Biological Mechanisms of Pulmonary Pathogenicity. Applied Occupational and Environmental Hygiene, 1998. 13(8): p. 613-616.

28. Vallyathan, V., et al., Generation of free radicals from freshly fractured silica dust. Potential role in acute silica-induced lung injury. Am Rev Respir Dis, 1988. 138(5): p. 1213-9.

29. IARC, IARC Monographs on the Evaluation of Carcinogenic Risks to Humans. 2012, World Health Organization: International Agency for Research on Cancer: Online.

30. NIOSH, Criteria for a Recommended Standard: Occupational Exposure to Crystalline Silica, D.o.H.E.a. Welfare, Editor. 1974: National Institute for Occupational Safety and Health.

31. OSHA, Occupational Exposure to Respirable Crystalline Silica, in 29 CFR Parts 1910, 1915, and 1926, U.S.F. Government, Editor. 2016, Department of Labor: Federal Register. p. 605.

32. Attfield, M.D. and J. Costello, Quantitative exposure-response for silica dust and lung cancer in Vermont granite workers. American Journal of Industrial Medicine, 2004. 45(2): p. 129-138.

33. Preller, L., et al., Occupational exposure to silica and lung cancer risk in the Netherlands. Occup Environ Med, 2010. 67(10): p. 657-63.

34. Liu, Y., et al., Exposure-response analysis and risk assessment for lung cancer in relationship to silica exposure: a 44-year cohort study of 34,018 workers. Am J Epidemiol, 2013. 178(9): p. 142433.

35. Kachuri, L., et al., Occupational exposure to crystalline silica and the risk of lung cancer in Canadian men. Int J Cancer, 2014. 135(1): p. 138-48.

36. Elci, O.C., et al., Occupational dust exposure and the risk of laryngeal cancer in Turkey. Scand J Work Environ Health, 2002. 28(4): p. 278-84.

37. Elci, O.C. and M. Akpinar-Elci, Occupational exposures and laryngeal cancer among non-smoking and non-drinking men. Int J Occup Environ Health, 2009. 15(4): p. 370-3.

38. Sartor, S.G., et al., [Occupational risks for laryngeal cancer: a case-control study]. Cad Saude Publica, 2007. 23(6): p. 1473-81.

39. Maeda, M., et al., Dysregulation of the immune system caused by silica and asbestos. J Immunotoxicol, 2010. 7(4): p. 268-78.

40. ATS, Adverse effects of crystalline silica exposure. American Thoracic Society Committee of the Scientific Assembly on Environmental and Occupational Health. Am J Respir Crit Care Med, 1997. 155(2): p. 761-8.

41. Koeger, A.C., et al., Silica-associated connective tissue disease. A study of 24 cases. Medicine (Baltimore), 1995. 74(5): p. 221-37.

42. Makol, A., M.J. Reilly, and K.D. Rosenman, Prevalence of connective tissue disease in silicosis (1985-2006)-a report from the state of Michigan surveillance system for silicosis. Am J Ind Med, 2011. 54(4): p. 255-62. 
43. Blanc, P.D., B. Jarvholm, and K. Toren, Prospective risk of rheumatologic disease associated with occupational exposure in a cohort of male construction workers. Am J Med, 2015. 128(10): p. 1094-101.

44. Beaudreuil, S., et al., Occupational exposure in ANCA-positive patients: a case-control study. Kidney Int, 2005. 67(5): p. 1961-6.

45. Shtraichman, O., et al., Outbreak of autoimmune disease in silicosis linked to artificial stone. Occup Med (Lond), 2015. 65(6): p. 444-50.

46. Parks, C.G., et al., Occupational exposure to crystalline silica and risk of systemic lupus erythematosus: a population-based, case-control study in the southeastern United States. Arthritis Rheum, 2002. 46(7): p. 1840-50.

47. Bovenzi, M., et al., Scleroderma and occupational exposure. Scand J Work Environ Health, 1995. 21(4): p. 289-92.

48. Hayashi, H., et al., Dysregulation of autoimmunity caused by silica exposure and alteration of Fas-mediated apoptosis in T lymphocytes derived from silicosis patients. Int J Immunopathol Pharmacol, 2011. 24(1 Suppl): p. 11s-16s.

49. Otsuki, T., et al., Soluble Fas mRNA is dominantly expressed in cases with silicosis. Immunology, 1998. 94(2): p. 258-62.

50. Wu, P., et al., Reduced function of $C D 4+25+$ regulatory $T$ cell fraction in silicosis patients. Int J Immunopathol Pharmacol, 2006. 19(2): p. 357-68.

51. Otsuki, T., et al., Alterations of Fas and Fas-related molecules in patients with silicosis. Exp Biol Med (Maywood), 2006. 231(5): p. 522-33.

52. Rocha-Parise, M., et al., Lymphocyte activation in silica-exposed workers. Int J Hyg Environ Health, 2014. 217(4-5): p. 586-91.

53. Rocha, M.C., et al., Genetic polymorphisms and surface expression of CTLA-4 and PD-1 on T cells of silica-exposed workers. International Journal of Hygiene and Environmental Health, 2012. 215(6): p. 562-569.

54. Rees, D. and J. Murray, Silica, silicosis and tuberculosis. Int J Tuberc Lung Dis, 2007. 11(5): p. 47484.

55. Bailey, W.C., et al., Silico-mycobacterial disease in sandblasters. Am Rev Respir Dis, 1974. 110(2): p. 115-25.

56. Shufflebotham, F., The Milroy Lectures on the hygienic aspect of the coal-mining industry in the United Kingdom: Delivered before the Royal College of Physicians of London. Br Med J, 1914. 1(2779): p. 755-9.

57. Hale, L.W., Tin mining and silicosis in Cornwall. Thorax, 1946. 1: p. 71-92.

58. Hnizdo, E. and J. Murray, Risk of pulmonary tuberculosis relative to silicosis and exposure to silica dust in South African gold miners. Occup Environ Med, 1998. 55(7): p. 496-502.

59. Cowie, R.L., The epidemiology of tuberculosis in gold miners with silicosis. Am J Respir Crit Care Med, 1994. 150(5 Pt 1): p. 1460-2.

60. teWaternaude, J.M., et al., Tuberculosis and silica exposure in South African gold miners. Occup Environ Med, 2006. 63(3): p. 187-92.

61. Barboza, C.E., et al., Tuberculosis and silicosis: epidemiology, diagnosis and chemoprophylaxis. J Bras Pneumol, 2008. 34(11): p. 959-66.

62. Sherson, D. and F. Lander, Morbidity of pulmonary tuberculosis among silicotic and nonsilicotic foundry workers in Denmark. J Occup Med, 1990. 32(2): p. 110-3.

63. Schaefer, W.B., et al., Infection with the avian-Battey group of mycobacteria in England and Wales. Br Med J, 1969. 2(5654): p. 412-5.

64. Wolinsky, E., Nontuberculous mycobacteria and associated diseases. Am Rev Respir Dis, 1979. 119(1): p. 107-59. 
65. Suzuki, S., et al., Clinical characteristics of pulmonary Mycobacterium scrofulaceum disease in 2001-2011: A case series and literature review. J Infect Chemother, 2016. 22(9): p. 611-6.

66. Kurahara, Y., et al., Mixed Pulmonary Infection with Three Types of Nontuberculous Mycobacteria. Internal Medicine, 2013. 52(4): p. 507-510.

67. Cordes, L.G., et al., A cluster of Acinetobacter Pneumonia in foundry workers. Ann Intern Med, 1981. 95(6): p. 688-93.

68. da Silva, G.A., et al., Cryptococcosis, silicosis, and tuberculous pseudotumor in the same pulmonary lobe. Jornal Brasileiro de Pneumologia : Publicaça̋o Oficial da Sociedade Brasileira de Pneumologia e Tisilogia, 2013. 39(5): p. 620-626.

69. Subra, J.F., et al., Lymphopenia in occupational pulmonary silicosis with or without autoimmune disease. Clin Exp Immunol, 2001. 126(3): p. 540-4.

70. Schuyler, M., M. Ziskind, and J. Salvaggio, Cell-mediated immunity in silicosis. Am Rev Respir Dis, 1977. 116(1): p. 147-51.

71. Schapira, R.M., et al., Hydroxyl radical production and lung injury in the rat following silica or titanium dioxide instillation in vivo. Am J Respir Cell Mol Biol, 1995. 12(2): p. 220-6.

72. Schapira, R.M., et al., L-arginine uptake and metabolism by lung macrophages and neutrophils following intratracheal instillation of silica in vivo. Am J Respir Cell Mol Biol, 1998. 19(2): p. 30815.

73. Porter, D.W., et al., Enhanced nitric oxide and reactive oxygen species production and damage after inhalation of silica. Am J Physiol Lung Cell Mol Physiol, 2002. 283(2): p. L485-93.

74. Porter, D.W., et al., Progression of lung inflammation and damage in rats after cessation of silica inhalation. Toxicol Sci, 2004. 79(2): p. 370-80.

75. Oberdorster, G., Toxicokinetics and effects of fibrous and nonfibrous particles. Inhal Toxicol, 2002. 14(1): p. 29-56.

76. Porter, D.W., et al., Nitric oxide and reactive oxygen species production causes progressive damage in rats after cessation of silica inhalation. Toxicol Sci, 2006. 90(1): p. 188-97.

77. Sato, T., et al., Suppressive Oligodeoxynucleotides Inhibit Silica-Induced Pulmonary Inflammation. The Journal of Immunology, 2008. 180(11): p. 7648-7654.

78. Schreck, R., P. Rieber, and P.A. Baeuerle, Reactive oxygen intermediates as apparently widely used messengers in the activation of the NF-kappa B transcription factor and HIV-1. The EMBO Journal, 1991. 10(8): p. 2247-2258.

79. Sen, C.K. and L. Packer, Antioxidant and redox regulation of gene transcription. Faseb j, 1996. 10(7): p. 709-20.

80. Porter, D.W., et al., Time course of pulmonary response of rats to inhalation of crystalline silica: NF-kappa B activation, inflammation, cytokine production, and damage. Inhal Toxicol, 2002. 14(4): p. 349-67.

81. Castranova, V., et al., Effect of inhaled crystalline silica in a rat model: Time course of pulmonary reactions. Molecular and Cellular Biochemistry, 2002. 234(1): p. 177-184.

82. Zhu, Z., et al., Suppression of thioredoxin system contributes to silica-induced oxidative stress and pulmonary fibrogenesis in rats. Toxicol Lett, 2013. 222(3): p. 289-94.

83. Peeters, P.M., et al., Silica-induced NLRP3 inflammasome activation in vitro and in rat lungs. Particle and Fibre Toxicology, 2014. 11(1): p. 58.

84. Ghio, A.J., R.H. Jaskot, and G.E. Hatch, Lung injury after silica instillation is associated with an accumulation of iron in rats. Am J Physiol, 1994. 267(6 Pt 1): p. L686-92.

85. Shimizu, Y., et al., Co-localization of iron binding on silica with p62/sequestosome1 (SQSTM1) in lung granulomas of mice with acute silicosis. Journal of Clinical Biochemistry and Nutrition, 2015. 56(1): p. 74-83. 
86. Jessop, F., et al., Autophagy deficiency in macrophages enhances NLRP3 inflammasome activity and chronic lung disease following silica exposure. Toxicol Appl Pharmacol, 2016. 309: p. 101-10.

87. Moncao-Ribeiro, L.C., et al., P2X7 receptor modulates inflammatory and functional pulmonary changes induced by silica. PLoS One, 2014. 9(10): p. e110185.

88. Wang, L., et al., Essential role of p53 in silica-induced apoptosis. Am J Physiol Lung Cell Mol Physiol, 2005. 288(3): p. L488-96.

89. Borges, V.M., et al., FAS Ligand Triggers Pulmonary Silicosis. The Journal of Experimental Medicine, 2001. 194(2): p. 155-164.

90. Suzuki, N., et al., T lymphocytes and silica-induced pulmonary inflammation and fibrosis in mice. Thorax, 1996. 51(10): p. 1036-42.

91. Langley, R.J., et al., A biphasic response to silica: I. Immunostimulation is restricted to the early stage of silicosis in lewis rats. Am J Respir Cell Mol Biol, 2004. 30(6): p. 823-9.

92. Migliaccio, C.T., R.F. Hamilton, Jr., and A. Holian, Increase in a distinct pulmonary macrophage subset possessing an antigen-presenting cell phenotype and in vitro APC activity following silica exposure. Toxicol Appl Pharmacol, 2005. 205(2): p. 168-76.

93. Liu, F., et al., CD4+CD25+Foxp3+ Regulatory T Cells Depletion May Attenuate the Development of Silica-Induced Lung Fibrosis in Mice. PLOS ONE, 2010. 5(11): p. e15404.

94. Guo, J., et al., Neutralization of interleukin-1 beta attenuates silica-induced lung inflammation and fibrosis in C57BL/6 mice. Arch Toxicol, 2013. 87.

95. Chen, Y., et al., Neutralization of interleukin-17A delays progression of silica-induced lung inflammation and fibrosis in C57BL/6 mice. Toxicol Appl Pharmacol, 2014. 275(1): p. 62-72.

96. Li, C., et al., Blocking the 4-1BB Pathway Ameliorates Crystalline Silica-induced Lung Inflammation and Fibrosis in Mice. Theranostics, 2016. 6(12): p. 2052-2067.

97. Wright, J.L., et al., Quartz But Not Iron Oxide Causes Air-Flow Obstruction, Emphysema, and Small Airways Lesions in the Rat. American Review of Respiratory Disease, 1988. 138(1): p. 129135.

98. Li, K., B. Keeling, and A. Churg, Mineral dusts cause elastin and collagen breakdown in the rat lung: a potential mechanism of dust-induced emphysema. Am J Respir Crit Care Med, 1996. 153(2): p. 644-9.

99. Churg, A., J. Hobson, and J. Wright, Functional and morphologic comparison of silica- and elastase-induced airflow obstruction. Exp Lung Res, 1989. 15(6): p. 813-22.

100. Herget, J., et al., Pulmonary Hypertension in Silicotic Rats. Archives of Environmental Health: An International Journal, 1979. 34(5): p. 320-324.

101. Zelko, I.N., et al., Pulmonary hypertension and vascular remodeling in mice exposed to crystalline silica. Respir Res, 2016. 17(1): p. 160.

102. Guo, J., et al., Effects of silica exposure on the cardiac and renal inflammatory and fibrotic response and the antagonistic role of interleukin-1 beta in C57BL/6 mice. Archives of Toxicology, 2016. 90(2): p. 247-258.

103. Brown, J.M., et al., Silica accelerated systemic autoimmune disease in lupus-prone New Zealand mixed mice. Clin Exp Immunol, 2003. 131(3): p. 415-21.

104. Brown, J.M., J.C. Pfau, and A. Holian, Immunoglobulin and Lymphocyte Responses Following Silica Exposure in New Zealand Mixed Mice. Inhalation Toxicology, 2004. 16(3): p. 133-139.

105. Pfau, J.C., J.M. Brown, and A. Holian, Silica-exposed mice generate autoantibodies to apoptotic cells. Toxicology, 2004. 195(2): p. 167-176.

106. Brown, J.M., et al., Effects of rottlerin on silica-exacerbated systemic autoimmune disease in New Zealand mixed mice. Am J Physiol Lung Cell Mol Physiol, 2005. 289(6): p. L990-8.

107. Strickland, F.M., et al., CD4(+) T cells epigenetically modified by oxidative stress cause lupus-like autoimmunity in mice. Journal of autoimmunity, 2015. 62: p. 75-80. 
108. Bates, M.A., et al., Silica Triggers Inflammation and Ectopic Lymphoid Neogenesis in the Lungs in Parallel with Accelerated Onset of Systemic Autoimmunity and Glomerulonephritis in the LupusProne NZBWF1 Mouse. PLOS ONE, 2015. 10(5): p. e0125481.

109. Bates, M.A., et al., Silica-Triggered Autoimmunity in Lupus-Prone Mice Blocked by Docosahexaenoic Acid Consumption. PLOS ONE, 2016. 11(8): p. e0160622.

110. Pasula, R., et al., Airway Delivery of Silica Increases Susceptibility to Mycobacterial Infection in Mice: Potential Role of Repopulating Macrophages. The Journal of Immunology, 2009. 182(11): p. 7102-7109.

111. Higgins, D.A., I.T. Kung, and R.S. Or, Environmental silica in badger lungs: a possible association with susceptibility to Mycobacterium bovis infection. Infect Immun, 1985. 48(1): p. 252-6.

112. Hatch, G.E., et al., Inhalable particles and pulmonary host defense: In vivo and in vitro effects of ambient air and combustion particles. Environmental Research, 1985. 36(1): p. 67-80.

113. Antonini, J.M., et al., Effect of silica inhalation on the pulmonary clearance of a bacterial pathogen in Fischer 344 rats. Lung, 2000. 178(6): p. 341-50.

114. Barbarin, V., et al., The role of pro- and anti-inflammatory responses in silica-induced lung fibrosis. Respir Res, 2005. 6: p. 112.

115. Huaux, F., Contribution of macrophage derived cytokines in the pathogenesis of lung fibrosis induced by inhaled mineral particles. 1998, Universite Catholique de Louvain.

116. Dörger, M., et al., Species differences in NO formation by rat and hamster alveolar macrophages in vitro. American Journal of Respiratory Cell and Molecular Biology, 1997. 16(4): p. 413-420.

117. Carter, J.M. and K.E. Driscoll, The role of inflammation, oxidative stress, and proliferation in silica-induced lung disease: a species comparison. J Environ Pathol Toxicol Oncol, 2001. 20 Suppl 1: p. 33-43.

118. Hatch, G.E., J.A. Raub, and J.A. Graham, Functional and biochemical indicators of pneumoconiosis in mice: comparison with rats. J Toxicol Environ Health, 1984. 13(4-6): p. 48797.

119. Liu, X., et al., Enhancement of proinflammatory and procoagulant responses to silica particles by monocyte-endothelial cell interactions. Particle and Fibre Toxicology, 2012. 9: p. 36-36.

120. Kobzik, L., Lung macrophage uptake of unopsonized environmental particulates. Role of scavenger-type receptors. J Immunol, 1995. 155(1): p. 367-76.

121. Allison, A.C., J.S. Harington, and M. Birbeck, An examination of the cytotoxic effects of silica on macrophages. J Exp Med, 1966. 124(2): p. 141-54.

122. Ding, M., et al., Diseases caused by silica: mechanisms of injury and disease development. Int Immunopharmacol, 2002. 2(2-3): p. 173-82.

123. Cassel, S.L., et al., The Nalp3 inflammasome is essential for the development of silicosis. Proc Natl Acad Sci U S A, 2008. 105(26): p. 9035-40.

124. Rom, W.N., Relationship of inflammatory cell cytokines to disease severity in individuals with occupational inorganic dust exposure. American Journal of Industrial Medicine, 1991. 19(1): p. 15-27.

125. Driscoll, K.E., et al., Macrophage Inflammatory Proteins 1 and 2: Expression by Rat Alveolar Macrophages, Fibroblasts, and Epithelial Cells and in Rat Lung after Mineral Dust Exposure. American Journal of Respiratory Cell and Molecular Biology, 1993. 8(3): p. 311-318.

126. Lugano, E.M., J.H. Dauber, and R.P. Daniele, Acute experimental silicosis. Lung morphology, histology, and macrophage chemotaxin secretion. Am J Pathol, 1982. 109(1): p. 27-36.

127. Huaux, F., New developments in the understanding of immunology in silicosis. Curr Opin Allergy Clin Immunol, 2007. 7(2): p. 168-73.

128. Kawasaki, H., A mechanistic review of silica-induced inhalation toxicity. Inhal Toxicol, 2015. 27(8): p. 363-77. 
129. Hornung, V., et al., Silica crystals and aluminum salts activate the NALP3 inflammasome through phagosomal destabilization. Nat Immunol, 2008. 9(8): p. 847-56.

130. Fubini, B. and A. Hubbard, Reactive oxygen species (ROS) and reactive nitrogen species (RNS) generation by silica in inflammation and fibrosis. Free Radic Biol Med, 2003. 34(12): p. 1507-16.

131. Iyer, R., et al., Silica-induced apoptosis mediated via scavenger receptor in human alveolar macrophages. Toxicol Appl Pharmacol, 1996. 141(1): p. 84-92.

132. Nash, T., A.C. Allison, and J.S. Harington, Physico-chemical properties of silica in relation to its toxicity. Nature, 1966. 210(5033): p. 259-61.

133. Shi, X.L., N.S. Dalal, and V. Vallyathan, ESR evidence for the hydroxyl radical formation in aqueous suspension of quartz particles and its possible significance to lipid peroxidation in silicosis. J Toxicol Environ Health, 1988. 25(2): p. 237-45.

134. Dalal, N.S., X.L. Shi, and V. Vallyathan, Role of free radicals in the mechanisms of hemolysis and lipid peroxidation by silica: comparative ESR and cytotoxicity studies. J Toxicol Environ Health, 1990. 29(3): p. 307-16.

135. Castranova, V., Generation of oxygen radicals and mechanisms of injury prevention. Environmental Health Perspectives, 1994. 102(Suppl 10): p. 65-68.

136. Vallyathan, V., et al., Response of alveolar macrophages to in vitro exposure to freshly fractured versus aged silica dust: the ability of Prosil 28, an organosilane material, to coat silica and reduce its biological reactivity. J Toxicol Environ Health, 1991. 33(3): p. 303-15.

137. Begin, R., et al., Aluminum lactate treatment alters the lung biological activity of quartz. Exp Lung Res, 1986. 10(4): p. 385-99.

138. Antonini, J.M. and M.J. Reasor, Effect of short-term exogenous pulmonary surfactant treatment on acute lung damage associated with the intratracheal instillation of silica. J Toxicol Environ Health, 1994. 43(1): p. 85-101.

139. Liu, X., et al., Phospholipid surfactant adsorption by respirable quartz and in vitro expression of cytotoxicity and DNA damage. Toxicol Lett, 1998. 96-97: p. 77-84.

140. Babior, B.M., The respiratory burst of phagocytes. J Clin Invest, 1984. 73(3): p. 599-601.

141. Badwey, J.A. and M.L. Karnovsky, Active oxygen species and the functions of phagocytic leukocytes. Annu Rev Biochem, 1980. 49: p. 695-726.

142. Zhang, Z., et al., Involvement of oxidative stress in crystalline silica-induced cytotoxicity and genotoxicity in rat alveolar macrophages. Environ Res, 2000. 82(3): p. 245-52.

143. Mintz, S., The effects of silica on the free, apparent redox state (NAD+/NADH) of the alveolar macrophage; a possible mitochondrial membrane effect. Chest, 1976. 69(2 Suppl): p. 298.

144. Fazzi, F., et al., TNFR1/Phox Interaction and TNFR1 Mitochondrial Translocation Thwart SilicaInduced Pulmonary Fibrosis. The Journal of Immunology, 2014. 192(8): p. 3837-3846.

145. Driscoll, K.E., et al., Effects of particle exposure and particle-elicited inflammatory cells on mutation in rat alveolar epithelial cells. Carcinogenesis, 1997. 18(2): p. 423-30.

146. Vallyathan, V., et al., Enhanced Generation of Free Radicals from Phagocytes Induced by Mineral Dusts. American Journal of Respiratory Cell and Molecular Biology, 1992. 6(4): p. 404-413.

147. Fubini, B., Surface Chemistry and Quartz Hazard. The Annals of Occupational Hygiene, 1998. 42(8): p. 521-530.

148. Pricop, L., et al., Reactive oxygen intermediates enhance Fc gamma receptor signaling and amplify phagocytic capacity. J Immunol, 1999. 162(12): p. 7041-8.

149. Ghio, A.J., et al., Role of surface complexed iron in oxidant generation and lung inflammation induced by silicates. Am J Physiol, 1992. 263(5 Pt 1): p. L511-8.

150. Kim, Y.K., et al., Depressant effect of ambroxol on stimulated functional responses and cell death in rat alveolar macrophages exposed to silica in vitro. J Pharmacol Exp Ther, 2002. 300(2): p. 629-37. 
151. Vallyathan, V., et al., Molecular Activation of Activator Protein-1 In Silica and Asbestos-Induced Carcinogenesis. Inhalation Toxicology, 2000. 12(sup3): p. 353-357.

152. Gwinn, M.R., et al., The Role of p53 in Silica-Induced Cellular and Molecular Responses Associated with Carcinogenesis. Journal of Toxicology and Environmental Health, Part A, 2009. 72(23): p. 1509-1519.

153. Naik, E. and V.M. Dixit, Mitochondrial reactive oxygen species drive proinflammatory cytokine production. The Journal of Experimental Medicine, 2011. 208(3): p. 417-420.

154. Abais, J.M., et al., Redox Regulation of NLRP3 Inflammasomes: ROS as Trigger or Effector? Antioxidants \& Redox Signaling, 2015. 22(13): p. 1111-1129.

155. Dostert, C., et al., Innate immune activation through Nalp3 inflammasome sensing of asbestos and silica. Science, 2008. 320.

156. Petrilli, V., et al., The inflammasome: a danger sensing complex triggering innate immunity. Curr Opin Immunol, 2007. 19(6): p. 615-22.

157. Dörger, M. and F. Krombach, Response of Alveolar Macrophages to Inhaled Particulates. European Surgical Research, 2002. 34(1-2): p. 47-52.

158. Beamer, C.A. and A. Holian, Silica suppresses Toll-like receptor ligand-induced dendritic cell activation. Faseb j, 2008. 22(6): p. 2053-63.

159. Lo Re, S., et al., IL-17A-Producing $\gamma \delta$ T and Th17 Lymphocytes Mediate Lung Inflammation but Not Fibrosis in Experimental Silicosis. The Journal of Immunology, 2010. 184(11): p. 6367-6377.

160. van Berlo, D., et al., NF-KB dependent and independent mechanisms of quartz-induced proinflammatory activation of lung epithelial cells. Particle and Fibre Toxicology, 2010. 7: p. 1313.

161. Goto, M., et al., Involvement of NF-kappaB p50/p65 heterodimer in activation of the human prointerleukin-1beta gene at two subregions of the upstream enhancer element. Cytokine, 1999. 11(1): p. 16-28.

162. Chen, F., et al., Essential role of NF-kappa B activation in silica-induced inflammatory mediator production in macrophages. Biochem Biophys Res Commun, 1995. 214(3): p. 985-92.

163. Hughes, C.S., et al., Extracellular cathepsin $S$ and intracellular caspase 1 activation are surrogate biomarkers of particulate-induced lysosomal disruption in macrophages. Part Fibre Toxicol, 2016. 13: p. 19.

164. Riteau, N., et al., ATP release and purinergic signaling: a common pathway for particle-mediated inflammasome activation. Cell Death \& Disease, 2012. 3(10).

165. Tsugita, M., et al., SR-B1 Is a Silica Receptor that Mediates Canonical Inflammasome Activation. Cell Rep, 2017. 18(5): p. 1298-1311.

166. Migliaccio, C.T., et al., The IL-4Ralpha pathway in macrophages and its potential role in silicainduced pulmonary fibrosis. J Leukoc Biol, 2008. 83(3): p. 630-9.

167. Rom, W.N., et al., Characterization of the lower respiratory tract inflammation of nonsmoking individuals with interstitial lung disease associated with chronic inhalation of inorganic dusts. Am Rev Respir Dis, 1987. 136(6): p. 1429-34.

168. Koren, H.S., et al., Modulation of eicosanoid production by human alveolar macrophages exposed to silica in vitro. Environ Health Perspect, 1992. 97: p. 77-83.

169. Barbarin, V., et al., Characterization of the effect of interleukin-10 on silica-induced lung fibrosis in mice. Am J Respir Cell Mol Biol, 2004. 31(1): p. 78-85.

170. Antognelli, C., et al., Glyoxalase I drives epithelial-to-mesenchymal transition via argpyrimidinemodified Hsp70, miR-21 and SMAD signalling in human bronchial cells BEAS-2B chronically exposed to crystalline silica Min-U-Sil 5: Transformation into a neoplastic-like phenotype. Free Radical Biology and Medicine, 2016. 92: p. 110-125. 
171. Ji, X., et al., IL4 and IL-17A provide a Th2/Th17-polarized inflammatory milieu in favor of TGF-B(1) to induce bronchial epithelial-mesenchymal transition (EMT). International Journal of Clinical and Experimental Pathology, 2013. 6(8): p. 1481-1492.

172. Mi, S., et al., Blocking IL-17A Promotes the Resolution of Pulmonary Inflammation and Fibrosis Via TGF-81-Dependent and-Independent Mechanisms. The Journal of Immunology, 2011. 187(6): p. 3003-3014.

173. Lo Re, S., et al., Platelet-derived growth factor-producing CD4+ Foxp3+ regulatory T lymphocytes promote lung fibrosis. Am J Respir Crit Care Med, 2011. 184(11): p. 1270-81.

174. Lee, S., et al., Silicosis and autoimmunity. Current Opinion in Allergy and Clinical Immunology, 2017. 17(2): p. 78-84.

175. Rao, T.D. and A.B. Frey, Administration of silica sensitizes lipopolysaccharide responsiveness of murine macrophages but inhibits $T$ and $B$ cell priming by inhibition of antigen presenting function. Immunol Invest, 1998. 27(3): p. 181-99.

176. Wu, P., et al., Induction of CD69 antigen expression in peripheral blood mononuclear cells on exposure to silica, but not by asbestos/chrysotile-A. Immunology Letters, 2005. 98(1): p. 145152.

177. Holian, A., et al., Asbestos and silica-induced changes in human alveolar macrophage phenotype. Environ Health Perspect, 1997. 105 Suppl 5: p. 1139-42.

178. Allison, A.C. and P.D. Hart, Potentiation by silica of the growth of Mycobacterium tuberculosis in macrophage cultures. Br J Exp Pathol, 1968. 49(5): p. 465-76.

179. QDNRM, Guidance note for management of diesel engine exhuast in metalliferous mines, in QGN 21, D.o.N.R.a.M. State of Queensland, Editor. 2014, Queensland Government, Australia: Mining and Quarrying Safety and Health Act of 1999. p. 1-26.

180. HSE, Control of diesel engine exhaust emissions in the workplace, in HSG187, H.a.S. Executive, Editor. 2012, Crown Copyright: Online. p. 1-21.

181. SUVA, D.o.O.M., Grenzwerte am Arbeitsplatz, in 1903.d, S.N.A.I.O. (SUVA), Editor. 2013, SUVA: online. p. 1-150.

182. OSHA/MSHA, Diesel Exhaust/Diesel Particulate Matter, in Hazard Alert. 2013, OSHA/MSHA: United States Department of Labor. p. 4.

183. TERA, Occupational Exposure Limit Evaluation: Diesel Particulate Matter, P. Nance, Editor. 2014, Toxicology Excellence for Risk Assessment: online. p. 1-15.

184. CDHS, Diesel Engine Exhaust Health Hazard Advisory, C.D.o.P. Health, Editor. 2002, Hazard Evaluation System and Information Service Health Hazard Advisory California Department of Public Health. p. 1-6.

185. Finkelstein, M.M., et al., Ischemic heart disease mortality among heavy equipment operators. American Journal of Industrial Medicine, 2004. 46(1): p. 16-22.

186. Hart, J.E., et al., Ischaemic heart disease mortality and years of work in trucking industry workers. Occup Environ Med, 2013. 70(8): p. 523-8.

187. Laden, F., et al., Cause-Specific Mortality in the Unionized U.S. Trucking Industry. Environmental Health Perspectives, 2007. 115(8): p. 1192-1196.

188. Torén, K., et al., Occupational exposure to particulate air pollution and mortality due to ischaemic heart disease and cerebrovascular disease. Occupational and Environmental Medicine, 2007. 64(8): p. 515-519.

189. Peters, A., et al., Increased particulate air pollution and the triggering of myocardial infarction. Circulation, 2001. 103(23): p. 2810-5.

190. Peng, R.D., et al., Emergency admissions for cardiovascular and respiratory diseases and the chemical composition of fine particle air pollution. Environ Health Perspect, 2009. 117(6): p. 95763. 
191. Pope, C.A., 3rd, et al., Ischemic heart disease events triggered by short-term exposure to fine particulate air pollution. Circulation, 2006. 114(23): p. 2443-8.

192. Yorifuji, T., S. Kashima, and H. Doi, Fine-particulate Air Pollution from Diesel Emission Control and Mortality Rates in Tokyo: A Quasi-experimental Study. Epidemiology, 2016. 27(6): p. 769-78.

193. Latifovic, L., et al., Bladder cancer and occupational exposure to diesel and gasoline engine emissions among Canadian men. Cancer Med, 2015. 4(12): p. 1948-62.

194. Seidler, A., et al., Association between diesel exposure at work and prostate cancer. Scandinavian journal of work, environment \& health, 1998. 24(6): p. 486-94.

195. Guo, J., et al., Risk of esophageal, ovarian, testicular, kidney and bladder cancers and leukemia among finnish workers exposed to diesel or gasoline engine exhaust. Int J Cancer, 2004. 111(2): p. 286-92.

196. Bakke, B., et al., Cumulative exposure to dust and gases as determinants of lung function decline in tunnel construction workers. Occup Environ Med, 2004. 61(3): p. 262-9.

197. Silverman, D.T., et al., The Diesel Exhaust in Miners Study: A Nested Case-Control Study of Lung Cancer and Diesel Exhaust. JNCl Journal of the National Cancer Institute, 2012. 104(11): p. 855868.

198. Ulfvarson, U. and R. Alexandersson, Reduction in adverse effect on pulmonary function after exposure to filtered diesel exhaust. Am J Ind Med, 1990. 17(3): p. 341-7.

199. Chattopadhyay, B.P., J. Alam, and A. Roychowdhury, Pulmonary Function Abnormalities Associated with Exposure to Automobile Exhaust in a Diesel Bus Garage and Roads. Lung, 2003. 181(5): p. 291-302.

200. Gamble, J.F. and W.G. Jones, Respiratory Effects of Diesel Exhaust in Salt Miners. American Review of Respiratory Disease, 1983. 128(3): p. 389-394.

201. Gamble, J., W. Jones, and J. Hudak, An epidemiological study of salt miners in diesel and nondiesel mines. Am J Ind Med, 1983. 4(3): p. 435-58.

202. Gamble, J., W. Jones, and S. Minshall, Epidemiological-environmental study of diesel bus garage workers: Chronic effects of diesel exhaust on the respiratory system. Environmental Research, 1987. 44(1): p. 6-17.

203. Zhang, L.P., et al., Long-term exposure to diesel engine exhaust induced lung function decline in a cross sectional study. Ind Health, 2017. 55(1): p. 13-26.

204. Ulvestad, B., et al., Increased risk of obstructive pulmonary disease in tunnel workers. Thorax, 2000. 55(4): p. 277-282.

205. Bergdahl, I.A., et al., Increased mortality in COPD among construction workers exposed to inorganic dust. European Respiratory Journal, 2004. 23(3): p. 402-406.

206. Hart, J.E., et al., Chronic obstructive pulmonary disease mortality in railroad workers. Occupational and environmental medicine, 2009. 66(4): p. 221-226.

207. Hart, J.E., et al., Chronic obstructive pulmonary disease mortality in diesel-exposed railroad workers. Environ Health Perspect, 2006. 114(7): p. 1013-7.

208. Weinmann, S., et al., COPD and Occupational Exposures: A Case-Control Study. Journal of Occupational and Environmental Medicine, 2008. 50(5): p. 561-569.

209. Jorgensen, H. and A. Svensson, Studies on Pulmonary Function and Respiratory Tract Symptoms of Workers in an Iron Ore Mine Where Diesel Trucks are Used Underground. Journal of Occupational and Environmental Medicine, 1970. 12(9): p. 348-354.

210. Oliver, L.C., et al., Respiratory symptoms and lung function in workers in heavy and highway construction: A cross-sectional study*. American Journal of Industrial Medicine, 2001. 40(1): p. 73-86. 
211. Gluck, U., R. Schutz, and J.O. Gebbers, Cytopathology of the nasal mucosa in chronic exposure to diesel engine emission: a five-year survey of Swiss customs officers. Environ Health Perspect, 2003. 111(7): p. 925-9.

212. Safak, A.A., et al., Bronchial wall thickness in toll collectors. Ind Health, 2010. 48(3): p. 317-23.

213. Wade, J.F., 3rd and L.S. Newman, Diesel asthma. Reactive airways disease following overexposure to locomotive exhaust. J Occup Med, 1993. 35(2): p. 149-54.

214. Adewole, F., et al., Diesel exhaust causing low-dose irritant asthma with latency? Occup Med (Lond), 2009. 59(6): p. 424-7.

215. IARC, Diesel and Gasoline Engine Exhausts and Some Nitroarenes. IARC Monographs on the Evaluation of Carcinogenic Risks to Humans. IARC Monogr Eval Carcinog Risks Hum, 2014. 105: p. 9-699.

216. Garshick, E., et al., Lung Cancer in Railroad Workers Exposed to Diesel Exhaust. Environmental Health Perspectives, 2004. 112(15): p. 1539-1543.

217. Boffetta, P., S.D. Stellman, and L. Garfinkel, Diesel exhaust exposure and mortality among males in the American cancer society prospective study. American Journal of Industrial Medicine, 1988. 14(4): p. 403-415.

218. Soll-Johanning, H., et al., Cancer incidence in urban bus drivers and tramway employees: a retrospective cohort study. Occup Environ Med, 1998. 55(9): p. 594-8.

219. Steenland, K., J. Deddens, and L. Stayner, Diesel exhaust and lung cancer in the trucking industry: exposure-response analyses and risk assessment. Am J Ind Med, 1998. 34(3): p. 220-8.

220. Brunekreef, B., et al., Air Pollution from Truck Traffic and Lung Function in Children Living near Motorways. Epidemiology, 1997. 8(3): p. 298-303.

221. Northridge, M.E., et al., Diesel exhaust exposure among adolescents in Harlem: a communitydriven study. Am J Public Health, 1999. 89(7): p. 998-1002.

222. Margolis, H.G., et al., Altered pulmonary function in children with asthma associated with highway traffic near residence. Int J Environ Health Res, 2009. 19(2): p. 139-55.

223. Morgenstern, V., et al., Atopic Diseases, Allergic Sensitization, and Exposure to Traffic-related Air Pollution in Children. American Journal of Respiratory and Critical Care Medicine, 2008. 177(12): p. 1331-1337.

224. Lindgren, A., et al., Traffic-related air pollution associated with prevalence of asthma and COPD/chronic bronchitis. A cross-sectional study in Southern Sweden. International Journal of Health Geographics, 2009. 8(1): p. 2.

225. Beatty, T.K.M. and J.P. Shimshack, School buses, diesel emissions, and respiratory health. Journal of Health Economics, 2011. 30(5): p. 987-999.

226. Salameh, P., et al., Exposure to outdoor air pollution and chronic bronchitis in adults: a casecontrol study. Int J Occup Environ Med, 2012. 3(4): p. 165-77.

227. Schultz, A.A., J.J. Schauer, and K.M. Malecki, Allergic disease associations with regional and localized estimates of air pollution. Environmental research, 2017. 155: p. 77-85.

228. Wong, T.W., et al., Air pollution and hospital admissions for respiratory and cardiovascular diseases in Hong Kong. Occupational and Environmental Medicine, 1999. 56(10): p. 679-683.

229. McGowan, J.A., et al., Particulate air pollution and hospital admissions in Christchurch, New Zealand. Aust N Z J Public Health, 2002. 26(1): p. 23-9.

230. Neupane, B., et al., Long-term exposure to ambient air pollution and risk of hospitalization with community-acquired pneumonia in older adults. American journal of respiratory and critical care medicine, 2010. 181(1): p. 47-53.

231. Rudell, B., et al., Bronchoalveolar inflammation after exposure to diesel exhaust: comparison between unfiltered and particle trap filtered exhaust. Occupational and Environmental Medicine, 1999. 56(8): p. 527-534. 
232. Rudell, B., et al., Efficiency of automotive cabin air filters to reduce acute health effects of diesel exhaust in human subjects. Occupational and Environmental Medicine, 1999. 56(4): p. 222-231.

233. Carlsten, C., et al., Symptoms in Response to Controlled Diesel Exhaust More Closely Reflect Exposure Perception Than True Exposure. PLoS ONE, 2013. 8(12): p. e83573.

234. Nightingale, J.A., et al., Airway inflammation after controlled exposure to diesel exhaust particulates. Am J Respir Crit Care Med, 2000. 162(1): p. 161-6.

235. Salvi, S., et al., Acute inflammatory responses in the airways and peripheral blood after shortterm exposure to diesel exhaust in healthy human volunteers. American journal of respiratory and critical care medicine, 1999. 159(3): p. 702-9.

236. Behndig, A.F., et al., Airway antioxidant and inflammatory responses to diesel exhaust exposure in healthy humans. European Respiratory Journal, 2006. 27(2): p. 359-365.

237. Sehlstedt, M., et al. Airway inflammatory response to diesel exhaust generated at urban cycle running conditions. Inhalation Toxicology, 2010. 22, 1144-1150.

238. Barath, S., et al., Diesel exhaust but not ozone increases fraction of exhaled nitric oxide in a randomized controlled experimental exposure study of healthy human subjects. Environmental Health, 2013. 12: p. 36-36.

239. Bosson, J., et al., Ozone enhances the airway inflammation initiated by diesel exhaust. Respiratory Medicine, 2007. 101(6): p. 1140-1146.

240. Hayes, J.D., J.U. Flanagan, and I.R. Jowsey, Glutathione transferases. Annual review of pharmacology and toxicology, 2005. 45: p. 51-88.

241. Madden, M.C., et al., Diesel exhaust modulates ozone-induced lung function decrements in healthy human volunteers. Part Fibre Toxicol, 2014. 11: p. 37.

242. Miyamoto, T., Epidemiology of pollution-induced airwav disease in Japan. Allergy, 1997. 52: $\mathrm{p}$. 30-34.

243. Larsson, N., et al., Airway inflammatory responses to diesel exhaust in allergic rhinitics. Inhalation toxicology, 2013. 25(3): p. 160-7.

244. Stenfors, N., et al., Different airway inflammatory responses in asthmatic and healthy humans exposed to diesel. European Respiratory Journal, 2004. 23(1): p. 82-86.

245. Behndig, A.F., et al., Proinflammatory doses of diesel exhaust in healthy subjects fail to elicit equivalent or augmented airway inflammation in subjects with asthma. Thorax, 2011. 66(1): $p$. 12-19.

246. McCreanor, J., et al., Respiratory Effects of Exposure to Diesel Traffic in Persons with Asthma. New England Journal of Medicine, 2007. 357(23): p. 2348-2358.

247. Hosseini, A., et al., Morphometric analysis of inflammation in bronchial biopsies following exposure to inhaled diesel exhaust and allergen challenge in atopic subjects. Particle and Fibre Toxicology, 2015. 13: p. 2.

248. Frampton, M.W., et al., Nitrogen dioxide exposure in vivo and human alveolar macrophage inactivation of influenza virus in vitro. Environ Res, 1989. 48(2): p. 179-92.

249. Pawlak, E.A., et al., Diesel exposure suppresses natural killer cell function and resolution of eosinophil inflammation: a randomized controlled trial of exposure in allergic rhinitics. Part Fibre Toxicol, 2016. 13(1): p. 24.

250. Helleday, R., et al., Nitrogen dioxide exposure impairs the frequency of the mucociliary activity in healthy subjects. European Respiratory Journal, 1995. 8(10): p. 1664-1668.

251. Vallyathan, V., et al., Effect of diesel emissions and coal dust inhalation on heart and pulmonary arteries of rats. J Toxicol Environ Health, 1986. 19(1): p. 33-41.

252. Campen, M.J., et al., Nonparticulate components of diesel exhaust promote constriction in coronary arteries from ApoE-/- mice. Toxicol Sci, 2005. 88(1): p. 95-102. 
253. Campen, M.J., et al., Cardiovascular effects of inhaled diesel exhaust in spontaneously hypertensive rats. Cardiovasc Toxicol, 2003. 3(4): p. 353-61.

254. Hazari, M.S., et al., Dobutamine "stress" test and latent cardiac susceptibility to inhaled diesel exhaust in normal and hypertensive rats. Environ Health Perspect, 2012. 120(8): p. 1088-93.

255. Carll, A.P., et al., Whole and particle-free diesel exhausts differentially affect cardiac electrophysiology, blood pressure, and autonomic balance in heart failure-prone rats. Toxicol Sci, 2012. 128(2): p. 490-9.

256. Yokota, S., et al., Tracheal instillation of diesel exhaust particles component causes blood and pulmonary neutrophilia and enhances myocardial oxidative stress in mice. J Toxicol Sci, 2008. 33(5): p. 609-20.

257. Robertson, S., et al., Pulmonary diesel particulate increases susceptibility to myocardial ischemia/reperfusion injury via activation of sensory TRPV1 and beta1 adrenoreceptors. Part Fibre Toxicol, 2014. 11: p. 12.

258. Chen, S., M.A. Weller, and M.I. Barnhart, Effects of Diesel engine exhaust on pulmonary alveolar macrophages. Scan Electron Microsc, 1980(3): p. 327-38.

259. Chen, S., M.A. Weller, and M.I. Barnhart, Species comparisons of bronchoalveolar lavages from guinea pigs and rats exposed in vivo to diesel exhaust for one year. Scanning electron microscopy, 1982(Pt 4): p. 1687-98.

260. Pepelko, W.E., et al., Pulmonary function and pathology in cats exposed 28 days to diesel exhaust. J Environ Pathol Toxicol, 1980. 4(2-3): p. 449-57.

261. Abraham, W.M., et al., Effects of a brief low-level exposure to the particulate fraction of diesel exhaust on pulmonary function of conscious sheep. Arch Environ Health, 1980. 35(2): p. 77-80.

262. Gross, K.B., Pulmonary function testing of animals chronically exposed to diluted diesel exhaust for 267 days. Environment International, 1981. 5(4): p. 331-337.

263. Gross, K.B., Pulmonary function testing of animals chronically exposed to diluted diesel exhaust. J Appl Toxicol, 1981. 1(2): p. 116-23.

264. Moorman, W.J., et al., Pulmonary function responses in cats following long-term exposure to diesel exhaust. J Appl Toxicol, 1985. 5(5): p. 301-5.

265. Heinrich, U., et al., Chronic effects on the respiratory tract of hamsters, mice and rats after longterm inhalation of high concentrations of filtered and unfiltered diesel engine emissions. J Appl Toxicol, 1986. 6(6): p. 383-95.

266. Henderson, R.F., et al., Species differences in release of arachidonate metabolites in response to inhaled diluted diesel exhaust. Toxicology Letters, 1988. 42(3): p. 325-332.

267. Ulfvarson, U., et al., Experimental Evaluation of the Effect of Filtration of Diesel Exhaust by Biologic Exposure Indicators. American Journal of Industrial Medicine, 1995. 27(1): p. 91-106.

268. Inoue, K., et al., The role of toll-like receptor 4 in airway inflammation induced by diesel exhaust particles. Arch Toxicol, 2006. 80(5): p. 275-9.

269. Fujimaki, H., et al., Distinct requirements for interleukin-6 in airway inflammation induced by diesel exhaust in mice. Immunopharmacol Immunotoxicol, 2006. 28(4): p. 703-14.

270. Ahn, E.-K., et al., COX-2 expression and inflammatory effects by diesel exhaust particles in vitro and in vivo. Toxicology Letters, 2008. 176(3): p. 178-187.

271. Sagai, M., et al., Biological effects of diesel exhaust particles. I. in vitro production of superoxide and in vivo toxicity in mouse. Free Radical Biology and Medicine, 1993. 14(1): p. 37-47.

272. Lim, H.B., et al., Involvement of superoxide and nitric oxide on airway inflammation and hyperresponsiveness induced by diesel exhaust particles in mice. Free Radic Biol Med, 1998. 25(6): p. 635-44.

273. Ito, T., et al., Peroxynitrite formation by diesel exhaust particles in alveolar cells: Links to pulmonary inflammation. Environmental Toxicology and Pharmacology, 2000. 9(1): p. 1-8. 
274. Han, J.-Y., K. Takeshita, and H. Utsumi, Noninvasive detection of hydroxyl radical generation in lung by diesel exhaust particles. Free Radical Biology and Medicine, 2001. 30(5): p. 516-525.

275. Nemmar, A., et al., Emodin mitigates diesel exhaust particles-induced increase in airway resistance, inflammation and oxidative stress in mice. Respiratory Physiology \& Neurobiology, 2015. 215: p. 51-57.

276. Madden, M.C., et al., Effect of Ozone on Diesel Exhaust Particle Toxicity in Rat Lung. Toxicology and Applied Pharmacology, 2000. 168(2): p. 140-148.

277. Zhao, H., et al., Cooperation of the inducible nitric oxide synthase and cytochrome P450 1A1 in mediating lung inflammation and mutagenicity induced by diesel exhaust particles. Environ Health Perspect, 2006. 114(8): p. 1253-8.

278. Zhao, H., et al., Reactive oxygen species- and nitric oxide-mediated lung inflammation and mitochondrial dysfunction in wild-type and iNOS-deficient mice exposed to diesel exhaust particles. Journal of toxicology and environmental health. Part A, 2009. 72(8): p. 560-70.

279. Fedan, J.S., et al., Effects of a two-year inhalation exposure of rats to coal dust and/or diesel exhaust on tension responses of isolated airway smooth muscle. Am Rev Respir Dis, 1985. 131(4): p. 651-5.

280. Ishihara, Y. and J. Kagawa, Chronic diesel exhaust exposures of rats demonstrate concentration and time-dependent effects on pulmonary inflammation. Inhal Toxicol, 2003. 15(5): p. 473-92.

281. Laks, D., et al., Composition of diesel particles influences acute pulmonary toxicity: an experimental study in mice. Inhal Toxicol, 2008. 20(11): p. 1037-42.

282. Yoshizaki, K., et al., Subchronic effects of nasally instilled diesel exhaust particulates on the nasal and airway epithelia in mice. Inhalation Toxicology, 2010. 22(7): p. 610-617.

283. Shaheen, H.M., et al., The ceramide inhibitor fumonisin B1 mitigates the pulmonary effects of low-dose diesel exhaust inhalation in mice. Ecotoxicology and Environmental Safety, 2016. 132: p. 390-396.

284. Sagai, M. and T. Ichinose, [Role of nitric oxide in asthma-like symptoms induced by diesel exhaust particles in mice]. Nihon Kyobu Shikkan Gakkai Zasshi, 1995. 33 Suppl: p. 212-7.

285. Sagai, M., A. Furuyama, and T. Ichinose, Biological effects of diesel exhaust particles (DEP). III. Pathogenesis of asthma like symptoms in mice. Free Radical Biology and Medicine, 1996. 21(2): p. 199-209.

286. Aguilera-Aguirre, L., et al., Mitochondrial dysfunction increases allergic airway inflammation. J Immunol, 2009. 183(8): p. 5379-87.

287. Ohta, K., et al., Diesel exhaust particulate induces airway hyperresponsiveness in a murine model: Essential role of GM-CSF. Journal of Allergy and Clinical Immunology, 1999. 104(5): p. 1024-1030.

288. Yamashita, N., et al., Platelet-derived growth factor is involved in the augmentation of airway responsiveness through remodeling of airways in diesel exhaust particulate-treated mice. J Allergy Clin Immunol, 2001. 107(1): p. 135-42.

289. Brandt, E.B., et al., Diesel exhaust particle induction of IL-17A contributes to severe asthma. J Allergy Clin Immunol, 2013. 132(5): p. 1194-1204.e2.

290. Suzuki, T., et al., Adjuvant activity of diesel exhaust particulates (DEP) in production of anti-IgE and anti-IgG1 antibodies to mite allergen in mice. J Clin Lab Immunol, 1996. 48(5): p. 187-99.

291. Takano, H., et al., Diesel exhaust particles enhance antigen-induced airway inflammation and local cytokine expression in mice. Am J Respir Crit Care Med, 1997. 156(1): p. 36-42.

292. Miyabara, Y., et al., Effects of diesel exhaust on allergic airway inflammation in mice. Journal of Allergy and Clinical Immunology, 1998. 102(5): p. 805-812.

293. Steerenberg, P.A., et al., A pollen model in the rat for testing adjuvant activity of air pollution components. Inhal Toxicol, 1999. 11(12): p. 1109-22. 
294. Hao, M., et al., Diesel exhaust particles exert acute effects on airway inflammation and function in murine allergen provocation models. J Allergy Clin Immunol, 2003. 112(5): p. 905-14.

295. Al-Humadi, N.H., et al., The effect of diesel exhaust particles (DEP) and carbon black (CB) on thiol changes in pulmonary ovalbumin allergic sensitized Brown Norway rats. Exp Lung Res, 2002. 28(5): p. 333-49.

296. Inoue, K., et al., Effects of components derived from diesel exhaust particles on lung physiology related to antigen. Immunopharmacol Immunotoxicol, 2007. 29(3-4): p. 403-12.

297. Park, E.J., et al., Biological responses to diesel exhaust particles (DEPS) depend on the physicochemical properties of the DEPs. PLoS One, 2011. 6(10): p. e26749.

298. Tanaka, M., et al., Effects of exposure to nanoparticle-rich or-depleted diesel exhaust on allergic pathophysiology in the murine lung. J Toxicol Sci, 2013. 38(1): p. 35-48.

299. Stone, K.D., C. Prussin, and D.D. Metcalfe, IgE, mast cells, basophils, and eosinophils. J Allergy Clin Immunol, 2010. 125(2 Suppl 2): p. S73-80.

300. Jang, A.S., et al., Additive effect of diesel exhaust particulates and ozone on airway hyperresponsiveness and inflammation in a mouse model of asthma. J Korean Med Sci, 2005. 20(5): p. 759-63.

301. Liu, J., et al., Combined inhaled diesel exhaust particles and allergen exposure alter methylation of Thelper genes and IgE production in vivo. Toxicol Sci, 2008. 102(1): p. 76-81.

302. De Grove, K.C., et al., Dysregulation of type 2 innate lymphoid cells and TH2 cells impairs pollutant-induced allergic airway responses. J Allergy Clin Immunol, 2017. 139(1): p. 246-257.e4.

303. Kotin, P., H.L. Falk, and M. Thomas, Aromatic hydrocarbons. II. Presence in the particulate phase of gasoline-engine exhausts and the carcinogenicity of exhaust extracts. AMA Arch Ind Hyg Occup Med, 1954. 9(2): p. 164-77.

304. Kotin, P., H.L. Falk, and M. Thomas, Aromatic hydrocarbons. III. Presence in the particulate phase of diesel-engine exhausts and the carcinogenicity of exhaust extracts. AMA Arch Ind Health, 1955. 11(2): p. 113-20.

305. Oberdorster, G., Lung particle overload: implications for occupational exposures to particles. Regul Toxicol Pharmacol, 1995. 21(1): p. 123-35.

306. Hesterberg, T.W., et al., Carcinogenicity studies of diesel engine exhausts in laboratory animals: a review of past studies and a discussion of future research needs. Crit Rev Toxicol, 2005. 35(5): p. 379-411.

307. Heinrich, U., et al., Chronic Inhalation Exposure of Wistar Rats and two Different Strains of Mice to Diesel Engine Exhaust, Carbon Black, and Titanium Dioxide. Inhalation Toxicology, 1995. 7(4): p. 533-556.

308. Mauderly, J.L., et al., Pulmonary toxicity of inhaled diesel exhaust and carbon black in chronically exposed rats. Part I: Neoplastic and nonneoplastic lung lesions. Res Rep Health Eff Inst, 1994(68 Pt 1): p. 1-75; discussion 77-97.

309. Nikula, K.J., et al., Comparative Pulmonary Toxicities and Carcinogenicities of Chronically Inhaled Diesel Exhaust and Carbon Black in F344 Rats. Fundamental and Applied Toxicology, 1995. 25(1): p. 80-94.

310. Iwai, K., et al., Early oxidative DNA damages and late development of lung cancer in diesel exhaust-exposed rats. Environ Res, 2000. 84(3): p. 255-64.

311. Stinn, W., et al., Chronic Nose-only Inhalation Study in Rats, comparing Room-aged Sidestream Cigarette Smoke and Diesel Engine Exhaust. Inhalation Toxicology, 2005. 17(11): p. 549-576.

312. Harrod, K.S., et al., Increased susceptibility to RSV infection by exposure to inhaled diesel engine emissions. Am J Respir Cell Mol Biol, 2003. 28(4): p. 451-63.

313. Harrod, K.S., et al., Inhaled diesel engine emissions reduce bacterial clearance and exacerbate lung disease to Pseudomonas aeruginosa infection in vivo. Toxicol Sci, 2005. 83(1): p. 155-65. 
314. Ciencewicki, J., et al., Diesel exhaust enhanced susceptibility to influenza infection is associated with decreased surfactant protein expression. Inhal Toxicol, 2007. 19(14): p. 1121-33.

315. Gowdy, K., et al., Modulation of pulmonary inflammatory responses and antimicrobial defenses in mice exposed to diesel exhaust. Toxicol Appl Pharmacol, 2008. 229(3): p. 310-9.

316. Yin, X.J., et al., Alteration of pulmonary immunity to Listeria monocytogenes by diesel exhaust particles (DEPS). I. Effects of DEPs on early pulmonary responses. Environ Health Perspect, 2002. 110(11): p. 1105-11.

317. Yin, X.J., et al., Alteration of pulmonary immunity to Listeria monocytogenes by diesel exhaust particles (DEPS). II. Effects of DEPs on T-cell-mediated immune responses in rats. Environ Health Perspect, 2003. 111(4): p. 524-30.

318. Yin, X.J., et al., Sustained effect of inhaled diesel exhaust particles on T-lymphocyte-mediated immune responses against Listeria monocytogenes. Toxicol Sci, 2005. 88(1): p. 73-81.

319. Hiramatsu, K., et al., The effects of inhalation of diesel exhaust on murine mycobacterial infection. Exp Lung Res, 2005. 31(4): p. 405-15.

320. Saito, Y., et al., Long-term inhalation of diesel exhaust affects cytokine expression in murine lung tissues: comparison between low- and high-dose diesel exhaust exposure. Exp Lung Res, 2002. 28(6): p. 493-506.

321. Hahon, N., et al., Influenza virus infection in mice after exposure to coal dust and diesel engine emissions. Environ Res, 1985. 37(1): p. 44-60.

322. Gowdy, K.M., et al., Role of oxidative stress on diesel-enhanced influenza infection in mice. Part Fibre Toxicol, 2010. 7: p. 34.

323. Larcombe, A.N., et al., Acute diesel exhaust particle exposure increases viral titre and inflammation associated with existing influenza infection, but does not exacerbate deficits in lung function. Influenza Other Respir Viruses, 2013. 7(5): p. 701-9.

324. Yang, H.M., et al., Diesel exhaust particles suppress macrophage function and slow the pulmonary clearance of Listeria monocytogenes in rats. Environ Health Perspect, 2001. 109(5): p. 515-21.

325. Castranova, V., et al., Effect of exposure to diesel exhaust particles on the susceptibility of the lung to infection. Environ Health Perspect, 2001. 109 Suppl 4: p. 609-12.

326. Yin, X.J., et al., Roles of reactive oxygen species and heme oxygenase-1 in modulation of alveolar macrophage-mediated pulmonary immune responses to Listeria monocytogenes by diesel exhaust particles. Toxicol Sci, 2004. 82(1): p. 143-53.

327. Ma, J.Y. and J.K. Ma, The dual effect of the particulate and organic components of diesel exhaust particles on the alteration of pulmonary immune/inflammatory responses and metabolic enzymes. J Environ Sci Health C Environ Carcinog Ecotoxicol Rev, 2002. 20(2): p. 117-47.

328. Vogl, G. and E.F. Elstner, Diesel soot particles catalyze the production of oxy-radicals. Toxicol Lett, 1989. 47(1): p. 17-23.

329. Dong, C., Diesel exhaust particulate-mediated oxidative response by alveolar macrophages. 1998.

330. Shukla, A., et al., Inhaled particulate matter causes expression of nuclear factor (NF)-kappaBrelated genes and oxidant-dependent NF-kappaB activation in vitro. Am J Respir Cell Mol Biol, 2000. 23(2): p. 182-7.

331. Greenwell, L.L., et al., Particle-induced oxidative damage is ameliorated by pulmonary antioxidants. Free Radical Biology and Medicine, 2002. 32(9): p. 898-905.

332. Xiao, G.G., et al., Use of proteomics to demonstrate a hierarchical oxidative stress response to diesel exhaust particle chemicals in a macrophage cell line. J Biol Chem, 2003. 278(50): p. 5078190. 
333. Jung, E.J., et al., Pro-oxidative DEP chemicals induce heat shock proteins and an unfolding protein response in a bronchial epithelial cell line as determined by DIGE analysis. Proteomics, 2007. 7(21): p. 3906-18.

334. Hiura, T.S., et al., Chemicals in Diesel Exhaust Particles Generate Reactive Oxygen Radicals and Induce Apoptosis in Macrophages. The Journal of Immunology, 1999. 163(10): p. 5582-5591.

335. Hiura, T.S., et al., The role of a mitochondrial pathway in the induction of apoptosis by chemicals extracted from diesel exhaust particles. J Immunol, 2000. 165(5): p. 2703-11.

336. Bonvallot, V., et al., Activation of Transcription Factors by Diesel Exhaust Particles in Human Bronchial Epithelial Cells In Vitro. Inhalation Toxicology, 2000. 12(sup3): p. 359-364.

337. Kafoury, R.M. and J. Kelley, Ozone enhances diesel exhaust particles (DEP)-induced interleukin-8 (IL-8) gene expression in human airway epithelial cells through activation of nuclear factorskappaB (NF-kappaB) and IL-6 (NF-IL6). Int J Environ Res Public Health, 2005. 2(3-4): p. 403-10.

338. Kaimul Ahsan, M., et al., Thioredoxin-1 suppresses lung injury and apoptosis induced by diesel exhaust particles (DEP) by scavenging reactive oxygen species and by inhibiting DEP-induced downregulation of Akt. Free Radic Biol Med, 2005. 39(12): p. 1549-59.

339. Kafoury, R.M. and M.C. Madden, Diesel Exhaust Particles Induce the Over expression of Tumor Necrosis Factor- $\alpha$ (TNF- $\alpha$ ) Gene in Alveolar Macrophages and Failed to Induce Apoptosis through Activation of Nuclear Factor-KB (NF-KB). International Journal of Environmental Research and Public Health, 2005. 2(1): p. 107-113.

340. Park, S., et al., The role of iron in reactive oxygen species generation from diesel exhaust particles. Toxicology in Vitro, 2006. 20(6): p. 851-857.

341. DiStefano, E., et al., Determination of metal-based hydroxyl radical generating capacity of ambient and diesel exhaust particles. Inhalation toxicology, 2009. 21(9): p. 731-8.

342. Ghio, A.J., et al., Metals associated with both the water-soluble and insoluble fractions of an ambient air pollution particle catalyze an oxidative stress. Inhal Toxicol, 1999. 11(1): p. 37-49.

343. Ghio, A.J., M.S. Carraway, and M.C. Madden, Composition of air pollution particles and oxidative stress in cells, tissues, and living systems. J Toxicol Environ Health B Crit Rev, 2012. 15(1): p. 121.

344. Bayram, H., et al., Comparison of ciliary activity and inflammatory mediator release from bronchial epithelial cells of nonatopic nonasthmatic subjects and atopic asthmatic patients and the effect of diesel exhaust particles in vitro. Journal of Allergy and Clinical Immunology, 1998. 102(5): p. 771-782.

345. Segura-Valdez, L., et al., Upregulation of gelatinases $A$ and $B$, collagenases 1 and 2, and increased parenchymal cell death in COPD. Chest, 2000. 117(3): p. 684-94.

346. Li, J., et al., Diesel exhaust particles activate the matrix-metalloproteinase-1 gene in human bronchial epithelia in a beta-arrestin-dependent manner via activation of RAS. Environ Health Perspect, 2009. 117(3): p. 400-9.

347. Zhu, G., et al., Association of TRPV4 gene polymorphisms with chronic obstructive pulmonary disease. Hum Mol Genet, 2009. 18(11): p. 2053-62.

348. Li, J., et al., TRPV4-mediated calcium influx into human bronchial epithelia upon exposure to diesel exhaust particles. Environ Health Perspect, 2011. 119(6): p. 784-93.

349. Park, I.H., et al., Diesel Exhaust Particles Enhance MUC4 Expression in NCl-H292 Cells and Nasal Epithelial Cells via the p38/CREB Pathway. Int Arch Allergy Immunol, 2016. 171(3-4): p. 209-216.

350. Robinson, R.K., et al., Mechanistic link between diesel exhaust particles and respiratory reflexes. J Allergy Clin Immunol, 2017.

351. Terada, N., et al., Diesel exhaust particulates enhance eosinophil adhesion to nasal epithelial cells and cause degranulation. Int Arch Allergy Immunol, 1997. 114(2): p. 167-74. 
352. Terada, N., et al., Diesel exhaust particulates upregulate histamine receptor $m R N A$ and increase histamine-induced IL-8 and GM-CSF production in nasal epithelial cells and endothelial cells. Clin Exp Allergy, 1999. 29(1): p. 52-9.

353. Saneyoshi, K., et al., IL-4 and IL-6 production of bone marrow-derived mast cells is enhanced by treatment with environmental pollutants. Int Arch Allergy Immunol, 1997. 114(3): p. 237-45.

354. Devouassoux, G., et al., Chemical constituents of diesel exhaust particles induce IL-4 production and histamine release by human basophils. J Allergy Clin Immunol, 2002. 109(5): p. 847-53.

355. Nilsen, A.M., et al., Reduction of IL-12 440 production in activated monocytes after exposure to diesel exhaust particles. Int Arch Allergy Immunol, 2003. 131(3): p. 201-8.

356. Mamessier, E., et al., Diesel exhaust particles enhance T-cell activation in severe asthmatics. Allergy, 2006. 61(5): p. 581-8.

357. Takenaka, H., et al., Enhanced human IgE production results from exposure to the aromatic hydrocarbons from diesel exhaust: direct effects on B-cell IgE production. J Allergy Clin Immunol, 1995. 95(1 Pt 1): p. 103-15.

358. Ple, C., et al., Polycyclic aromatic hydrocarbons reciprocally regulate IL-22 and IL-17 cytokines in peripheral blood mononuclear cells from both healthy and asthmatic subjects. PLoS One, 2015. 10(4): p. e0122372.

359. Devalia, et al., Differences between Cytokine Release from Bronchial Epithelial Cells of Asthmatic Patients and Non-Asthmatic Subjects: Effect of Exposure to Diesel Exhaust Particles. International Archives of Allergy and Immunology, 1999. 118(2-4): p. 437-439.

360. Fahy, O., et al., Synergistic effect of diesel organic extracts and allergen Der $p 1$ on the release of chemokines by peripheral blood mononuclear cells from allergic subjects: involvement of the map kinase pathway. Am J Respir Cell Mol Biol, 2000. 23(2): p. 247-54.

361. Fahy, O., et al., Diesel exposure favors Th2 cell recruitment by mononuclear cells and alveolar macrophages from allergic patients by differentially regulating macrophage-derived chemokine and IFN-gamma-induced protein-10 production. J Immunol, 2002. 168(11): p. 5912-9.

362. Chang, Y., et al., Diesel exhaust exposure favors TH2 cell recruitment in nonatopic subjects by differentially regulating chemokine production. J Allergy Clin Immunol, 2006. 118(2): p. 354-60.

363. Don Porto Carero, A., et al., Increased HLA-DR expression after exposure of human monocytic cells to air particulates. Clin Exp Allergy, 2002. 32(2): p. 296-300.

364. Takizawa, R., et al., Increased expression of HLA-DR and CD86 in nasal epithelial cells in allergic rhinitics: antigen presentation to $T$ cells and up-regulation by diesel exhaust particles. Clin Exp Allergy, 2007. 37(3): p. 420-33.

365. Chan, R.C., et al., Pro-oxidative diesel exhaust particle chemicals inhibit LPS-induced dendritic cell responses involved in T-helper differentiation. J Allergy Clin Immunol, 2006. 118(2): p. 455-65.

366. Braun, A., et al., Differential impact of diesel particle composition on pro-allergic dendritic cell function. Toxicol Sci, 2010. 113(1): p. 85-94.

367. Ames, B.N., J. McCann, and E. Yamasaki, Methods for detecting carcinogens and mutagens with the salmonella/mammalian-microsome mutagenicity test. Mutation Research/Environmental Mutagenesis and Related Subjects, 1975. 31(6): p. 347-363.

368. Clark, C.R. and C.L. Vigil, Influence of rat lung and liver homogenates on the mutagenicity of diesel exhaust particulate extracts. Toxicology and Applied Pharmacology, 1980. 56(1): p. 110115.

369. Clark, C.R., et al., Mutagenicity of diesel exhaust particle extracts: Influence of car type. Fundamental and Applied Toxicology, 1981. 1(3): p. 260-265.

370. King, L.C., et al., Evaluation of the release of mutagens from diesel particles in the presence of physiological fluids. Environ Mutagen, 1981. 3(2): p. 109-21. 
371. Barfknecht, T.R., et al., Human cell mutagenicity of polycyclic aromatic hydrocarbon components of diesel emissions. Dev Toxicol Environ Sci, 1982. 10: p. 277-94.

372. Bieler, C.A., et al., DNA adduct formation from the mutagenic air pollutant 3-nitrobenzanthrone. Mutation Research/Genetic Toxicology and Environmental Mutagenesis, 1999. 439(2): p. 307311.

373. Shi, X.C., et al., Mutagenicity of diesel exhaust particles from an engine with differing exhaust after treatments. J Toxicol Environ Health A, 2010. 73(19): p. 1314-24.

374. Arimoto, T., et al., Generation of reactive oxygen species and 8-hydroxy-2'-deoxyguanosine formation from diesel exhaust particle components in L1210 cells. Jpn J Pharmacol, 1999. 80(1): p. 49-54.

375. Don Porto Carero, A., et al., Genotoxic effects of carbon black particles, diesel exhaust particles, and urban air particulates and their extracts on a human alveolar epithelial cell line (A549) and a human monocytic cell line (THP-1). Environ Mol Mutagen, 2001. 37(2): p. 155-63.

376. Jardim, M.J., et al., Disruption of microRNA expression in human airway cells by diesel exhaust particles is linked to tumorigenesis-associated pathways. Environ Health Perspect, 2009. 117(11): p. 1745-51.

377. Zhou, F., et al., Effects of diesel exhaust particles on microRNA-21 in human bronchial epithelial cells and potential carcinogenic mechanisms. Mol Med Rep, 2015. 12(2): p. 2329-35.

378. Kaira, K., et al., Prognostic significance of L-type amino acid transporter 1 expression in resectable stage I-III nonsmall cell lung cancer. Br J Cancer, 2008. 98(4): p. 742-748.

379. Kaira, K., et al., Prognostic significance of I-type amino acid transporter 1 (LAT1) and 4F2 heavy chain (CD98) expression in stage I pulmonary adenocarcinoma. Lung Cancer, 2009. 66(1): p. 120126.

380. Le Vee, M., et al., Aryl hydrocarbon receptor-dependent up-regulation of the heterodimeric amino acid transporter LAT1 (SLC7A5)/CD98hC (SLC3A2) by diesel exhaust particle extract in human bronchial epithelial cells. Toxicol Appl Pharmacol, 2016. 290: p. 74-85.

381. Shah, P.P., et al., Ubiquilin1 represses migration and epithelial-to-mesenchymal transition of human non-small cell lung cancer cells. Oncogene, 2015. 34(13): p. 1709-1717.

382. Yadav, S., et al., MIR155 Regulation of Ubiquilin1 and Ubiquilin2: Implications in Cellular Protection and Tumorigenesis. Neoplasia, 2017. 19(4): p. 321-332.

383. Bao, L., et al., Mutagenicity of diesel exhaust particles mediated by cell-particle interaction in mammalian cells. Toxicology, 2007. 229(1-2): p. 91-100.

384. Landvik, N.E., et al., Effects of nitrated-polycyclic aromatic hydrocarbons and diesel exhaust particle extracts on cell signalling related to apoptosis: Possible implications for their mutagenic and carcinogenic effects. Toxicology, 2007. 231(2): p. 159-174.

385. Matsunaga, T., et al., Exposure to 9,10-phenanthrenequinone accelerates malignant progression of lung cancer cells through up-regulation of aldo-keto reductase 1B10. Toxicology and Applied Pharmacology, 2014. 278(2): p. 180-189.

386. Thomas, P., et al., Altered human monocyte/macrophage function after exposure to diesel exhaust particles. Environmental Science and Pollution Research, 1995. 2(2): p. 69-72.

387. Saito, Y., et al., Effects of diesel exhaust on murine alveolar macrophages and a macrophage cell line. Exp Lung Res, 2002. 28(3): p. 201-17.

388. Lundborg, M., et al., Aggregates of ultrafine particles impair phagocytosis of microorganisms by human alveolar macrophages. Environmental Research, 2006. 100(2): p. 197-204.

389. Yin, X.J., et al., Suppression of phagocytic and bactericidal functions of rat alveolar macrophages by the organic component of diesel exhaust particles. Journal of toxicology and environmental health. Part A, 2007. 70(10): p. 820-8. 
390. Sarkar, S., et al., Suppression of the NF-kappaB pathway by diesel exhaust particles impairs human antimycobacterial immunity. J Immunol, 2012. 188(6): p. 2778-93.

391. Müller, L., et al., Diesel exhaust particles modify natural killer cell function and cytokine release. Particle and Fibre Toxicology, 2013. 10: p. 16-16.

392. Jaspers, I., et al., Diesel exhaust enhances influenza virus infections in respiratory epithelial cells. Toxicol Sci, 2005. 85(2): p. 990-1002.

393. Ito, T., et al., Effect of diesel exhaust particles on mRNA expression of viral and bacterial receptors in rat lung epithelial L2 cells. Toxicology Letters, 2006. 165(1): p. 66-70.

394. Ciencewicki, J., et al., Diesel exhaust enhances virus- and poly(I:C)-induced Toll-like receptor 3 expression and signaling in respiratory epithelial cells. Am J Physiol Lung Cell Mol Physiol, 2006. 290(6): p. L1154-63.

395. Nokso-Koivisto, P. and E. Pukkala, Past exposure to asbestos and combustion products and incidence of cancer among Finnish locomotive drivers. Occup Environ Med, 1994. 51(5): p. 330-4.

396. Stiegel, M.A., et al., Inflammatory Cytokines and White Blood Cell Counts Response to Environmental Levels of Diesel Exhaust and Ozone Inhalation Exposures. PLoS One, 2016. 11(4): p. e0152458.

397. Mosbach, M., M. Wiener-Schmuck, and A. Seidel, Influence of coexposure of ozone with quartz, latex, albumin, and LPS on TNF- $\alpha$ and chemotactic factor release by bovine alveolar macrophages in vitro. Inhalation Toxicology, 1996. 8(6): p. 625-638.

398. Lacourt, A., et al., Pleural mesothelioma and occupational coexposure to asbestos, mineral wool, and silica. American Journal of Respiratory and Critical Care Medicine, 2013. 187(9): p. 977-982.

399. Qu, S.-X., et al., DNA adducts in coal miners: association with exposures to diesel engine emissions. Biomarkers, 1997. 2(2): p. 95-102.

400. Hoffmann, B. and K.H. Jockel, Diesel exhaust and coal mine dust: lung cancer risk in occupational settings. Ann N Y Acad Sci, 2006. 1076: p. 253-65.

401. Karagianes, M.T., R.F. Palmer, and R.H. Busch, Effects of inhaled diesel emissions and coal dust in rats. Am Ind Hyg Assoc J, 1981. 42(5): p. 382-91.

402. Castranova, V., et al., The response of rat alveolar macrophages to chronic inhalation of coal dust and/or diesel exhaust. Environ Res, 1985. 36(2): p. 405-419.

403. Lewis, T.R., et al., A chronic inhalation toxicity study of diesel engine emissions and coal dust, alone and combined. Dev Toxicol Environ Sci, 1986. 13: p. 361-80.

404. Beckett, W., et al., Adverse Effects of Crystalline Silica Exposure. Am J Respir Crit Care Med, 1997. 155: p. 8.

405. Yassin, A., F. Yebesi, and R. Tingle, Occupational exposure to crystalline silica dust in the United States, 1988-2003. Environ Health Perspect, 2005. 113(3): p. 255-60.

406. Hnizdo, E., Chronic obstructive pulmonary disease due to occupational exposure to silica dust: a review of epidemiological and pathological evidence. Occup Environ Med, 2003. 60(4): p. 237243.

407. Madl, A.K., et al., State-of-the-science review of the occupational health hazards of crystalline silica in abrasive blasting operations and related requirements for respiratory protection. J Toxicol Environ Health B Crit Rev, 2008. 11(7): p. 548-608.

408. Maciejewska, A., Health effects of occupational exposure to crystalline silica in the light of current research results. Med Pr, 2014. 65(6): p. 799-818.

409. Liao, C.M., et al., Ceramics manufacturing contributes to ambient silica air pollution and burden of lung disease. Environ Sci Pollut Res Int, 2015. 22(19): p. 15067-79.

410. Gauderman, W.J., et al., The effect of air pollution on lung development from 10 to 18 years of age. N Engl J Med, 2004. 351(11): p. 1057-67. 
411. Garshick, E., et al., Lung cancer in railroad workers exposed to diesel exhaust. Environ Health Perspect, 2004. 112(15): p. 1539-43.

412. McCreanor, J., et al., Respiratory effects of exposure to diesel traffic in persons with asthma. $\mathrm{N}$ Engl J Med, 2007. 357(23): p. 2348-58.

413. Lucking, A.J., et al., Particle traps prevent adverse vascular and prothrombotic effects of diesel engine exhaust inhalation in men. Circulation, 2011. 123(16): p. 1721-8.

414. Beatty, T.K. and J.P. Shimshack, School buses, diesel emissions, and respiratory health. J Health Econ, 2011. 30(5): p. 987-99.

415. Kachuri, L., et al., Workplace exposure to diesel and gasoline engine exhausts and the risk of colorectal cancer in Canadian men. Environ Health, 2016. 15(1): p. 4.

416. Phibbs, B.P., R.E. Sundin, and R.S. Mitchell, Silicosis in Wyoming bentonite workers. Am Rev Respir Dis, 1971. 103(1): p. 1-17.

417. Suratt, P.M., et al., Acute silicosis in tombstone sandblasters. Am Rev Respir Dis, 1977. 115(3): p. 521-9.

418. Seaton, A. and J.W. Cherrie, Quartz exposures and severe silicosis: a role for the hilar nodes. Occup Environ Med, 1998. 55(6): p. 383-6.

419. Friedman, G.K., et al., Notes from the field: silicosis in a countertop fabricator - Texas, 2014. MMWR Morb Mortal Wkly Rep, 2015. 64(5): p. 129-30.

420. Halldin, C.N., et al., Debilitating lung disease among surface coal miners with no underground mining tenure. J Occup Environ Med, 2015. 57(1): p. 62-7.

421. Nicol, L.M., et al., Six cases of silicosis: implications for health surveillance of stonemasons. Occup Med (Lond), 2015. 65(3): p. 220-5.

422. Banks, D.E., et al., Silicosis in silica flour workers. Am Rev Respir Dis, 1981. 124(4): p. 445-50.

423. Saiyed, H.N., et al., Silicosis in slate pencil workers: I. An environmental and medical study. Am J Ind Med, 1985. 8(2): p. 127-33.

424. Landrigan, P.J., et al., Silicosis in a grey iron foundry. The persistence of an ancient disease. Scand J Work Environ Health, 1986. 12(1): p. 32-9.

425. Ng, T.P. and S.L. Chan, Lung function in relation to silicosis and silica exposure in granite workers. Eur Respir J, 1992. 5(8): p. 986-91.

426. Love, R.G., et al., Respiratory health effects of opencast coalmining: a cross sectional study of current workers. Occup Environ Med, 1997. 54(6): p. 416-23.

427. Antao, V.C., et al., High prevalence of silicosis among stone carvers in Brazil. Am J Ind Med, 2004. 45(2): p. 194-201.

428. Ferreira, A.S., et al., Progressive massive fibrosis in silica-exposed workers. High-resolution computed tomography findings. J Bras Pneumol, 2006. 32(6): p. 523-8.

429. Ozmen, C.A., et al., MDCT Findings of Denim-Sandblasting-Induced Silicosis: a cross-sectional study. Environmental Health, 2010. 9(1): p. 17.

430. Bakan, N.D., et al., Silicosis in denim sandblasters. Chest, 2011. 140(5): p. 1300-1304.

431. CDC, Pneumoconiosis and advanced occupational lung disease among surface coal miners--16 states, 2010-2011. MMWR Morb Mortal Wkly Rep, 2012. 61(23): p. 431-4.

432. Siribaddana, A.D., et al., A study on silicosis among employees of a silica processing factory in the Central Province of Sri Lanka. Ceylon Med J, 2016. 61(1): p. 6-10.

433. Davis, J.M., J. Ottery, and A. le Roux, The effect of quartz and other non-coal dusts in coalworkers' pneumoconiosis. Part II. Lung autopsy study. Inhaled Part, 1975. 4 Pt 2: p. 691-702.

434. Ahlman, K., A.L. Backman, and T. Partanen, A health survey of granite workers in Finland: radiographic findings, respiratory function, hearing, electric sensory thresholds of the fingers and subjective symptoms. Scand J Work Environ Health, 1975. 1(2): p. 109-16. 
435. Pang, D., S.C. Fu, and G.C. Yang, Relation between exposure to respirable silica dust and silicosis in a tungsten mine in China. Br J Ind Med, 1992. 49(1): p. 38-40.

436. Law, Y.W., et al., Characteristics of workers attending the pneumoconiosis clinic for silicosis assessment in Hong Kong: retrospective study. Hong Kong Med J, 2001. 7(4): p. 343-9.

437. Akgun, M., et al., Silicosis Appears Inevitable Among Former Denim Sandblasters. Chest, 2015. 148(3): p. 647-654.

438. Cohen, R.A., et al., Lung Pathology in U.S. Coal Workers with Rapidly Progressive Pneumoconiosis Implicates Silica and Silicates. Am J Respir Crit Care Med, 2016. 193(6): p. 673-80.

439. Craighead, J.E. and N.V. Vallyathan, Cryptic pulmonary lesions in workers occupationally exposed to dust containing silica. Jama, 1980. 244(17): p. 1939-41.

440. Leigh, J., et al., Quantitative relation between emphysema and lung mineral content in coalworkers. Occup Environ Med, 1994. 51(6): p. 400-7.

441. Dufresne, A., et al., Tentative explanatory variable of lung dust concentration in gold miners exposed to crystalline silica. J Expo Anal Environ Epidemiol, 1998. 8(3): p. 375-98.

442. Schenker, M.B., et al., Pneumoconiosis from agricultural dust exposure among young California farmworkers. Environ Health Perspect, 2009. 117(6): p. 988-94.

443. Nelson, G. and J. Murray, Silicosis at autopsy in platinum mine workers. Occup Med (Lond), 2013. 63(3): p. 196-202.

444. Hayes, R.B., et al., Lung cancer in motor exhaust-related occupations. American Journal of Industrial Medicine, 1989. 16(6): p. 685-695.

445. Steenland, N.K., D.T. Silverman, and R.W. Hornung, Case-control study of lung cancer and truck driving in the Teamsters Union. American Journal of Public Health, 1990. 80(6): p. 670-674.

446. Pfluger, D. and C. Minder, A mortality study of lung cancer among Swiss professional drivers: Accounting for the smoking related fraction by a multivariate approach. Lung Cancer, 1995. 12(3): p. 267.

447. Boffetta, P., et al., Occupational Exposure to Diesel Engine Emissions and Risk of Cancer in Swedish Men and Women. Cancer Causes \& Control, 2001. 12(4): p. 365-374.

448. Laden, F., et al., Historical estimation of diesel exhaust exposure in a cohort study of U.S. railroad workers and lung cancer. Cancer causes \& control : CCC, 2006. 17(7): p. 911-919.

449. Villeneuve, P.J., et al., Occupational exposure to diesel and gasoline emissions and lung cancer in Canadian men. Environ Res, 2011. 111(5): p. 727-35.

450. Koh, D.H., et al., Lung cancer risk in professional drivers in Korea: A population-based proportionate cancer incidence ratio study. J Occup Health, 2015. 57(4): p. 324-30.

451. Peters, S., et al., Estimation of quantitative levels of diesel exhaust exposure and the health impact in the contemporary Australian mining industry. Occupational and Environmental Medicine, 2017. 74(4): p. 282-289.

452. Olsson, A.C., et al., Exposure to Diesel Motor Exhaust and Lung Cancer Risk in a Pooled Analysis from Case-Control Studies in Europe and Canada. American Journal of Respiratory and Critical Care Medicine, 2011. 183(7): p. 941-948.

453. Pintos, J., et al., Occupational exposure to diesel engine emissions and risk of lung cancer: evidence from two case-control studies in Montreal, Canada. Occupational and environmental medicine, 2012. 69(11): p. 787-92.

454. Lipsett, M. and S. Campleman, Occupational exposure to diesel exhaust and lung cancer: a metaanalysis. American Journal of Public Health, 1999. 89(7): p. 1009-1017.

455. Tsoi, C.T. and L.A. Tse, Professional drivers and lung cancer: a systematic review and metaanalysis. Occupational and Environmental Medicine, 2012. 69(11): p. 831-836.

456. Gustafsson, L., et al., Mortality and cancer incidence among Swedish dock workers--a retrospective cohort study. Scand J Work Environ Health, 1986. 12(1): p. 22-6. 
457. Säverin, R., et al., Diesel exhaust and lung cancer mortality in potash mining. American Journal of Industrial Medicine, 1999. 36(4): p. 415-422.

458. Jarvholm, B. and D. Silverman, Lung cancer in heavy equipment operators and truck drivers with diesel exhaust exposure in the construction industry. Occupational and Environmental Medicine, 2003. 60(7): p. 516-520.

459. Garshick, E., et al., Lung Cancer and Vehicle Exhaust in Trucking Industry Workers. Environmental Health Perspectives, 2008. 116(10): p. 1327-1332.

460. API, Oil and Natural Gas Stimulate American Economic and Job Growth. 2014, American Petroleum Institute: online. p. 8.

461. Breitenstein, M., E.J. Esswein, and J. Snawder. NIOSH Field Effort to Assess Chemical Exposures in Oil and Gas Workers: Health Hazards in Hydraulic Fracturing. in NIOSH Intramural Science Conference. 2011. Salt Fork, $\mathrm{OH}$.

462. Diaz-Sanchez, D., et al., Nasal challenge with diesel exhaust particles can induce sensitization to a neoallergen in the human mucosa. J Allergy Clin Immun, 1999. 104(6): p. 1183-1188.

463. IARC. 2012, World Health Organization: International Agency for Research on Cancer: online/lancet.

464. Salvi, S., et al., Acute inflammatory responses in the airways and peripheral blood after shortterm exposure to diesel exhaust in healthy human volunteers. Am J Respir Crit Care Med, 1999. 159(3): p. 702-9.

465. Ghio, A.J., C.B. Smith, and M.C. Madden, Diesel exhaust particles and airway inflammation. Curr Opin Pulm Med, 2012. 18(2): p. 144-50.

466. Ghio, A.J., et al., Controlled human exposures to diesel exhaust. Swiss Med Wkly, 2012. 142: p. w13597.

467. Sagai, M., et al., Biological effects of diesel exhaust particles. I. In vitro production of superoxide and in vivo toxicity in mouse. Free Radic Biol Med, 1993. 14(1): p. 37-47.

468. Ichinose, T., A. Furuyama, and M. Sagai, Biological effects of diesel exhaust particles (DEP). II. Acute toxicity of DEP introduced into lung by intratracheal instillation. Toxicology, 1995. 99(3): $p$. 153-67.

469. Madden, M.C., et al., Effect of ozone on diesel exhaust particle toxicity in rat lung. Toxicol Appl Pharmacol, 2000. 168(2): p. 140-8.

470. Singh, P., et al., Sample characterization of automobile and forklift diesel exhaust particles and comparative pulmonary toxicity in mice. Environ Health Perspect, 2004. 112(8): p. 820-5.

471. Zhao, H., et al., Reactive oxygen species- and nitric oxide-mediated lung inflammation and mitochondrial dysfunction in wild-type and iNOS-deficient mice exposed to diesel exhaust particles. J Toxicol Environ Health A, 2009. 72(8): p. 560-70.

472. Brandt, E.B., et al., Exposure to allergen and diesel exhaust particles potentiates secondary allergen-specific memory responses, promoting asthma susceptibility. J Allergy Clin Immunol, 2015. 136(2): p. 295-303 e7.

473. Kim, B.G., et al., Long-Term Effects of Diesel Exhaust Particles on Airway Inflammation and Remodeling in a Mouse Model. Allergy Asthma Immunol Res, 2016. 8(3): p. 246-56.

474. Sagai, M., A. Furuyama, and T. Ichinose, Biological effects of diesel exhaust particles (DEP). III. Pathogenesis of asthma like symptoms in mice. Free Radic Biol Med, 1996. 21(2): p. 199-209.

475. Bayram, H., et al., The effect of diesel exhaust particles on cell function and release of inflammatory mediators from human bronchial epithelial cells in vitro. Am J Respir Cell Mol Biol, 1998. 18(3): p. 441-8.

476. Ohtoshi, T., et al., Diesel exhaust particles stimulate human airway epithelial cells to produce cytokines relevant to airway inflammation in vitro. J Allergy Clin Immunol, 1998. 101(6 Pt 1): p. 778-85. 
477. Abe, S., et al., Diesel exhaust (DE)-induced cytokine expression in human bronchial epithelial cells: a study with a new cell exposure system to freshly generated DE in vitro. Am J Respir Cell Mol Biol, 2000. 22(3): p. 296-303.

478. Vattanasit, U., et al., Oxidative DNA damage and inflammatory responses in cultured human cells and in humans exposed to traffic-related particles. Int J Hyg Environ Health, 2014. 217(1): p. 23-33.

479. Totlandsdal, A.I., et al., Differential proinflammatory responses induced by diesel exhaust particles with contrasting PAH and metal content. Environ Toxicol, 2015. 30(2): p. 188-96.

480. Steerenberg, P.A., et al., Diesel exhaust particles induced release of interleukin 6 and 8 by (primed) human bronchial epithelial cells (BEAS 2B) in vitro. Exp Lung Res, 1998. 24(1): p. 85-100.

481. Castranova, V., et al., Effect of exposure to diesel exhaust particles on the susceptibility of the lung to infection. Environ Health Perspect, 2001. 109 Suppl 4(Suppl 4): p. 609-12.

482. Siegel, P.D., et al., Effect of diesel exhaust particulate (DEP) on immune responses: contributions of particulate versus organic soluble components. J Toxicol Environ Health A, 2004. 67(3): p. 22131.

483. Mundandhara, S.D., S. Becker, and M.C. Madden, Effects of diesel exhaust particles on human alveolar macrophage ability to secrete inflammatory mediators in response to lipopolysaccharide. Toxicol In Vitro, 2006. 20(5): p. 614-24.

484. Provoost, S., et al., Diesel exhaust particles stimulate adaptive immunity by acting on pulmonary dendritic cells. J Immunol, 2010. 184(1): p. 426-32.

485. Pierdominici, M., et al., Diesel exhaust particle exposure in vitro impacts T lymphocyte phenotype and function. Part Fibre Toxicol, 2014. 11(1): p. 74.

486. Esswein, E.J., et al., Occupational exposures to respirable crystalline silica during hydraulic fracturing. J Occup Environ Hyg, 2013. 10(7): p. 347-56.

487. Vallyathan, V., et al., Freshly fractured quartz inhalation leads to enhanced lung injury and inflammation. Potential role of free radicals. Am J Respir Crit Care Med, 1995. 152(3): p. 1003-9.

488. Sellamuthu, R., et al., Molecular insights into the progression of crystalline silica-induced pulmonary toxicity in rats. J Appl Toxicol, 2013. 33(4): p. 301-12.

489. Satpathy, S.R., et al., Crystalline silica-induced leukotriene B4-dependent inflammation promotes lung tumour growth. Nat Commun, 2015. 6: p. 7064.

490. Vallyathan, V., et al., Enhanced generation of free radicals from phagocytes induced by mineral dusts. Am J Respir Cell Mol Biol, 1992. 6(4): p. 404-13.

491. Shi, X., et al., Generation of Reactive Oxygen Species by Quartz Particles and Its Implication for Cellular Damage. Appl Occup Environl Hyg, 1995. 10(12): p. 1138-1144.

492. Ovrevik, J., et al., $p 38$ and Src-ERK1/2 pathways regulate crystalline silica-induced chemokine release in pulmonary epithelial cells. Toxicol Sci, 2004. 81(2): p. 480-90.

493. Gwinn, M.R., et al., The role of $p 53$ in silica-induced cellular and molecular responses associated with carcinogenesis. J Toxicol Environ Health A, 2009. 72(23): p. 1509-19.

494. Tomaru, M. and M. Matsuoka, The role of mitogen-activated protein kinases in crystalline silicainduced cyclooxygenase-2 expression in A549 human lung epithelial cells. Toxicol Mech Methods, 2011. 21(7): p. 513-9.

495. Sellamuthu, R., et al., Mechanisms of crystalline silica-induced pulmonary toxicity revealed by global gene expression profiling. Inhal Toxicol, 2011. 23(14): p. 927-37.

496. Huaux, F., et al., Role of interleukin-10 in the lung response to silica in mice. Am J Respir Cell Mol Biol, 1998. 18(1): p. 51-9.

497. Pasula, R., et al., Airway delivery of silica increases susceptibility to mycobacterial infection in mice: potential role of repopulating macrophages. J Immunol, 2009. 182(11): p. 7102-9. 
498. Kajiwara, T., et al., Effect of particle size of intratracheally instilled crystalline silica on pulmonary inflammation. J Occup Health, 2007. 49(2): p. 88-94.

499. Creutzenberg, O., et al., Toxicity of a quartz with occluded surfaces in a 90-day intratracheal instillation study in rats. Inhal Toxicol, 2008. 20(11): p. 995-1008.

500. Robertson, S., et al., Diesel exhaust particulate induces pulmonary and systemic inflammation in rats without impairing endothelial function ex vivo or in vivo. Part Fibre Toxicol, 2012. 9: p. 9.

501. Ma, J.Y., et al., Interactive effects of cerium oxide and diesel exhaust nanoparticles on inducing pulmonary fibrosis. Toxicol Appl Pharmacol, 2014. 278(2): p. 135-47.

502. Pronk, A., J. Coble, and P.A. Stewart, Occupational exposure to diesel engine exhaust: a literature review. J Expo Sci Environ Epidemiol, 2009. 19(5): p. 443-57.

503. OshWiki_Contributors Workplace Exposures to Dusts and Aerosols: Diesel Exhaust, 2017.

504. Certificate of Analysis Standard Reference Material 2975 in Diesel Particulate Matter (Industrial Forklift). 2013, US Department of Commerce National Institute of Standards and Technology. p. 11.

505. ICRP, Human respiratory tract model for radiological protection: a report of a task group of the International Commission on Radiological Protection. Ann ICRP, 1994. 24(24): p. 1-482.

506. McDonald, J.D., et al., Characterization of Fine Particle Material in Ambient Air and Personal Samples from an Underground Mine. Aerosol Sci Tech, 2002. 36(11): p. 1033-1044.

507. Antonini, J.M., et al., Introduction of luminol-dependent chemiluminescence as a method to study silica inflammation in the tissue and phagocytic cells of rat lung. Environ Health Perspect, 1994. 102 Suppl 10: p. 37-42.

508. Castranova, V., et al. Pulmonary responses of guinea pigs to consecutive exposures to cotton dust. in Proceedings of the 14th Cotton Dust Research Conference. 1990. Memphis, TN: National Cotton Council

509. Underwood, E., Quantitative Stearology. 1970: Addison-Wesley Publishing Company. 274.

510. Mercer, R.R., M.L. Russell, and J.D. Crapo, Alveolar septal structure in different species. J Appl Physiol (1985), 1994. 77(3): p. 1060-6.

511. Hesterberg, T.W., et al., Non-cancer health effects of diesel exhaust: a critical assessment of recent human and animal toxicological literature. Crit Rev Toxicol, 2009. 39(3): p. 195-227.

512. Brown, B., Hydrofrac Sand in Wisconsin. 2012, University of Wisconsin: University of Wisconsin Extension - Monroe County. p. 1-4.

513. Beckwith, R., Proppants: Where in the World. J Petrol Technol, 2011. 63(04): p. 36-41.

514. Castranova, V. and V. Vallyathan, Silicosis and coal workers' pneumoconiosis. Environ Health Perspect, 2000. 108 Suppl 4(Suppl 4): p. 675-84.

515. Costa, D., et al., A novel type of active site at the surface of crystalline SiO2(a-quartz) and its possible impact on pathogenicity. Can J Chem, 1991. 69(9): p. 1427-1434.

516. Blackford, J.A., Jr., et al., Intratracheal instillation of silica up-regulates inducible nitric oxide synthase gene expression and increases nitric oxide production in alveolar macrophages and neutrophils. Am J Respir Cell Mol Biol, 1994. 11(4): p. 426-31.

517. Wong, O., et al., Mortality among members of a heavy construction equipment operators union with potential exposure to diesel exhaust emissions. Br J Ind Med, 1985. 42(7): p. 435-48.

518. Hart, J.E., E.A. Eisen, and F. Laden, Occupational diesel exhaust exposure as a risk factor for chronic obstructive pulmonary disease. Curr Opin Pulm Med, 2012. 18(2): p. 151-4.

519. Mosser, D.M. and X. Zhang, Activation of Murine Macrophages. Current protocols in immunology / edited by John E. Coligan ... [et al.], 2008. CHAPTER: p. Unit.

520. Joshi, G.N., A.M. Goetjen, and D.A. Knecht, Silica particles cause NADPH oxidase-independent ROS generation and transient phagolysosomal leakage. Mol Biol Cell, 2015. 26(18): p. 3150-64. 
521. Dauber, J.H., et al., Experimental silicosis: morphologic and biochemical abnormalities produced by intratracheal instillation of quartz into guinea pig lungs. Am J Pathol, 1980. 101(3): p. 595612.

522. Heppleston, A.G., Silicotic fibrogenesis: a concept of pulmonary fibrosis. Ann Occup Hyg, 1982. 26(1-4): p. 449-62.

523. Deshazo, R., Current concepts about the pathogenesis of silicosis and asbestosis. J Allergy Clin Immun, 1982. 70(1): p. 41-49.

524. Prieditis, H. and I.Y. Adamson, Alveolar macrophage kinetics and multinucleated giant cell formation after lung injury. J Leukoc Biol, 1996. 59(4): p. 534-8.

525. Co, D.O., et al., $T$ cell contributions to the different phases of granuloma formation. Immunol Lett, 2004. 92(1-2): p. 135-42.

526. Gasse, P., et al., Uric acid is a danger signal activating NALP3 inflammasome in lung injury inflammation and fibrosis. Am J Respir Crit Care Med, 2009. 179(10): p. 903-13.

527. Riteau, N., et al., Extracellular ATP is a danger signal activating P2X7 receptor in lung inflammation and fibrosis. Am J Respir Crit Care Med, 2010. 182(6): p. 774-83.

528. Sager, T.M., et al., Effect of multi-walled carbon nanotube surface modification on bioactivity in the C57BL/6 mouse model. Nanotoxicology, 2014. 8(3): p. 317-27.

529. Sun, B., et al., NADPH Oxidase-Dependent NLRP3 Inflammasome Activation and its Important Role in Lung Fibrosis by Multiwalled Carbon Nanotubes. Small, 2015. 11(17): p. 2087-97.

530. Lee, S., et al., Regulation and Function of the Nucleotide Binding Domain Leucine-Rich RepeatContaining Receptor, Pyrin Domain-Containing-3 Inflammasome in Lung Disease. Am J Respir Cell Mol Biol, 2016. 54(2): p. 151-60.

531. Dostert, C., et al., Innate immune activation through Nalp3 inflammasome sensing of asbestos and silica. Science, 2008. 320(5876): p. 674-7.

532. Peeters, P.M., et al., Silica-induced NLRP3 inflammasome activation in vitro and in rat lungs. Part Fibre Toxicol, 2014. 11: p. 58.

533. Yang, H.M., et al., Effects of diesel exhaust particles (DEP), carbon black, and silica on macrophage responses to lipopolysaccharide: evidence of DEP suppression of macrophage activity. J Toxicol Environ Health A, 1999. 58(5): p. 261-78.

534. Burchiel, S.W., et al., Systemic immunotoxicity in AJ mice following 6-month whole body inhalation exposure to diesel exhaust. Toxicol Appl Pharmacol, 2004. 196(3): p. 337-45.

535. Yin, X.J., et al., Suppression of phagocytic and bactericidal functions of rat alveolar macrophages by the organic component of diesel exhaust particles. J Toxicol Environ Health A, 2007. 70(10): p. 820-8.

536. Dalal, N.S., X.L. Shi, and V. Vallyathan, ESR spin trapping and cytotoxicity investigations of freshly fractured quartz: mechanism of acute silicosis. Free Radic Res Commun, 1990. 9(3-6): p. 259-66.

537. Shoemaker, D.A., et al., Particle activity and in vivo pulmonary response to freshly milled and aged alpha-quartz. Scand J Work Environ Health, 1995. 21 Suppl 2: p. 15-8.

538. Antonini, J.M., et al., Subchronic silica exposure enhances respiratory defense mechanisms and the pulmonary clearance of Listeria monocytogenes in rats. Inhal Toxicol, 2000. 12(11): p. 101736.

539. Farris, B.Y., et al., Pulmonary toxicity following acute coexposures to diesel particulate matter and alpha-quartz crystalline silica in the Sprague-Dawley rat. Inhal Toxicol, 2017. 29(7): p. 322339.

540. Certificate of Analysis Standard Reference Material 2975 in Diesel Particulate Matter (Industrial Forklift). 2013, US Department of Commerce National Institute of Standards and Technology. p. 11.

541. ICRP, in Ann ICRP. 1994. p. 1-482. 
542. Morrow, P.E., Possible mechanisms to explain dust overloading of the lungs. Fundamental and Applied Toxicology, 1988. 10(3): p. 369-384.

543. Morrow, P.E., Dust overloading of the lungs: Update and appraisal. Toxicology and Applied Pharmacology, 1992. 113(1): p. 1-12.

544. Oberdorster, G., Lung Particle Overload: Implications for Occupational Exposures to Particles. Regulatory Toxicology and Pharmacology, 1995. 21(1): p. 123-135.

545. Muhle, H., et al., Dust overloading of lungs: investigations of various materials, species differences, and irreversibility of effects. Journal of Aerosol Medicine, 1990. 1: p. S111-S128.

546. Creutzenberg, O., et al., Clearance and retention of inhaled diesel exhaust particles, carbon black, and titanium dioxide in rats at lung overload conditions. Journal of Aerosol Science, 1990. 21: p. S455-S458.

547. Farris, B.Y., et al., Pulmonary toxicity following acute coexposures to diesel particulate matter and $\alpha$-quartz crystalline silica in the Sprague-Dawley rat. Inhalation Toxicology, 2017. 29(7): $p$. 322-339.

548. Mikolajczyk, U., S. Bujak-Pietrek, and I. Szadkowska-Stanczyk, [Exposure to silica dust in coalmining. Analysis based on measurements made by industrial hygiene laboratories in Poland, 2001-2005]. Med Pr, 2010. 61(3): p. 287-97.

549. Naghizadeh, A., et al., Exposure assessment to dust and free silica for workers of Sangan iron ore mine in Khaf, Iran. Bull Environ Contam Toxicol, 2011. 87(5): p. 531-8.

550. Borm, P.J., L. Tran, and K. Donaldson, The carcinogenic action of crystalline silica: a review of the evidence supporting secondary inflammation-driven genotoxicity as a principal mechanism. Crit Rev Toxicol, 2011. 41(9): p. 756-70.

551. Connellan, S.J., Lung diseases associated with hydrocarbon exposure. Respir Med, 2017. 126: p. 46-51.

552. Valavanidis, A., et al., Pulmonary oxidative stress, inflammation and cancer: respirable particulate matter, fibrous dusts and ozone as major causes of lung carcinogenesis through reactive oxygen species mechanisms. Int J Environ Res Public Health, 2013. 10(9): p. 3886-907.

553. Hamilton, R.F., Jr., S.A. Thakur, and A. Holian, Silica binding and toxicity in alveolar macrophages. Free Radic Biol Med, 2008. 44(7): p. 1246-58.

554. Lawal, A.O., Diesel Exhaust Particles and the Induction of Macrophage Activation and Dysfunction. Inflammation, 2017.

555. Castranova, V., et al., The response of rat alveolar macrophages to chronic inhalation of coal dust and/or diesel exhaust. Environmental Research, 1985. 36(2): p. 405-419.

556. Pierdominici, M., et al., Diesel exhaust particle exposure in vitro impacts T lymphocyte phenotype and function. Particle and Fibre Toxicology, 2014. 11(1): p. 74.

557. Gross, K.B., H.J. White, and K.L. Smiler, Functional and morphologic changes in the lungs after a single intratracheal instillation of silica. Am Rev Respir Dis, 1984. 129(5): p. 833-9.

558. Reasor, M.J. and J.M. Antonini, Pulmonary responses to single versus multiple intratracheal instillations of silica in rats. J Toxicol Environ Health A, 2001. 62(1): p. 9-21.

559. Pollard, K.M., Silica, Silicosis, and Autoimmunity. Front Immunol, 2016. 7: p. 97.

560. Sayan, M. and B.T. Mossman, The NLRP3 inflammasome in pathogenic particle and fibreassociated lung inflammation and diseases. Part Fibre Toxicol, 2016. 13(1): p. 51.

561. Inoue, K. and $\mathrm{H}$. Takano, Biology of diesel exhaust effects on allergic pulmonary inflammation. Yakugaku Zasshi, 2011. 131(3): p. 367-71.

562. Oberdorster, G., J. Ferin, and P.E. Morrow, Volumetric loading of alveolar macrophages (AM): $a$ possible basis for diminished AM-mediated particle clearance. Exp Lung Res, 1992. 18(1): p. 87104. 
563. Morgan, A., et al., The effect of quartz, administered by intratracheal instillation, on the rat lung. I. The cellular response. Environmental Research, 1980. 22(1): p. 1-12.

564. Reiser, K.M., et al., Experimental silicosis. I. Acute effects of intratracheally instilled quartz on collagen metabolism and morphologic characteristics of rat lungs. Am J Pathol, 1982. 107(2): p. 176-85.

565. Sykes, S.E., et al., Dose-dependent effects in the subacute response of the rat lung to quartz. I. The cellular response and the activity of lactate dehydrogenase in the airways. Exp Lung Res, 1983. 5(4): p. 229-43.

566. Halinen, A.J., et al., Diesel particles induce nitric oxide production in murine alveolar macrophages and rat airways. Environ Toxicol Pharmacol, 1999. 7(1): p. 11-8.

567. Takano, H., et al., Diesel exhaust particles enhance lung injury related to bacterial endotoxin through expression of proinflammatory cytokines, chemokines, and intercellular adhesion molecule-1. Am J Respir Crit Care Med, 2002. 165(9): p. 1329-35.

568. Warheit, D.B., T.R. Webb, and K.L. Reed, Pulmonary toxicity screening studies in male rats with M5 respirable fibers and particulates. Inhal Toxicol, 2007. 19(11): p. 951-63.

569. Roursgaard, M., et al., Acute and subchronic airway inflammation after intratracheal instillation of quartz and titanium dioxide agglomerates in mice. ScientificWorldJournal, 2011. 11: p. 80125.

570. Porter, D.W., et al., Time course of pulmonary response of rats to inhalation of crystalline silica: histological results and biochemical indices of damage, lipidosis, and fibrosis. J Environ Pathol Toxicol Oncol, 2001. 20 Suppl 1: p. 1-14.

571. Driscoll, K.E., et al., Pulmonary response to inhaled silica or titanium dioxide. Toxicol Appl Pharmacol, 1991. 111(2): p. 201-10.

572. Lim, H.-B., et al., Involvement of superoxide and nitric oxide on airway inflammation and hyperresponsiveness induced by diesel exhaust particles in mice. Free Radical Biology and Medicine, 1998. 25(6): p. 635-644.

573. Thomas, P., et al., Altered human monocyte/macrophage function after exposure to diesel exhaust particles. Environ Sci Pollut Res Int, 1995. 2(2): p. 69-72.

574. Lundborg, M., et al., Aggregates of ultrafine particles impair phagocytosis of microorganisms by human alveolar macrophages. Environ Res, 2006. 100(2): p. 197-204. 


\section{Acknowledgements}

The authors would like to acknowledge Rosana Schafer of the Department of Microbiology, Immunology, and Cell Biology at West Virginia University School of Medicine for her generous gift of Listeria monocytogenes (strain 10403S, serotype 1).

\section{Disclosure of Interest}

The authors report no conflict of interest.

\section{Disclaimer}

The findings and conclusions in this article are those of the authors and do not necessarily represent the view of the National Institute for Occupational Safety and Health. 


\title{
Curriculum Vitae
}

\section{Breanne Yingling Farris, PhD}

\author{
PERSONAL INFORMATION \\ Department of Immunology, Microbiology and Cell Biology \\ Research in Biomedical Sciences \\ School of Medicine \\ Morgantown, WV
}

EDUCATION

$\mathrm{PhD}$

West Virginia University School of Medicine, Department of Microbiology, Immunology, and Cell Biology, Morgantown, WV.

Bachelor Degrees

Lenoir-Rhyne University, Hickory, NC. BS in Biology with a Concentration in Microbiology, Minor in Chemistry, and Certification in Family Ministry. June 2009.

Magna cum Laude

Honors thesis: Expression of West Nile Virus Non-Structural Protein 2A in E. coli. Advisor: Dr. Catherine H. Schein

High School Diploma $\quad$ Lakeview Christian School, Marion, IN June, 2005

\section{POSITIONS AND APPOINTMENTS}

\author{
January 2012 - Present, CDC/NIOSH Health Effects Laboratory Division, Allergy and \\ Clinical Immunology Branch, Morgantown, WV \\ Regular Fellow \\ Continuing research with Jenny $\mathrm{R}$. Roberts.
}

\begin{abstract}
August 2011 - Present, West Virginia University School of Medicine, Department of Graduate Research in Biomedical Sciences, Immunology and Microbial Pathogenesis Program, Morgantown, WV

Graduate Student in PhD Program

Conducted research in the laboratory of Dr. Jenny R. Roberts at Center for Disease Control National Institute for Occupational Safety and Health (CDC/NIOSH) in the Health Effects and Laboratory Division in the Pathology and Physiology Research Branch. Focused on the exposure of workers to occupational particulates and respiratory health effects resulting from exposure with specific interest in immunological alterations. Studies involve the in vivo exposure of particles associated with the field of hydraulic fracturing and the resulting inflammation, injury, and immunological alterations that occur in the alveolar region of the lung. Analyses include but are not limited to cell differentiation and phenotyping, assessment of cytotoxic effects by
\end{abstract}


colorimetric assay, spectrophotometric assessment of phagocyte oxidant production, histology and morphometry of tissue and flow cytometric analyses.

\section{June 2009 - June 2011, Exela Pharma Sciences, Lenoir, NC}

Quality Control Microbiologist

Conducted quality control experimentation on water for injection, facility and personnel including but not limited to surveillance for microorganisms and endotoxin contamination in a sterile setting for the purpose of developing and manufacturing parenteral pharmaceuticals. Designed and performed research and developmental testing on raw materials, pharmaceutical constituents, and end products manufactured in the facility. Performed method validations for new laboratory experimentation. Developed laboratory protocols and methods to be used in the conduct of quality control operations in the department of microbiology.

\section{June 2008 - August 2008, University of Texas Medical Branch, Department of Biochemistry and Molecular Biology, Galveston, TX \\ Summer Undergraduate Research Fellow}

Designed and carried out experiments to further the investigation of West Nile Viral Nonstructural protein 2A structure and function. Utilized various experimental techniques including but not limited to primer design, DNA sequencing, bacterial transformation and expression of viral protein. Performed research under the dual guidance of Drs. Catherine Schein and Thomas Wood.

\section{August 2006 - May 2009 Lenoir-Rhyne University, Department of Biology and Natural Sciences, Hickory, NC Laboratory and Teaching Assistant}

Carried out duties of preparation of laboratory experiments for basic biology courses as well as microbiology and bacteriology courses. Assisted in the care/husbandry of laboratory specimens/animals. Carried out general lab maintenance and inventory of laboratory supplies/equipment/chemicals. Demonstrated laboratory experiments and instructed students according to assigned curriculum. Proctored exams and assignments while providing general guidance and instruction on technique.

\section{August 2007 - May 2008 Catawba Valley Medical Center, Hickory, NC}

\section{$\underline{\text { Health Careers Intern and Volunteer }}$}

Conducted an internship in various departments of the hospital including the Emergency Department, Speech and Language Pathology Department, Radiology Department, and Wound Healing and Physical Therapy Departments as a part of Health Careers Internship in an observatory capacity. Additionally performed voluntary duties including notation and dictation, instrument/supply inventory/collection and patient companionship.

\section{June 2007 - August 2007 Ball State University, Dept. of Biology, Muncie, IN}

\section{Summer Laboratory Assistant}

Extraction of DNA for determination of spirochete genetic components to determine co-infection rates in Ixodes scapularis under the direction of Fresia Steiner. PI Dr. Robert R. Pinger 


\title{
UNDERGRADUATE STUDENT INSTRUCTION
}

\author{
West Virginia University, School of Medicine
}

- MICB 323 Medical Microbiology Laboratory; Teaching/Laboratory Assistant

\section{Lenoir-Rhyne University, School of Natural Science}

- BIO 105/106 Principles of Biology; Teaching/Laboratory Assistant

- BIO $212 \quad$ Bacteriology; Teaching/Laboratory Assistant

- BIO 245 Plant Anatomy and Physiology; Teaching/Laboratory Assistant

\section{AWARDS AND HONORS}

Best Overall Presentation for 2017 Spring Meeting,

May 2017

A-E SOT Regional Chapter

Graduate Student Travel Support Award,

December 2016

Society of Toxciology

Jennifer Gossling Scholarship,

November 2016

Immunology and Microbial Pathogenesis Program, WVU Foundation

3-Minute Thesis Competition, Finalist

Offices of Graduate Education and Life, WVU

April 2016

3-Minute Thesis Competition, $2^{\text {nd }}$ Place Award for Best Presentation

School of Medicine Preliminaries, WVU

March 2016

Best Oral Presentation by a Graduate Student,

A-E SOT Regional Chapter

May 2014

Graduate Student Travel Assistance

Department of Immunology, Microbiology and Cell Biology, WVU

March 2014

Graduate Student Travel Award,

Office of Graduate Education and Research,

School of Medicine, WVU

March 2014

Graduate Student Travel Award,

A-E SOT Regional Chapter

March 2013

\section{AFFILIATIONS}

American Association for the Advancement of Science, Member August 2016 - Present American Thoracic Society, Trainee Member July 2015 - Present 
Society of Toxicology, Student Member

Allegheny - Erie Society of Toxicology, Student Member

Sigma Xi: The Scientific Research Society, Student Member Galveston Texas Branch

Chi Beta Phi Scientific Society

Lenoir-Rhyne University, Hickory, NC Chapter

Alpha Lambda Delta

Lenoir-Rhyne University, Hickory, NC Chapter

\section{INSTITUTIONAL ORGANIZATIONS}

Graduate Student Organization (GSO)

Outreach and Fundraising Committees Member

Graduate Student Organization (GSO)

Executive Officer and Historian

Graduate Student Organization (GSO)

Executive Officer and Secretary

Graduate Student Organization (GSO)

Outreach Committee Member

\section{PUBLICATIONS (Chronologically)}

\section{Original Published Peer-Reviewed Articles}

1. Roberts JR, Mercer RR, Stefaniak AB, Seehra MS, Geddam UK, Chaudhuri IS, Kyrlidis A, Sager T, Kenyon A, Bilgesu SA, Eye T, Scabilloni JF, Leonard SS, Fix NR, SchweglerBerry D, Farris BY, Wolfarth MG, Porter DW, Castranova V, and Erdely A. Evaluation of Pulmonary and Systemic Toxicity Following Lung Exposure to Graphite Nanoplatelets: a Member of the Graphene Nanomaterial Family. Particle Fibre Toxicol: 2016 June.

2. Shoeb M, Kodali VK., Farris BY, Bishop LM, Meighan T, Salmen R, Eye T, Friend S, Schwegler-Berry D, Roberts JR, Zeidler-Erdely PC, Erdely A, Antonini JM. Oxidative Stress, DNA Methylation, and Telomere Length Changes in Peripheral Blood Mononuclear Cells after Pulmonary Exposure to Metal-Rich Welding Nanoparticles. Nanoimpact. 2017 June.

3. Shoeb M, Kodali VK., Farris BY, Bishop LM, Meighan T, Salmen R, Eye T, Roberts JR, Zeidler-Erdely PC, Erdely A, Antonini JM. Evaluation of the Molecular Mechanisms associated with Cytotoxicity and Inflammation after Pulmonary Exposure to Different Metal Rich Welding Particulates. Nanotoxicology. 2017 June.

4. Farris BY, Antonini JM, Fedan JS, Mercer RR, Roach KA, Chen BT, Schwegler-Berry D, Kashon ML, Barger MW, Roberts JR. 2017. Pulmonary toxicity following acute coexposures to diesel particulate matter and $\alpha$-quartz crystalline silica in the SpragueDawley rat. Inhalation Toxicology. 2017 June. 
5. Farris BY, Roach KA, Fedan JS, Chen BT, Schwegler-Berry D, Mercer RR and Roberts JR. Pulmonary Toxicity following Repeated Co-exposure to Diesel Emission Particulates and $\alpha$-Quartz Crystalline Silica in the Sprague-Dawley Rat. In preparation

6. Farris BY, Barger M, Fedan JS, Chen BT, Friend S, Mercer RR and Roberts JR. Pulmonary Toxicity following Acute Co-Exposures to Crystalline Silica and Diesel

Emission Particulates or Carbon in Rats: differentiating particle-specific versus particle load-effects. In preparation 


\section{PRESENTATIONS (Chronologically)}

\section{Platforms}

1. Yingling, BM, Prusak, DJ, Danecek, P, Mason, PW, Wood, TG and Schein, CH Expression of West Nile Non-Structural Protein 2A in E.coli. North Carolina Academy of Science 2009 Annual Meeting, Warren Wilson College, Asheville, NC (April 4).

2. Yingling, BM, Prusak, DJ, Danecek, P, Mason, PW, Wood, TG and Schein, CH Expression of West Nile Non-Structural Protein 2A in E.coli. Lenoir-Rhyne University, Thesis Competition, May 2009.

3. Yingling BM, McLoughlin C, Antonini JM, MacCuspie RI, Hackley VA, Chen BT, Schwegler-Berry D, and Roberts JR. Alterations in Lung Host Defense After Pulmonary Exposure to Silver Nanoparticles in Rats. Department of Immunology, Microbiology and Cell Biology, West Virginia University, Morgantown, WV. March 7, 2013.

4. Yingling BM, McLoughlin C, Fedan JS, Chen BT, Schwegler-Berry D, and Roberts JR. Intratracheal Co-exposure to Diesel Exhaust Particulate and Crystalline Silica in Rats Potentiates the Inflammatory Effects of Silica in the Lungs. Department of Immunology, Microbiology and Cell Biology, West Virginia University, Morgantown, WV. March 21, 2014.

5. Yingling BM, McLoughlin C, Fedan JS, Chen BT, Schwegler-Berry D, and Roberts JR. Intratracheal Co-exposure to Diesel Exhaust Particulate and Crystalline Silica in Rats Potentiates the Inflammatory Effects of Silica in the Lungs. Allegheny-Erie Regional Chapter, Society of Toxicology Annual meeting, Morgantown, WV, May 15-16, 2014.

6. Farris BY, Fedan, JS, Mercer RR, Chen BT, and Roberts JR. Immunological response to pulmonary co-exposure to Diesel Emission and Silica. Department of Immunology, Microbiology and Cell Biology, West Virginia University, Morgantown, WV. November 20, 2014.

7. Farris BY, Fedan JS, Mercer RR, Chen BT, and Roberts JR. Pulmonary Co-Exposure to Diesel Emission Particulate and Crystalline Silica; An Investigation into Respiratory Inflammation and the Development of Lung Disease. Department of Immunology, Microbiology and Cell Biology, West Virginia University, Morgantown, WV. November, 2015.

8. Farris BY, Fedan JS, Barger MW, and Roberts JR. Immunologic response to pulmonary co-exposure to Diesel Emission and Silica. Three-Minute Thesis Competition, West Virginia University School of Medicine, Morgantown, WV. April , 2016.

9. Farris BY, Fedan JS, Mercer RR, Chen BT, and Roberts JR. Pulmonary Co-Exposure to Diesel Emission Particulate and Crystalline Silica; An Investigation into Respiratory Inflammation and the Development of Lung Disease. Department of Immunology, Microbiology and Cell Biology, West Virginia University, Morgantown, WV. April 7, 2016. 
10. Farris BY, Fedan JS, Barger MW, and Roberts JR. Immunologic response to pulmonary co-exposure to Diesel Emission and Silica. Three-Minute Thesis Finals, West Virginia University, Morgantown, WV. April 7, 2016.

11. Farris BY, Fedan JS, Chen BT, Antonini JM, Schwegler-Berry D, Barger MW and Roberts JR. Co-Exposure to Airborne particulate in Industrial settings: An Investigation into the Pulmonary Toxicity of Diesel Particulate Matter and Crystalline Silica. Department of Immunology, Microbiology and Cell Biology, West Virginia University, Morgantown, WV. May 4, 2017.

12. Farris BY, Fedan JS, Chen BT, Antonini JM, Schwegler-Berry D, Barger MW and Roberts JR. The Effects of Pulmonary Co-Exposure to Diesel Particulate Matter and Crystalline Silica in Rats Using Two Models of Exposure. Allegheny-Erie Regional Chapter, Society of Toxicology Annual meeting, Morgantown, WV, May 17-18, 2017.

\section{Posters}

1. Yingling, BM, Prusak, DJ, Danecek, P, Mason, PW, Wood, TG and Schein, CH Expression of West Nile Non-Structural Protein 2A in E.coli. University of Texas Medical Branch, Summer Undergraduate Research Fellowship Presentations 2008, University of Texas Medical Branch, Galveston, TX.

2. Yingling, BM, Prusak, DJ, Danecek, P, Mason, PW, Wood, TG and Schein, CH Expression of West Nile Non-Structural Protein 2A in E.coli. North Carolina Academy of Science 2009 Annual Meeting, Warren Wilson College, Asheville, NC (April 4).

3. Yingling BM, McLoughlin C, Antonini JM, MacCuspie RI, Hackley VA, Chen BT, Schwegler-Berry D, and Roberts JR. Alterations in Lung Host Defense After Pulmonary Exposure to Silver Nanoparticles in Rats. Van Liere Annual Research Day, West Virginia University, Morgantown, WV. February 28-March 1, 2013.

4. Yingling BM, McLoughlin C, Antonini JM, MacCuspie RI, Hackley VA, Chen BT, Schwegler-Berry D, and Roberts JR. Alterations in Lung Host Defense After Pulmonary Exposure to Silver Nanoparticles in Rats. Society of Toxicology Annual Meeting, San Antonio, TX. March 10-14 ${ }^{\text {th }}, 2013$. Toxicol Sci: The Toxicologist, 132 (1): A1350, p289290, 2013.

5. Yingling BM, McLoughlin C, Antonini JM, MacCuspie RI, Hackley VA, Chen BT, Schwegler-Berry D, and Roberts JR. Alterations in Lung Host Defense After Pulmonary Exposure to Silver Nanoparticles in Rats. Allegheny-Erie Regional Chapter, Society of Toxicology Annual meeting, Morgantown, WV, May 30-31, 2013.

6. Yingling BM, McLoughlin C, Fedan JS, Chen BT, and Roberts JR. Intratracheal Coexposure to Diesel Exhaust Particulate and Crystalline Silica in Rats. Van Liere Annual Research Day, West Virginia University, Morgantown, WV. February 27-28, 2014.

7. Yingling BM, McLoughlin C, Fedan JS, Chen BT, Schwegler-Berry D, and Roberts JR. Intratracheal Co-exposure to Diesel Exhaust Particulate and Crystalline Silica in Rats Potentiates the Inflammatory Effects of Silica in the Lungs. Society of Toxicology Annual Meeting, Phoenix, AZ, March 2014. Toxicol Sci: The Toxicologist, 138 (1): A1242a, p329, 2014. 
8. Roberts JR, Sager T, Bishop L, Mercer RR, Stefaniak AB, Yanamala N, Leonard SS, Roach KA, Schwegler-Berry D, Chaudhuri IS, Kyrlidis A, Farris BY, McLoughlin CE, Eye T, Kodali V, Wolfarth M, Porter DW, Castranova V, and Erdely A. Characterization of lung toxicity following pulmonary exposure to graphene nanoparticles in different oxidized forms. Society of Toxicology Annual Meeting, New Orleans, LA, March 13-17 2016. Toxicol Sci: The Toxicologist, 150 (1): A3496, p586, 2016.

9. Shoeb M, Kodali VK, Erdely A, Meighan TG, Salmen R, Eye T, Zeidler-Erdely PC, Farris BY, Roberts JR, and Antonini JM. Reactive Oxygen Species-Induced DNA Effects of Peripheral Blood Mononuclear Cells Recovered from Rats after Pulmonary Exposure to Welding Fume. Society of Toxicology Annual Meeting, New Orleans, LA, March 13-17 2016. Toxicol Sci: The Toxicologist, 150 (1): A1289, p68, 2016.

10. Fedan JS, Thompson JA, Farris BY, McKinney W, Cumpston AM, Jackson MC, Russ KA, and Roberts JR. Pulmonary Responses Following Inhalation of Sand Dust Collected from Hydraulic Fracturing Operations. Annual Meeting, New Orleans, LA, March 13-17 2016. Toxicol Sci: The Toxicologist, 150 (1): A1284, p66, 2016.

11. Farris BY, Fedan, JS, Mercer RR, Chen BT, and Roberts JR. Repeated Co-exposure to Diesel Exhaust Particulate and Crystalline Silica in Rats. American Thoracic Society International Conference, San Francisco, CA, May 13-18, 2016. Am J Respir Crit Care Med, 193;2016:A5407

12. Shoeb M, Kodali VK, Erdely A, Meighan TG, Salmen R, Eye T, Zeidler-Erdely PC, Farris BY, Roberts JR, Antonini JM. In vivo and in vitro Analysis of Inflammatory Markers and Epigenetic Effects after Welding Fume Treatment. Allegheny-Erie Regional Chapter, Society of Toxicology Annual meeting, Morgantown, WV, May 18-19, 2016.

13. Shoeb M, Kodali VK, Meighan TG, Salmen R, Bishop LM, Eye T, Farris BY, Roberts JR, Zeidler-Erdely PC, Erdely A, Antonini JM. Mechanistic Evaluation of Oxidant Generation and the Development of Inflammation after Pulmonary Exposure to Metal-Rich Welding Nanoparticles. $8^{\text {th }}$ Internation Nanotoxicology Congress. Boston, MA June 1-4, 2016.

14. Farris BY, Barger MW, Fedan JS, Chen BT, Schwegler-Berry D, Friend S, Antonini JM, and Roberts JR. Effects on Pulmonary Host Defense following Acute Exposure to Diesel Emission Particulate and Crystalline Silica in Combination. Society of Toxicology Annual Meeting, Baltimore, MD, March 2017. Toxicol Sci: The Toxicologist, 156 (1): A2378, p324, 2017. 